NOISE CONTROL IN NOVEL POWER ELECTRONICS FOR SMART GRIDS 


\section{Noise Control in Novel Power}

\section{Electronics For SMART Grids}

Niek Moonen 


\section{Members of the graduation committee:}

\section{Chairman \& Secretary:}

Prof. dr. J. N. Kok

\section{Promoter:}

Prof. dr. ir. F. B. J. Leferink

\section{Internal Members:}

Prof. dr. ir. B. Nauta

Prof. dr. J. A. Ferreira

\section{External members:}

Prof. dr. D. W. P. Thomas (University of Nottingham)

Prof. dr. ir. M. J. Bentum (Eindhoven University of Technology)

Dr. ir. A. Roc'h (Eindhoven University of Technology)

Dr. F. Grassi (Polytechnic University of Milan)

Dr. R. Smoleński (University of Zielona Góra)

The research described in this thesis was carried out in the Telecommunication Engineering Group, which is part of the Faculty of Electrical Engineering, Mathematics and Computer Science at the University of Twente, Enschede, the Netherlands. NW' $\begin{aligned} & \text { The author's Ph.D. position was funded by the Netherlands Or- } \\ & \text { ganisation for Scientific Research (NWO) }\end{aligned}$

Copyright (c) 2019 by Niek Moonen

All rights reserved. No part of this publication may be reproduced, stored in a retrieval system, or transmitted, in any form or by any means, electronic, mechanical, photocopying, recording, or otherwise, without the prior written consent of the copyright owner.

ISBN: 978-90-365-4866-3

DOI: $10.3990 / 1.9789036548663$

Printed by Gildeprint

Typeset in $\mathrm{ATEX}_{\mathrm{E}} 2_{\varepsilon}$ 


\title{
Noise Control in Novel Power Electronics FOR SMART GRID
}

\author{
DisSERTATION
}

to obtain

the degree of doctor at the University of Twente, on the authority of the rector magnificus, Prof. dr. T. T. M. Palstra

on account of the decision of the graduation committee, to be publicly defended

on Friday $11^{\text {th }}$ October 2019 at 16:45h

by

Dominicus Johannes Guilielmus Moonen

born on $6^{\text {th }}$ May 1990

in Veghel, The Netherlands 
This dissertation has been approved by:

The Promotor:

Prof. dr. ir. F. B. J. Leferink

536329-L-bw-Moonen

Processed on: 3-10-2019

PDF page: 4 


\section{Summary}

As society and technology are developing, the amount of electrically powered devices is ever increasing. The traditional electrical grid, structured in a hierarchical way, is not capable of sustaining the rapid development and implementation of a more dynamical consumer. By incorporating solar panels and wind turbines at farm, businesses and even households the consumer becomes a producer as well. This transforms the conventional grid into a more dynamical and also distributed one. The project of which this thesis was a part of, deals with the integration of renewable energy by applying a new architecture that enables point-to-point power transmission and thus reduces instabilities and improves dynamical behavior. The key objective for the University of Twente (UT) is to find the best options for reducing interference, associated with fast switching semiconductors as applied in the novel converter type.

This thesis starts with the development of a theoretical model based on the control signals applied to switching power devices. The model can be used to predict and estimate the conducted Electroagnetic Interference (EMI) generated in switching devices using sinusoidal Pulse Width Modulation (sPWM) waveforms. The model was verified in a conducted emission measurement. Combining the model with a radiated emission estimation model, gives a description of a noise source that is applied in the newly developed multi-level converter.

For application in multi-level converters a sPWM generator was developed, which with its flexibility is used to perform measurements on Galium-Nitride $(\mathrm{GaN})$ and Silicon-Carbide (SiC) based DC/AC converters, and multilevel converters. In case of conducted EMI measurements, the results were used to verify the theoretical model. In case of the magnetic radiated EMI measurements, a time domain measurement technique was developed that is comparable to using a traditional EMI receiver. The technique reduces measurement times from minutes to several seconds per orientation and placement. In case of large stacked multi-level converters it was deemed necessary to asses the magnetic radiation produced in such a structure. The electric field measurements are eventually used together with the developed mathematical model to determine the effective radiation efficiency of the system under test.

The developed technique of determining the effective radiation efficiency, together with the concept for optimal placement of EMI in fully integrated 
systems are considered to be part of the main contributions of this thesis and can be seen as Mitigation through EMI placement. Another mitigation technique, which is a fairly classical Electromagnetic Compatibility (EMC) one, consist of implementing a filter along the propagation path of the disturbance. Part of the research objectives was developing behavioral circuit models based on full wave model and thus optimized filters implementing nano-crystalline materials

This thesis contributes with the development of an automated method for back annotating field effects into equivalent circuit simulations. Part of this work was the development of Gauss-Newton optimization algorithm that can fit impedance curves to equivalent circuit elements. This was applied to measurements and full-wave 3D simulations of relatively simple components like capacitors, which showed optimal capacitor placement can be investigated through circuit simulations rather then Electro-Magnetic (EM) field simulations. Extending the research to more complex structures and components, required the development of a 3D full wave high frequency models. This has been done for a two phased sectionally winded Common Mode Choke (CMC), incorporating complex permeabilities through a dispersion model.

Overall it can be concluded that this thesis has contributed to the development of the future electrical grid, by investigating components that are attributed to the Multifunctional Multilevel Modular Converter (M3C), which is considered to enable the development of the 'smart grid'. Much work still needs to be done, from refining and applying the developed measurement techniques to larger and fully integrated systems to developing more accurate and faster fitting algorithms for determining equivalent circuit component values. Also the effect of proposed mitigation techniques on various functionalities of the M3C have to be investigated. 


\section{Samenvatting}

Met de ontwikkeling van de maatschappij en de technologie die hiermee gepaard gaat, neemt het aantal elektrisch aangedreven apparaten toe. Het traditionele elektriciteitsnetwerk is gestructureerd in een hiërarchische vorm en is niet in staat de ontwikkeling en implementatie van de dynamische consument te ondersteunen. Door het toevoegen van zonnepanelen en windturbines aan boerderijen, bedrijven en alledaagse huishoudens vindt er een transitie plaats van consument naar producent. Dit leidt uiteindelijk tot de transformatie naar een meer dynamisch en gedistribueerd netwerk. Het project waar deze thesis onderdeel vanuit maakt, houdt zich bezig met de integratie van nieuwe "renewable" energie voorzieningen door middel van het toepassen van een nieuw ontwikkelde vermogenselektronica architectuur. Deze architectuur is in staat om vermogen onderling te leveren van en naar gebruikers en de daarbij mogelijke instabiliteit te reduceren. Het hoofddoel voor de universiteit Twente, en dus deze thesis, is het vinden van de beste mogelijkheden om storingen te verminderen die voortkomen uit de snel schakelende vermogenselektronica zoals gebruikt in de nieuw ontwikkelde type omvormer.

Deze thesis start met de ontwikkeling van een theoretisch model dat is afgeleid van de signalen die toegepast worden op de schakelende componenten. Het model kan gebruikt worden om te voorspellen welke elektromagnetische interferentie ontstaat in schakelende voedingen die aangedreven worden door sinusvormige puls modulatie golfvormen (sPWM). Het model is geverifieerd door middel van elektrische geleidingsmetingen. Door dit model te combineren met een elektromagnetisch stralingsmodel kan een volledige beschrijving van de storingsbron worden gemaakt die veelvuldig aanwezig is in de nieuw ontwikkelde "multi-level" omvormer.

Doordat er onderzoek gedaan wordt naar specifieke "multi-level" omvormers is er een sPWM generator ontwikkeld die zeer flexibel in te stellen is en gebruiksvriendelijk is. Uiteindelijk is deze gebruikt als aansturing van DC/AC omvormers en "multi-level" omvormers, die gebruikmaken van Gallium-Nitride en Silicium-Carbide halfgeleiders. In het geval van elektromagnetische geleidingsmetingen, zijn de resultaten ter verificatie gebruik voor het eerder ontwikkelde theoretische model. In het geval van magnetische staling, zijn er tijd-domein metingen ontwikkeld en uitgevoerd die één op één te vergelijken zijn met de conventionele meetontvanger. Deze techniek reduceert meettijd 
van enkele minuten naar luttele seconden per oriëntatie en locatie. In het geval van de "multi-level" omvormer, welke een groot geometrisch oppervlak heeft, was het nodig het magnetisch veld op verscheidende locaties te evalueren. Door middel van de nieuwe test techniek is de totale meettijd hierdoor significant afgenomen. De elektrische veld metingen zijn uiteindelijk samen met het ontwikkelde wiskundige model gebruikt om de effectiviteit van de straling te bepalen. Met andere woorden, hoe goed straalt het systeem elektromagnetische straling onder de werkende condities.

De ontwikkelde techniek voor het bepalen van de stralingseffectiviteit, samen met het optimaal "plaatsen" van storingscomponenten in volledig geïntegreerde systemen worden gezien als hoofdonderdelen van de bijdrage van deze thesis aan de wetenschap. In een uitspraak samengevat "Mitigation through EMI placement", oftewel "vermindering door middel van EM storingsplaatsing". Andere, meer conventionele, methodes bestaan uit het invoegen van filters tussen de storingsbron en het slachtoffer. Deel van het hier uitgevoerde onderzoek was het ontwikkelen van circuit modellen gebaseerd op 3D simulaties ter optimalisatie van filters die nano-kristallijne materialen bevatten. Deze thesis heeft een bijdrage geleverd aan een volledig geautomatiseerde methode voor het invoegen van veld-effecten in circuit simulatoren. Onderdeel hiervan was de implementatie van een Gauss-Newton optimalisatie algoritme dat gebruikt kan worden om equivalente circuit component waardes te vinden aan de hand van een ingegeven impedantie curve. Deze techniek is toegepast op zowel de resultaten van metingen als $3 \mathrm{D}$ simulaties voor relatief eenvoudige passieve componenten, zoals condensatoren. Uiteindelijk is ook aangetoond dat de optimale plaatsing van een condensator op deze manier bepaald kan worden, zonder een groot aantal volledige veld simulaties uit te voeren. Uitbreiding naar meer complexe structuren en componenten is mogelijk, maar hiervoor moeten 3D modellen ontwikkeld worden die bruikbaar zijn buiten de normale bandbreedte van operatie. In deze thesis is dit gedaan voor een twee fase gekoppelde smoorspoel (CMC).

In het algemeen kan geconcludeerd worden dat deze thesis bijgedragen heeft aan de ontwikkeling van het toekomstige elektriciteitsnetwerk, door het onderzoeken van componenten die toebehoren aan "Multi-functional Multilevel Modular Converters (M3C)". Dit type omvormer acht men instaat de ontwikkeling van de toekomstige "Smart Grid" te bewerkstelligen. Er moet nog veel uiteenlopend werk verzet worden, van het ontwikkelen van meettechnieken voor grotere en volledig geïntegreerde systemen tot aan snellere optimalisatie algoritmes voor equivalente circuit componentwaarde bepalingen. Ook moet nog bepaald worden of de voorgestelde storingsreductietechnieken een negatief effect hebben op de functionele gedragingen van de omvormers. 


\section{Contents}

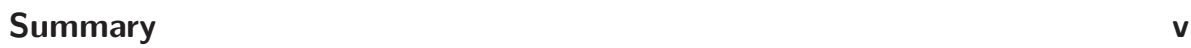

Samenvatting vii

Abbreviations xii

1 Introduction 1

1.1 The Electrical Grid . . . . . . . . . . . . . . . . . . . . 1

1.2 Research Project - Smart Grids . . . . . . . . . . . . . . . 3

1.3 Motivation ...................... . . 4

1.4 Research objectives . . . . . . . . . . . . . . . . . . 5

1.5 Outline of the thesis . . . . . . . . . . . . . 7

2 Noise source modeling 9

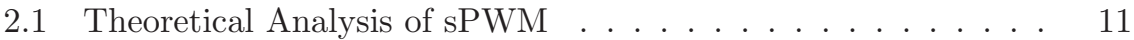

2.1.1 Amplitude. . . . . . . . . . . . . . . . . . 12

2.1 .2 Bandwidth . . . . . . . . . . . . . . . . 13

2.1 .3 Model . . . . . . . . . . . . . . . . . . . . . . . . . . . . 14

2.2 Conducted Emissions . . . . . . . . . . . . . . . . . 15

2.2 .1 Conducted Emission Results . . . . . . . . . . . . 16

2.3 Radiated Emissions . . . . . . . . . . . . . . . . . . . . . . . . . . . 21

2.3.1 Radiated - Electrical Field . . . . . . . . . . . . . . . 21

2.3.2 Electric Field Results . . . . . . . . . . . . . . . . . 21

2.3.3 Radiated - Magnetic Field Strength _. . . . . . . . 27

2.3.4 Magnetic Field Strength Results . . . . . . . . . . . 28

2.4 Summary . . . . . . . . . . . . . . . . . . . . . 29

3 sPWM in M3C 31

3.1 sPWM Generator Design . . . . . . . . . . . . . . . 32

3.1 .1 sPWM Generator . . . . . . . . . . . . . . . . 33

3.1 .2 Implementation . . . . . . . . . . . . . . . . . . . . . . . . . . . . . . .

3.1 .3 Evaluation . . . . . . . . . . . . . . . . 43

3.2 Harmonic Cancellation . . . . . . . . . . . . . . . . . . . . 51

3.2.1 Demonstrator M3C . . . . . . . . . . . . . . . . 52 
3.2.2 Power Shifter Module . . . . . . . . . . . . . . 53

3.2.3 Spectrum of PWM . . . . . . . . . . . . . 55

3.2.4 Numerical Analysis Summary . . . . . . . . . . . . 62

3.2.5 Harmonic Cancellation in Multi SM converter . . . . . . 62

3.2.6 M2C-DC/DC Demonstrator . . . . . . . . . . 65

3.2 .7 Results ..................... 66

3.3 Summary ........................ 68

4 Filtering $\quad 69$

4.1 Automated Equivalent Circuit Values . . . . . . . . . . . . . . 70

4.1.1 Optimization problem ............... 71

4.1.2 Components ................. . . 73

4.1.3 Impedance Fitting . . . . . . . . . . . . . . . . . . . . 77

4.1 .4 Filter . . . . . . . . . . . . . . . . . 78

4.2 CMC - Equivalent Circuit Modeling . . . . . . . . . . . . . . 81

4.2.1 Modeling through Curve Fitting . . . . . . . . . . . 83

4.2.2 Towards a Physics Based Approach . . . . . . . . . . 85

4.2.3 Mutual Coupling Effect . . . . . . . . . . . . 89

4.3 CMC - 3D Full Wave High Frequency Modeling . . . . . . . . . 90

4.3.1 Modeling 3D: FEM . . . . . . . . . . . . . . . 93

4.3 .2 Impedances Extraction . . . . . . . . . . . . . . . . 97

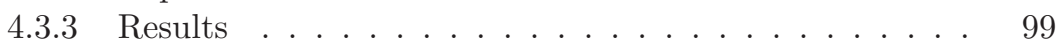

4.4 Layout Optimization . . . . . . . . . . . . . . . 113

4.4 .1 Case Study . . . . . . . . . . . . . . . . . . . . . . . . . 113

4.4 .2 Simulations . . . . . . . . . . . . . . 116

4.4.3 Filter Measurements . . . . . . . . . . . . . . . 121

4.4 Results ..................... 122

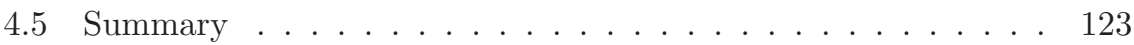

5 EMI Mitigation through EMI Placement $\quad \mathbf{1 2 5}$

5.1 Conducted EMI Mitigation - Optimal Filter Usage . . . . . . . 126

5.1.1 Carrier Frequency Shift . . . . . . . . . . . . . . 126

5.1 .2 Application .................... . . 129

5.1 .3 The Test Setup . . . . . . . . . . . . . . . . 132

5.1 .4 Results .................... 133

5.2 Radiated EMI Mitigation - Optimal System Usage . . . . . . . 134

5.2.1 Concept of Radiation Efficiency . . . . . . . . . . . . . 134

5.2.2 Application - DC/AC Converter . . . . . . . . 136

5.3 Radiation Efficiency Determination . . . . . . . . . . . . . 138

5.4 Summary . . . . . . . . . . . . . . . . . . 142

6 Conclusions and Directions for Further Research 143

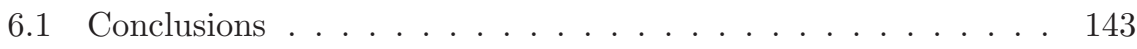

6.1.1 Chapter 2...................... 143

6.1 .2 Chapter 3 . . . . . . . . . . . . . . . 144

6.1.3 Chapter 4..................... 145 


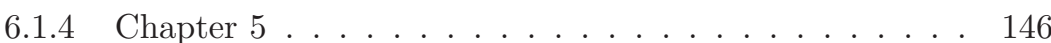

6.2 Directions for Further Research . . . . . . . . . . . . . . . 146

$\begin{array}{llr}7 & \text { Acknowledgments } & 149\end{array}$

8 Biography 153

$\begin{array}{ll}\text { References } & 154\end{array}$

$\begin{array}{lc}\text { List of my Publications } & 164\end{array}$

Appendix

$\begin{array}{lr}\text { A Protection Circuit } & 169\end{array}$

$\begin{array}{lr}\text { B Impedance Measurement ENA } & 171\end{array}$

B.1 E5061B .............................. 171

C Processing Radiation $\quad \mathbf{1 7 5}$

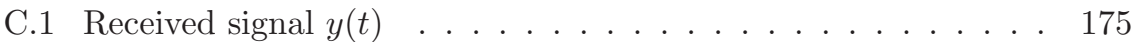

C.2 Supplied signal $x(t) \quad \ldots \ldots \ldots$. . . . . . . . . . 176

C.3 Magnetic field . . . . . . . . . . . . . . . . . . . 177

C.3.1 RE101 . . . . . . . . . . . . . . . 177

C.3.2 Time domain . . . . . . . . . . . . 177

$\begin{array}{lr}\text { D Heater as load } & 179\end{array}$

E Permeability 181

E.1 Data sheet. . . . . . . . . . . . . . . . . . . 181

E.1.1 Direct . . . . . . . . . . . . . . . 182

E.1.2 Indirect . . . . . . . . . . . . . . . . . . 182

E.2 Core measurement . . . . . . . . . . . . . . . . . 182

E.2.1 Adapter usage . . . . . . . . . . . . . . . . . . 182

E.3 Inductor measurement . . . . . . . . . . . . . . . . . . . . . 183

E.4 Comparison of methods . . . . . . . . . . . . . . . . . 184

F Conducted Emission Monitoring $\quad 187$

F.1 The Box . . . . . . . . . . . . . . . . . . . . 187

F.2 Measurement . . . . . . . . . . . . . . . . . . 188

F.3 Usage . . . . . . . . . . . . . . . . . . . . . . . . . . . . . . . 189 


\section{Acronyms}

ADC Analog-to-Digital Converter.

CM Common Mode.

CMC Common Mode Choke.

COTS Commercial Of The Shelf.

DAC Digital-to-Analog Converter.

DM Differential Mode.

DSP Digital Signal Processor.

DSP Digital Signal Processing.

DUT Device Under Test.

EMC Electromagnetic Compatibility.

EMI Electroagnetic Interference.

ENA Enhanced Network Analyzer.

EPC Equivalent Parallel Capacitor.

EPR Equivalent Parallel Resistor.

ERP Effective Radiated Power.

ESL Equivalent Series Inductor.

ESR Equivalent Series Resistor.

FEM Finite Element Method.

FFT Fast Fourier Transform.

FM Frequency Modulation. 
FPGA Field-Programmable Gate Array.

GaN Galium-Nitride.

HDL Hardware Description Language.

HVDC High Voltage Direct Current.

LTI Linear Time-Invariant.

LUT Look-Up Table.

M2C Multilevel Modular Converter.

M3C Multifunctional Multilevel Modular Converter.

MC Multilevel Converters.

MF Multiple Frequencies.

PCB Printed Circuit Board.

PEC Perfect Electric Conductor.

PEEC Partial Equivalent Element Circuit.

PMOD Peripheral Modules.

PS-sPWM Phase Shifted sinusoidal Pulse Width Modulation.

PWM Pulse Width Modulation.

RCM Resistive Coupled Mode.

SHE Selective Harmonic Elimination.

$\mathrm{SiC}$ Silicon-Carbide.

SM Sub-Module.

SMPS Switch Mode Power Supply.

sPWM sinusoidal Pulse Width Modulation.

TDEMI Time-Domain Electromagnetic Interference.

VCVS Voltage Controlled Voltage Source.

VHDL VHSIC Hardware Description Language.

VNA Vector Network Analyzer. 


\section{Chapter 1}

\section{Introduction}

\subsection{The Electrical Grid}

As society and technology are developing, the amount of electrically powered devices per household is ever increasing. As energy in the form of electricity is inherently clean and can be relatively efficiently transformed in any other form of energy (i.e. heat, mechanical, lighting etc.), it is at the center of developing a sustainable society. One could have noticed this transition into a more electrically powered society with the replacement of well established technologies. Considering a common household: the gas stove is being replaced by one based on induction or thermal conduction, central heating boilers based on gas are being replaced by heat pumps, and combustion based vehicles are being replaced by electric vehicles. Next to this, in the Netherlands, cyclists are now using electrically powered bikes. All of this contributes to an increase in the worlds total energy consumption, which can be seen in Figure 1.1. An increase in demand also requires an increase in generation. It is increasing over the past few decades, as can be seen, together with its total waste.

Distributed energy generation is being implemented to cope with rapidly increasing demand of energy. The traditional electrical grid, structured in a hierarchical way, is not capable in its current form of sustaining the electrification of society. By incorporating solar panels and wind turbines at farms, businesses and even households the consumer has become a producer as well. The transformation of the conventional grid into a more dynamical and also distributed one posses several challenges. A ( $\mathrm{r}$ )evolution is required to be able to solve them. Many systems and architectures are proposed, developed and researched to provide/support the next big development in power electronics which many have been calling the Smart Grid. The Smart Grid has regularly come to be described as:

"The next-generation electrical power system that is typified by the increased use of communications and information technology in the generation, delivery and consumption of electrical energy." 


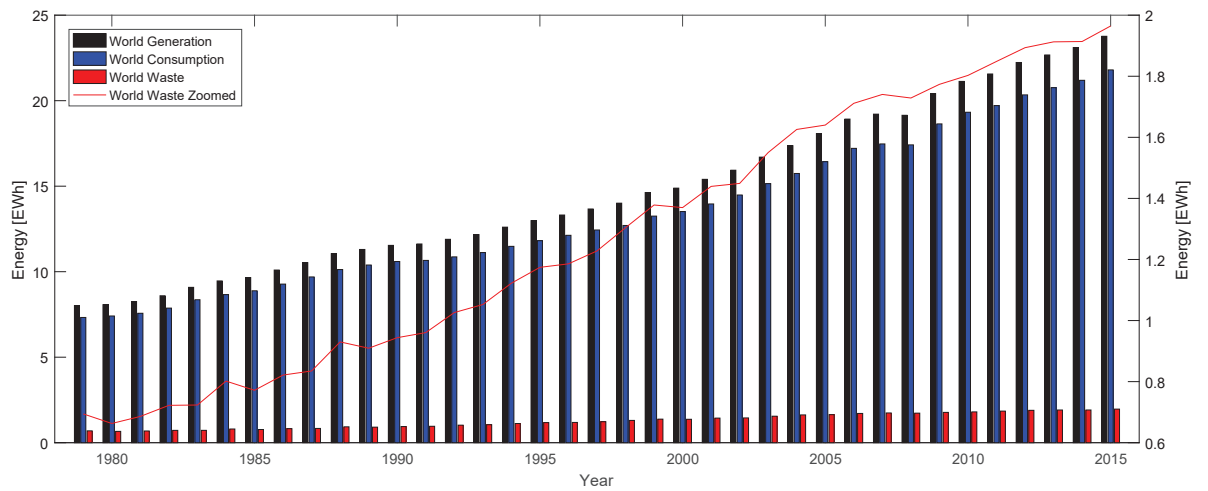

Figure 1.1: World energy generation, consumption and waste. Data acquired from 'EIA - Electricity Data' [1].

Table 1.1, which is adopted from [2], shows a comparison of the envisioned grid compared to existing grid, which depicts several key transformations that either already are being implemented or are under development. Figure 1.2 visualizes the transformation as envisioned. To meet the demand for increased efficiency, flexibility and system capability a novel converter-based solution was proposed and described in [3] called the Multifunctional Multilevel Modular Converter (M3C). Research on the M3C topology is part of a joint project between India and the Netherlands dubbed 'Smart Grids' and funded by The Netherlands Organization for Scientific Research (NWO).

Table 1.1: The smart grid compared with the existing grid adopted from [2].

\begin{tabular}{|c||c|}
\hline \multicolumn{1}{|c||}{ Existing Grid } & Smart Grid \\
\hline Electromechanical & Digital \\
\hline One-Way Communication & Two-Way Communication \\
\hline Centralized Generation & Distributed Generation \\
\hline Hierarchical & Network \\
\hline Few Sensors & Sensors Throughout \\
\hline Blind & Self-Monitoring \\
\hline Manual Restoration & Self-Healing \\
\hline Failures and Blackouts & Adaptive and Islanding \\
\hline Manual Check/Test & Remote Check/Test \\
\hline Limited Control & Pervasive Control \\
\hline Few Customer Choices & Many Customer Choices \\
\hline
\end{tabular}




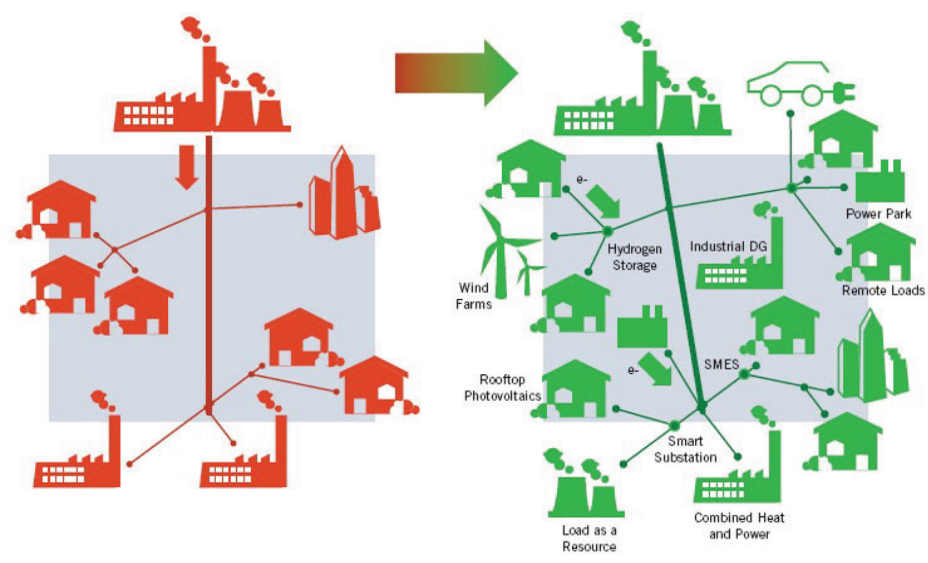

Figure 1.2: The IEEE's version of the Smart Grid involves distributed generation, information networks, and system coordination, a drastic change from the existing utility configurations. Adopted from IEC-IEEE challenge: "100\% Green Computing At The Wrong Location?" [4].

\subsection{Research Project - Smart Grids}

With the development of the future smart grid, more is required from the capabilities of power converters. New methods to perform energy transactions, self-healing and increased resilience to various anomalies exited by electrical networks are the requirements posed on the next generation electrical grid [2]. This research project is aimed at developing solutions by utilizing additional frequencies in Multilevel Modular Converter (M2C) topologies, as was proposed in $[5,6]$ resulting in the development of $\mathrm{M} 3 \mathrm{C}$ systems, i.e. it enables the possibility of power transfer at other frequencies than the conventional DC or $50 \mathrm{~Hz}$.

The idea of utilizing Multiple Frequencies (MF) in a single system is not novel, however its implementation in power transmission is. A main issue is that the powers at different frequencies do not interact 'by nature i.e. by themselves', but only via control of switching power devices which brings an inherent improvement of stability. In the "SMART GRID" project research topics are split between 4 universities (Figure 1.3).

- Technical Univeristy of Delft (TUD)

- University of Twente (UT)

- Indian Institute on Technology, Delhi (IITD)

- Indian Institute on Technology, Bombay (IITB)

Concerning applications in power system the Delft group is mainly focused on hardware research and development and prototyping of devices for power 
systems whereas the IITD and IITB are more interested in the dynamical aspects at system level. Several challenges were identified during the development, most notable are optimal control strategy [7-9], potential Electroagnetic Interference (EMI) issues, and module interactions [3]. The research presented in this thesis is from the UT and it addresses the potential EMI issues that can arise from implementing MF in a system designed with modular fast switching building blocks. The research of UT, described in this thesis, complements the research of TUD, IITD, and IITB as it addresses EMI at the module level.

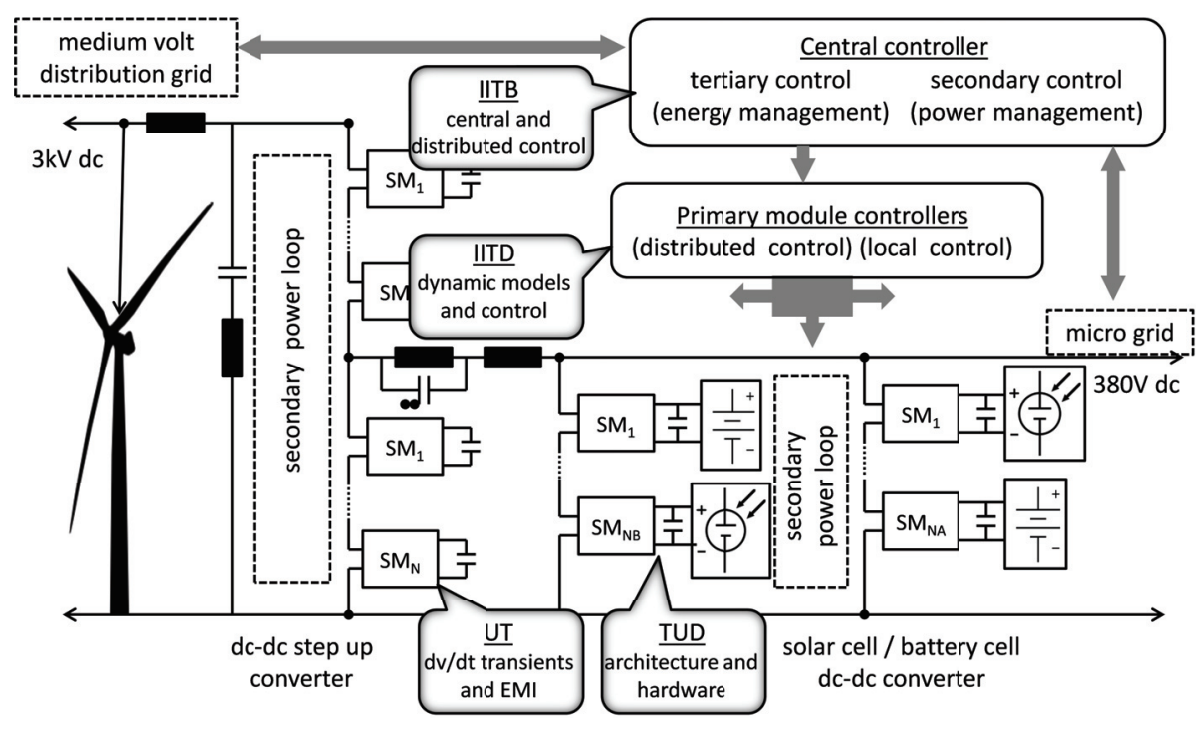

Figure 1.3: Full DC micro-grid and medium voltage demonstrator system.

\subsection{Motivation}

Key features of the M3C system are modularity, multi-functionality and multilevel implementation. The modules that will be used in the system are not going to represent a single, universal converter. Instead, they are intended to be configurable to fit the requirements of a specific device, which implies an increased flexibility of the system. Multi-functionality (or multi-frequency) allows power flow between any source and load in the system, without affecting the other loads and sources. It thus features a controllable network that is capable of operating with multiple-frequencies and is highly dynamic.

The possibility of designing of the novel electrical power system based on a bottom-up approach is described in [3], where it shows that it utilizes power transfer via multiple, different frequencies, using the concept of orthogonal power flow in nestled secondary power loops, as described in $[5,6]$. The principle of orthogonal power flow can be described by using the Fourier series 
expansion to express the non-sinusoidal voltage and current inside a system. The active power is then defined as the mean value of the product of the instantaneous values, in which case all the cross product terms of different (integer valued) frequencies are zero for non-sinusoidal periodic waveforms. As presented in [10], a Modular Multilevel DC converter (M2DC) prototype was developed, which demonstrates some of the key features of the M3C topology. The prototype shows bidirectional DC/DC conversion via two modes of operation, while capacitor charge balancing is achieved using $\mathrm{AC}$ currents. The orthogonal power flow decouples the active power in the different frequencies. By implementing nestled secondary power loops in a $\mathrm{M} 2 \mathrm{C},[6]$ demonstrates that it is possible to add multiple sources without requiring a transformer. Each Sub-Module (SM) allows for a connection of source or load, therefore by increasing the amount of SMs in the system additional sources and loads can be supported.

As part of the TUD objectives a demonstrator was developed, which is described in [10] and can be seen in Figure 1.4. This is considered to be the starting point of the analysis performed in this thesis. The topology is novel and thus not very well known, especially not in the world of Electromagnetic Compatibility (EMC). In this introduction a simplified description is given for its functional behavior, as it is required to understand experiments and analyses performed in this thesis.

The circuit model shown in Figure 1.4 depicts the situation in which the system operates as a DC/DC boost converter, in which a "low" voltage source is applied at the left hand side and the "high" voltage load on the right hand side. The source is decoupled from the system's secondary power loop via a bandstop notch filter consisting of parallel connected inductor $\left(L_{b s}\right)$ and capacitor $\left(C_{b s}\right)$, while the load resistor is disconnected via a low pass filter consisting of a single inductor $\left(L_{o}\right)$. The secondary power loop is then created using the bandpass notch filter consisting of a series connected inductor $\left(L_{b p}\right)$ and capacitor $\left(C_{b p}\right)$. Effectively this results that ideally only the DC-current escapes and enters the secondary power loop, where both the DC-current and ACcurrent are existing. The SM can have different designs, however to reduce the amount of switches and thus reducing complexity of control signal generation it is implemented using a half bridge consisting of two switches. The ACcurrent running in the shown power loop has the effect of balancing the charge between the multiple SMs. Thus it keeps the capacitors charged at all times during operation. From [10] it can be seen that when operating in the resistive coupled mode, the system acts as a voltage controlled voltage source. Based on the set control parameters, which are elaborated on in Chapter 3, the transfer voltage ratio can be controlled.

\subsection{Research objectives}

As the research objectives of this thesis are related to the EMI generated, it is imperative to first simplify and model sub-systems. It was shown that 


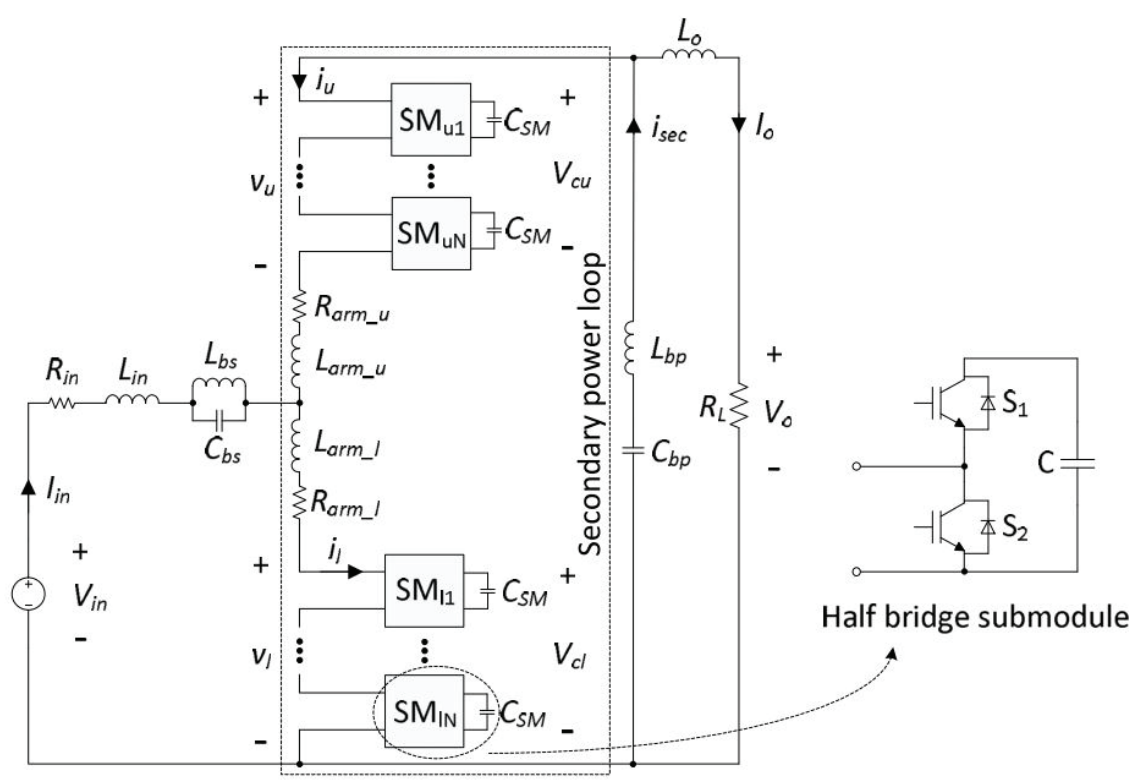

Figure 1.4: Circuit model of the tuned filter M3C, adopted from [10].

investigation in the generated EMI remains difficult, due to the complexity and flexibility of the total system and the dependency on (often unpredictable) parasitics that only surface during integration stage of the total system [11-14]. Throughout this thesis the complexity of the system increases, however it is always linked to functional behavior as was described in the previous section.

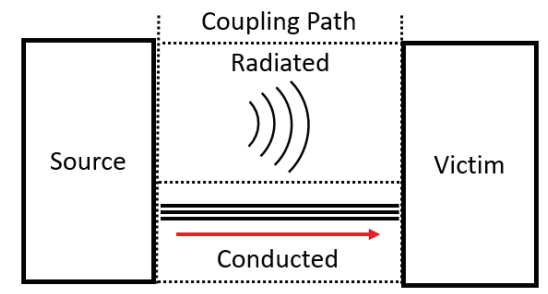

Figure 1.5: Basic decomposition of EMC coupling problem.

In the classical image of EMC one can identify a source, a victim and a coupling path (Figure 1.5). With respect to the investigated architecture, the individual SM is considered to be the source generating the possibly devastating interferences. Any source (e.g. photo-voltaic panel, battery, wind-turbine) or load (e.g. battery, motor, appliance) could be the victim. In case of the coupling path, two types of interference are identified, radiated and conducted 
phenomena. The objectives at which this thesis is aimed are related to identifying and possibly mitigating these conducted EMI, and radiated magnetic and electric fields based on:

1. Full wave models of M3C components and module

2. Behavioral circuit models for network simulation based on the full wave models

3. Experimental validation of the models

4. Optimized filters using novel nano-crystalline materials

5. EMI performance of the prototype

\subsection{Outline of the thesis}

This thesis is structured in line with the research goals stated in the previous section. Chapter 2 describes the origin of the generated noise. It starts with a theoretical analysis of the spectral content of a sinusoidal Pulse Width Modulation (sPWM) waveform, while eventually linking it to conducted and radiated noise arising in a single SM. It is then extrapolated to the multi-level system in Chapter 3 which incorporates a multitude of SMs. As can be seen in Figure 1.3 the switching modules can be connected in series as well as parallel. In the depicted medium voltage applications this will cause voltage and current switching behavior, inducing electric fields and magnetic fields respectively.

As the source is then identified, modeled and verified, mitigation techniques can be sought. Many mitigation techniques exist which focus on either the noise source or the propagation path [15]. Figure 1.6 shows an overview of known conducted EMI mitigation techniques. It is well known that the control scheme is directly related to the amount of EMI being generated [16-19], and many papers describe methods for reducing EMI inherently [20-22] or by optimizing the usage of parasitics [23-26]. Chapter 3 focuses on the switch control scheme at the source, while Chapter 4 focuses on applying passive filters in the propagation path and optimizing their design.

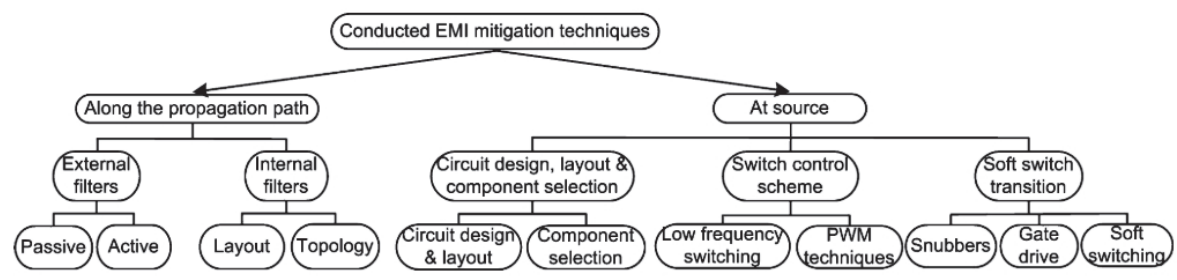

Figure 1.6: Mitigation techniques adopted from [15]. 
Chapter 3 will first introduce the developed sPWM generator that was used in the experimental setups described in this thesis. It is required for excluding the signal generation as a possible origin of any unexpected behavior at the high power side of the system. It also shows the constraints with which the $\mathrm{M} 3 \mathrm{C}$ demonstrator can be tested. As was mentioned, flexibility is a key feature as it allows modularity on which the system is based. The generator is developed to support at most eight SM's, while maintaining synchronization at high switching frequencies (up to $2 \mathrm{MHz}$ ). On the fly control through software without recompiling or uploading the bit-stream allows for fast and flexible testing scenarios. The carrier based sPWM generation is a relatively simple and known technique, and was implemented without optimizing for noise generation. Many control techniques exist, each with optimized features [9]. Implementation of these advanced control systems is omitted in this thesis as it does not represent the demonstrator as was created in [10]. Also research into implications for the functional behavior of the system is required and considered part of the project partners their objectives. Next to optimization of the generation of EMI, reduction of interference levels can still be achieved by designing low-impedance paths for disturbances away from the possible victims that have relatively low susceptibility levels $[14,27]$. In Chapter 4 the application of filters is discussed with emphasis on layout optimization through parasitic cancellation of coupling, and optimized Common Mode Choke (CMC) modeling. The analyses are performed through full wave $3 \mathrm{D}$ simulation techniques and back-annotating 3D field effects into lumped circuits which can be used in (2D) circuit simulators. For automated back annotation a generalized impedance to equivalent circuit fitting algorithm was implemented to determine optimal $R, L, C$ component values. It is mathematically shown that the algorithm potentially can fit an unlimited amount of components, in practice this is limited by computational power.

Chapter 5 describes novel EMI mitigation techniques, by combining the mitigation along the propagation path with mitigation at the source through the switch control scheme. It investigates the possibilities to reduce radiated as well conducted emissions in a sPWM controlled system. For conducted EMI optimization, it is assumed the system is implemented in its final stage and unknown parasitics are decreasing a filter's expected performance to inadequate levels. Being in control of switching frequencies, one can control the frequency components of the generated noise, thus allowing an optimal placement in-situ. Radiated EMI optimization relies on the same principle, however a technique is first developed for determining the effective radiation model that allows to asses optimal EMI placement.

Finally a summary per chapter is provided with conclusions in Chapter 6, followed by recommendations for further research. 


\section{Chapter 2}

\section{Noise source modeling}

In the introduction of this thesis a simplified description was given of a M3C implementation under investigation. Its functionality depends heavily on the switching behavior in each SM. This chapter presents the EMI analysis of single SM as it is the basic building block of the converter topology under study. The SM is a half-bridge consisting of two semiconductor switches, effectively having three legs at the power side and two on control side (Figure 2.1). Stacking SMs in series is done by interconnecting middle (Mid) leg with the lower (Low) leg of the next SM. This effectively means the lower leg is input while the middle one is the output. Evaluating the signal at the output (Mid) leg, will result in a voltage that either equals $V_{\text {low }}$ or $V_{\text {low }}+V_{\text {cap }}$.

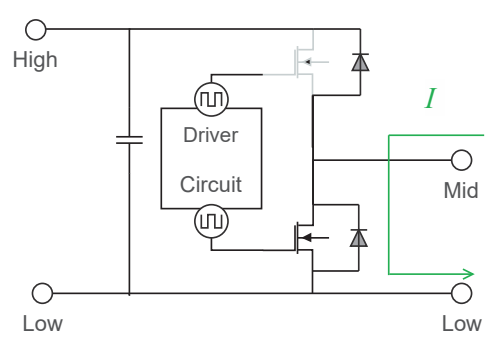

(a) Bypass capacitor

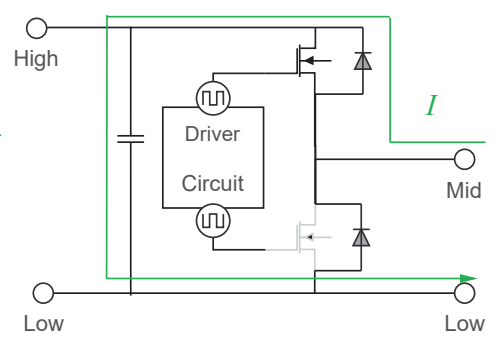

(b) Insert capacitor

Figure 2.1: Basic operational states of a half-bridge.

In case of multilevel converters, the control signals are generated using multi-carrier Pulse Width Modulation (PWM) techniques. A review on the operation, control and applications of the M2C is given in [9]. For the correct operation in the $\mathrm{M} 3 \mathrm{C}$ topology as described by [10], a multi-carrier scheme is required in combination with two reference signals to control the upper and lower arm of the converter. In Figure 2.2 several possible multilevel carrier

Parts of this chapter have been published in [28] and received the second best student paper award at EMC Europe 2018 in Amsterdam. 


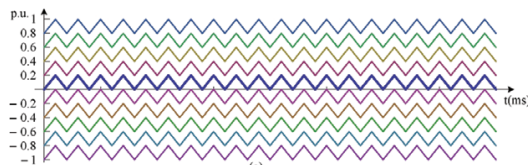

(a) Phase disposition

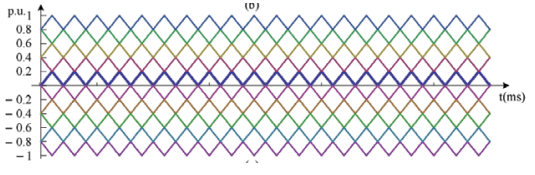

(c) Alternate phase opposition disposition

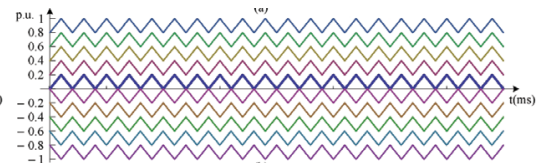

(b) Phase opposition disposition

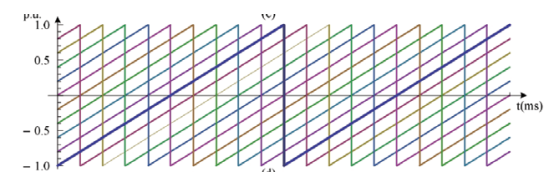

(d) Saw-tooth phase shifted

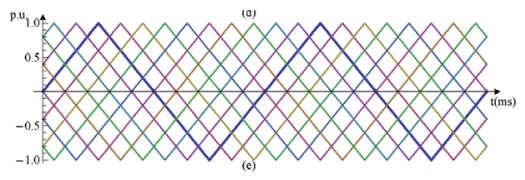

(e) Triangular phase shifted

Figure 2.2: Multi-level converter carrier schemes, adopted from [29].

generation schemes are shown. According to [29] the necessary voltage balancing at the flying capacitors can only be achieved by the schemes using the phase-shifted carriers shown in figures $2.2 \mathrm{~d}$ and $2.2 \mathrm{e}$, therefore in this thesis only the phase shifting schemes are used and investigated.

PWM generation is achieved by comparing a carrier wave with a reference waveform. The reference signal is therefore the 'wanted' signal. In case of the setup shown in Figure 1.4, the waveform inside the secondary power loop consists of sinusoidal modulated PWM, which is dubbed sPWM. This implies the reference waveform has to be a sinusoidal signal.

In short, each SM is controlled by an sPWM signal that is phase shifted according to the carrier waveform attributed to the individual SM. Under the assumption that the capacitor in the half-bridge is always charged (i.e. acts as an ideal voltage source) the SM operates as a conventional DC/AC-converter.

This chapter presents an EMI investigation of a half-bridge used as a DC/ACconverter. This is an imperative step in evaluating the EMI of the M3C prototype. It gives possibilities to mitigate EMI at the very origin, with a very broad and wide source of known solutions. The contribution of this chapter is firstly the simplified theoretical model for determining the output EMI spectrum of an sPWM waveform based on several functional, control parameters that were identified in the work of [10]. Secondly the contribution of this chapter is the conducted and radiated EMI analysis of a very fast switching Galium-Nitride ( GaN) based half-bridge. 


\subsection{Theoretical Analysis of sPWM}

sPWM is a digital modulation technique that uses a high frequency pulse train, to approximate a low frequency reference voltage or current. The desired output wave is generated by comparing a reference wave to a carrier wave. While a lot of different forms of sPWM have been developed in the past, they are often slightly different variations. Six basic variations of two level sPWM will be investigated for this model [30]:

1. unipolar trailing-edge modulation

2. bipolar trailing-edge modulation

3. unipolar leading-edge modulation

4. bipolar leading-edge modulation

5. unipolar double-edge modulation

6. bipolar double-edge modulation

Where leading and trailing edge modulation schemes use a sawtooth shaped carrier and double edge modulation schemes use a triangular shaped carrier. Although the mathematical representation of these six modulation types are very different, their properties have commonalities:

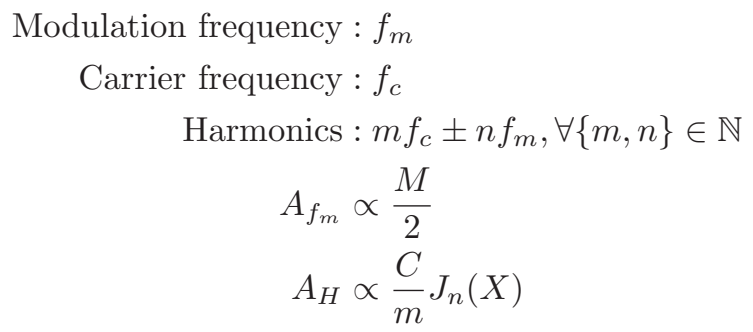

where:

$\dot{A}_{\text {fm }}=$ Amplitude of the modulation frequency

$A_{H}=$ Amplitude of the harmonics

$C=$ Amplitude factor

$M=$ Modulation index

$J_{n}(X)=$ The Bessel function of the first order for positive or integer values of $n$

$X=$ The argument of the Bessel function

$m=$ carrier harmonic number

$n=$ sub-harmonic number

$M=$ modulation index

In [30] the modulation index is defined as:

$$
M=\frac{2 \cdot A_{f m}}{A_{p-p, \text { carrier }}}
$$


In case of the sPWM generator designed for this research: $A_{p-p, c a r r i e r}$ is the peak to peak amplitude of the carrier signal, which is set to 1 . The amplitude of the modulation frequency $\left(A_{f m}\right)$ is normalized with respect to the value of $A_{p-p, \text { carrier }}$, which implies values of $A_{f m}$ between 0 and 0.5 .

Figure 2.3 shows an example of an amplitude spectrum resulting from an sPWM signal. In case of the sPWM signal (and its inverse) is used for controlling two switches in a single half-bridge, one has the basics for creating a $\mathrm{DC} / \mathrm{AC}$ converter. In the $\mathrm{DC} / \mathrm{AC}$ converter setup, $f_{m}$ can be considered to be the wanted AC output signal, whereas the rest of the signals are considered to be EMI and related to the used pulse train for generating the output. The goal of the model is to approximate all the carrier frequency harmonics and sub-harmonics. This is done by estimating the maximum amplitude and bandwidth of each band of harmonics. A band of harmonics is considered to be a single carrier harmonic together with its sub-harmonics $\left(i f_{c} \pm j f_{m}\right.$, with $i$ denoting the band under consideration).

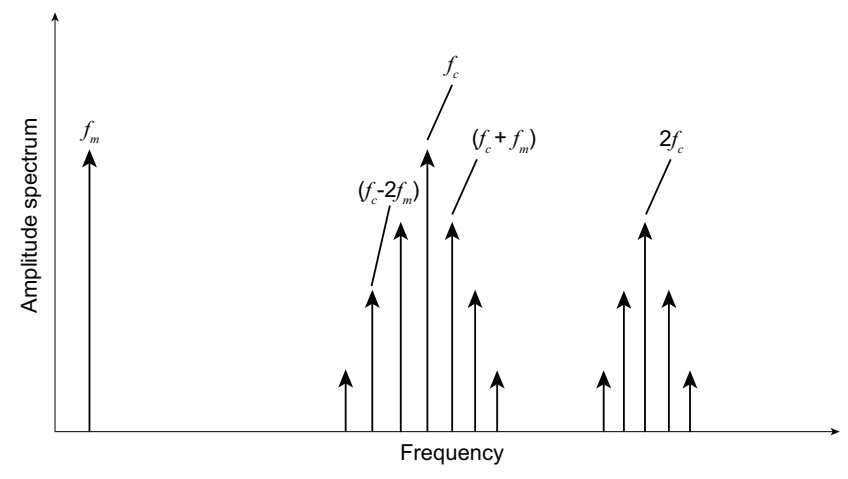

Figure 2.3: Example of a sPWM spectrum.

\subsubsection{Amplitude}

As indicated in the previous section the amplitude of the harmonics are proportional to the Bessel function and a constant amplitude factor.

$$
A_{H} \propto \frac{C}{m} J_{n}(X)
$$

Here $C$ is the amplitude factor and $J_{n}(X)$ is the Bessel function of the first order for positive integer values of $n$. The amplitude factor is dependent on the power supply voltage, which can be unipolar or bipolar. A peak to peak voltage is used for the DC power supply, as a sPWM wave switches between plus and minus the supply voltage or between zero and the supply voltage. The argument of the Bessel function is dependent on the carrier harmonic number, modulation index and a correction factor for different types of modulation 
techniques. In the resulting equation, for leading- or trailing-edge modulation $k=1$ and for double-edge modulation $k=2$ :

$$
C=\frac{k \cdot V_{p-p}}{\pi}, \quad X=\frac{m \pi M}{k}
$$

where:

$$
\begin{aligned}
& V_{p-p}=\text { peak to peak, DC }, \text { supply voltage } \\
& m=\text { carrier harmonic number } \\
& n=\text { sub-harmonic number } \\
& M=\text { modulation index }
\end{aligned}
$$

Since $X$ is proportional to $m$ and for $m \rightarrow \infty$, it can be concluded that $X \rightarrow \infty$. Therefore it is assumed that $J_{n}(X)$ can be approximated by the envelope of the asymptote of the Bessel function which can be described when $n$ is fixed and $|X| \rightarrow \infty[31]$ with:

$$
J_{n}(X) \approx \sqrt{\frac{2}{\pi X}}
$$

Bessel functions with $n=0, \ldots, 3$ and the proposed asymptote are shown in Figure 2.4. This figure shows that the approximation follows the envelope of the Bessel functions nicely after the first local maximum. Combining the approximations for the amplitude factor $(C)$ and the Bessel function $\left(J_{n}(X)\right)$ results in Equation 2.5, which can be used for the estimation of the amplitude of the side harmonics.

$$
A_{H} \approx \frac{k V_{p-p}}{m \pi} \sqrt{\frac{2 k}{m \pi^{2} M}}
$$

Where $k=1$ for leading- or trailing-edge modulation and $k=2$ for double-edge modulation.

\subsubsection{Bandwidth}

The bandwidth of a sub-harmonic band is equal to the area in which most of the power is concentrated. The amount of power within a certain bandwidth is proportional to the amount of side harmonics that are taken into account, where the amplitude of the sub-harmonics is proportional to $J_{n}(X)$. To determine this bandwidth, the spectrum of one carrier frequency and its band of sub-harmonics can be compared to the passband spectrum of a Frequency Modulation (FM) wave. Equation 2.6 shows the discrete spectrum for FM. The spectrum consists of a number of peaks spaced at $f_{c} \pm n f_{m}$ where $n=0,1, \ldots, \infty$ whose amplitudes are proportional to $\frac{A_{c}}{2} J_{n}(\beta)$.

$$
S_{F M}=\frac{A_{c}}{2} \sum_{-\infty}^{\infty} J_{n}(\beta)\left[\delta\left(f-f_{c}-n f_{m}\right)+\delta\left(f+f_{c}+n f_{m}\right)\right]
$$




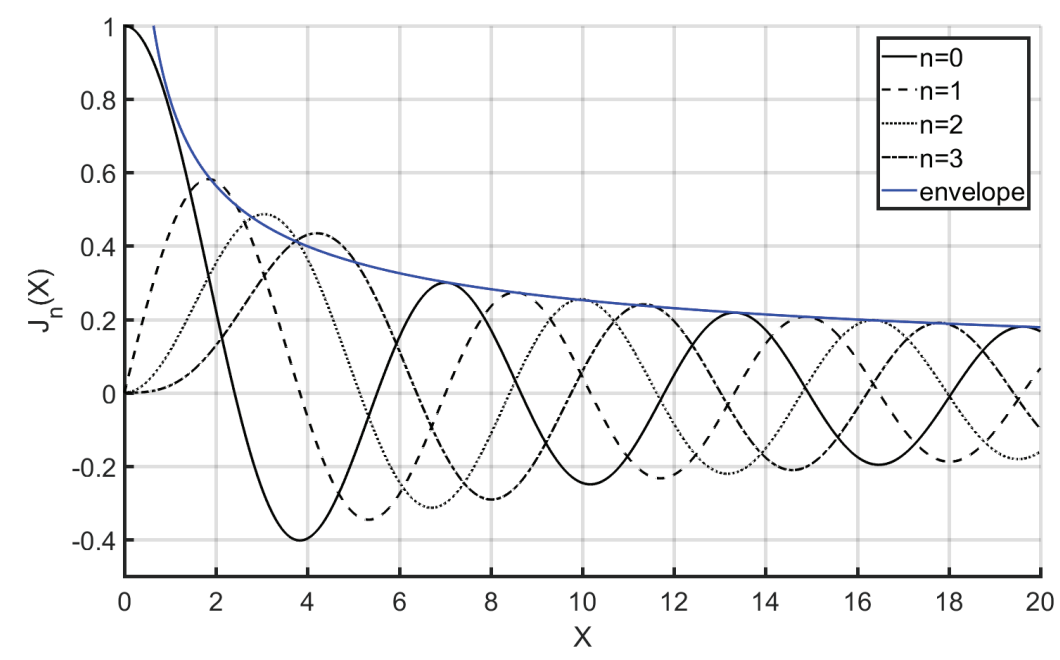

Figure 2.4: Envelope of the asymptote of the Bessel function.

Carson's rule estimates the bandwidth in which $98 \%$ of the power from the carrier is located for FM signal. In [32] it is defined as:

$$
B_{F M} \approx 2\left(f_{m} \beta+f_{m}\right)
$$

Since FM sinusoids and sPWM both have their energy spread proportional to harmonics weighed by Bessel functions, it is assumed that Carson's rule is also applicable here for estimating the bandwidth of one band of side harmonics. Noticing that the argument of the Bessel function for FM is $\beta$ [32] while it is $X$ for sPWM, they can be substituted. Which results in the bandwidth estimation shown in 2.8 .

$$
B_{s h} \approx \frac{2}{k} f_{m} m \pi M+2 f_{m}
$$

Here $B_{s h}$ is denoting the bandwidth of sub-harmonics, and again $k=1$ for leading- or trailing-edge modulation and $k=2$ for double-edge modulation.

\subsubsection{Model}

As the estimation of bandwidth and amplitude holds for a single carrier harmonic with its sub-harmonics, thus a single harmonic band, one can extrapolate the results to include all harmonic bands. This is done by using the property that a rectangular pulse train comprises of the superposition of all harmonics of the fundamental (i.e. the carrier frequency), which is shown by the Fourier series. The sPWM spectrum has the harmonic bands located around the carrier harmonics at $m \cdot f_{c}$ where $m=1,2, \ldots, \infty$. Each band will therefore be plotted using a rectangular function centered at the corresponding carrier harmonic. 
The width and the height of a rectangular function is equal to the predicted bandwidth and amplitude of that band. This results in $m$ rectangular functions, one for each harmonic band. Combining these three properties results in the final model shown in Equation 2.9.

$$
S_{s P W M} \approx \sum_{m=1}^{\infty} A_{H} \operatorname{rect}\left(\frac{f-m f_{c}}{B_{s h}}\right)
$$

Substituting Equations 2.5 and 2.8 into Equation 2.9 results in:

$$
S_{s P W M} \approx \sum_{m=1}^{\infty} \frac{k V_{p-p}}{m \pi} \sqrt{\frac{2 k}{m \pi^{2} M}} \operatorname{rect}\left(\frac{f-m f_{c}}{\frac{2}{k} f_{m} m \pi M+2 f_{m}}\right)
$$

Now the theoretical model has been derived, the next sections will evaluate the GaN half-bridge in operation. This is first done in a conducted EMI test setup followed by a radiated electric field test based on CISPR 16-2-3 standard. These tests were both performed at Thales Hengelo in a semi-anechoic chamber. After that the Device Under Test (DUT) was moved into the laboratory of the university of Twente and a time efficient magnetic field measurement was performed using the technique developed in [33]. This is a time domain measurement of the magnetic field according to the MIL-STD RE101 in which case the Digital Signal Processing (DSP) effectively replaces the traditional EMI test receiver function.

\subsection{Conducted Emissions}

The validity of the model is experimentally verified by measuring sPWM signals and comparing those to the predicted spectra of the model. The device under test (DUT) consists of a Field-Programmable Gate Array (FPGA) based sPWM generator controlling a GaN half-bridge to drive a resistive load. The development and implementation of the FPGA based sPWM generator is discussed in Chapter 3. The used setup allows for measuring the sPWM control signal, and the output signal. Figure 2.5 shows a picture of the DUT without a source and load. One of the advantages in using an FPGA based sPWM generator is the flexibility it offers. The design specifications can be altered by changing the design parameters and reprogramming the FPGA. The developed generator allows to freely adjust parameters related to the M3C prototype identified in [10]:

1. Carrier Frequency $\left(f_{c}\right)$

2. Modulation Frequency $\left(f_{m}\right)$

3. AC-amplitude $\left(m_{i}\right)$

4. DC-offset $\left(D_{i}\right)$ 


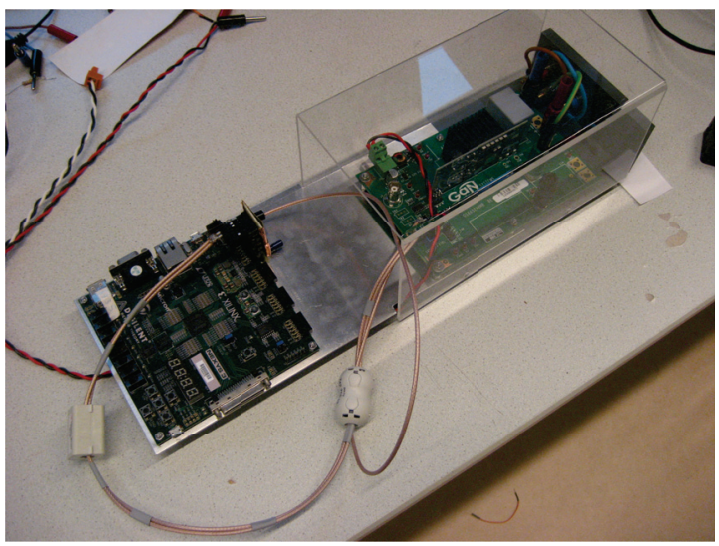

Figure 2.5: The DUT, consisting of a sPWM generator (left) and half-bridge (right). The load can be connected to the panel seen on the right.

5. Dead-time $\left(t_{\text {dead }}\right)$

6. Number of levels (set carrier phase shifts)

The AC-amplitude and DC-offset are parameters of the sinusoidal reference signal. The AC-amplitude is related to the derived mathematical model for spectral estimation. Due to $A_{p-p, \text { carrier }}=1$, the modulation index is effectively $M=2 \cdot A_{f m}=2 \cdot m_{i}$. The EMI characterization is highly dependent on the used parameters. So in this measurement, $f_{c}$ is varied between $24.4 \mathrm{kHz}$ and $1 \mathrm{MHz}, f_{m}$ is varied between $1 \mathrm{kHz}$ and $100 \mathrm{kHz}$ while not exceeding $f_{m}<\frac{f_{c}}{10}$, $M$ is varied from $\frac{1}{4}$ to $1, D_{i}$ is set to be 0.5 and $t_{\text {dead }}$ is set to $50 \mathrm{~ns}$. Only bipolar double-edge modulated sPWM is generated. The half bridge module is a Commercial Of The Shelf (COTS) available evaluation kit created by GaN systems. The half-bridge module consists of the GS665MB-EVB evaluation platform accommodating the GS66508B-EVBDB daughter board. The switches used have typical rise and fall times of $\approx 10 \mathrm{~ns}$. The half bridge is loaded by one of two different loads, where $Z_{1}=27.2 \Omega+7.1 \mu \mathrm{H}$ and $Z_{2}=10.2 \Omega+26 \mu \mathrm{H}$. Figure 2.6 shows a detailed schematic of the setup where:

$$
\begin{aligned}
& \text { DUT }=\text { The test system } \\
& \text { V1 = Profilter SP430 power supply } \\
& \text { V2 = V3 = Delta SM 7020-D power supplies set at } 35 \mathrm{~V} \text { DC } \\
& \text { Load = One of the two tested loads }
\end{aligned}
$$

\subsubsection{Conducted Emission Results}

The different measurement waveforms are shown in Figure 2.7. In black the recorded SPWM is displayed, while in red an ideal filtered version is shown. 


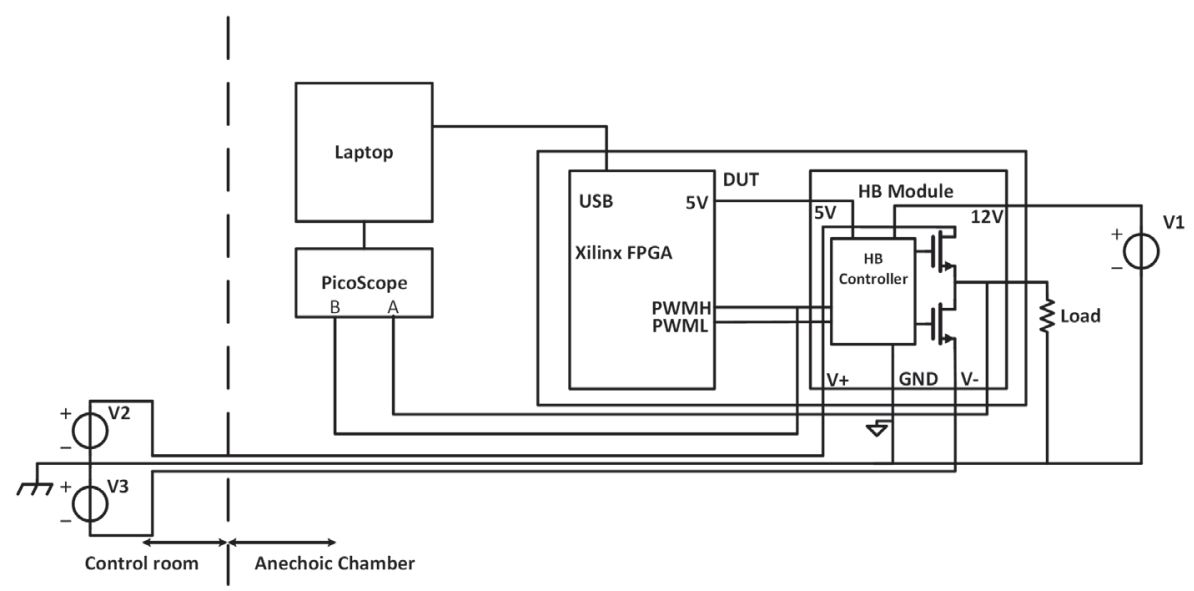

Figure 2.6: Schematic representation of the used measurement setup for the sPWM and half bridge outputs. Cables from the source are coaxially shielded cables.

The filter passes the low frequency modulation wave, while blocking the harmonic bands produces by the switching behavior at the carrier frequencies. Comparing Figure 2.7a and Figure 2.7b shows that the modulation amplitude is directly related to the amplitude of the resulting $\mathrm{AC}$ output. Note that the figures shown only the first $2 \mathrm{~ms}$ of the recorded $100 \mathrm{~ms}$.

First, to validate the simplified model from Equation 2.10, a measured conducted spectrum together with the predicted spectrum from the model is shown in Figure 2.8. The results of a single set of frequencies are shown (i.e. $f_{c}=24.4 \mathrm{kHz}$ and $f_{m}=1 \mathrm{kHz}$ ), while in the measurements a large amount of frequency combinations have been measured. It takes a very long time to measure all possible configurations, when also combined with different combinations of modulation indexes, dead-times and DC-offsets.

From Figure 2.8 it can be seen that the model is over estimating the lower frequencies $(f<100 \mathrm{kHz})$ while it is under estimating the higher frequencies $(f>100 \mathrm{kHz})$. The variation seen here is within $3 \mathrm{~dB}$ for the first 25 harmonics. Next to this, the increase in bandwidth at a harmonic can be seen, when the sub-harmonics become more dominant. The results show the model is adequately predicting the conducted EMI behavior. Therefore, the theoretical model is considered to be a useful tool for estimating the expected conducted EMI generated in a single SM. 


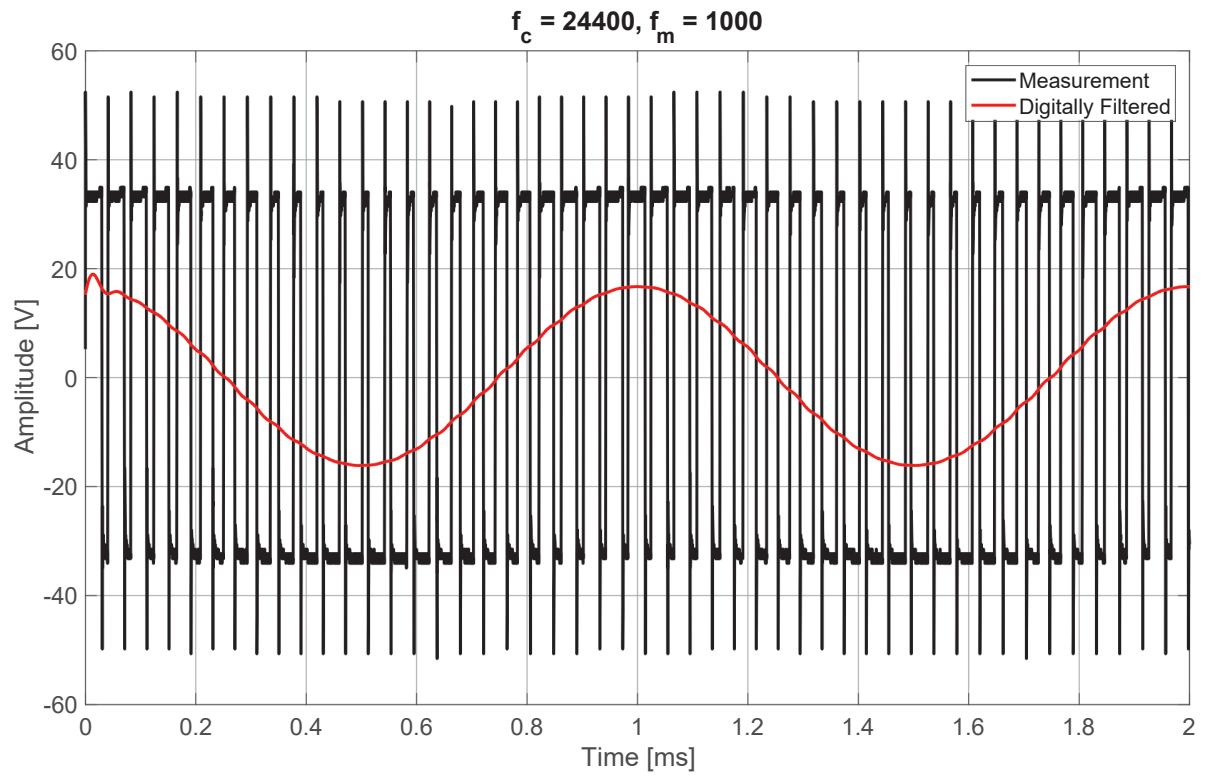

(a) Modulation index of 0.5

$f_{c}=24400, f_{m}=1000$

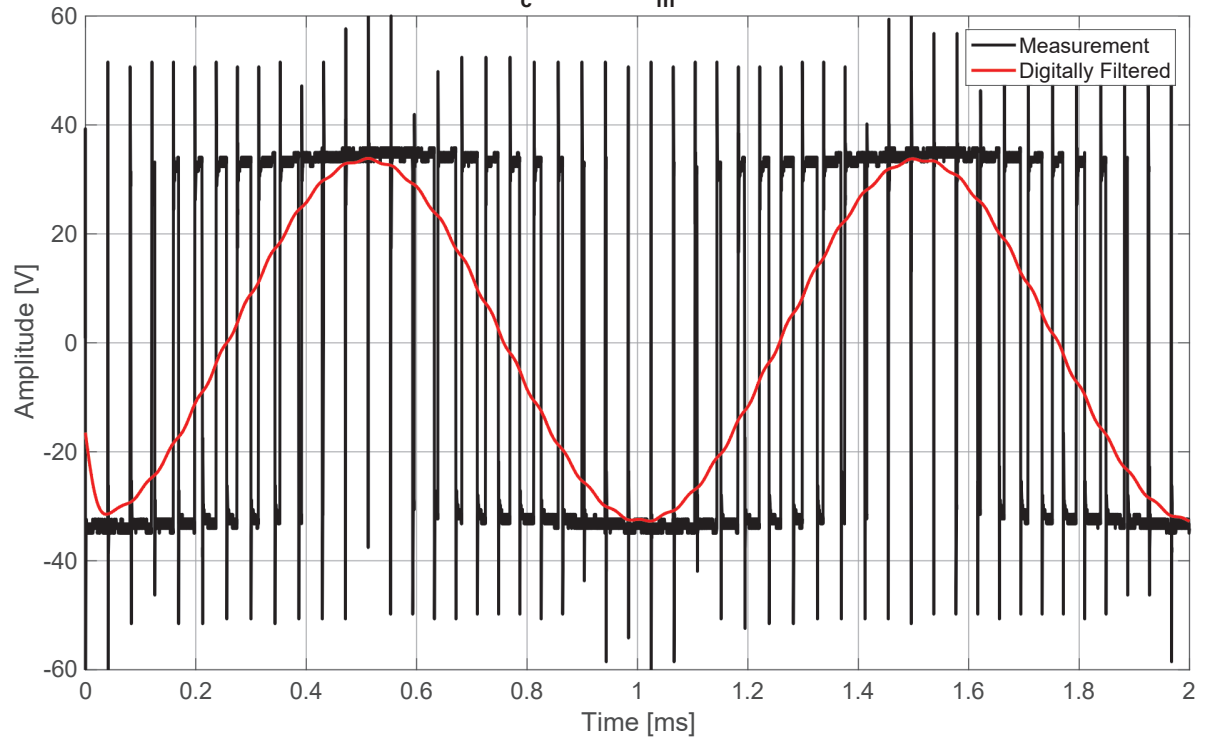

(b) Modulation index of 1

Figure 2.7: Time domain measurements recorded during the conducted measurement tests are shown in black. In red a digitally (almost ideally) filtered version is shown, which demonstrates the sinusoidal waveform resulting from the sPWM. The filtered version is shifted with the group delay of the implemented FIR filter. 


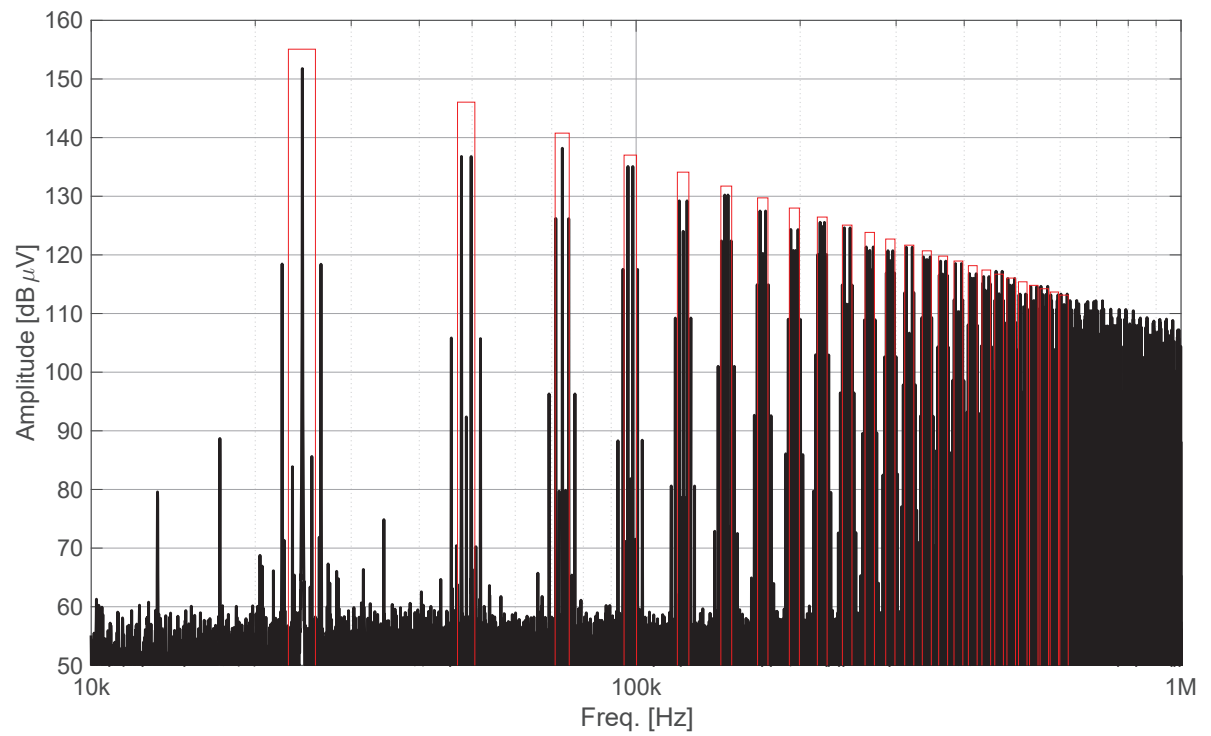

(a) Modulation index of 0.25

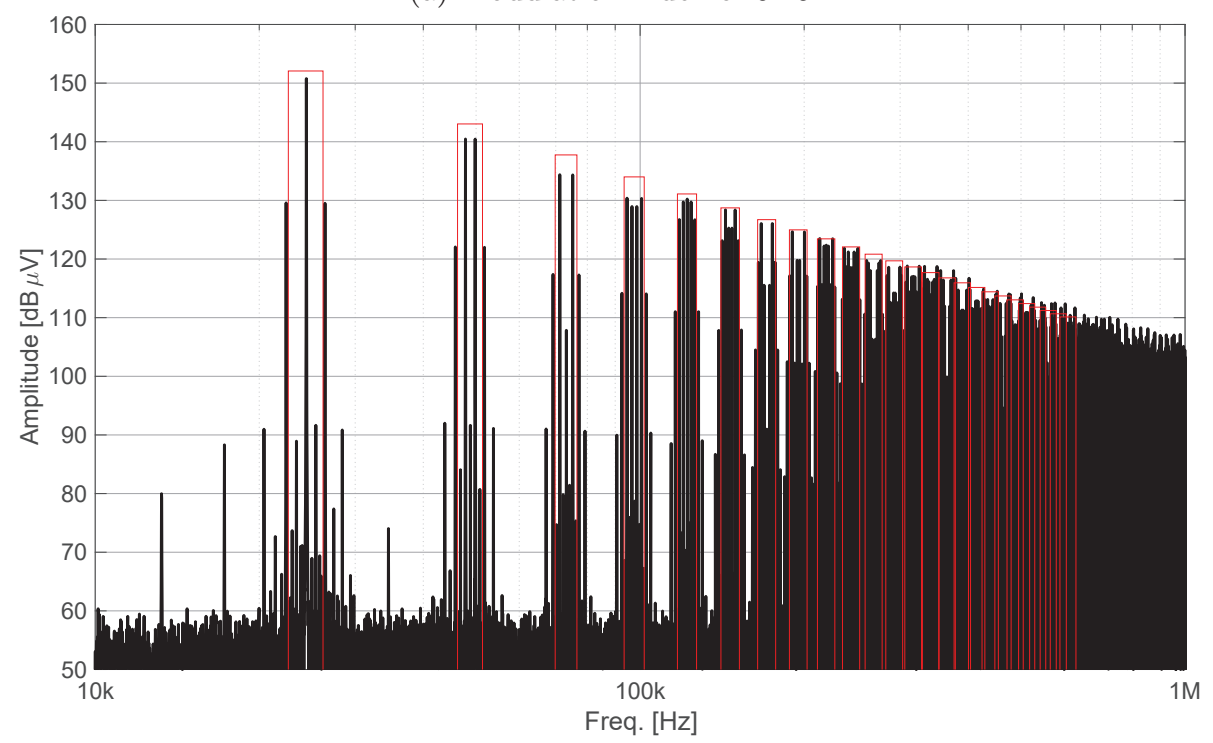

(b) Modulation index of 0.5

Figure 2.8: Measured conductive spectrum plotted with their respected theoretical harmonic bands for different values of modulation index. The spectra plotted are for a carrier frequency of $24.4 \mathrm{kHz}$ with a modulation frequency of $1 \mathrm{kHz}$. 


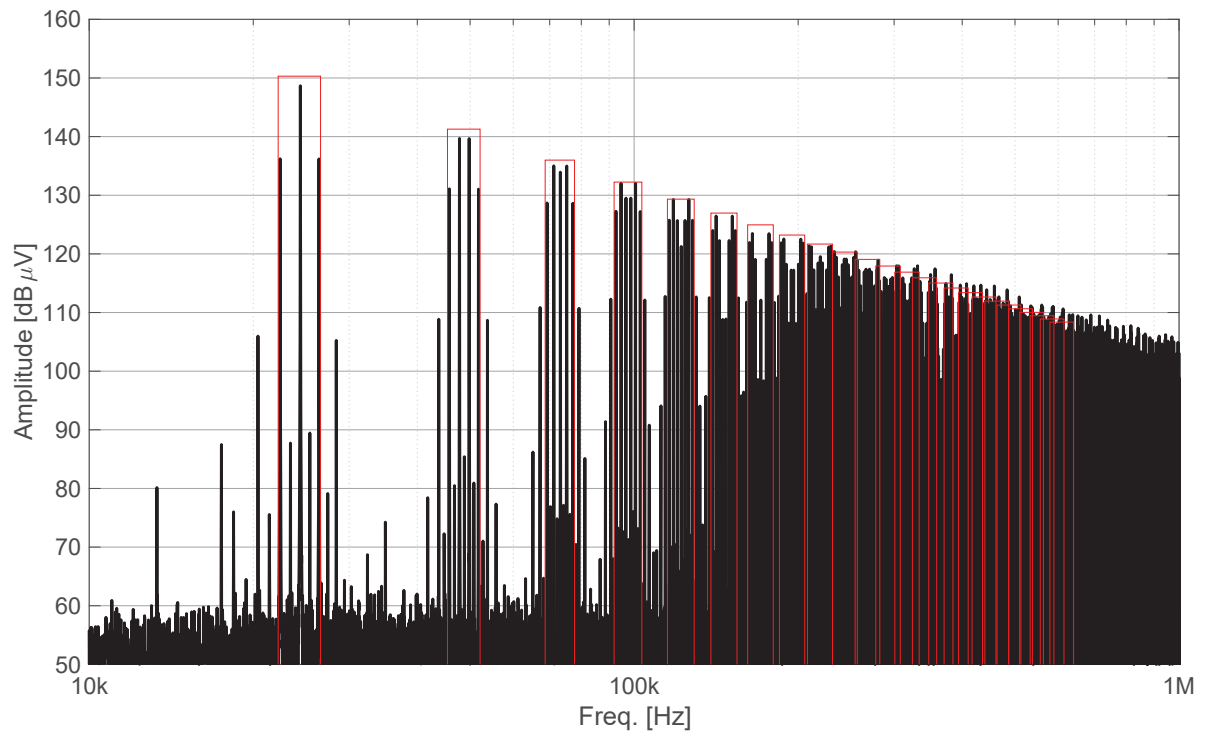

(c) Modulation index of 0.75

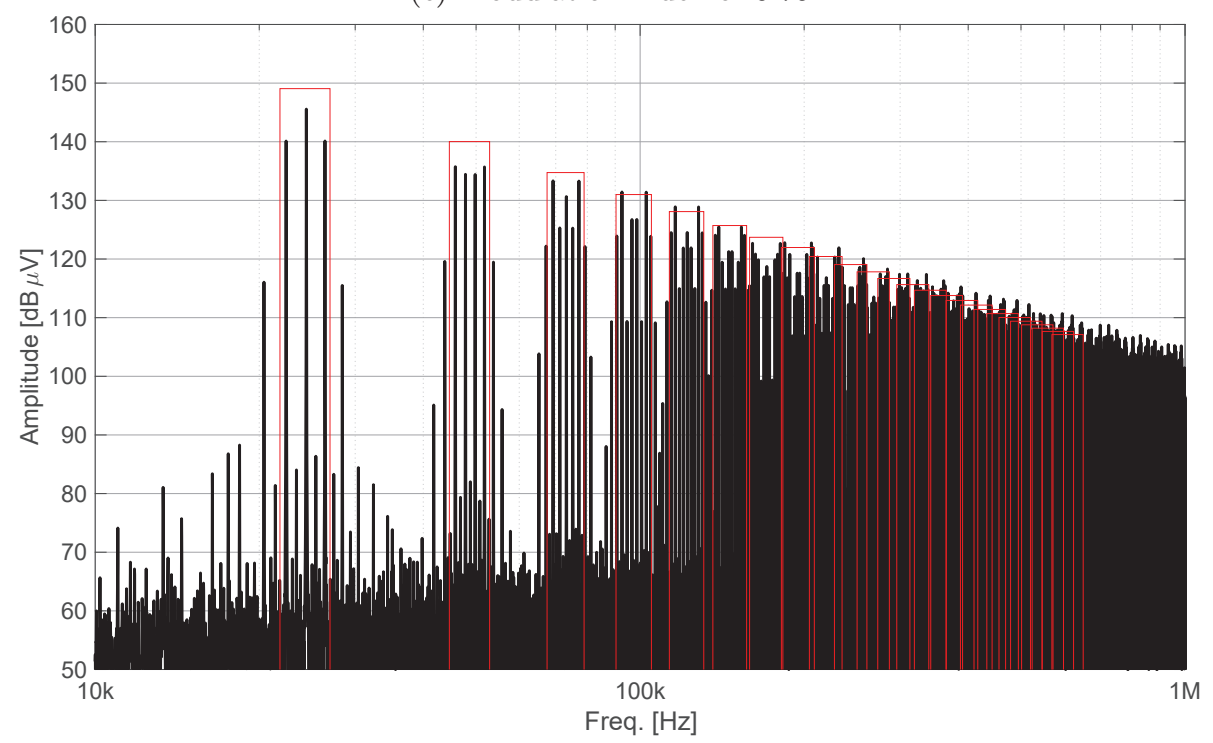

(d) Modulation index of 1

Figure 2.8: Measured conductive spectrum plotted with their respected theoretical harmonic bands for different values of modulation index. The spectra plotted are for a carrier frequency of $24.4 \mathrm{kHz}$ with a modulation frequency of $1 \mathrm{kHz}$. 


\subsection{Radiated Emissions}

Interference from fields can be split into two main contributors when considering low-frequency (approximately $f<30 \mathrm{MHz}$ ) radiation. The electric field and magnetic field can be assumed weakly coupled in this region. However it is of interest in power electronics, as the generated currents and voltages contain frequency components in this bandwidth. A relation between the studied emitted fields and conducted EMI is found. First electric field is treated, as the setup is identical to the conducted emission measurement setup, apart from the measurement device now using an antenna.

\subsubsection{Radiated - Electrical Field}

In this section the electric field emitted is studied. Due to the high switching voltages and stray capacitances between the components and eventually between the stacked SMs, EMI risks are expected in the fundamental implementation. To model and simulate the fields originating from a single SM, measurements were performed under varying operating conditions.

Basically the setup used in the conducted EMI tests was slightly altered to perform electrical field strength measurements. Comparison of Figure 2.6 and Figure 2.10 shows the laptop and oscilloscope are moved outside the anechoic chamber, while an antenna is placed within. The measurement setup is shown in Figure 2.9. The distance between the DUT and the antenna is $3 \mathrm{~m}$, the height of the table is $75 \mathrm{~cm}$. The radiated signals were measured in time domain using a Pico Technology PicoScope 2208B using a measurement time of $100 \mathrm{~ms}$. The recorded time domain signals are processed as described in appendix $\mathrm{C}$, which results the corresponding frequency spectrum. The measurement bandwidth spans from $1 \mathrm{kHz}$ to $100 \mathrm{MHz}$, due to limitations of the antenna and measurement bandwidth of the PicoScope. However the DUT operates within this bandwidth, thus the measurement setup is considered adequate for characterization.

Where

$$
\begin{aligned}
& \mathrm{A} 1=\text { A.R.A SAS-2/B measurement antenna } \\
& \mathrm{DUT}=\text { The test system } \\
& \mathrm{V} 1=\text { Profilter SP430 power supply } \\
& \mathrm{V} 2=\mathrm{V} 3=\text { Delta SM } 7020-\mathrm{D} \text { power supplies set at } 35 \mathrm{~V} \text { DC } \\
& \text { Load }=\text { One of the two tested loads }
\end{aligned}
$$

\subsubsection{Electric Field Results}

In the classical EMC emission test one tests an object to determine whether it is compliant with the appropriate standards. Note however in this case the DUT 


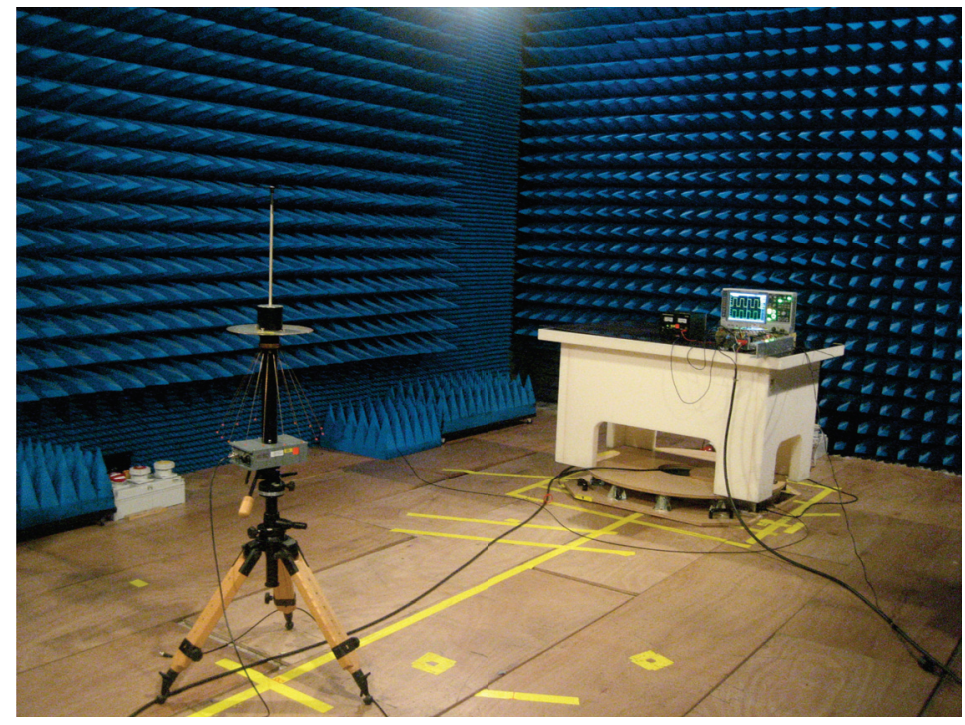

Figure 2.9: Measurement setup inside the anechoic chamber, the oscilloscope is for monitoring outside the anechoic chamber.

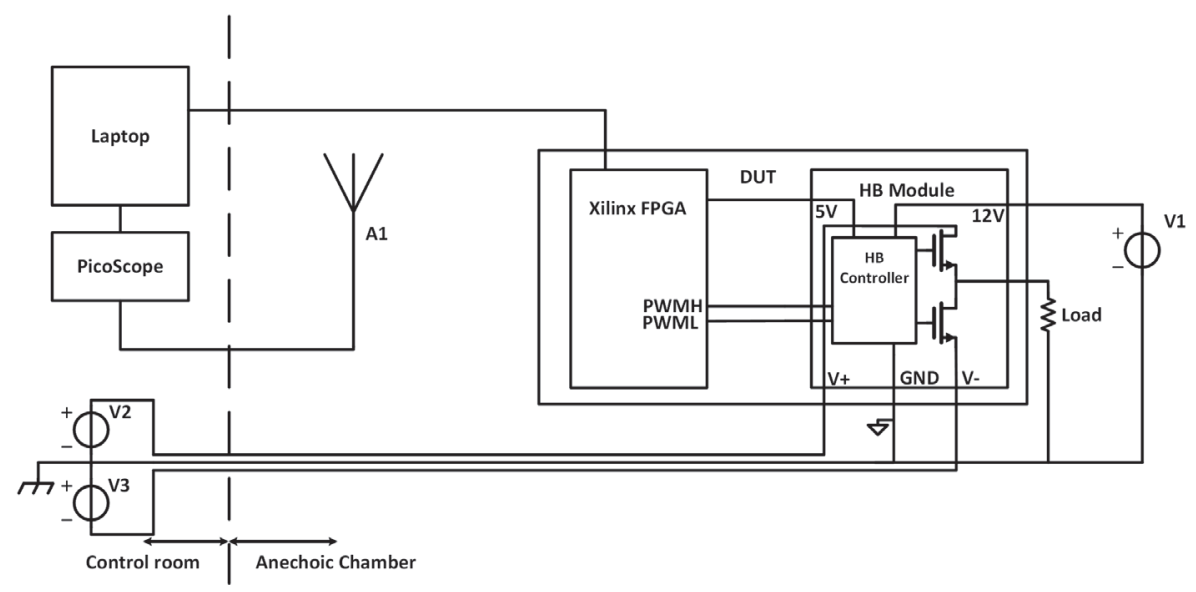

Figure 2.10: Schematic representation of the used measurement setup for the radiated emission test.

is not tested for compliance, but its emissions are analyzed for dependencies on control parameters. An example of the measured radiated spectra is shown in Figure 2.11 together with Figure 2.12, which zooms in on the 1st, 2nd and 3rd harmonic. The acquired spectra is for a single set of parameters, again with a varying modulation index. 


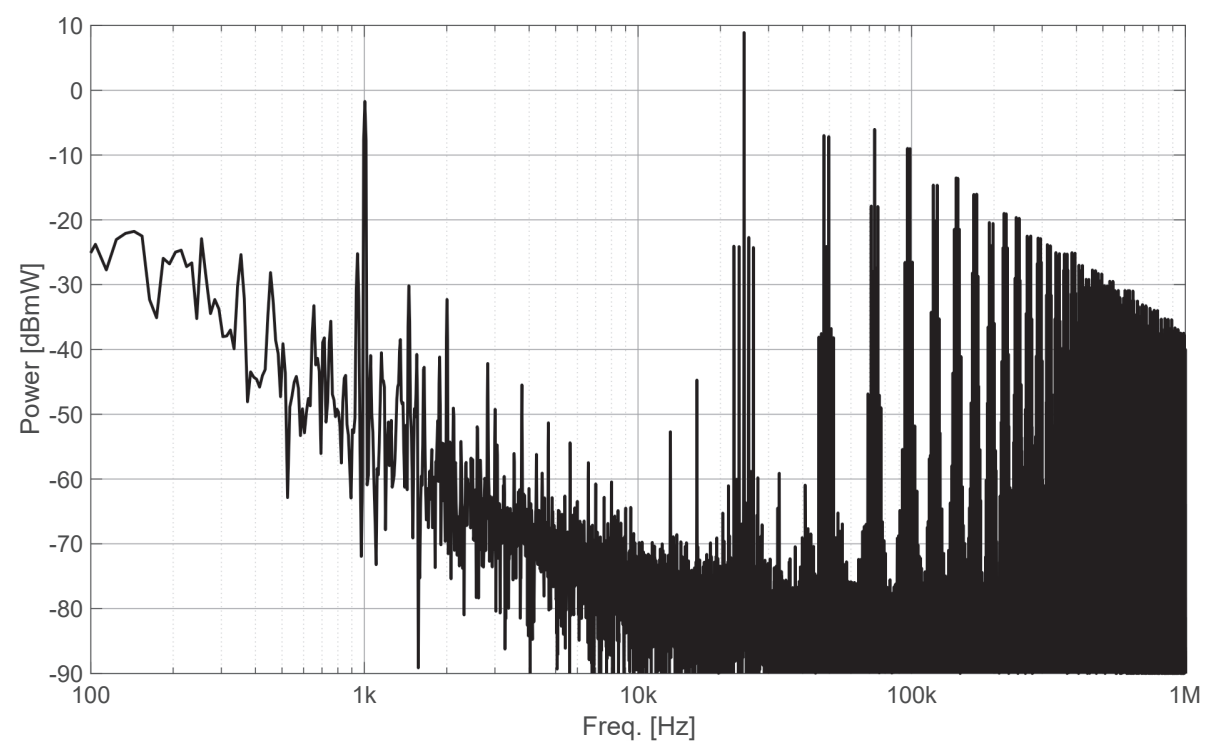

(a) Modulation index of 0.25

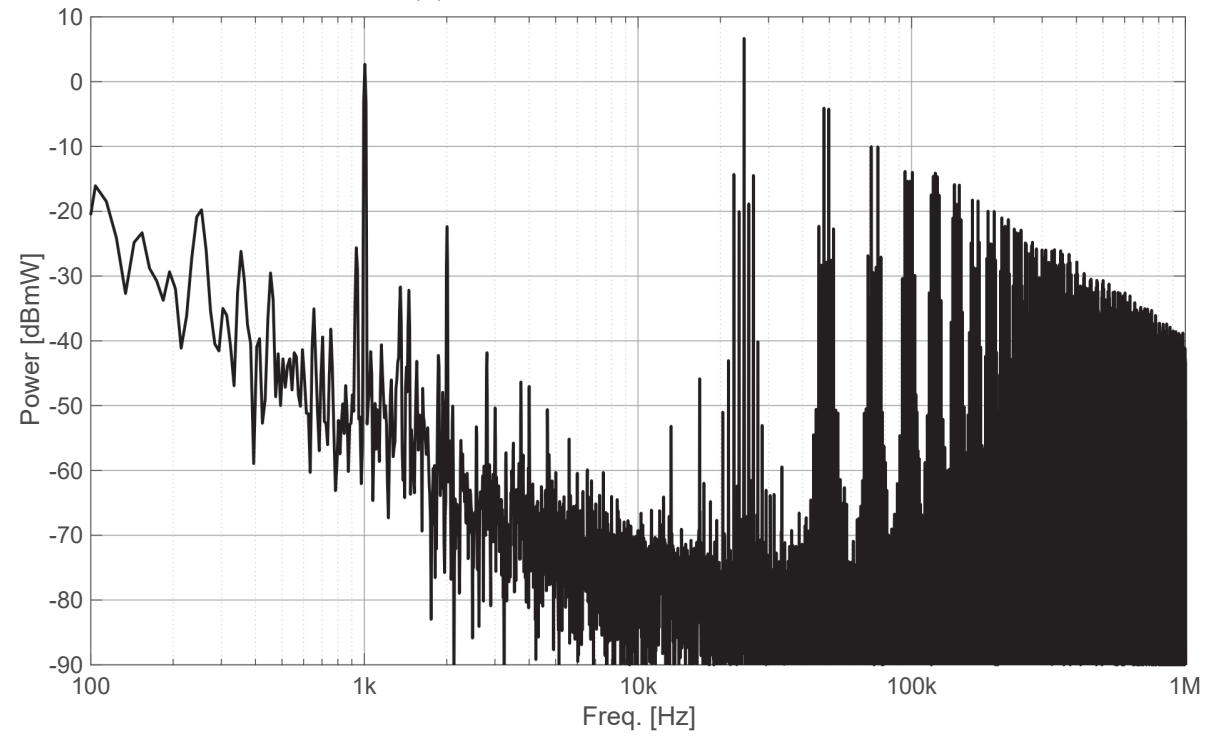

(b) Modulation index of 0.5

Figure 2.11: Measured radiative spectrum for different values of modulation index. The spectra plotted are for a carrier frequency of $24.4 \mathrm{kHz}$ with a modulation frequency of $1 \mathrm{kHz}$. 


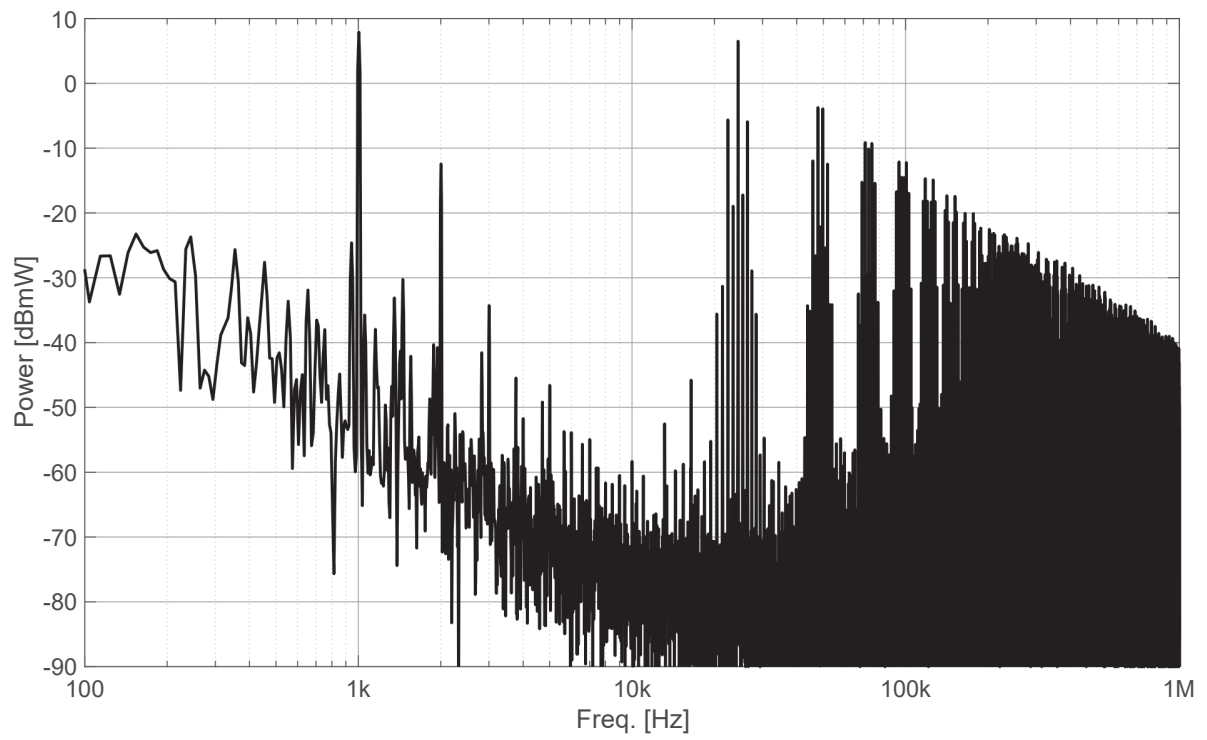

(c) Modulation index of 0.75

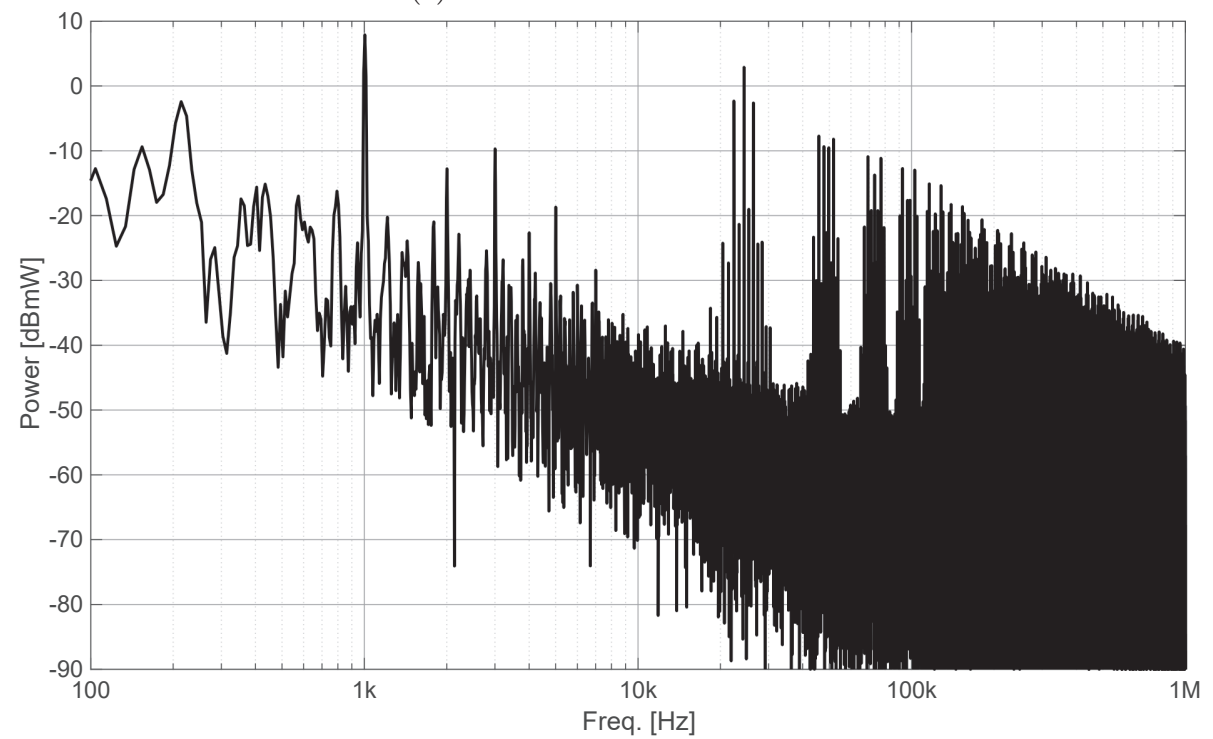

(d) Modulation index of 1

Figure 2.11: Measured radiative spectrum for different values of modulation index. The spectra plotted are for a carrier frequency of $24.4 \mathrm{kHz}$ with a modulation frequency of $1 \mathrm{kHz}$. 


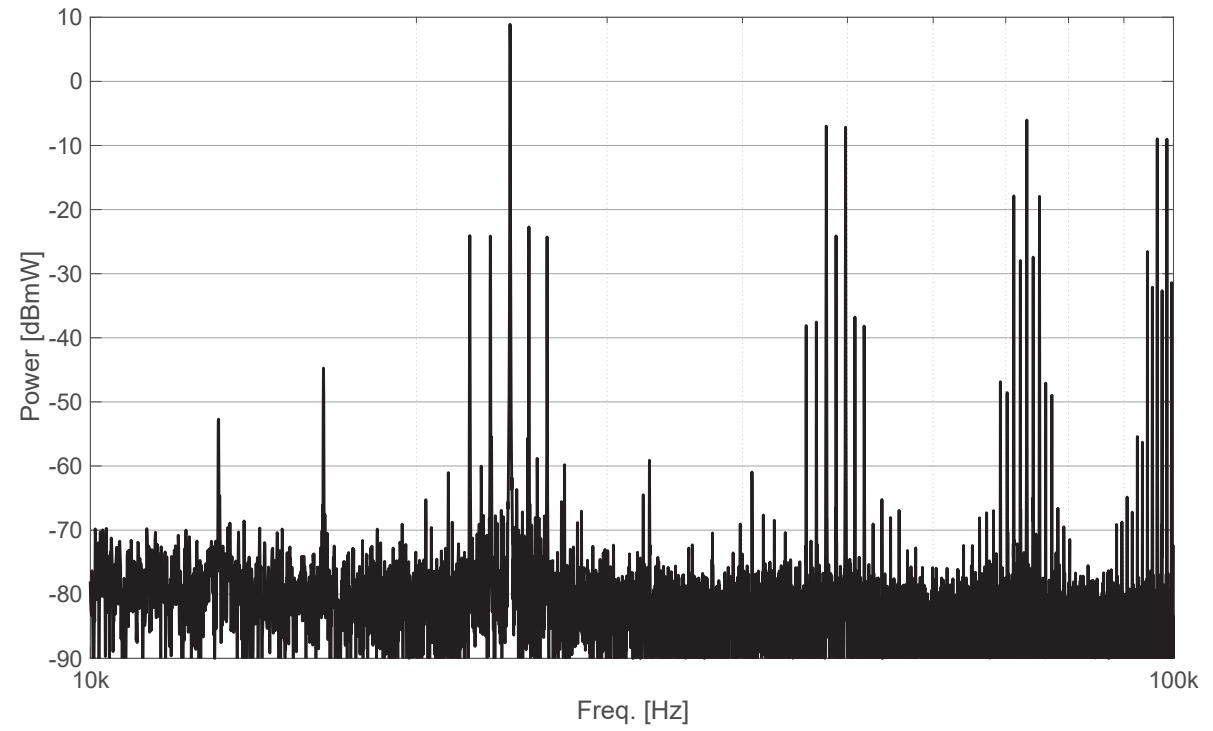

(a) Modulation index of 0.25

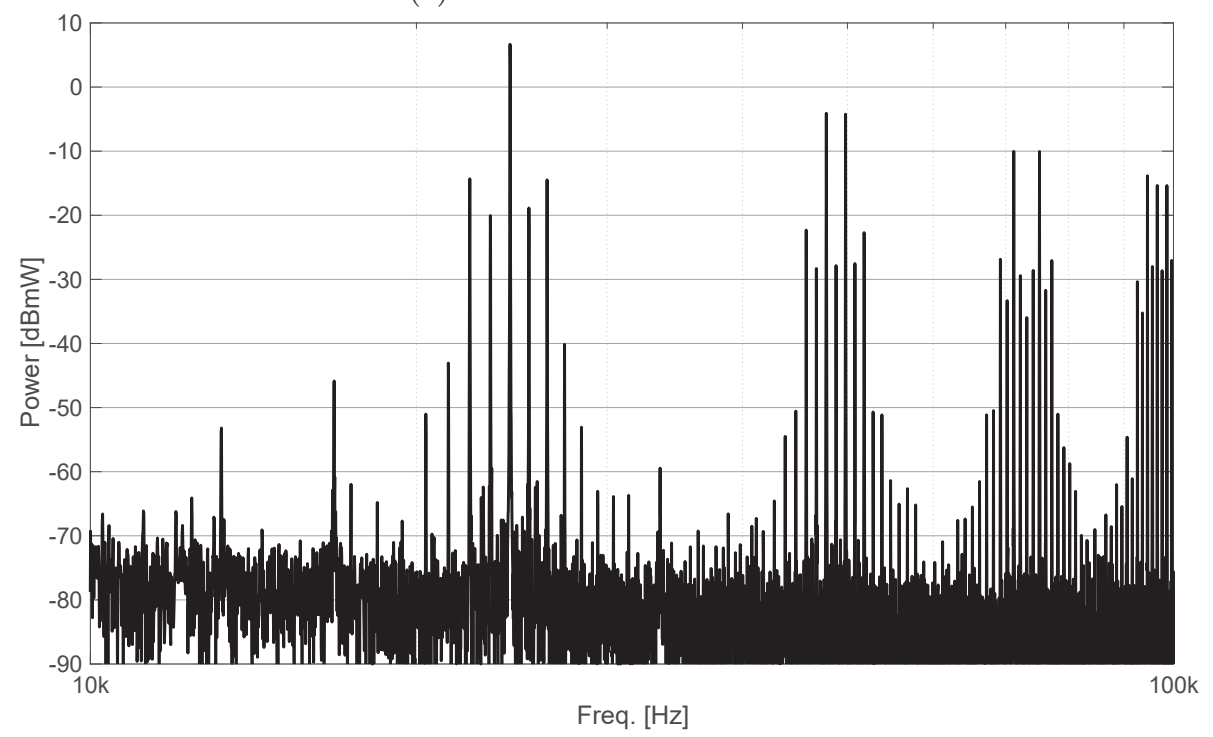

(b) Modulation index of 0.5

Figure 2.12: Zoomed in versions of the radiative spectra in Figure 2.11. 


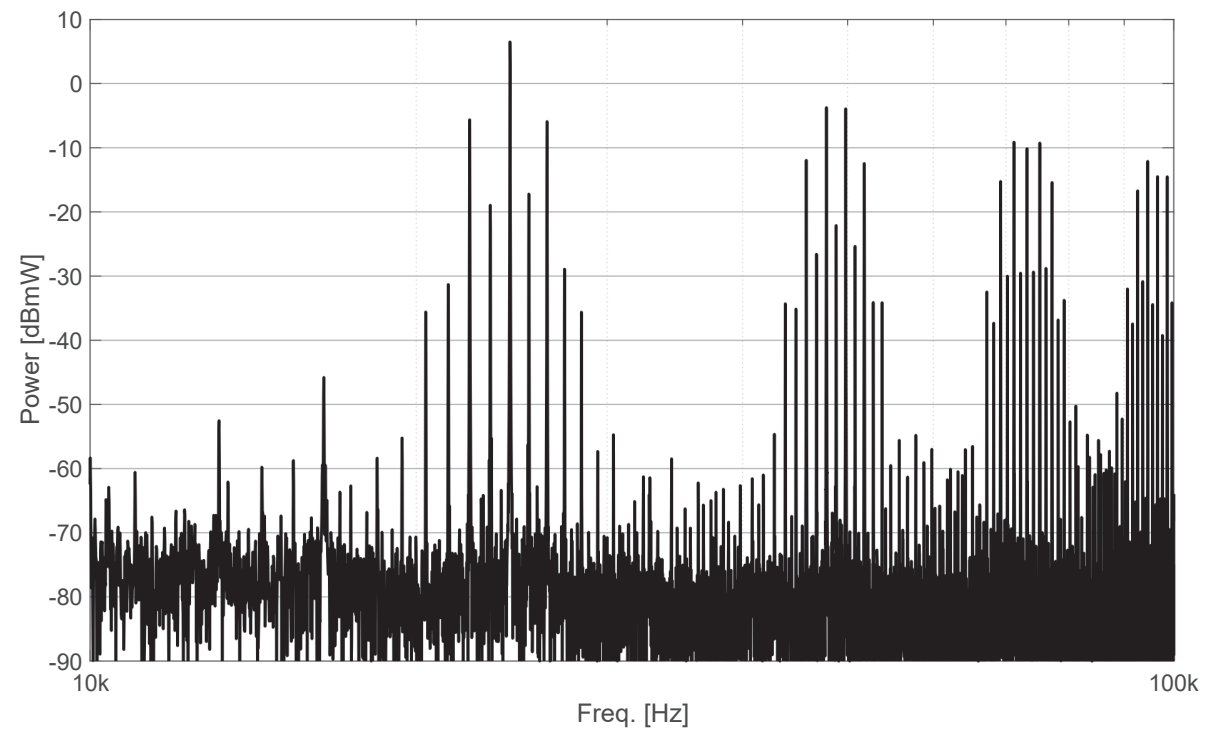

(c) Modulation index of 0.75

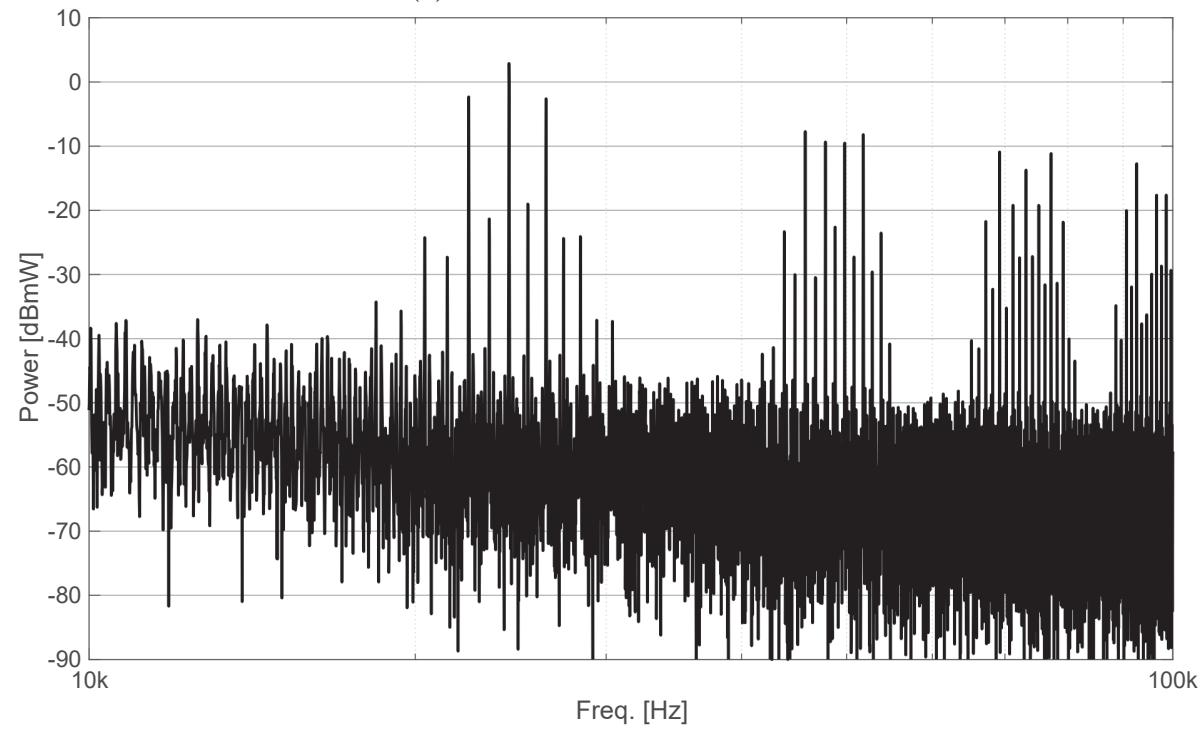

(d) Modulation index of 1

Figure 2.12: Zoomed in versions of the radiative spectra in Figure 2.11. 
The results in Figure 2.11 and Figure 2.12, show similar results as seen in case of the conductive emission results. For higher modulation indexes the subharmonics are increasing, while power in the carrier harmonics is decreasing. Also the sub-harmonics that have significant amount of power are increasing, i.e. the bandwidth of each harmonic carrier is increasing with modulation index. Also the amount of power located at the modulation frequency increases with modulation index, which is related to the increased output AC waveform as can be seen in Figure 2.7.

\subsubsection{Radiated - Magnetic Field Strength}

Next to high switching voltages, the system produces high switching currents which in turn are expected to generate high magnetic fields which can interact with electronic systems nearby. Experience from power converters in motor drive systems learned that these currents are very difficult to predict, and often a trial and error process is followed. Therefore the full wave modeling of the SM as used in the $\mathrm{M} 3 \mathrm{C}$ requires to incorporate magnetic fields as well. The models can eventually be used to analyze and investigate coupling paths. This will assist predictions for possible EMI reductions.

A time efficient magnetic field measurement has been developed in [33] based on COTS Time-Domain Electromagnetic Interference (TDEMI) measurement equipment, which compared to MIL-STD RE101 reduces measurement time from many minutes to mere seconds. DSP has been implemented to mimic the traditional EMI receiver. The magnetic field is measured using a loop antenna, which acts as a transducer for magnetic flux. An output voltage of the antenna is created by means of a varying magnetic flux, which can be seen using the following equations:

$$
\mathcal{E}=-\frac{d \Phi}{d t}, \quad \Phi=\iint_{S} \mu \mathbf{H} \cdot d A
$$

In the measurement setup, Differential Mode (DM) current and magnetic field have both been recorded simultaneously. Allowing an analysis of the relation between transients and magnetic fields in time. However first the measurement setup is described, followed by the results for processing measured fields according to the RE101 standard.

\section{GaN Half-bridge Measurement setup}

In Figure 2.13 it can be seen that the 'DC' source is a galvanic isolated grid that has been rectified. By using sPWM driver logic, the switches are operated in such a way, that the switching node is either connected to the $+165 \mathrm{~V}$ or $-165 \mathrm{~V}$. This results in an sPWM voltage waveform that contains two main frequency components, $f_{c}$ and $f_{m}$, which are the switching frequency and AC output frequency respectively. Similar as the earlier described setups, the FPGA allows for large flexibility in choosing these frequencies. Three unique combinations of $f_{c}$ and $f_{m}$ are processed. 
- $f_{m}=50 \mathrm{~Hz}$ and $f_{c}=25 \mathrm{kHz}$

- $f_{m}=2.3 \mathrm{kHz}$ and $f_{c}=240.4 \mathrm{kHz}$

- $f_{m}=50 \mathrm{~Hz}$ and $f_{c}=80.4 \mathrm{kHz}$

$D_{i}$ and $m_{i}$ are constant in all setups, being 0.5 and 0.35 respectively. This implies that, when the output waveform is low pass filtered, the resulting AC signal consists only of a $f_{m}$ component with an approximate $V_{r m s}=116 \mathrm{~V}$. The load used in this setup was a resistive heater that was designed for $230 \mathrm{~V}$ at $50 \mathrm{~Hz}$. Its impedance was measured with and without attached cable up to $200 \mathrm{kHz}$, the results are included in appendix D and verify that it can be considered to be a broadband resistor. The conducted measurement device is described in Appendix F, and is used to safely measure DM voltages that are being applied to the load.

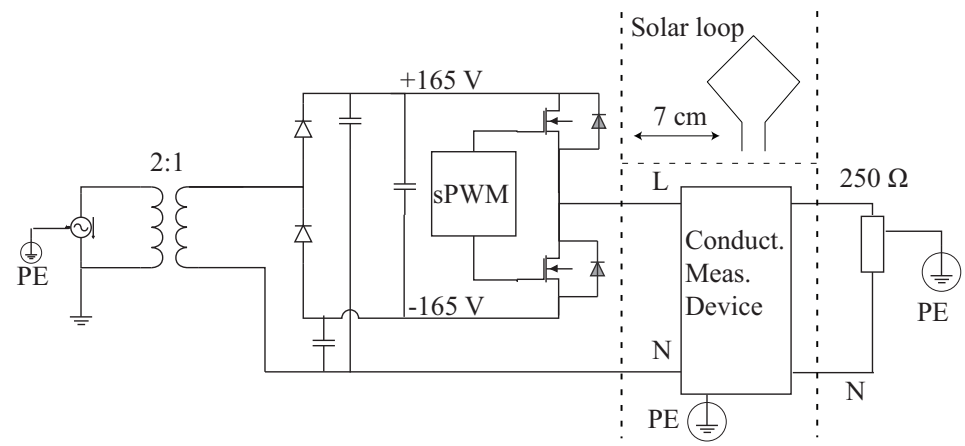

Figure 2.13: Schematic representation of the measurement setup, with the Solar Type 7334-1 Loop Sensor placed near the GaN evaluation board. A detailed description of the conducted measurement device can be found in Appendix F.

\subsubsection{Magnetic Field Strength Results}

The results shown are created through post-processing the recorded time domain signals according to [33]. After which it is corrected with the calibration data of the loop antenna used resulting in a magnetic field in $\mathrm{dBpT}$. Figure 2.14 shows emissions levels below approximately $80 \mathrm{dBpT}$, based on the limits set in the MIL-STD, the results show compliance with all army application even though no EMI mitigation was applied. However, in this case the entire system under test is large and only one position and loop-antenna orientation is considered. It is possible that the loop emitting the radiation is perpendicular to the receiving loop antenna, which reduces measured values considerably. The test performed is therefore not considered to be a full compliance test but a demonstration of the effectiveness of measurement. Figures 2.14a, 2.14b and $2.14 \mathrm{c}$ show high emission peaks at $f_{m}$ and $f_{c}$. Figure $2.14 \mathrm{~b}$ shows that 
the environment in which the measurement was performed is already polluted with $50 \mathrm{~Hz}$ and its harmonics. It was previously shown that the AC current generated in the system at either $50 \mathrm{~Hz}$ and $2.3 \mathrm{kHz}$ should be equal due to similar settings of modulation index and DC-offset. Figures $2.14 \mathrm{~b}$ and $2.14 \mathrm{c}$ both show emission peaks of approximately $123 \mathrm{dBpT}$ at $f_{m}$ which confirm the expectation. Figure 2.14a however shows a difference of approximately $8 \mathrm{~dB}$, it is assumed to be a measurement deviation due to slightly different orientation angle of the loop antenna. Also a reduction of the $50 \mathrm{~Hz}$ harmonics can be seen between in comparing Figure 2.14a with figures $2.14 \mathrm{~b}$ and $2.14 \mathrm{c}$. It was assumed to be the result of the rectifying diodes, which were at first placed close to the GaN half-bridge. This was placed further away in the measurements of figures $2.14 \mathrm{~b}$ and $2.14 \mathrm{c}$, which also slightly altered the orientation of the loop antenna, compare to the measurement of Figure 2.14a. The results presented show that also for magnetic fields, the generated EMI can be tuned by choosing different combinations of modulation and carrier frequency. Further development of the measurement technique will allow a more accurate assessment of highly radiative or suppressed frequencies in the system under test, first steps where taken in this endeavor in $[34,35]$.

\subsection{Summary}

This chapter has been dedicated to describing and evaluating a single halfbridge submodule that can be used in the final multi-level converter. As the $\mathrm{SM}$ is the basic building block of setup, it is necessary to understand its impact as a noise source. At the beginning of the chapter a basic theoretical model for EMI estimation was given. The model has been compared to measurements, which verify the EMI generated is directly related to sPWM control signals.

Conducted and radiated measurements were performed considering the relative low frequency range between $2 \mathrm{kHz}$ and $150 \mathrm{kHz}$, as this has become a significant research interest. At the moment of writing this thesis, no civil standards exist in this frequency range, but EMI generated here has been identified as the culprit in a rising number of EMC related instances. As the M3C is considered to be a possible cornerstone of the future Smart grid, compatibility in this frequency range is required even before standards exist. As susceptibility issues are to be expected in existing equipment. This chapters contribution thus has been a simple noise source model can be used to predict the magnitude as well as the frequencies at which EMI is being generated. In later chapters of the thesis it will be shown that it can also be used to predict possible mitigation techniques. However in the next chapter a multi-level FPGA controller is developed, and the control parameters are evaluated in their influence on the generated EMI. As the current chapter has shown the direct relation between sPWM control signal and the generated EMI, a study is required into the control parameters in a multi-level case which is basically the series connection between a multitude of already identified noise sources. 


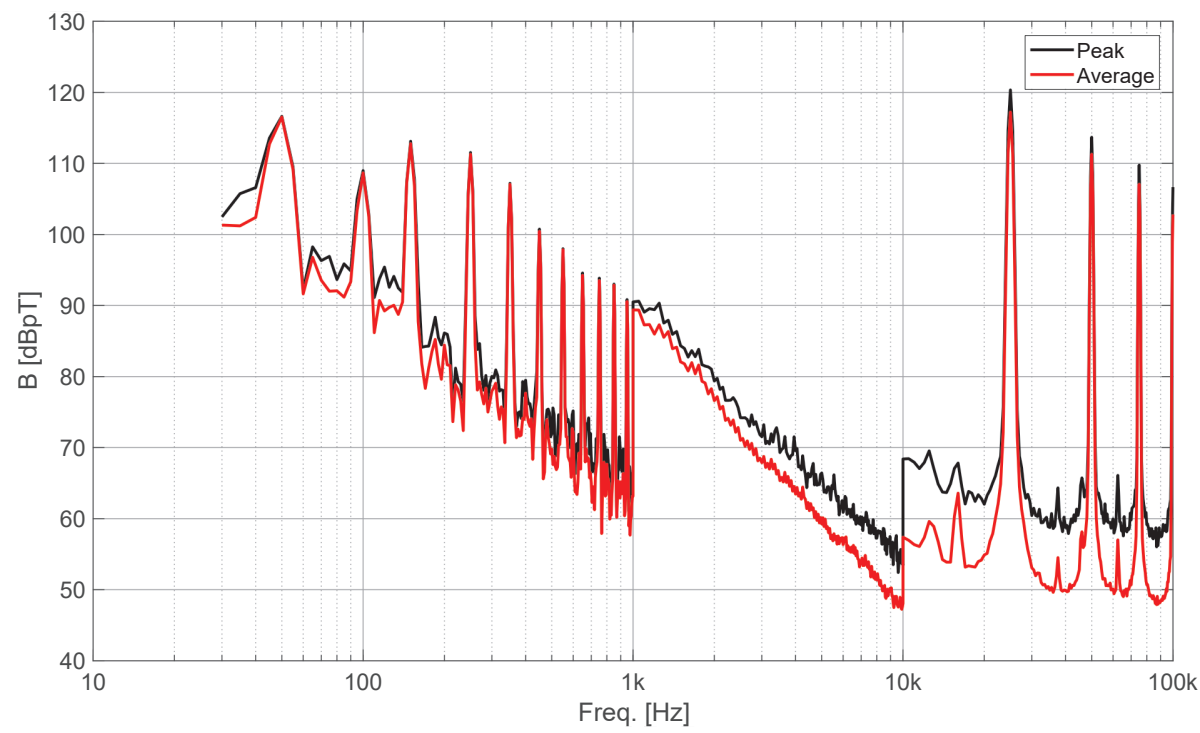

(a) Results for $f_{c}=25 \mathrm{kHz}$ and $f_{m}=50 \mathrm{~Hz}$
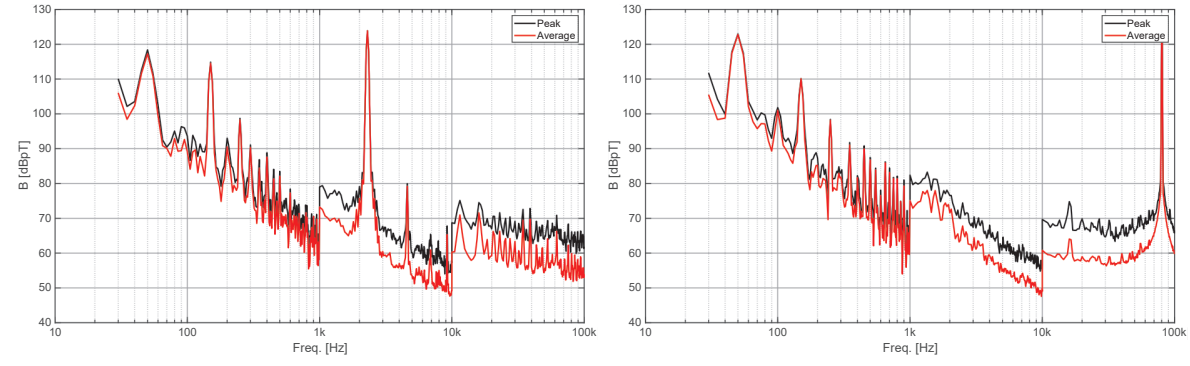

(b) Results $f_{c}=240.4 \mathrm{kHz}$ and $f_{m}=(\mathrm{c})$ Results for $f_{c}=80.4 \mathrm{kHz}$ and $f_{m}=$ $2.3 \mathrm{kHz}$ $50 \mathrm{~Hz}$

Figure 2.14: Measured magnetic radiation spectra processed according to RE101. Red shows peak values while black is showing average values over time. 


\section{Chapter 3}

\section{sPWM in M3C}

The introduction of this thesis has described the envisioned multi-level converter system. Investigations in the generated EMI remain difficult, due to the complexity of the total system and the dependency on (often unpredictable) parasitics [11-13]. Previous chapter has described a single SM as a noise source inside the system, however the interaction between multiple noise sources within such a system are highly dependent on the integration and implementation of the system. Correlation (i.e. non-independence) between SMs exists, as they work together to determine the functional behavior of the converter. The origin of the correlation is in the control system. Hence this chapter starts with the development of a flexible signal generator capable of controlling an $8 \mathrm{SM}$ converter. The EMI generated in the system is directly related to the behavior of the signal generator, therefore an elaborate evaluation of performance has been done. Further a verification has been performed by implementing the generator inside multiple systems. First in a DC/AC converter, as the behavior is well known and directly comparable with results shown in the previous chapter. Next the generator has been implemented in a simplified multi-level implementation, that represents the subsystem of the M3C. Two SMs were interconnected with the assumption their capacitors are charged and capable of providing power, which is based on the functional behavior described in the introduction. The secondary power loop as implemented in the M3C uses a frequency different from the output frequency to keep the voltage balanced over each SM. After the verification of its functional behavior, a study is performed on the generated EMI in the system.

The first part of this chapter is part of a manuscript under revision and to be submitted, while the second part of this chapter has been published in [36] 


\section{1 sPWM Generator Design}

Several challenges were identified in the development of M3C, most notable are optimal control strategy [7-9], potential EMI issues, and module interactions [3]. It is well known that the control scheme is directly related to the amount of EMI being generated $[16,17]$, and many papers describe methods for reducing EMI inherently [20-22] or by optimizing the usage of parasitics [23].

A trend in switching controls for inverters and converters has been the development of FPGA based control [37,38]. FPGAs have been found highly suitable for control for a multitude of reasons, like parallelism, speed, reprogram ability, and possible feedback schemes [39].

This section focuses on the development of an FPGA based sPWM generator for high switching frequency, multi-level converters possibly implementing GaN and Silicon-Carbide ( $\mathrm{SiC}$ ) based switches. A low modulation frequency $\left(f_{m}\right.$ i.e. the output frequency) is often chosen in DC/AC converter topologies (i.e. $50 / 60 \mathrm{~Hz}$ ), however in the M3C topology of [10], one is limited by approximately $f_{c}>10 f_{m}$. With $f_{c}$ being the switching frequency of the individual semiconductors. DC/AC converters typically operate at switching frequencies ranging between $1 \mathrm{kHz}$ to $100 \mathrm{kHz}$. GaN and $\mathrm{SiC}$ technologies enable even higher switching frequencies [40]. M. Lakka et al. has shown in [41] that FPGAs are suitable to achieve switching in the order of $1 \mathrm{MHz}$, while [42] showed this is also possible using a Digital Signal Processor (DSP). Benefits of DSPs are often lower cost and also capable of being an Analog-to-Digital Converter (ADC) and Digital-to-Analog Converter (DAC). J. Liu et al. also showed the ability to produce higher frequency $\mathrm{AC}$ voltages of $1 \mathrm{kHz}$, this however often requires a modified sampling method [19]. Also due to the high switching frequency required, the functionality of the microprocessor might be limited. FPGAs allow concurrent operation of control algorithms, which allows scalability. FPGA based sPWM generators for multilevel converters have been shown in for instance $[43,44]$, and often require an understanding of a Hardware Description Language (HDL). The method presented here, solves this issue by using automated code generation algorithms available in Simulink.

The flexible sPWM generator is required to study the EMI generated (and possible mitigation methods) in an $\mathrm{M} 3 \mathrm{C}$ system. The generator is created with adjustable parameters related to the application shown in [10] and described in the introduction of this thesis. The demonstrator of the proposed topology by [5] was developed and the feasibility and modularity were demonstrated (Figure 1.4). Based on the developed demonstrator, the following control parameters are identified, which are directly related to the design challenges/requirements of the developed generator:

1. Carrier Frequency $(10 \mathrm{kHz}-2 \mathrm{MHz})$

2. Modulation Frequency $(0 \mathrm{~Hz}-250 \mathrm{kHz})$

3. AC-amplitude $(0.0-0.5)$ 
4. DC-offset $(0.0-1.0)$

5. Dead-time ( $0-630 \mathrm{~ns})$

6. Number of levels (1, 2 or 4$)$

The novelty of the proposed development lies in its flexibility in input parameters, which allows the generator to be tuned to a wide range of applications [45-48]. From low to high switching frequencies and low to high AC frequency applications, like induction heating or wireless power transfer. The architecture is also not limited to a single type of power converters. It can be used in half and full bridge typologies, as well as multi-level or multi-phase systems. The system is developed to be dependent on the internal clock frequency, increasing possible switching speeds and output frequencies is as simple as interchanging the FPGA development board by one that runs on a higher internal clock frequency. The spectral pureness of the signals is dependent on the clock frequency. Thus increasing switching frequencies while the clock frequency is constant will result in larger errors. A large benefit of the MATLAB implemented design, is that a simple for-loop allows for a highly time efficient investigations with respect to the controllable parameters (e.g. efficiency dependence on switching frequency).

In section 3.1.1 a description of the developed generator is given. The subsections are created based on the architecture and control parameters of the developed sPWM generator. In section 3.1.2 the implementation of the architecture and its evaluation are discussed through measurements at the board level. Its applicability is shown in section 3.1 .3 by applying the generator to a GaN DC/AC-converter and a simplified SiC multi-level converter.

\subsection{1 sPWM Generator}

Automated generation of VHSIC Hardware Description Language (VHDL) code was used to program the FPGA. I.e. the sPWM generator for multilevel converters was designed without writing a single line of code. This enables engineers to investigate the M3C (or any other converter with an sPWM control scheme) operation without knowledge of any HDL. The control strategy used is Phase Shifted sinusoidal Pulse Width Modulation (PS-sPWM), which means the reference waves are shifted for every SM (Figure 2.2e). The comparator runs on the system clock, which allows for scalability in frequency when FPGAs are used with higher internal clocks. After the comparator, a variable dead-time is inserted. Dead-time is required to prevent shorting faults. The architecture used can be seen in Figure 3.1. This section is divided according to these three sub-controlsystem stages. Future development of the generator can be done by adding subsystems. 


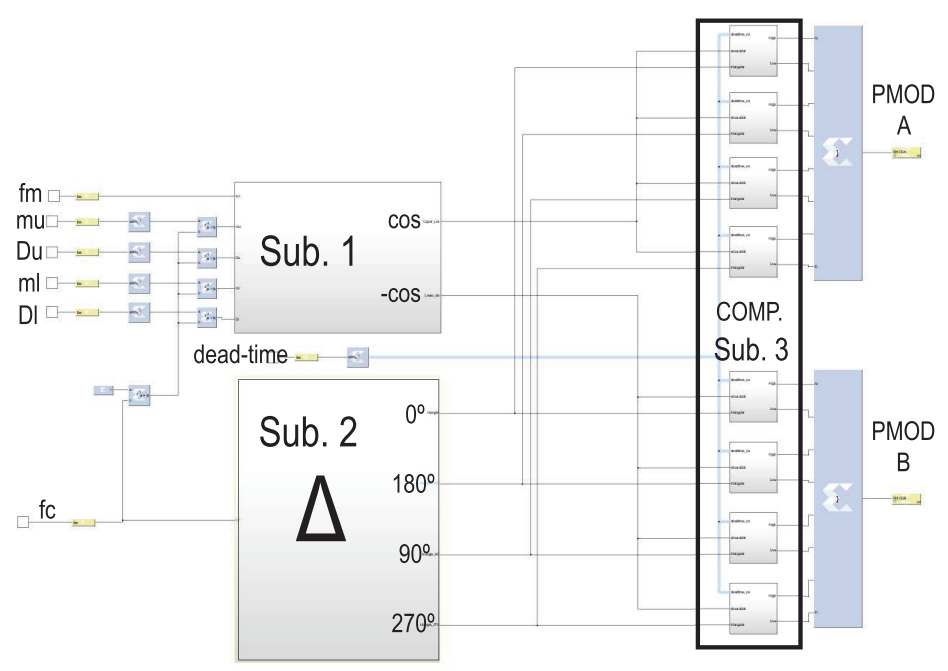

Figure 3.1: 1. sinewave generator, 2. triangle-wave generator, 3. comparators.

\section{Sine-wave Generator}

The sinusoidal waveform is used for modulating a PWM and hence its frequency is dubbed the modulation frequency $\left(f_{m}\right)$. The sinusoidal waveform is produced using "DDS compiler" and "CORDIC". Which are a Direct Digital Synthesizer and COordinate Rotational DIgital Computer algorithm respectively. In this case, the DDS compiler produces a clock dependent phase and the CORDIC core translates this into a sine wave. The DDS compiler has a sin/cos LookUp Table (LUT) however the output frequency becomes non-programmable, creating a less flexible system. The DDS is therefore used as a phase generator only, which output is controlled by a phase increment value $\Delta \Theta$. The frequency resolution of the synthesizer is a function of the clock frequency and can be determined by:

$$
\Delta f=\frac{f_{c l k}}{2^{B_{\Theta}(n)}}
$$

With $B_{\Theta}(n)$ being the number of levels determined by the number of bits $n$ in the phase accumulator. High flexibility of the output frequency is wanted and requires a certain spectral purity. The output frequency is defined as:

$$
f_{m}=\Delta f \cdot \Delta \Theta
$$

Combining Equations 3.1 and 3.2 shows that for a $100 \mathrm{MHz}$ clock frequency, one requires at least 27 bits to attain a frequency resolution of $1 \mathrm{~Hz}$. The phase output ranges from $-\pi$ to $\pi$ that is translated by the CORDIC ip-core to sine (and cosine) functions that can be scaled and offset. In case of the M3C, the outputs of the sinewave generator are synchronous sine and cosine waveforms of similar frequency. These can independently be scaled and independently an 
offset can be added. Which can be seen in the following equation:

$$
x(t)=m_{i} \cdot \sin \left(2 \pi \cdot f_{m} \cdot t\right)+D_{i}
$$

With $i$ being either $u$ or $l$ depending on whether the SM is part of the upper or lower arm respectively. The following five control inputs are then identified:

1. modulation frequency: $f_{m}$

2. AC-amplitude: $m_{u}, m_{l}$

3. DC-offset: $D_{u}, D_{l}$

The inputs are normalized to the triangular wave amplitude, as can be seen in Figure 3.2 with the exception of the desired modulation frequency.

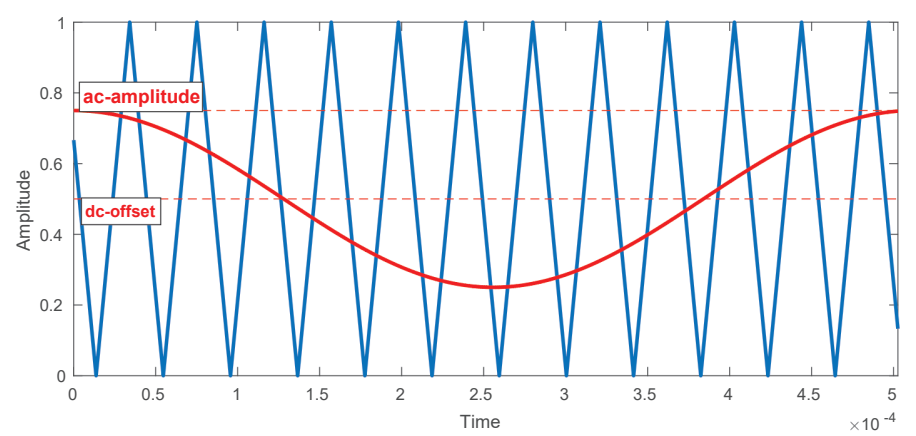

Figure 3.2: Amplitudes normalized to max of the up and down counter $\left(N_{c n t}\right)$.

\section{Triangle-wave Generator}

In case of Multilevel Converters (MC), comparing the phase voltage reference waveform with carriers produces an SPWM, with transitions corresponding to inserting or bypassing submodules. S. Debnath et al. discusses carrier schemes with a phase rotation scheme as a possible solution to equally distribute the voltage ripple across the SMs [9]. In the developed architecture this phase rotation scheme was implemented through the creation of four phases $\phi=$ $[0,90,180$ and $270 \mathrm{deg}]$. The basic structure consists of a single up/downcounter that is the reference waveform $\phi=0$. Two techniques are implemented to create the different phases from this reference signal. The $\phi=180$ wave is created by inverting (i.e. multiplied with -1) and offsetting the reference wave. The $\phi=90$ and 270 are created by interchangeably selecting and offsetting previously generated waveforms.

With the continuously output of four phases, one is able to create a converter with 1,2 , or 4 levels. The switching frequency is determined by the counter 
and related to the clock frequency of the FPGA:

$$
f_{c}=\frac{f_{c l k}}{2 \cdot N_{c n t}}
$$

Where $N_{c n t}$ is the highest integer value it counts up to. The resulting frequency is halved, due to it being an up and down counter.

\section{Dead-time Inserter}

The basic functionality of the dead-time inserter is based on delaying a rising edge in the PWM signal. The delay is controllable by setting an integer value. The input signals can be seen in Fig 3.3, which are each others inverted outputs of the comparator. As the PWM is high, a counter starts to run which is compared to the set integer at every clock instance (Figure3.4a). When the counter is equal or exceeds the set value, the relational statement produces a high output. Only when the original PWM signal and the output of the relational statement are high, the output is high. Effectively delaying the rising edge with a set number of clock counts (Figure3.4b). Note that dead-time is specifically required in half-bridges since they are DC-fault prone [9].

The sPWM generator is specifically developed for the project described in $[3,6,10,22]$. The architecture was therefore implemented on an FPGA with a clock frequency of $100 \mathrm{MHz}$, as it would be powerful enough to create a sPWM signal based on a $f_{c}=24.4 \mathrm{kHz}$ and $f_{m}=1.983 \mathrm{kHz}$. The next section will discuss the implementation on an Xilinx FPGA development board. In short, the architecture is designed with Simulink and compiled to a binary file. For simplicity of control, the binary file is also uploaded via Simulink to the board, together with the controllable parameters. These can be set either from MATLAB in the current workspace, or set in the Simulink simulation environment.

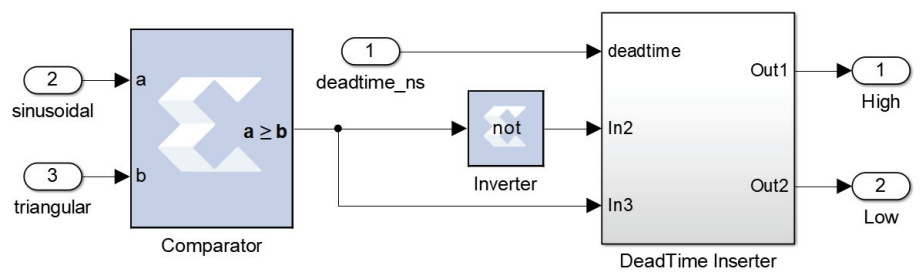

Figure 3.3: Comparator and dead-time insertion subsystem.

\subsubsection{Implementation}

The previous section has addressed the method of creating the desired sPWM signals based on parameters that are used in a specific architecture for MCs. 


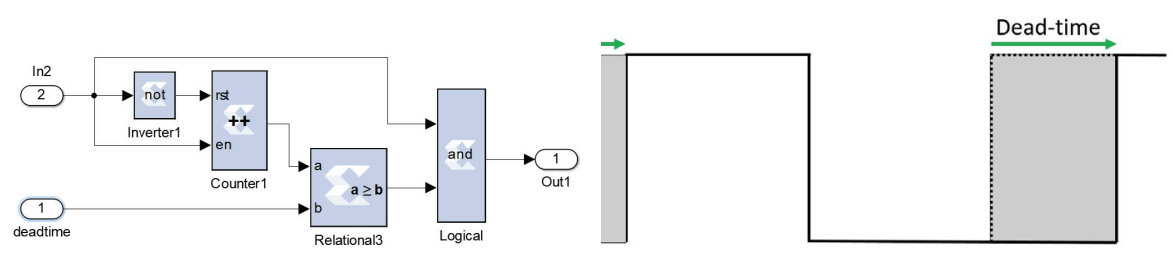

(a) Rising edge delay system

(b) Delayed rising edge

Figure 3.4: Rising edge dead-time insertion.

The following section is focused on the realization of the system. As an FPGAevaluation board is chosen, certain limits are imposed. Amount of Memory, DSP slices, LUTs, clock frequency and rise/fall times of the I/O ports all have an influence. In this section a general characterization of the setup is given, based on parameters that are controllable. These are restated here: $f_{c}, f_{m}$, $m_{i}, D_{i}, t_{\text {dead }}$ and number of levels.

\section{Digilent}

The Digilent Nexys3 evaluation board is used in this setup. The FPGA evaluation board contains a Xilinx Spartan 6 FPGA running on a $100 \mathrm{MHz}$ clock. As was demonstrated in [41], the approximated highest possible PWM waveform switching frequency is about $1 \mathrm{MHz}$. The developed architecture is completely dependent on the internal clock frequency. Using a development board (e.g. Nexys4 at $450 \mathrm{MHz}$ ) with a higher clock, will increase the maximum achievable switching frequencies and power frequency. The processing chain can be seen in Figure 3.1. $f_{m}$ and $f_{c}$ are set in MATLAB and implemented in Simulink. A auto-generated bitstream is uploaded to the board. The device resource utilization is independent of the control parameters, carrier-, clock- and modulation-frequencies. The required FPGA resources are stated in Table 3.1. The architecture has not been optimized, however the required resources are comparable to [41] and occupy a small fraction of medium-sized FPGAs.

The resulting digital signals are routed to a set of I/O pins that are located within a Digilent specific Peripheral Modules (PMOD) connector. The board contains four identical PMOD connectors designated A to D, shown in Figure 3.5. It contains two pins with $3.3 \mathrm{~V}$, two grounding pins and eight signal outputs that will individually control the switches.

\section{\# of channels}

The sPWM generator has 32 outputs and is therefore suitable for a multi-level converter. Depending on the submodule topology one could design a system with 1 to 8 levels. In the prototype version of sPWM generator 16 outputs are simultaneously switched, therefore 8 submodules can be controlled in sync, which results in 4 levels. They are controlled based on the control parameters 
Table 3.1: Device Utilization.

\begin{tabular}{|l||c|c|}
\hline & Absolute & To max. \\
\hline DSP's & 14 & $43 \%$ \\
\hline Slice Registers & 2,278 & $12 \%$ \\
\hline Slice LUT & 2,825 & $31 \%$ \\
\hline BRAMs (16k/8k) & $2 / 9$ & $6 \% / 14 \%$ \\
\hline
\end{tabular}

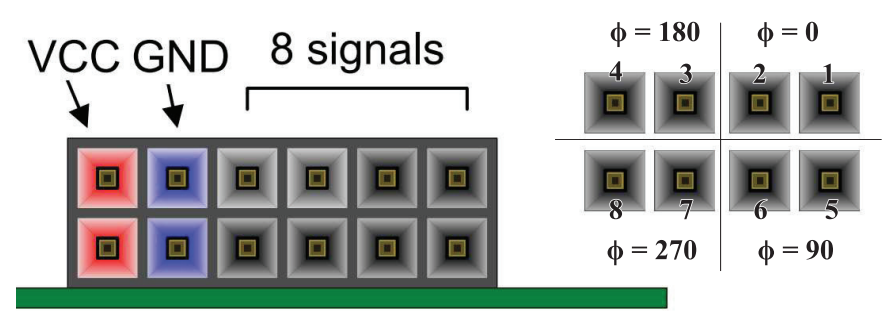

Figure 3.5: PMOD Input/Output.

that are available in [10]. Depending on the settings, the 16 ports all have individual PWM outputs. To determine the performance of simultaneously switching $16 \mathrm{I} / \mathrm{O}$ ports, the rise and fall times were individually measured with a Keysight DSO-X 3024A oscilloscope with 4 GSa/s and averaged over 512 samples. The generator was set to a switching frequency of $100 \mathrm{kHz}$ with a $50 \%$ duty-cycle. The trigger was set to $1.65 \mathrm{~V}$ at which is $t=0$ in Figure 3.6. Rise and fall times are calculated with $90 / 10 \%$ values. Based on the resulting waveforms, we can consider the FPGA to be suitable for MCs containing 16 switches. Which in case of the studied M3C and PS-sPWM results in 5 voltage levels.

\section{Phase Error}

To accommodate the 4 SM MCs, the triangular waves should be phase shifted equally resulting in four phase shifted PWM signals as seen in Figure 3.7. A phase shifted carrier is a delayed version of the one considered to be the 'original' triangular wave. The system is digital and delays can only be implemented in integer clock-cycles. Therefore the period (in clock-cycles) should be dividable by the number of phases produced. This results in a limited set of possible carrier frequencies determined by:

$$
f_{c}=\frac{f_{c l k}}{2 N_{c n t}} \quad \text { with } \frac{N_{c n t}}{N_{\phi}}=\mathbb{Z}_{\leq 0}
$$

With $N_{\phi}$ being the number of phases, which in our case is four. Therefore the counter should always count up to multiples of 4 . In case of using a $N_{c n t}$ 

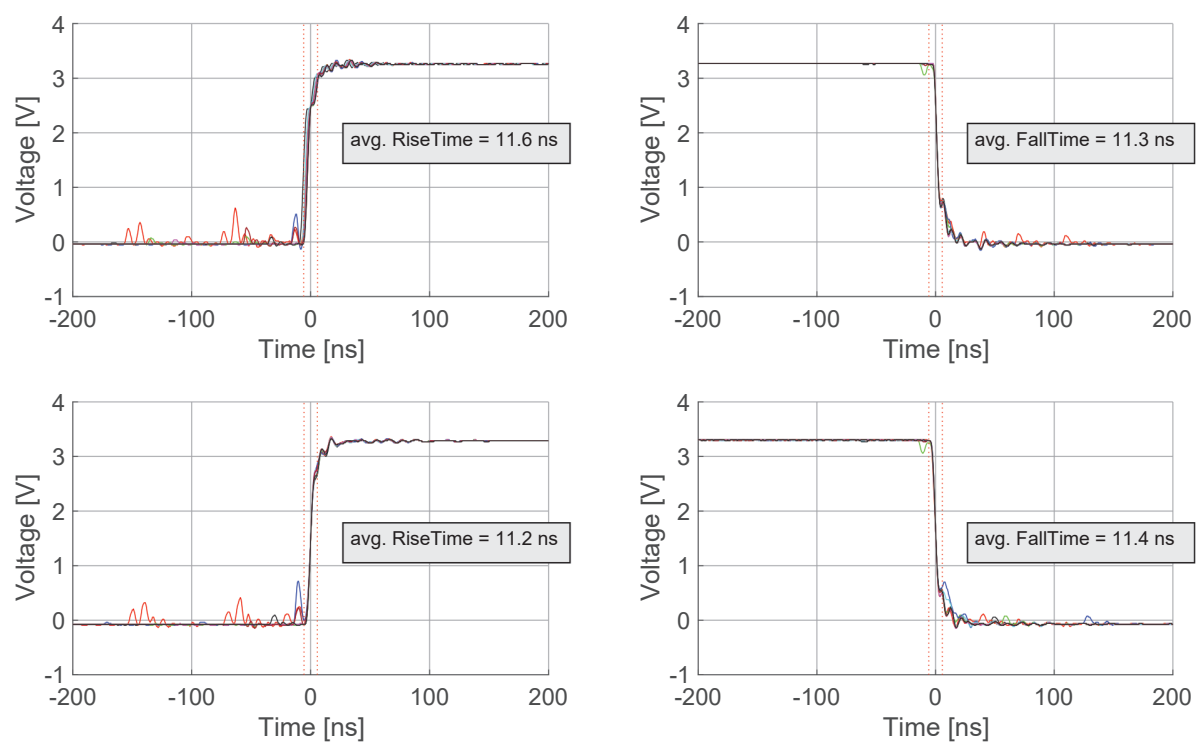

Figure 3.6: PMOD performance with PMOD A and B respectively upper and lower plots.

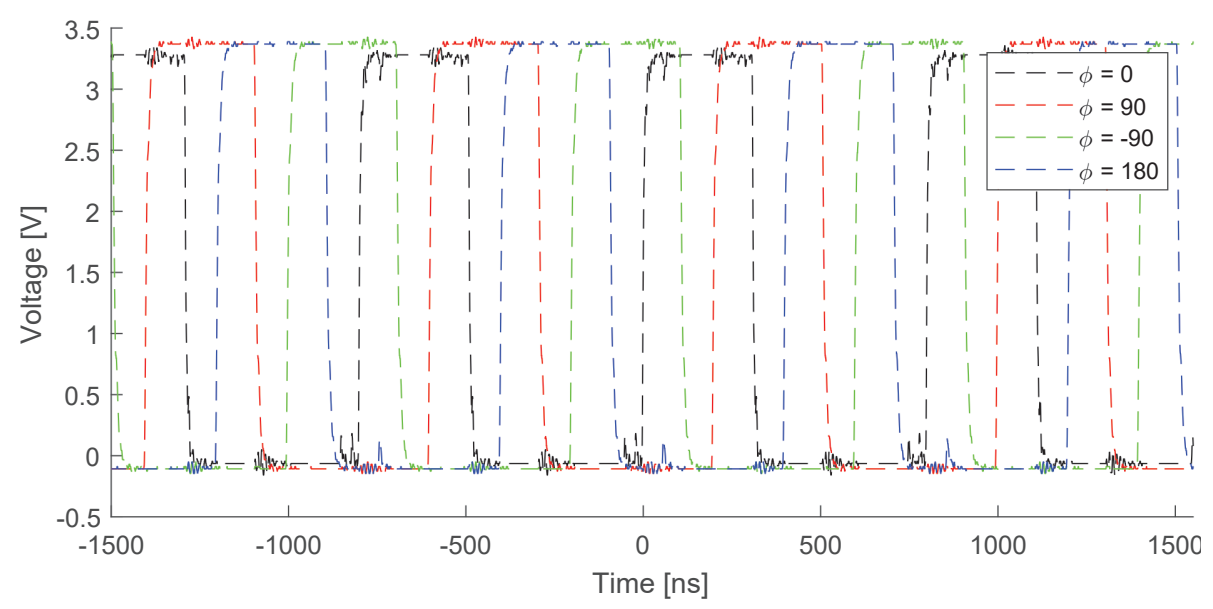

Figure 3.7: PMODA all four phases measured at $40 \%$ duty-cycle $1.25 \mathrm{MHz}$, measured at port 1, 3, 5 and 7 from Figure 3.5. 
that is not dividable by the number of phases, one will introduce a phase error which is compared in Table 3.2 for $N_{c n t}=39,40$ and 41 . However this error decreases for lower carrier frequencies.

Table 3.2: Measured phase.

\begin{tabular}{|c||c|c|c|}
\hline$\Delta \phi$ & $N_{c n t}=39$ & $N_{c n t}=40$ & $N_{c n t}=41$ \\
\hline $1 \rightarrow 2$ & $91.6^{\circ}$ & $89.3^{\circ}$ & $86.9^{\circ}$ \\
\hline $1 \rightarrow 3$ & $-88.4^{\circ}$ & $-90.7^{\circ}$ & $-93.1^{\circ}$ \\
\hline $1 \rightarrow 4$ & $179.8^{\circ}$ & $179.8^{\circ}$ & $179.6^{\circ}$ \\
\hline
\end{tabular}

\section{Maximum switching frequency}

To determine the highest possible switching frequency a sweep was performed by increasing the carrier frequency. In this case, the amplitude of the sinewave was set to 0 with a DC-offset of 0.5 . Resulting in PWM signals with a $50 \%$ duty-cycle. As the carrier frequency is set by an up and down counter, the maximum frequency would be at $N_{c n t}=1$, resulting in $50 \mathrm{MHz}$. In this extreme case, no dead-time can be inserted and only the $50 \%$ duty-cycle can obtained. In case of $N_{c n t}=4, f_{c}=12.5 \mathrm{MHz}$. This is assumed/considered to be the maximum achievable $f_{c}$ while maintaining the possibility to introduce dead-time and still have a duty-cycle, as it consists of 8 clock-periods and 5 integer levels.

\section{Duty-Cycle Error}

Based on the measurement sweep done, the deviation of set duty-cycle increases with switching frequency. In case of the $f_{c, \max }=12.5 \mathrm{MHz}$ this results in a $23.5 \%$ deviation over the high and low switches, as is shown in Figure 3.8. The pulse-period is set to $80 \mathrm{~ns}$, therefore the deviation is approximately $9.4 \mathrm{~ns}$ in each switch. Intuitively this can be explained by the fact that the I/O pins have a fall and rise time of approximately 11ns. Every period an error of approximately $10 \mathrm{~ns}$ is being made, which can become significant when reducing the period time, as the number of rising and falling edges increase.

\section{Quantization Error}

Anytime a digital system is representing a continuous waveform a 'quantization error' is apparent. The integer values of the counter can be seen as quantization levels, by setting a threshold for the comparator a decision error is made in the transition across the threshold. The duration of this error is depended on the clock time and the decimation/remainder of the threshold.

$$
t_{\text {error }}=(1-\alpha) t_{c l k}
$$


With $\alpha$ being the value behind the decimation point. However this should only cause a delay in the waveform. Based on the assumption that a $5 \%$ deviation (over both switches) is considered acceptable, the highest possible switching frequency is determined to be $2.6 \mathrm{MHz}$.

A more interesting phenomena appears when the threshold is set to an integer value. In this case, there is a moment per period that the carrier wave equals the threshold voltage. Due to the comparator being complementary either with $\leq$ and $>$ or $\geq$ and $<$, the occurrence of an equal valued threshold favors a state. For the assumed maximum carrier an easily envisioned example shows that for $N_{c n t}=4$, at $50 \%$ duty-cycle results in the same waveform as $60 \%$ duty-cycle. The waveforms recorded with a setting of $50 \%$ duty-cycle can be seen in Figure 3.8, which by post-processing show to be nearly identical to a $60 \%$ duty-cycle.

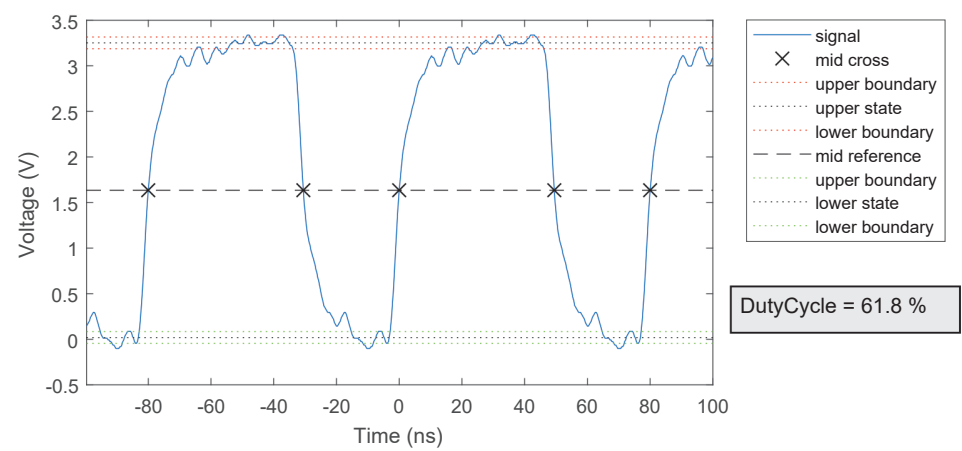

(a) High switch PWM signal

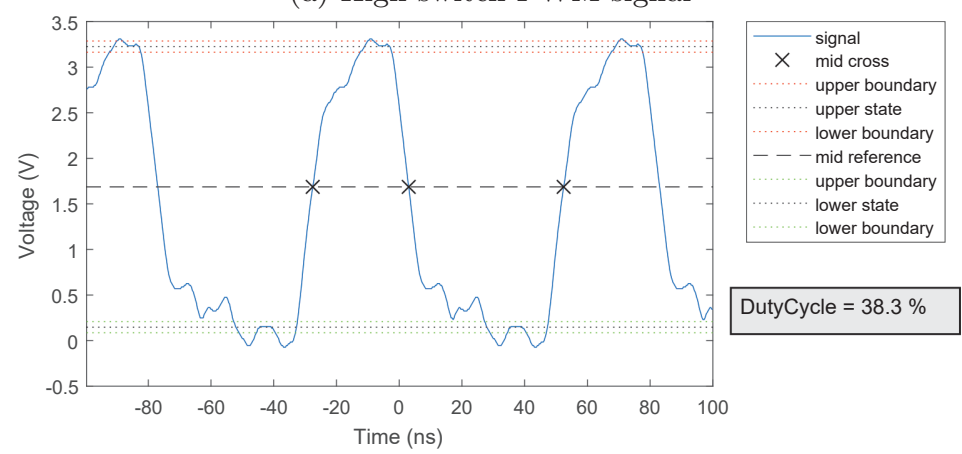

(b) Low switch PWM signal

Figure 3.8: The measured duty-cycles for high and low at $12.5 \mathrm{MHz}$ switching frequency with set duty-cycle to $50 \%$. 


\section{Modulation Frequency}

In the previous sections the generation of a standard PWM signal with a static duty-cycle has been discussed and evaluated. However considering the generator to be used as the control logic in any (multilevel) converter, this PWM can be sinusoidally modulated. This is particularly useful in DC/AC conversion, with the modulating frequency being the resulting sinusoidal wave at the output. In the currently available power systems, this AC signal is of extremely low frequency $(16.6 \mathrm{~Hz}$ to $1 \mathrm{kHz})$ compared to the possible switching frequency. In the developed system, one is only limited by the set switching frequency and by general rule of thumb the following relation holds [10]: $f_{c}>10 f_{m}$

In case of a half bridge based submodule the signals of the pin pairs responsible for a single phase, shown in Figure 3.5, should be inverse. For the evaluation of the implemented architecture, the generator was set to $f_{c}=1.25 \mathrm{MHz}$, $f_{m}=80 \mathrm{kHz}, D_{i}=0.4, m_{i}=0.25$ with zero dead-time inserted. In Figure 3.9 the measurement result is displayed. Since the switching transitions are difficult to distinguish, an ideal lowpass filtered version of the SPWM is displayed on top of the waveform. This shows the generator is capable of producing control signals for DC/AC converters with high frequency alternating current.

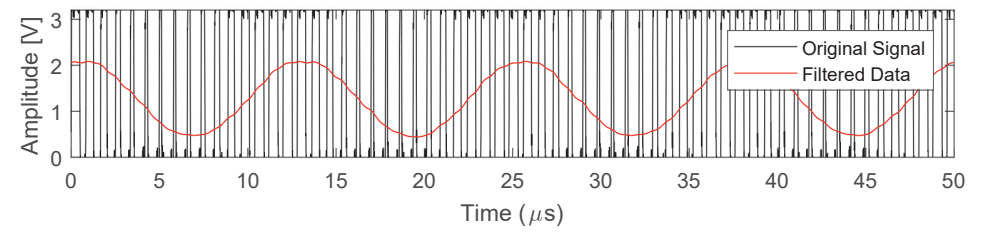

(a) Pin 1

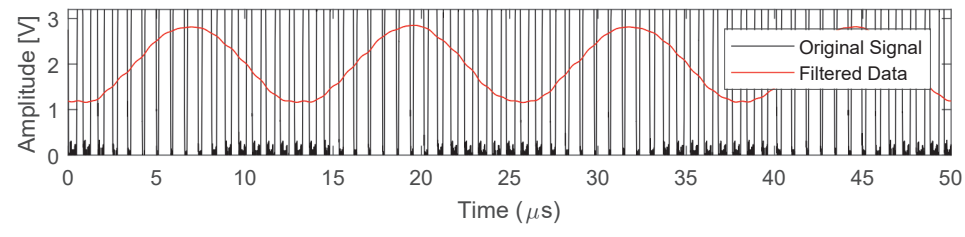

(b) Pin 2

Figure 3.9: The frequency modulated output of pin 1 and 2 (Figure 3.5) with a lowpass filtered version at $f_{c}=1.25 \mathrm{MHz}$ and $f_{m}=80 \mathrm{kHz}$.

\section{Dead-time insertion}

The dead-time insertion mechanism was discussed in section 3.1.1. Apart from the fact that it will have an influence on the perceived duty-cycle, carrier and modulation frequency, here only a verification of its implementation is given. In Figure 3.10 the sPWM waveforms with similar settings that were used in the frequency modulation verification section can be seen. $f_{c}=1.25 \mathrm{MHz}$, 
$f_{m}=80 \mathrm{kHz}, D_{i}=0.4, m_{i}=0.25$. In the upper and lower figure, one can see the result of inserting respectively $50 \mathrm{~ns}$ and $100 \mathrm{~ns}$ of dead-time. It shows that the space between rising and falling edges increases for larger inserted dead-times, thus both pins are longer in a low state together.

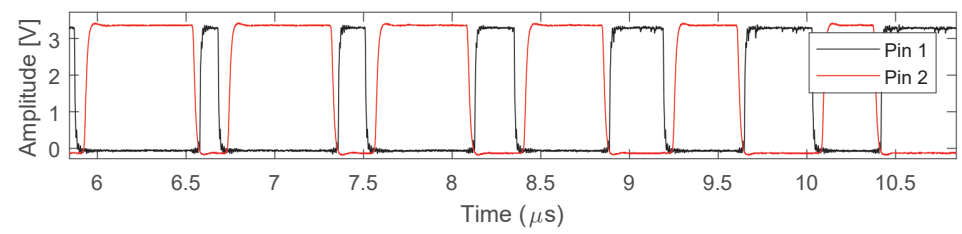

(a) Dead-time $50 \mathrm{~ns}$

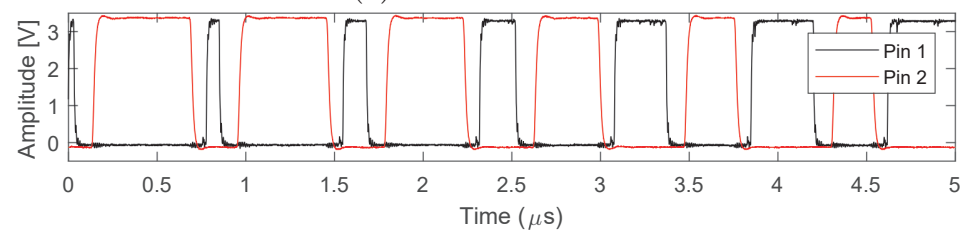

(b) Dead-time $100 \mathrm{~ns}$

Figure 3.10: The frequency modulated output of pin 1 and 2 (Figure 3.5) with different dead-times at $f_{c}=1.25 \mathrm{MHz}$ and $f_{m}=80 \mathrm{kHz}$.

This section has shown the possibility to generate high carrier and modulation frequency sPWM signals for multi-level converters using a MATLAB/Simulink controllable FPGA. It has been verified using multiple test setups. In the next section first a GaN based DC/AC converter is controlled using this generator and later a $\mathrm{SiC}$ based multi-level converter. Which provides a validation of the usability of such a powerful and flexible sPWM generator.

\subsubsection{Evaluation}

In this section two evaluation setups of the designed sPWM generator are discussed, to verify the applicability in conventional DC/AC type of converters and in the desirable multi-level converters.

\section{DC/AC converter}

Using different carrier and modulation frequencies, power is shifted to many different frequencies. Verification measurements have been performed by measuring the output power of the converter while a broadband linear load is applied. A Yokogawa WT500 power analyzer is used while applying three in-line lowpass filters in parallel. Figure 3.11 is a schematic representation of the measurement setup. The filters that are applied have cut-off frequencies at $500 \mathrm{~Hz}$, $5.5 \mathrm{kHz}$ and $100 \mathrm{kHz}$. The DC power supply used was set to $\pm 10 \mathrm{~V}$. The mea- 
surements have been performed for a high number of different configurations within the following parameters:

1. Carrier Frequency: $50 \mathrm{kHz}-800 \mathrm{kHz}$

2. Modulation Frequency: $50 \mathrm{~Hz}-50 \mathrm{kHz}$

3. AC-amplitude/Modulation index: $0.25 / 0.5$

4. DC-offset: 0.5

5. Dead-time: $100 \mathrm{~ns}-200 \mathrm{~ns}$

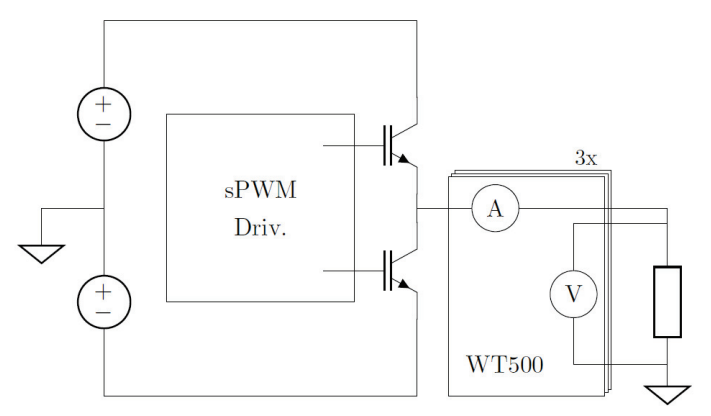

Figure 3.11: DC/AC converter setup using a GaN based COTS half-bridge used to verify the designed sPWM driver.

Figure 3.12 and Figure 3.13 are displaying a subset of these results with every marker representing a frequency measurement point. In Figure 3.12 the three parallel lowpass filters (LP) can be seen in different colored lines. Black, red and green are respectively $500 \mathrm{~Hz}, 5.5 \mathrm{kHz}$ and $100 \mathrm{kHz}$. The spectral content of an sPWM signal has been shown in for instance [22]. Thus the power is also concentrated around $f_{m}$ and $f_{c}$ and all of their harmonics and subharmonics. In Figure 3.12, the green line depicts the situation that measures the power up to approx. $100 \mathrm{kHz}$. As $f_{m}$ and $f_{c}$ are below the filter's cutoff frequency it shows the total dissipated power. While the other lines exclude a part of the power as $f_{c}$ is larger then their cutoff frequencies. Thus the large difference in recorded output power is due to the carrier frequency being below the filter's cutoff frequency. By increasing $f_{m}$, the power located at $f_{m}$ will be excluded, as $f_{m}$ surpasses the cutoff frequencies. The results show the output power to be (almost) independent of generated $\mathrm{AC}$ frequency, confirming the broadband characteristic of the load. The effect of the applied inline filters can also be clearly seen. The black line shows a $-3 \mathrm{~dB}$ at $500 \mathrm{~Hz}$, while the green line shows it at $5.5 \mathrm{kHz}$, as expected.

In Figure 3.13 the three colored lines are representing different implemented dead-times. Results presented in this figure are for a single LP filter only, 


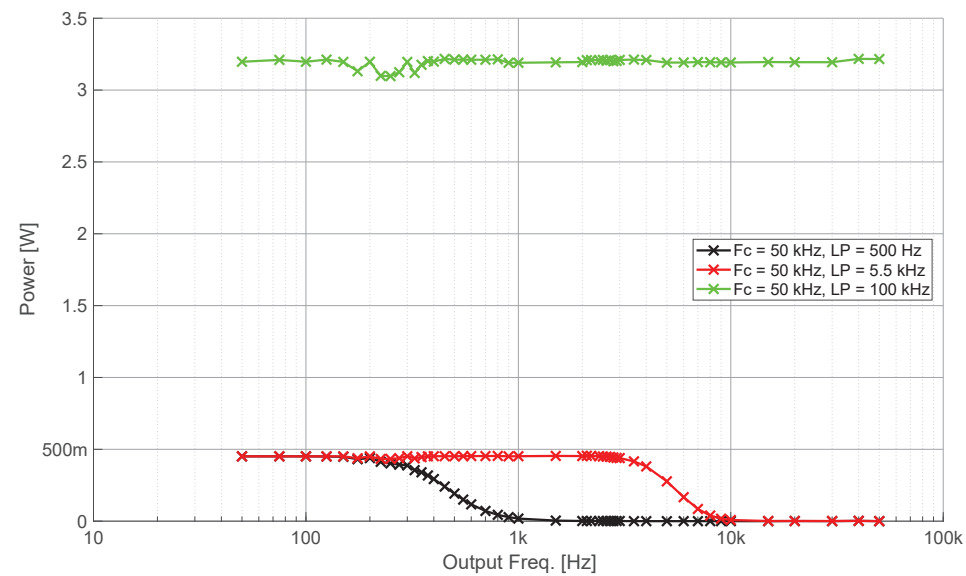

Figure 3.12: Measured output powers, for different filter configurations at a large set of different output frequencies. The carrier frequency is kept constant at $50 \mathrm{kHz}$

with a cutoff of $100 \mathrm{kHz}$. It shows the generator is capable of achieving high switching frequencies. Result for 11 different carrier frequencies are presented, varying from $50 \mathrm{kHz}$ to approximately $800 \mathrm{kHz}$. As can be seen, with increasing frequency the measured power is decreasing towards $0.5 \mathrm{~W}$. This is similar to the effect observed in Figure 3.12, when the carrier frequency is much higher than the cutoff frequency of the filter. Another interesting effect can be seen in different dead-time insertions. At higher switching frequencies, the dead-time inserted becomes more significant. Thus, reducing the amount of power at the output.

This subsection has shown the designed sPWM generator to be capable of generating high frequency $\mathrm{AC}$ signals at different switching frequencies. This has been verified by measuring output power with different filters. In the next section the generator is implemented in a multi-level converter demonstrator, which can (eventually) incorporate multi-frequency components as described in for instance [49].

\section{Multi Level converter}

Applying the sPWM generator to a setup similar as shown in Figure 1.4 will verify the intended use. However building the setup as depicted in Figure 1.4 will limit the evaluation to a single carrier frequency and only allows a DC/DC conversion as was shown in [10]. Therefore a simplified test setup was created that is shown in Figure 3.14. Compared to Figure 1.4, it can be seen that the filters and voltage source are removed, while the charged capacitors are represented by 2 ideal voltage sources $(10 \mathrm{~V})$. The measured voltage is applied to a load $R$ of $88 \Omega$. 


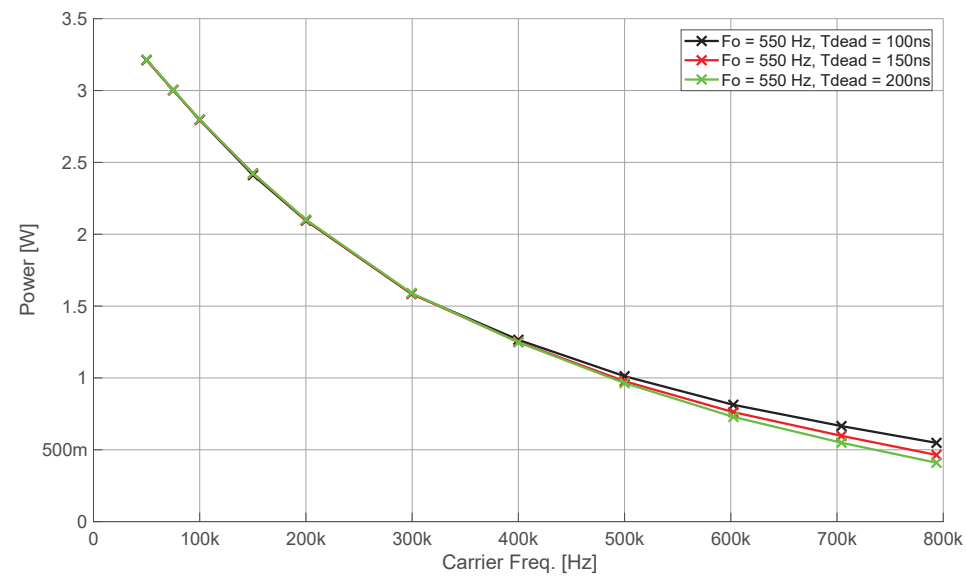

Figure 3.13: Measured output powers at different carrier frequencies with an LP filter cutoff at $100 \mathrm{kHz}$. The different lines represent different dead-times inserted: black $100 \mathrm{~ns}$, red $150 \mathrm{~ns}$, green $200 \mathrm{~ns}$.

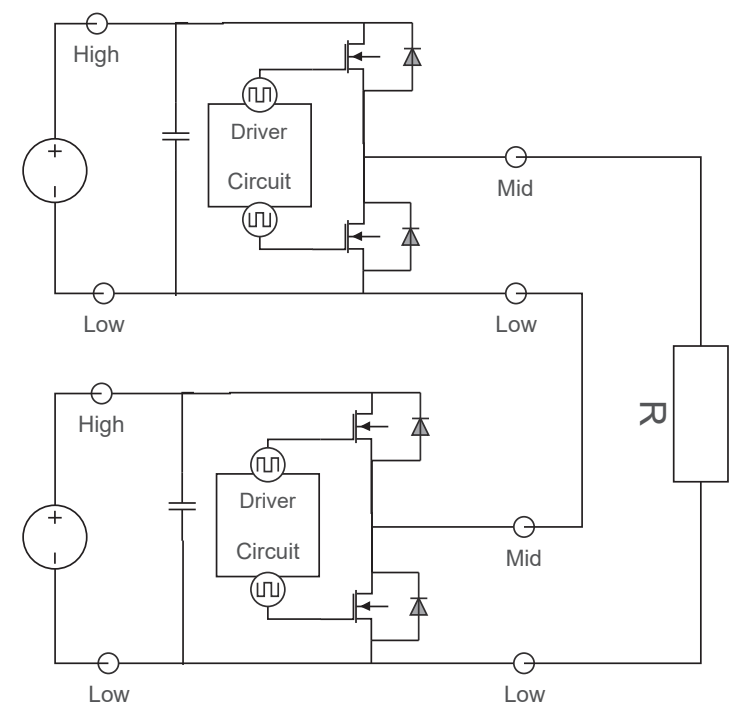

Figure 3.14: Simplified test setup for M3C. 
Figure $3.15 \mathrm{c}$ shows the measured output voltage, which consists of three levels as expected. The used settings were: $f_{c}=102 \mathrm{kHz}, f_{m}=23.7 \mathrm{kHz}$, $m_{i}=0.5, D_{i}=0.5$ and $t_{\text {dead }}=450 \mathrm{~ns}$

Figure 3.15 shows three waveforms that were collected asynchronously. This means the phases of each waveform (and each of their frequency components) are out of sync. They are not exactly the same, however they show similar trends. Note that the waveforms of Figure 3.15a and Figure 3.15b are created by adding the control signals of the upper switches inside the SM. As one can see, in all cases the pulses are not (perfectly) symmetrical nor identical, which is as expected. When $f_{c}$ is not an integer multitude of $f_{m}$, then the sinusoidal waveform from Figure 3.2 is being sampled differently every period of $f_{m}$. Together with a large, but common dead-time the signal will become non-symmetrical.

This section continues with measurements at higher switching frequencies, to push the setup to its limits. The setup is similar as in the previous measurement (Figure 3.14), however with slightly different settings: $f_{c}=2 \mathrm{MHz}$, $m_{i}=0.25, D_{i}=0.5$ and $t_{d e a d}=10 \mathrm{~ns}$. AC-amplitude was decreased to prevent the possible over-modulation, and the dead-time was decreased significantly. This can only be done for a short measurement at low-voltages. The $\mathrm{SiC}$ semiconductors used are rated for $1.2 \mathrm{kV}$ and a pulsed drain current of $80 \mathrm{~A}$, thus more than capable of surviving the short circuit of the $1 \mathrm{~A}$ limited power supply. Figure 3.16 shows 4 different high frequency output waveforms, that are highly distorted due to the high switching frequency. However one can clearly distinguish the three voltage levels and 'sinusoidal' output, thus verifying the operation of the high frequency and multilevel sPWM generator. 


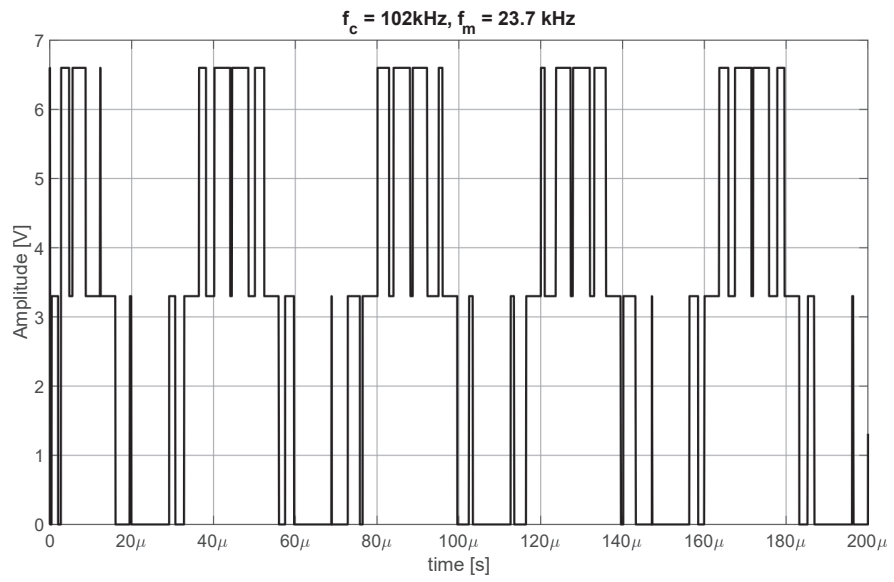

(a) Simulink result

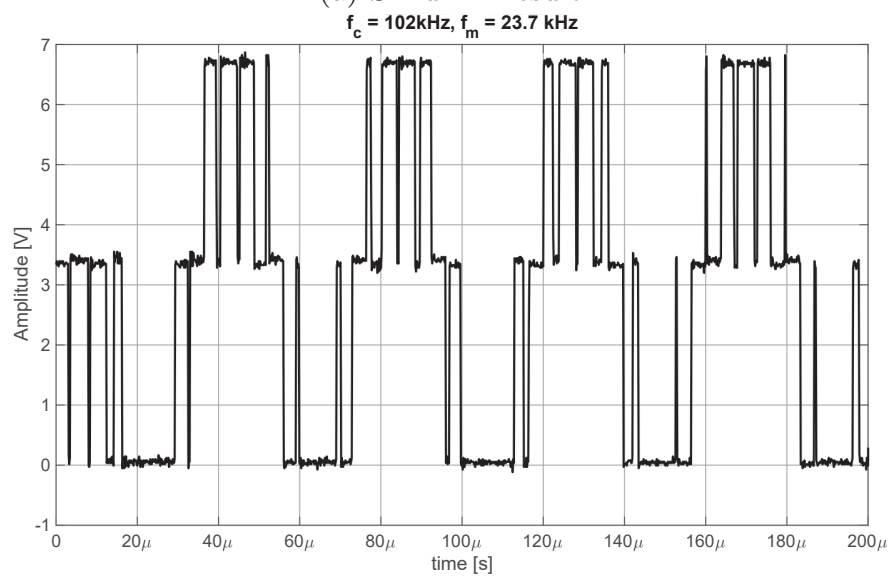

(b) Measured at Nexys3 output

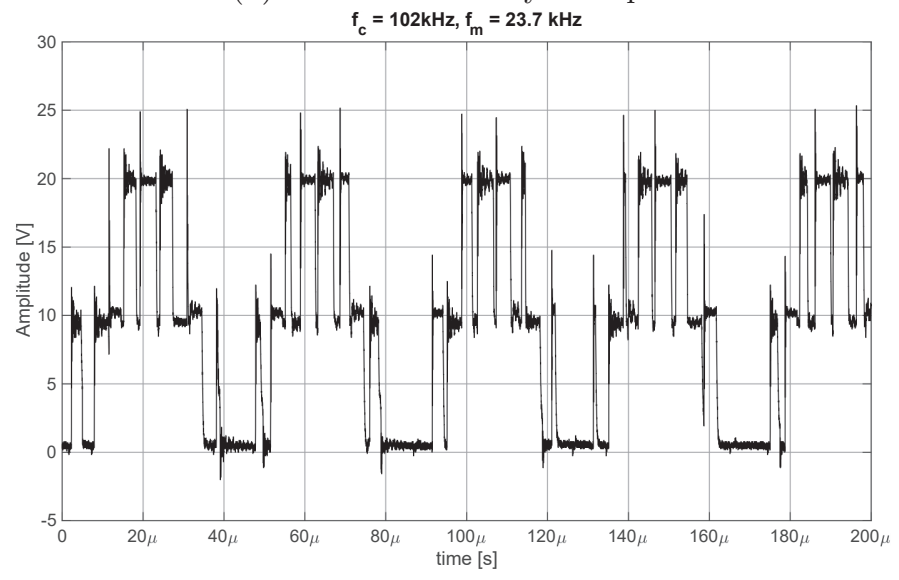

(c) Measured at the load (Figure 3.14)

Figure 3.15: First $200 \mu \mathrm{s}$ of the recorded waveform during time domain measurements of M3C simplified setup. 


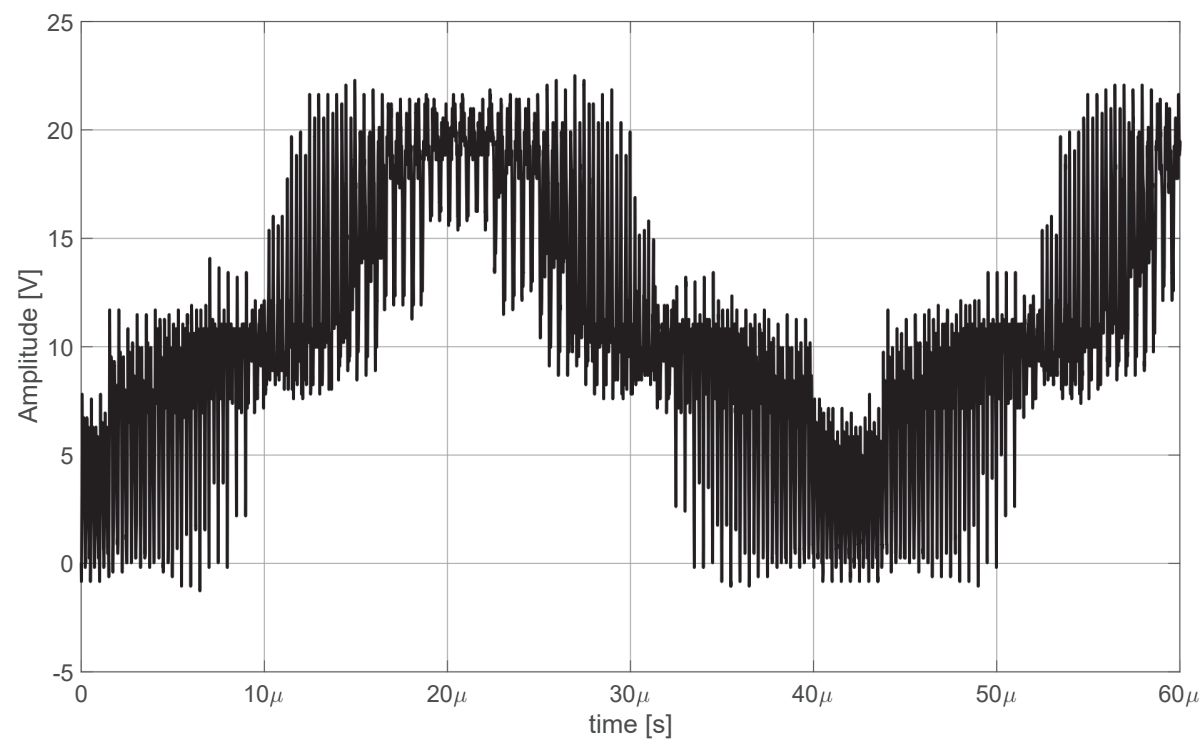

(a) $23.7 \mathrm{kHz}$

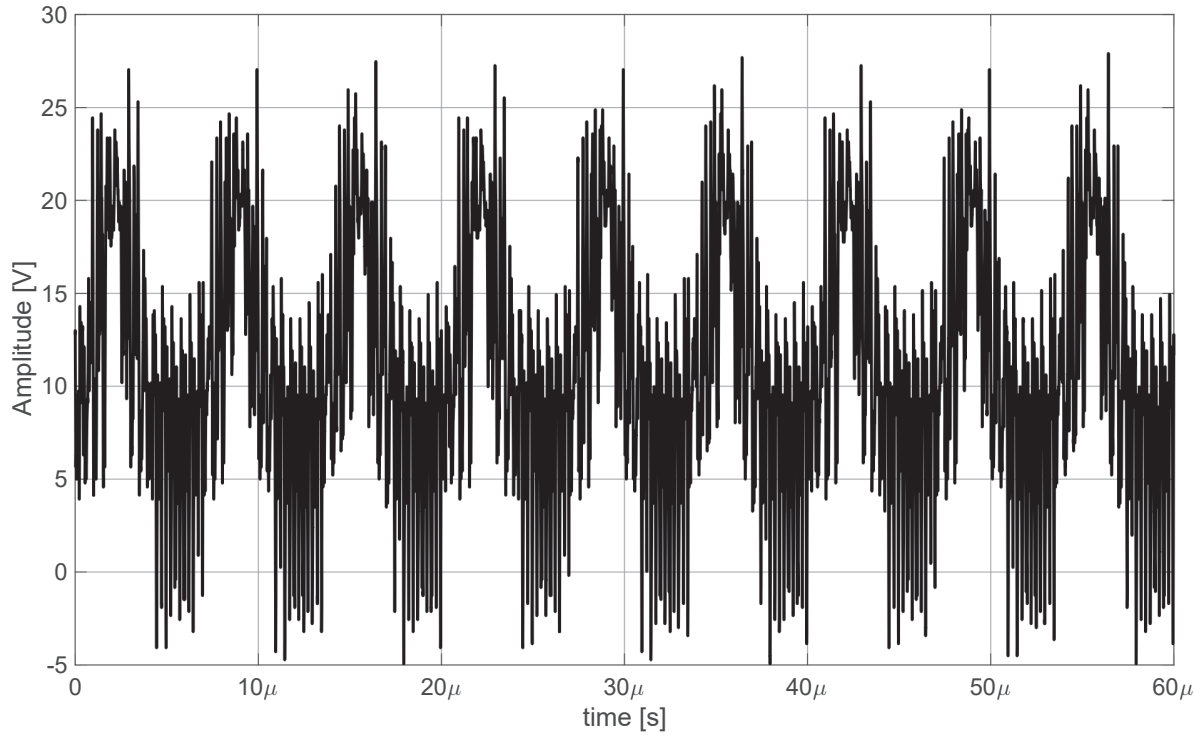

(b) $150 \mathrm{kHz}$

Figure 3.16: First $60 \mu$ s measured waveforms of simplified M3C setup using a $2 \mathrm{MHz}$ switching frequency creating different output frequencies. 


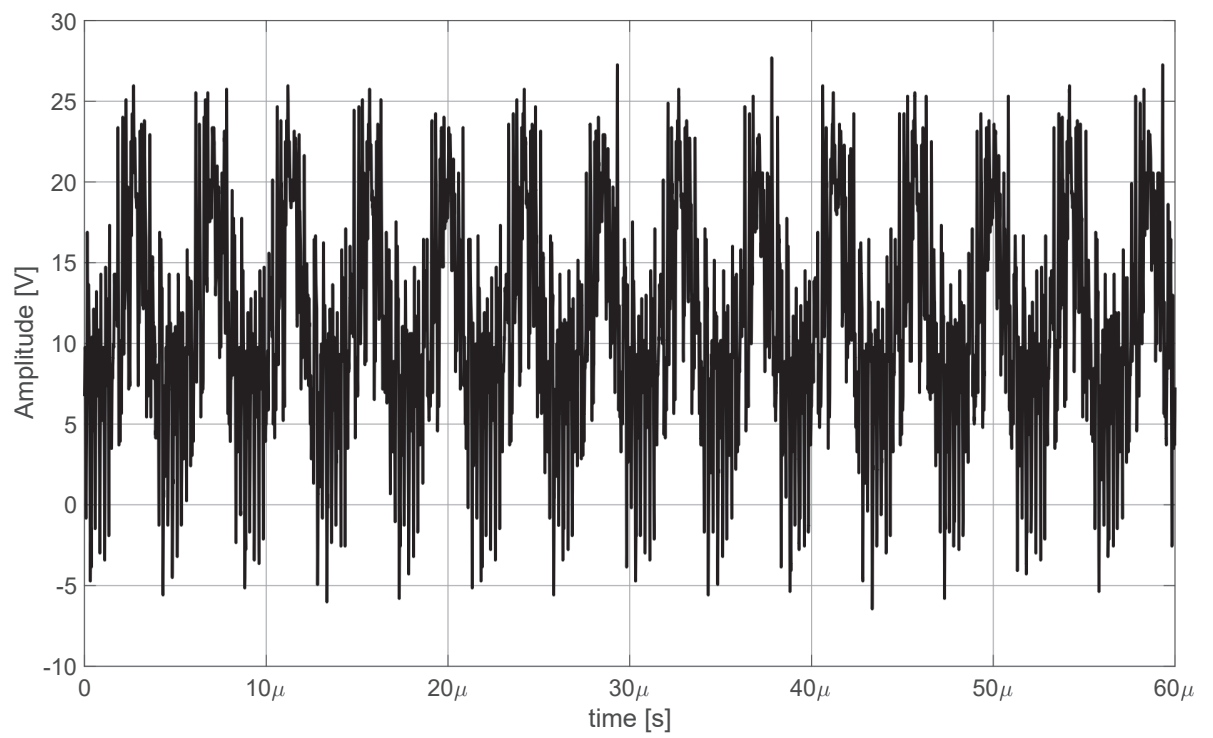

(c) $233 \mathrm{kHz}$

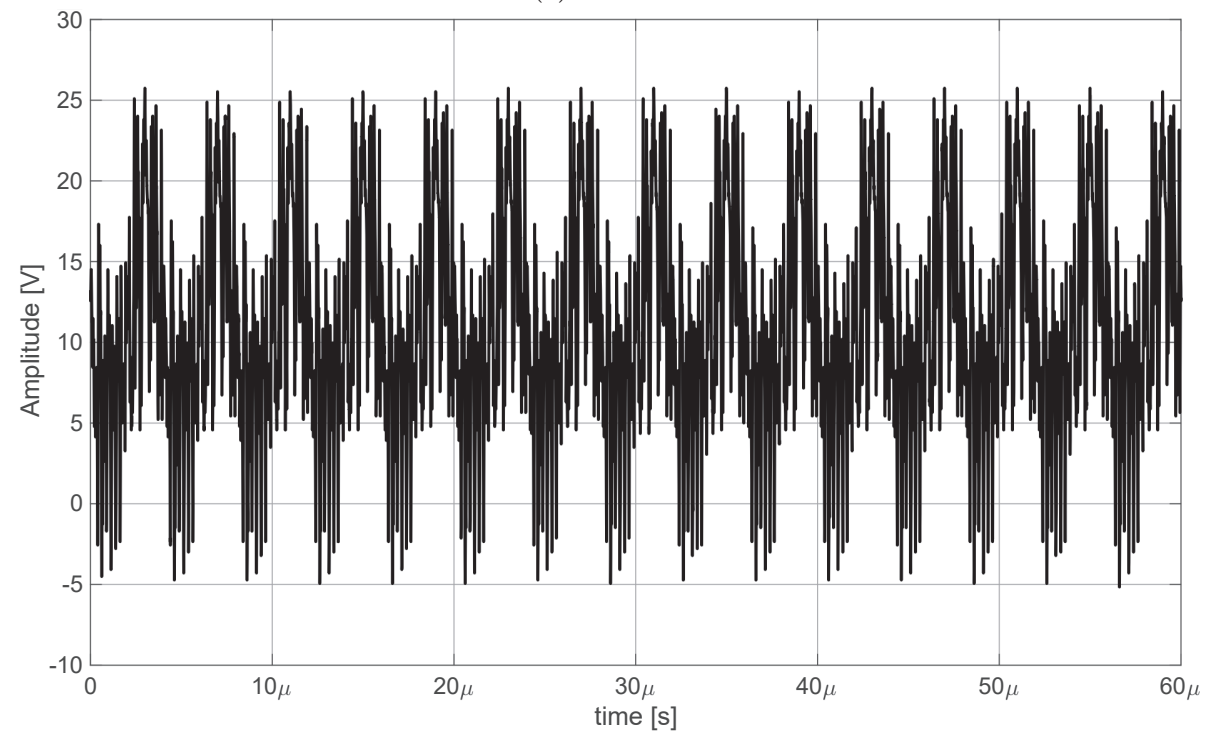

(d) $250 \mathrm{kHz}$

Figure 3.16: First $60 \mu$ s measured waveforms of simplified M3C setup using a $2 \mathrm{MHz}$ switching frequency creating different output frequencies. 
The sPWM generator has been shown capable of providing a research platform in a very wide sense. The signals have been verified on logical voltage levels as well as in simplified test setups. The development of the flexible generator has been done in parallel with the functional demonstrator that was created in Delft. The approach taken in Delft was focused on functional behavior and thus related to the transfer functions of the two operational modes attributed to the DC-M2C setup [10]. Relative to the flexible generator, the following parameters are fixed: Modulation frequency, switching frequency, and number of levels (i.e. SM). In the following section a study has been performed related to the conducted emission present in the $\mathrm{M} 3 \mathrm{C}$ demonstrator. The contribution of this section from an EMI point of view, is the effect of stacking SMs in series on the generated conducted emissions.

\subsection{Harmonic Cancellation}

In this section, a first characterization of conducted EMI within the novel M3C topology is investigated via measurements. Section 3.2.1 describes the prototype used to investigate the secondary power loops. Section 3.2.2 explains the different control parameters available within the demonstrator, which are directly related to the ones available in the developed signal generator. Compared to the previous chapter, the effects of the control parameters related to the transfer function are investigated. This is done by numerical simulations in section 3.2.3, as the theoretical model described in Chapter 2 was simplified to exclude the influence of phase-offset, DC-offset and AC-amplitude. Results from the investigation are verified in section 3.2.6 and 3.2.7. Firstly, two types of measurements are described, based on a single possible configuration of the prototype. Finally it will be shown that the harmonics of the secondary-loop frequency are of concern in the output as well as the higher switching frequency of the MOSFETS.

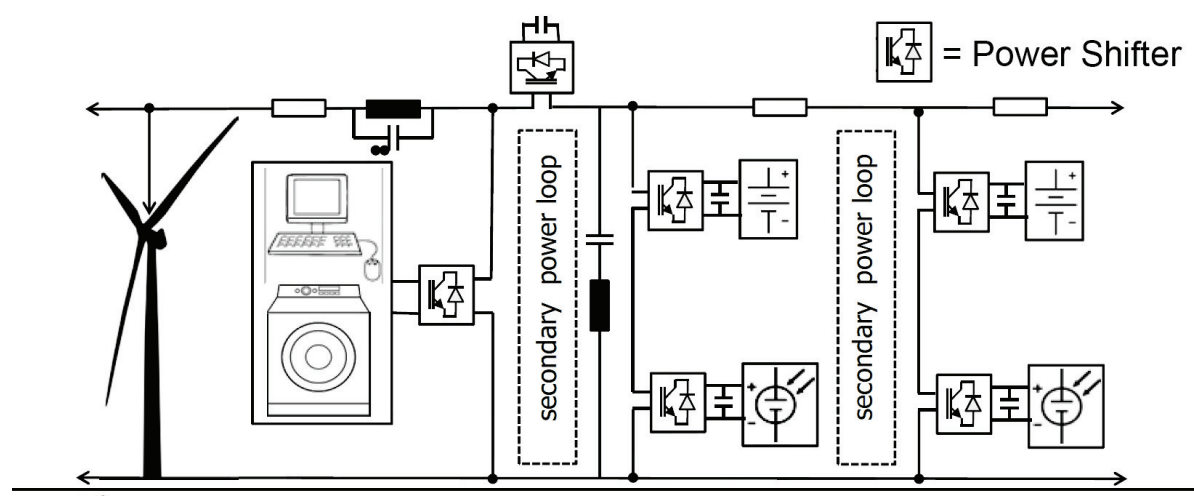

Figure 3.17: Envisioned High Voltage Direct Current (HVDC) Power Electronics System. 


\subsubsection{Demonstrator M3C}

A circuit model of the current prototype of the M3C, as developed by Delft University of Technology, can be seen in Figure 1.4. The device in question serves as a DC/DC converter with a secondary power loop tuned to $1.953 \mathrm{kHz}$. A simplified way of understanding how the converter operates is based on the assumption that a "sinewave" of $1.953 \mathrm{kHz}$ with a DC offset is generated inside the secondary loop. The sinusoidal part of the signal only exists within the secondary power loop. This is equivalent to the main $\mathrm{AC}$ component in conventional $\mathrm{M} 2 \mathrm{C}$ converters and is essential in order to balance the electrical charge across the sub-module capacitors. The "DC-offset" of the secondary power loop is provided by series connections of the four sub-modules, which, when configured properly, also provide the specified input and-or output voltage. They are drained by the load via the output filter.

\section{Fundamental Frequencies}

In this simplified view of the converter, one assumes only two existing frequencies. $f_{0}=D C$ and $f_{1}=1.953 \mathrm{kHz}$. However, to create the secondary loop, the circuit implements four cascaded sub-modules consisting of half-bridges together with three filters. A band-stop filter, a band-pass filter and an output filter. The half-bridges are driven by a PWM signal with a carrier frequency of $f_{s w}$. This carrier frequency is at least 10 times higher than $f_{1}$ due to the bandwidth and to limit the ripple on the capacitors. In this design, the switching frequency of the power MOSFETS (IRFB4127PbF) is $f_{s w}=24.4 \mathrm{kHz}$.

\section{Harmonics}

Next to these fundamental frequencies, higher order harmonics exist due to the switching nature of the device. The harmonics will be denoted as $H_{1, i}$ and $H_{s w, i}$, following the notation of the fundamental frequency of the secondary loop and switching frequency respectively. With $i$ denoting the order of the harmonic.

The existence of the two distinct fundamental frequencies and their harmonics is characteristic of sPWM. Like in conventional systems, leakage of additional harmonics are generally undesirable due to decreased efficiency, control issues and EMI [3]. Leakage reduction by implementing filters is common practice.

\section{Filters}

As the new topology provides unique challenges for filtering, we will distinguish between power frequency filters and EMI-filters. The challenge in filtering arises from the fact that many (sub-)modules can share the same filter, and series modules can experience large dv/dt's. Another unique challenge is the voltage rating a single filter has to have. Due to stacking of submodules, the filters can be subject to voltages in the range of $\mathrm{kV}$ 's, depending on the application. Note 
that in the prototype only power frequency filters have been implemented, with no regard for the EMI performance of the converter.

\subsubsection{Power Shifter Module}

A power shifter module may consist of multiple Sub-Modules (SM's) stacked to provide the desired voltage levels. In Figure 3.17 the power shifter is depicted by a single submodule. However, for the HVDC systems, this will in practice represent a stack of multiple SM's, due to the limitation of the voltage on the capacitors and/or the current through a single IGBT/MOSFET. This can be solved by cascading and cascoding SMs respectively. The prototype created as a proof of concept for the M3C consists of four cascaded SM's. The prototype is create by K. Huang and studied in [10], where the focus is on the two possible operational modes of the M3C. These modes are considered to be one of the control parameters that show the modularity of the topology. Apart from the chosen operational mode, several parameters have been identified and described in the following subsection for the purpose on control.

\section{Power flow direction}

The direction of power flow can be switched between the following two modes. Either the power flows from high voltage side to the lower voltage side or from the lower side to the higher side, referred to as High-to-Low (HTL) and Lowto-High (LTH) respectively.

\section{Coupling Mode}

Based on [10] the multilevel converter has two operational modes. Resistive Coupled Mode (RCM) and Inductive Coupled Mode (ICM). In RCM the converter acts as a Voltage Controlled Voltage Source (VCVS), while in ICM it operates as a Voltage Controlled Current Source (VCCS).

\section{Input/output Voltage ratio}

The multilevel converter has 4 control parameters in RCM and 6 control parameters in ICM. These parameters control voltage transfer ration and/or the output current, depending on the mode of operation. The details of which are shown in [10] and will be neglected in this study, due to the implementation of the prototype that has been previously presented. A predefined set of control parameters is programmed into the FPGA that controls the sub-modules. At a macro-scale, the relevant aspects for this analysis are the voltage ratios, not the exact values of the control parameters required to achieve this ratio.

\section{Modularity: Order of load/source complexity}

At this point, the complexity of the M3C system appears in the foreground. The system is build in such a way that it is possible not only to transfer power 
from HTL or LTH, but also between sources and loads connected directly to a submodule. This means that controlling the capacitor voltage balance actually enables sources and loads to be connected per module. This increases the possible EMI-nightmare since the number of submodules implemented in series and/or parallel is not limited.

\section{Spectrum of Single Power Shifter Module}

In this subsection, a spectral analysis will be preformed to eventually assess the dependency of the power spectrum on the voltage transfer ratio. Every submodule consists of a half-bridge with two power MOSFETs, as can be seen in Figure 1.4. Each of the sub-modules can be considered an individual noise source, which was investigated in chapter 2. The spectral characteristics of these noise sources are determined in section 3.2 .3 by numerical methods. In contrast to the mathematical model derived in Chapter 2, the numerical model includes a study into the effect of the parameters of the modulating waveform, i.e. the amplitude, phase and DC-offset compared to the reference triangular wave. As was presented in [50] obtaining an analytical expression for the harmonics (and subharmonics) is quite cumbersome.

In case of the studied demonstrator, the control signal is generated by a comparator receiving a triangular wave of $24.4 \mathrm{kHz}$ and a sinusoidal wave of $1.953 \mathrm{kHz}$. In this analysis, we assume the triangular wave to be a fully known ideal signal. This signal does not change over the different modes of operation, available in the prototype of the M3C. The effects of a non-ideal reference signal on the EMI spectrum is shown in [51].

The sinusoidal signals used to generate the PWM signal contains the control parameters that eventually determine the voltage transfer ratio. Equations for the reference signals, as defined in [10] are restated in this section, together with the transfer ratios. This demonstrates that, by controlling either the ACand/or DC-component of the reference signals, the voltage transfer ratio is adjusted. Note however that Equation 3.6 only holds for resistive coupled mode. While Equation 3.7 is a simplification and holds for the inductive coupled mode.

$$
\begin{gathered}
u_{r e f, u}=D_{u}-m_{u} \cdot \cos (\omega \cdot t+\phi) \\
u_{r e f, l}=D_{l}+m_{l} \cdot \cos (\omega \cdot t) \\
\frac{V_{o}}{V_{i}}=1+\frac{D_{u} \cdot m_{l}}{D_{l} \cdot m_{u}} \\
\frac{I_{o}}{V_{i}}=\frac{m_{u} \cdot m_{l} \cdot \sin (\phi)}{2 \omega \cdot D_{l} \cdot D_{u} \cdot\left(L_{a r m_{u}}+L_{a r m_{l}}\right)}
\end{gathered}
$$

The parameters used in Equations 3.4 to 3.7 are listed in Table 3.3. Equations 3.4 and 3.5 depict the reference voltage for the upper and lower SMs respectively, while Equation 3.6 gives the voltage transfer ratio and Equation 3.7 is the ratio of output current with respect to input voltage. In the case of the prototype $\omega=2 \cdot \pi \cdot f_{s e c}, D_{u}$ and $D_{l}$ are the DC components of the reference 
Table 3.3: Parameters of the M3C circuit model as adopted from [10].

\begin{tabular}{|c|c|}
\hline$V_{i}$ & Input DC voltage \\
\hline$V_{o}$ & Output DC voltage \\
\hline$I_{o}$ & Output DC current \\
\hline$u_{r e f, u}$ & Upper Reference signal \\
\hline$u_{r e f, l}$ & Lower Reference signal \\
\hline$D_{u}$ & Upper DC offset \\
\hline$D_{l}$ & Lower DC offset \\
\hline$m_{u}$ & Upper modulation index \\
\hline$m_{l}$ & Lower modulation index \\
\hline$L_{a r m_{u}}$ & Upper arm inductor \\
\hline$L_{a r m_{l}}$ & Lower arm inductor \\
\hline
\end{tabular}

signals, $m_{u}$ and $m_{l}$ the AC components (amplitudes) of the signal and $\phi$ is the phase offset between the two reference signals. In the following section, an analysis of the spectrum's dependency on amplitude, dc-offset and phase is given. Note that for resistive coupled mode, the phase offset between upper and lower reference signals is $\phi=0$. Also in case of Equation 3.7 the input voltage is corrected for the voltage drop across the input resistance due to the input current.

\subsubsection{Spectrum of PWM}

The analysis of the spectrum is performed using terminology adopted from the field of communications engineering as used for instance in [32]. In this setup, the PWM signal is the result of a comparator with two input waves, a triangular and sinusoidal waveform, which have a frequency of $f_{c}=24.4 \mathrm{kHz}$ and $f_{m}=1.953 \mathrm{kHz}$ respectively. The generated sinusoidal wave is:

$$
x(t)=A \cdot \sin \left(2 \cdot \pi \cdot f_{m} \cdot t+\phi\right)+D C
$$

In the following analysis the carrier and modulation signals are presented. However, the spectrum is derived from the "rectangular" PWM, resulting from the comparator's output. The most pronounced harmonics and subharmonics were empirically determined from the parameter sweeps shown in Figures 3.4, 3.19 and 3.20. Based on this, we defined the following frequencies of interest.

Fundamental Frequencies: $f_{m}, f_{c}$

Harmonics: $f_{m} \cdot i, f_{c} \cdot i$

Sub-Harmonics: $f_{c} \cdot i \pm f_{m} \cdot j$ 


\section{Amplitude}

In the case of the amplitude analysis the parameters in Equation 3.8 were set to $D C=0.5, \phi=0$ and $A=[0.01,0.11,0.21,0.31,0.41,0.51]$. The amplitude was changed according to the values shown in the vector. Figure 3.18a shows the increasing modulation amplitude with respect to the carrier signal, consisting of triangular waveform.

Figure $3.18 \mathrm{~b}$ shows, that increasing the amplitude of the modulation signal actually increases the power flowing into the odd numbered sub-harmonics. Notice the even numbered sub-harmonics are orders of magnitude weaker than their odd numbered counterparts. Another noticeable feature is the amount of power in the sub-harmonics around the even numbered harmonics of the carrier frequency. The first sub-harmonics appear to constantly have more power than the corresponding even harmonic. When increasing the amplitude of the sine wave, these sub-harmonics tend to become more dominant, while simultaneously reducing harmonics.
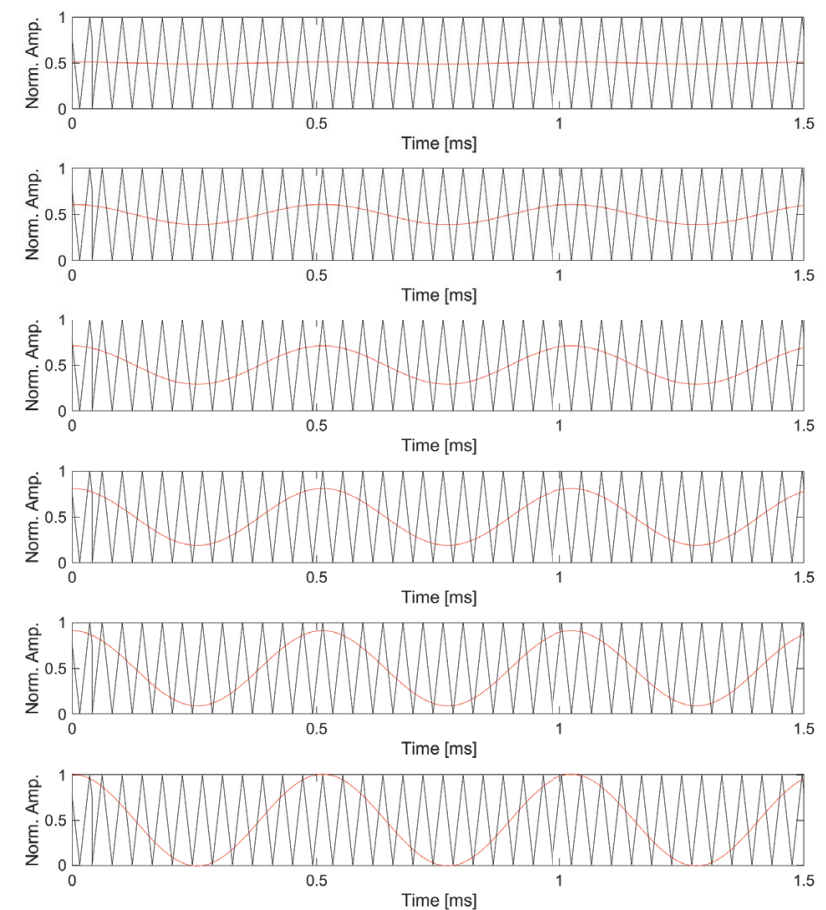

(a) Time signals

Figure 3.18: Amplitude dependency. 

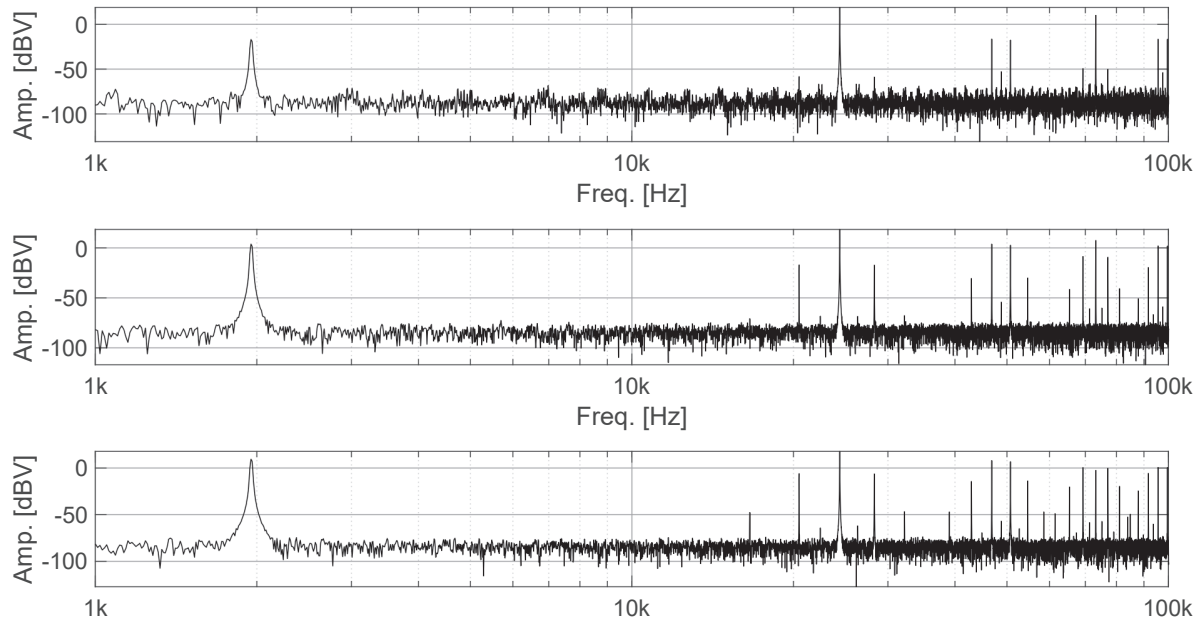

Freq. [Hz]
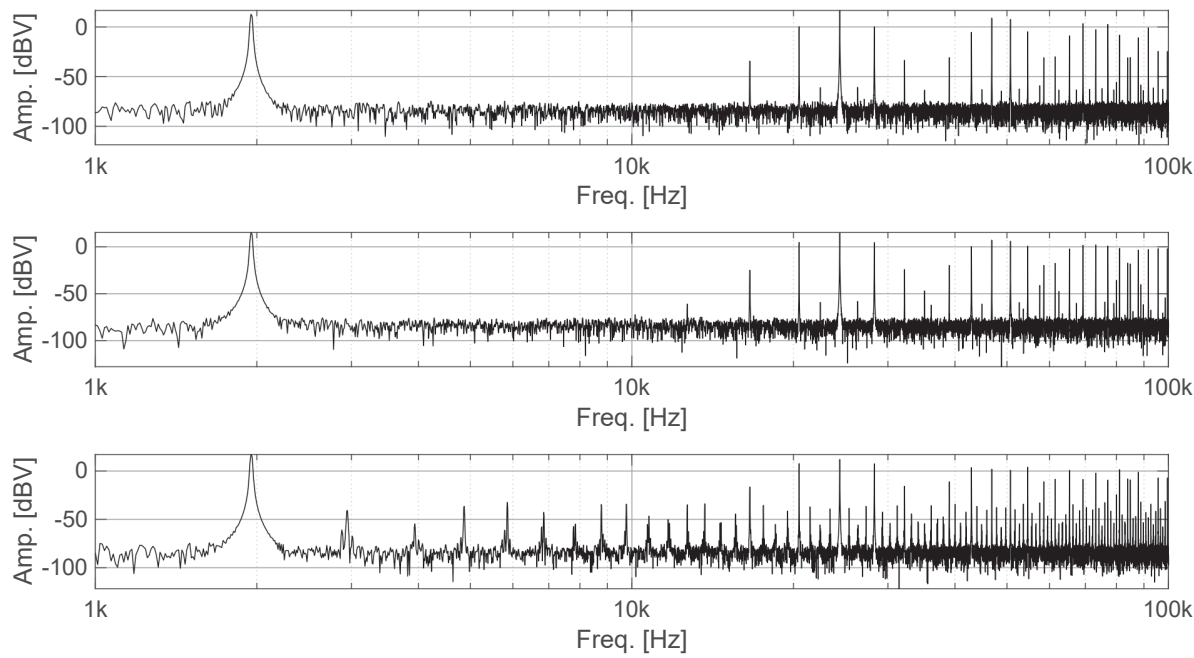

(b) Spectra

Figure 3.18: Amplitude dependency. 


\section{DC-offset}

In the case of the DC-offset analysis in Equation 3.8, A=0.25, $\phi=0$ and $D C=[0,0.2,0.4, .6,0.8,1]$. Figure $3.19 \mathrm{~b}$ shows in situation $1,2,5$ and 6 more dominant frequencies arise during clipping phenomena. This behavior was also visible in situation 6 of Figure 3.18b and is a result of over-modulation. A noticeable difference however is the fact that the sine wave in case of the DC-offset sweep is placed non-symmetrically around the mean of triangular wave. This offset remained symmetric during the amplitude sweep. From this, it can be concluded that the even numbered subharmonics become dominant due to this non-symmetrical placement of the sine wave. Also, the even numbered harmonics of the carriers seem to become more dominant in the non-symmetrical situation.
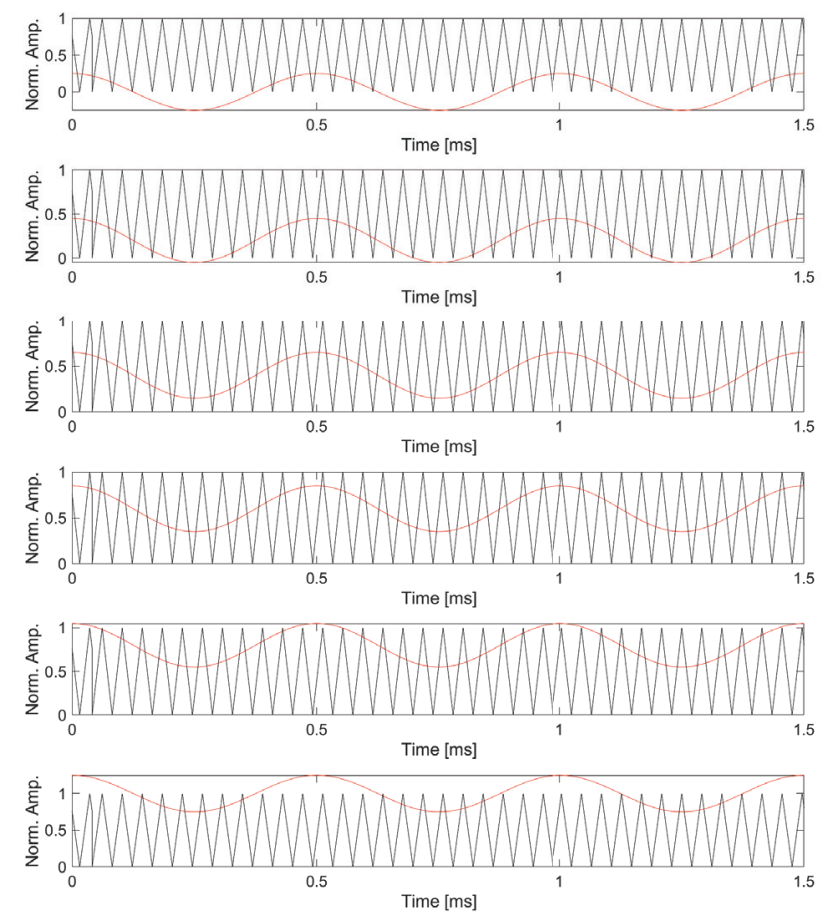

(a) Time signals

Figure 3.19: DC offset dependency. 

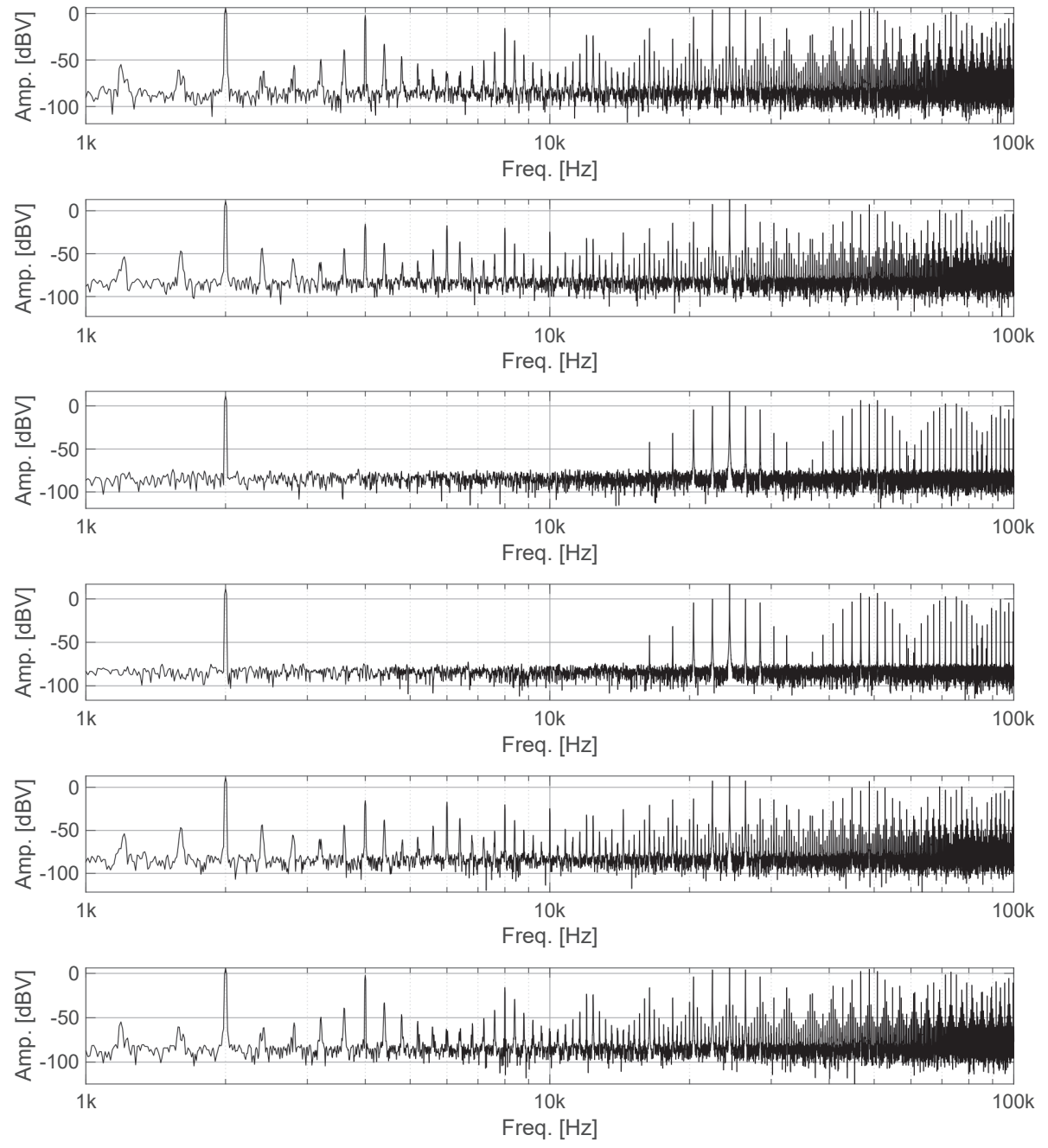

(b) Spectra

Figure 3.19: DC offset dependency. 


\section{Phase}

For completion of the analysis, a phase sweep was also performed. Even though it is expected to have no impact on the power spectrum of the PWM signal. It was done to show that the spectrum will not alter, despite the period of the carrier not being a multiple of the sinusoidal wave period. The phase sweep would introduce a linearly increasing or decreasing phase shift between the carrier and modulation signal. In the case of the phase analysis in Equation 3.8, $A=0.25, D C=0.5$ and $\phi=\left[0, \frac{2 \pi}{5}, \frac{4 \pi}{5}, \frac{6 \pi}{5}, \frac{8 \pi}{5}, 2 \pi\right]$. Figure $3.20 \mathrm{~b}$ shows comparable spectral peaks in all six situations, which is similar to situation 3 of the amplitude sweep. Note that here the phase between the carrier and modulation signal is investigated. Likewise, the phase difference between reference signals of different submodules. This becomes interesting when the cascading of multiple SM's is investigated.
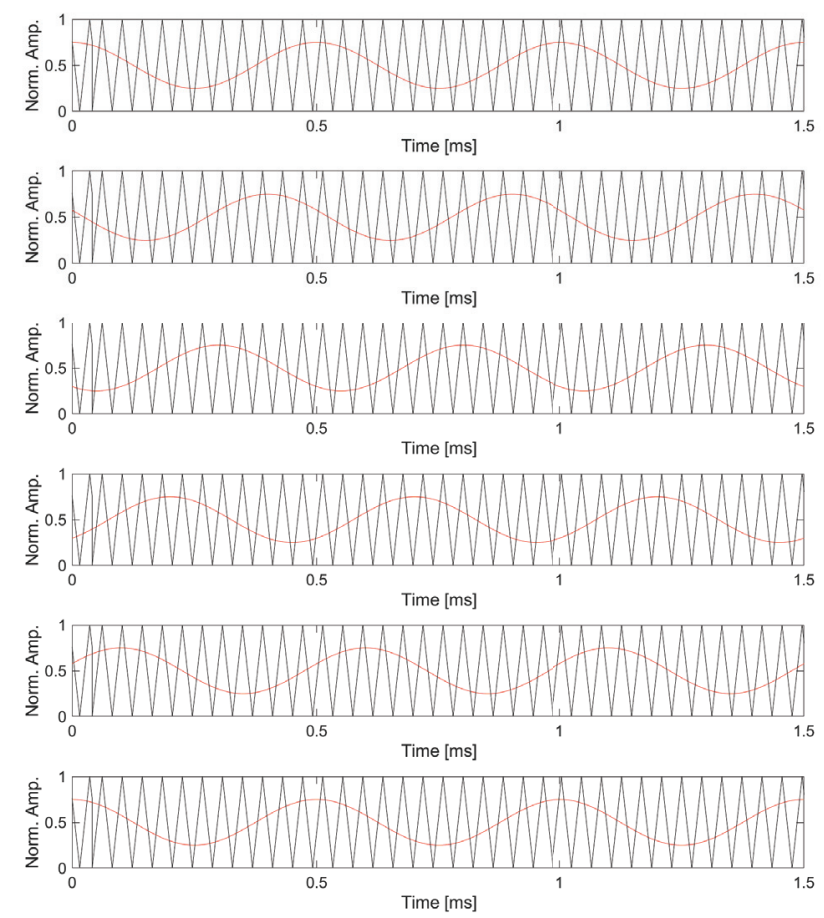

(a) Time signals

Figure 3.20: Phase dependency. 

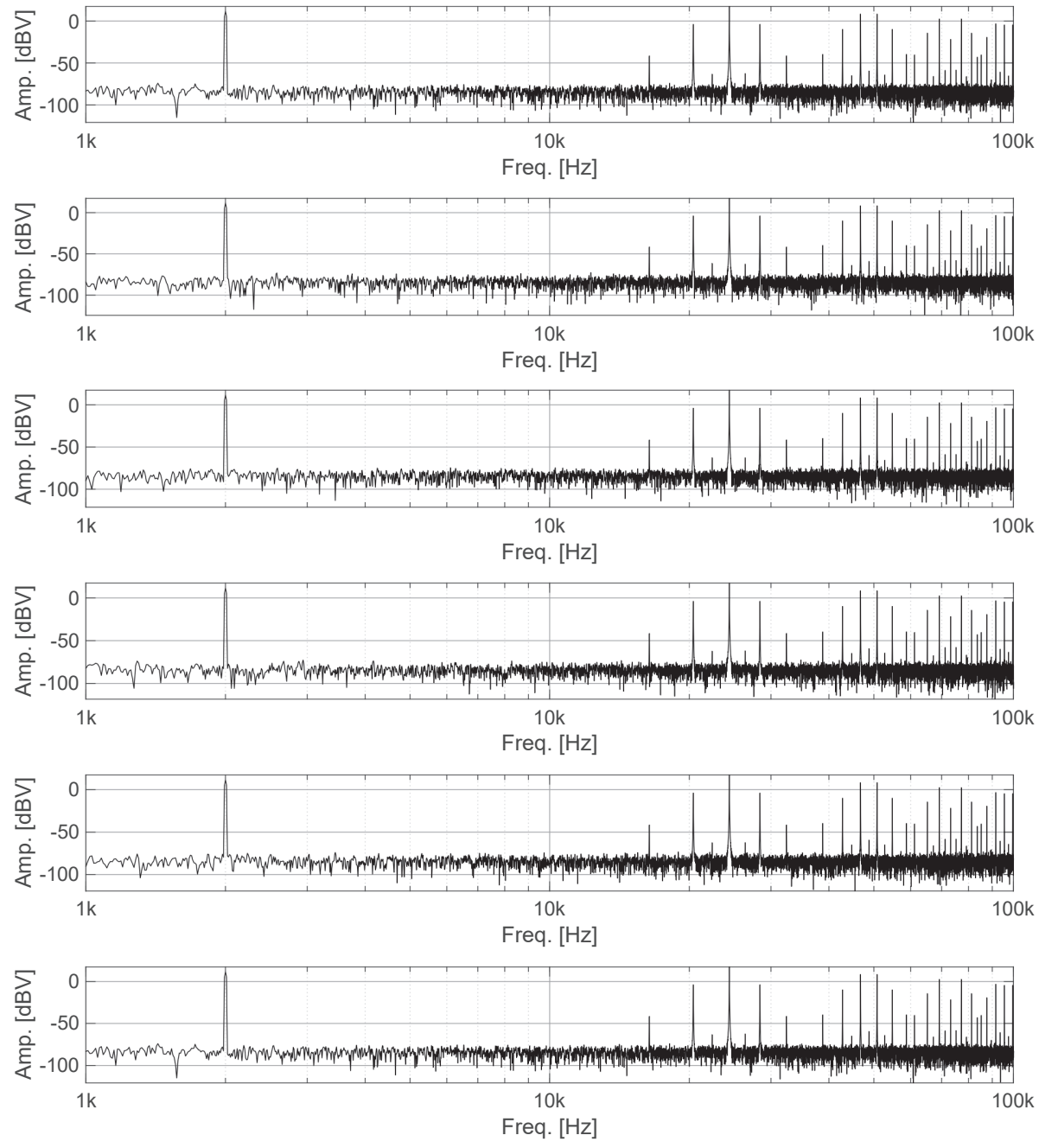

(b) Spectra

Figure 3.20: Phase dependency. 


\subsubsection{Numerical Analysis Summary}

To summarize the findings in this numerical analysis, we can identify the following phenomena:

1. Symmetric: when the sinusoidal waveform is symmetrically placed within the triangular waveform, the even numbered subharmonics are suppressed.

2. Asymmetric: when the sinusoidal waveform has a dc-offset compared to the mean of the triangular waveform, the odd numbered subharmonics are in the spectrum.

3. Increasing the amplitude of the sinusoid, the subharmonics will become more significant.

A qualitative analysis of the noise produced by a single submodule has been given, based on the variable parameters of the driving signals. The parameter sweeps were confined to the cases that are available in the M3C prototype. In the following section, the numerical analysis of the EMI spectrum generated in a multi-submodule system is continued.

\subsubsection{Harmonic Cancellation in Multi SM converter}

It was demonstrated in the analysis of a single SM that both fundamental frequencies and their (sub)-harmonics were all present in the spectra. In the following analysis, multiple SM's are cascaded, therefore their voltages are superimposed as shown in the equivalent circuit diagram in Figure 3.21. It is assumed here that every SM has an ideal voltage source attached (shown as capacitors), that is either bypassed or inserted into the secondary frequency loop as shown in Figure 1.4. In the numerical results, there is no dead-time inserted and all signals are assumed ideal. i.e. no ringing, no rise time, and no fall time. In Figure 3.22 the results are shown from $1 \mathrm{kHz}$ to $1 \mathrm{MHz}$. As

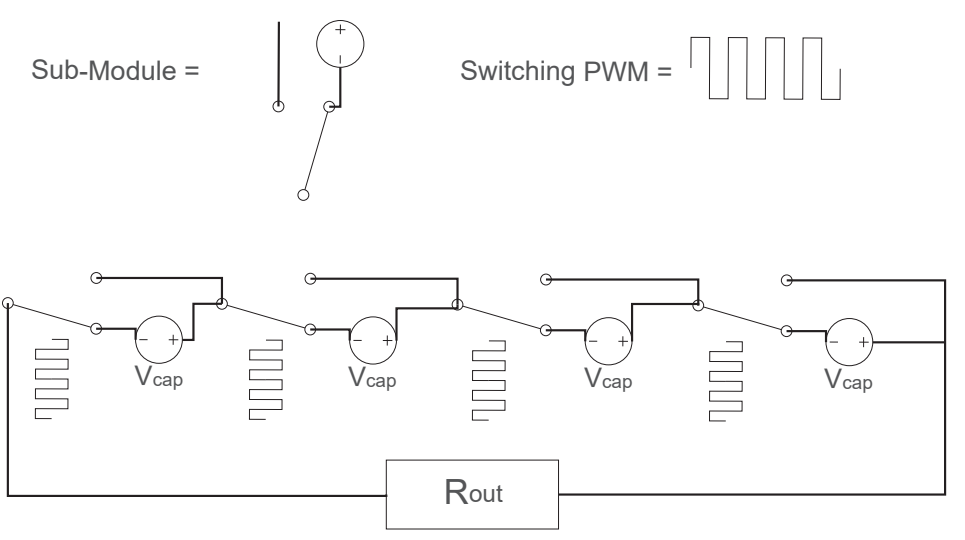

Figure 3.21: Equivalent circuit diagram. 
can be seen, a single SM will result in all frequencies as defined in section 3.2.3 to be present. When a second SM is inserted into an arm, a cancellation of the carrier frequency $\left(f_{c}=25 \mathrm{kHz}\right)$ and its even numbered harmonics and their sub-harmonics is seen. This Harmonic Cancellation effect can even be extended. With the insertion of a third SM, it is possible to cancel the carrier and the first harmonic, while the second harmonic is the 'first' frequency present. Note the effect is a result of the multiphase-carrier scheme. By phase shifting the triangular waveform, the reference sinusoidal waveform is sampled at different moments in time by each carrier. This can be seen in Figure 3.23.

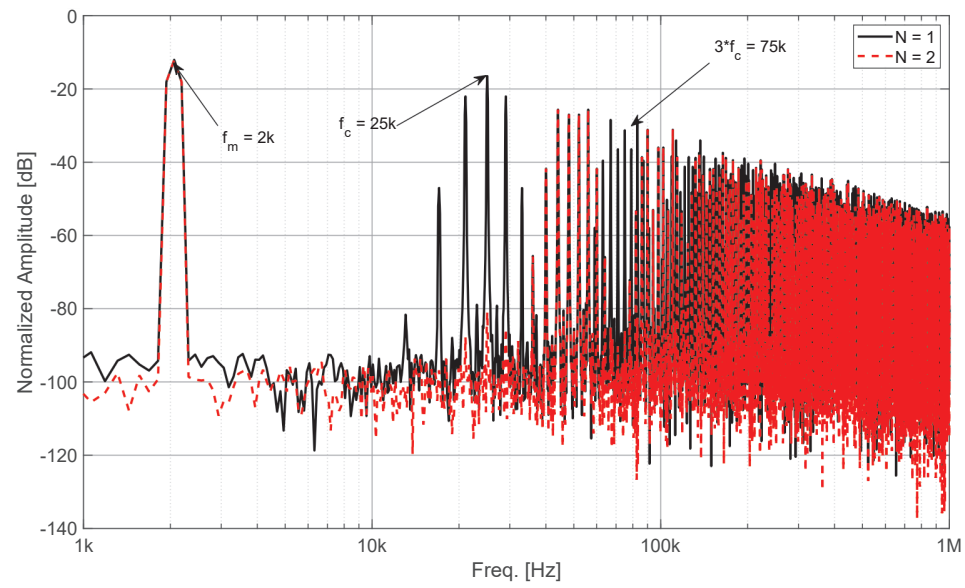

(a) One and two SMs cascaded.

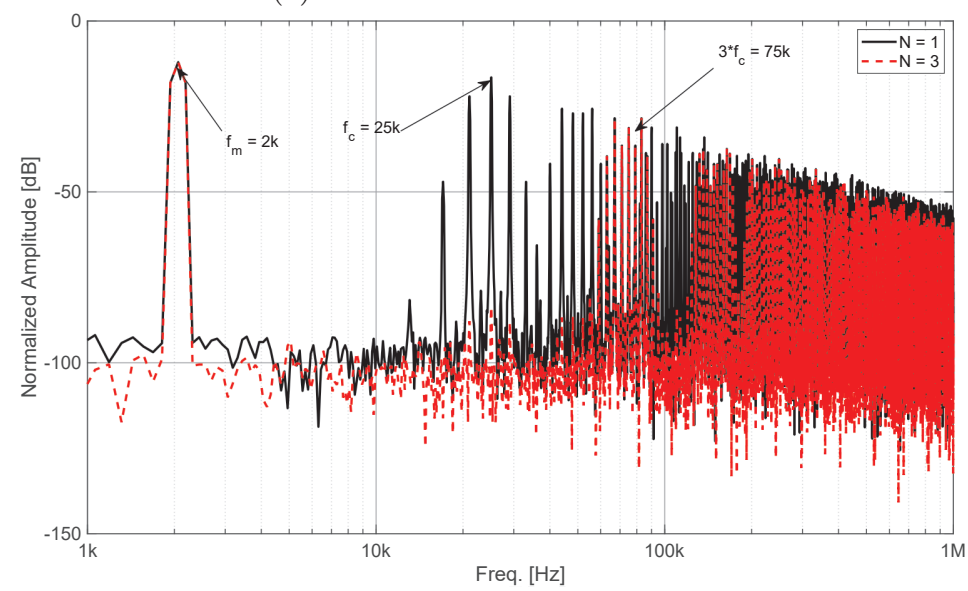

(b) One and three SMs cascaded.

Figure 3.22: Spectral densities of cascaded sub-modules with according phase offsets between the reference signals. 
Here the amplitude of the resulting arm voltage is normalized to the number of SMs used. In this time domain analysis, it can be easily seen that switching events are occurring more often with the increasing collection of SMs. Also more voltage levels become available by increasing the number of SMs. This results in a couple of implications. By increasing the number of SMs, the noise generated due to switching events will shift towards higher frequencies. From a power electronics designer point of view, this allows the SMs to be operated at lower switching frequencies, which decreases switching losses, while the passive components used can still remain small, as the total system is still running on a high switching frequency. To verify this numerical analysis, a measurement was implemented using the demonstrator. In the next section, the measurements performed on the converter are explained.

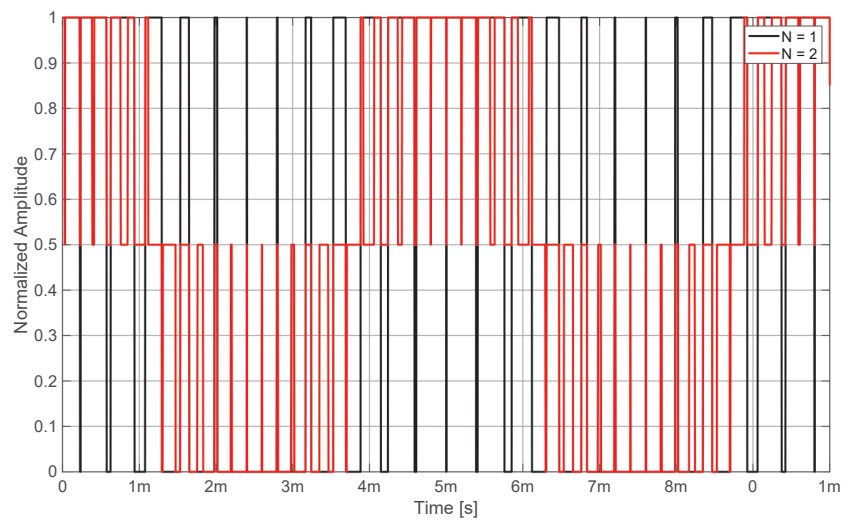

(a) One and two SMs cascaded.

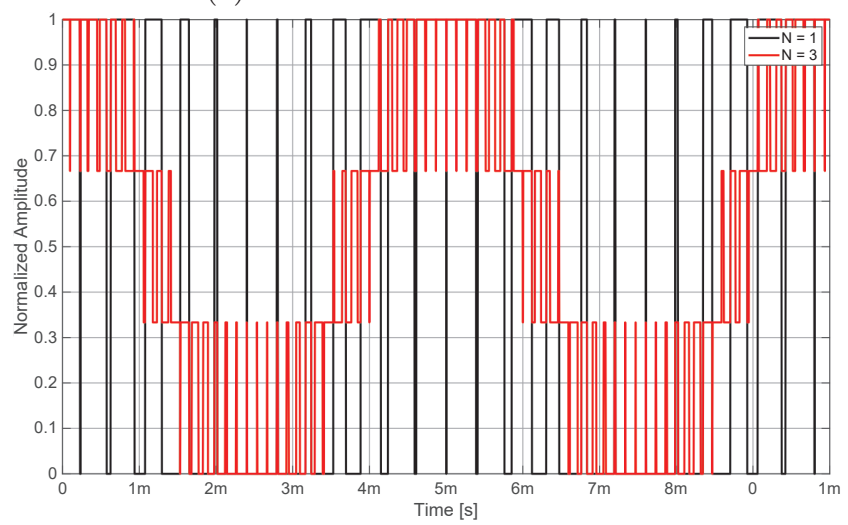

(b) One and three SMs cascaded.

Figure 3.23: Time domain simulation cascaded sub-modules. 


\subsubsection{M2C-DC/DC Demonstrator}

The complexity of the system increases exponentially by increasing the number of submodules, loads, sources and frequencies used in the system. Here the characterization was confined to possibilities provided by the prototype of the M3C. Note that the demonstrator was built without any regard for EMI, only some basic automated Printed Circuit Board (PCB) checking tool was used. The characterization of the EMI produced in this setup will be shown, according to different settings within the same configuration. For the sake of simplicity we have decided to start with only a single load and a single source. We maintain the power flow direction from low to high voltage side and use the converter as a VCVS. i.e. the mode of operation is RCM. Two independent measurements where performed:

1. Input/Output (I/O) measurement

2. Lower arm measurement

During the I/O measurements the transfer voltage ratio is varied according to Table 3.4, while in the lower arm, only modes 1 through 3 where measured. " $D "$ and " $m$ " are integer values due to the FPGA implementation. Using Equation 3.6, one can predict the transfer ratio. The resulting measurement configurations can be seen in Figure 3.24.

Table 3.4: Transfer Voltage Ratios.

\begin{tabular}{|l||c|c|c|c|c|c|c|}
\hline Mode & $V_{\text {in }}[\mathrm{V}]$ & $V_{\text {out }}[\mathrm{V}]$ & Ratio & $D_{u}$ & $D_{l}$ & $m_{u}$ & $m_{l}$ \\
\hline 1 & 29.41 & 39.58 & 1.35 & 1024 & 3072 & 6 & 7 \\
\hline 2 & 29.39 & 41.94 & 1.43 & 1024 & 3072 & 5 & 7 \\
\hline 3 & 29.36 & 45.89 & 1.56 & 1024 & 3072 & 4 & 7 \\
\hline 4 & 29.30 & 53.34 & 1.82 & 1280 & 2816 & 5 & 9 \\
\hline 5 & 29.22 & 61.95 & 2.12 & 1536 & 2560 & 6 & 11 \\
\hline 6 & 29.16 & 68.86 & 2.36 & 1792 & 2304 & 7 & 13 \\
\hline 7 & 29.01 & 78.07 & 2.69 & 1792 & 2304 & 6 & 13 \\
\hline 8 & 28.91 & 83.20 & 2.88 & 2048 & 2048 & 11 & 15 \\
\hline
\end{tabular}

The schematic representation of the two measurement setups can be seen in Figure 3.24, while Figure 3.25 shows the actual prototype with measurement equipment as used in the $\mathrm{I} / \mathrm{O}$ measurement. This equipment is depicted in Figure 3.25 and consists of the following:

- 4 channel Osciloscope: Yokogawa DLM2000

- Current Probes: Yokogawa 701929 (BW: DC to $50 \mathrm{MHz}$ )

The measurements are all preformed at $62.5 \mathrm{Msamp} / \mathrm{s}$ for $20 \mathrm{~ms}$, which provides 125000 samples. A periodogram is created, showing the frequency range from $1 \mathrm{kHz}$ to $30 \mathrm{MHz}$. The scopes where used in AC coupled mode, therefore the 


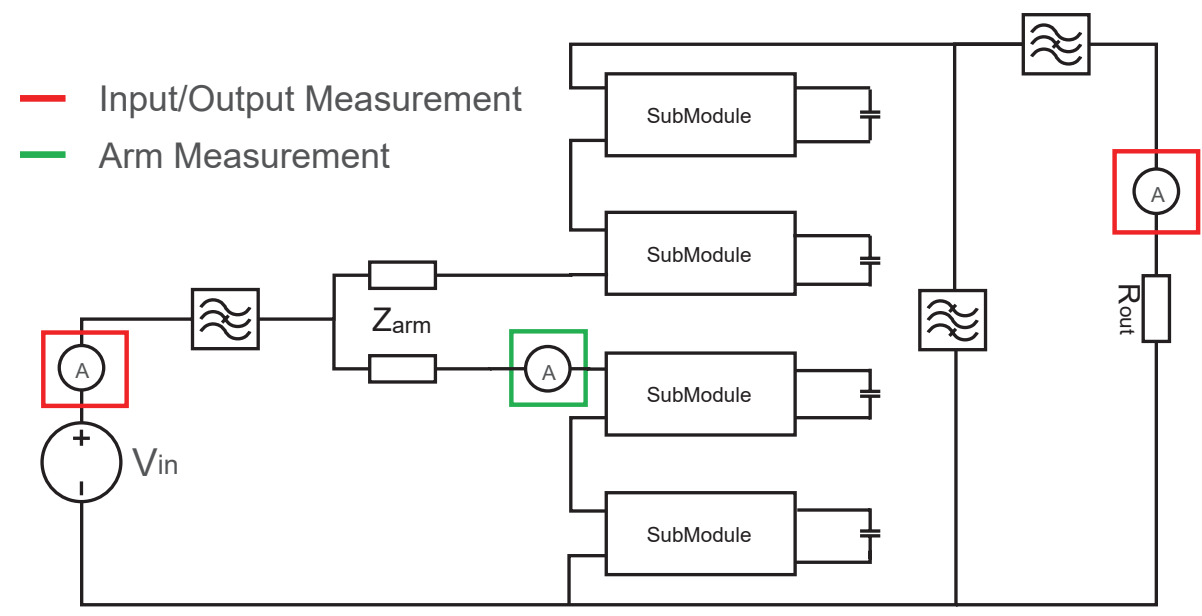

Figure 3.24: Measurement setups.

DC component is filtered. Expected are spectra that are similar to the ones shown in sections 3.2.3 and 3.2.5.

One important thing to notice, is that there are in total three connections to the mains. Each metallic box in Figure 3.25 has an AC/DC converter which either provides a $60 \mathrm{~V}$ or $30 \mathrm{~V}$ DC voltage. No Line Impedance Stabilization Network (LISN) has been added to the setup, however, no EMI from the mains was measured on the DC side of the converter. A spectral analysis of the source connected with a resistor of $47 \Omega$ showed an average noise of approx. $-90 \mathrm{dbA}$ from $20 \mathrm{~Hz}$ to $1.25 \mathrm{MHz}$.

\subsubsection{Results}

The mean of the spectra resulting from the measured currents are shown in Figure 3.26. In case of the I/O measurements the mean is taken over 8 measurements, while in the arm measurement it is taken over three measurements. Based on the analysis of section 3.2.3, the importance of the harmonics and subharmonics were identified. The secondary frequency $\left(f_{m}\right)$ and its harmonics $\left(H_{m}\right)$ are indicated with red vertical lines, while the switching frequency $\left(f_{c}\right)$ and its harmonics $\left(H_{c}\right)$ are indicated with green vertical lines.

Based on the analysis in sections 3.2.3 and 3.2.5, it was expected that the carrier and all even numbered harmonics (and sub-harmonics) were suppressed in the EMI spectrum. The result shown in Figure 3.26 is exactly as expected [52].

Interference produced by a single submodule was canceled by cascading multiple SMs in a single arm. The measurements have shown that harmonic cancellation occurs in a multi-level system based on the M3C topology, when the reference signals used in the gatedrive signal have a phase-offset. Based 


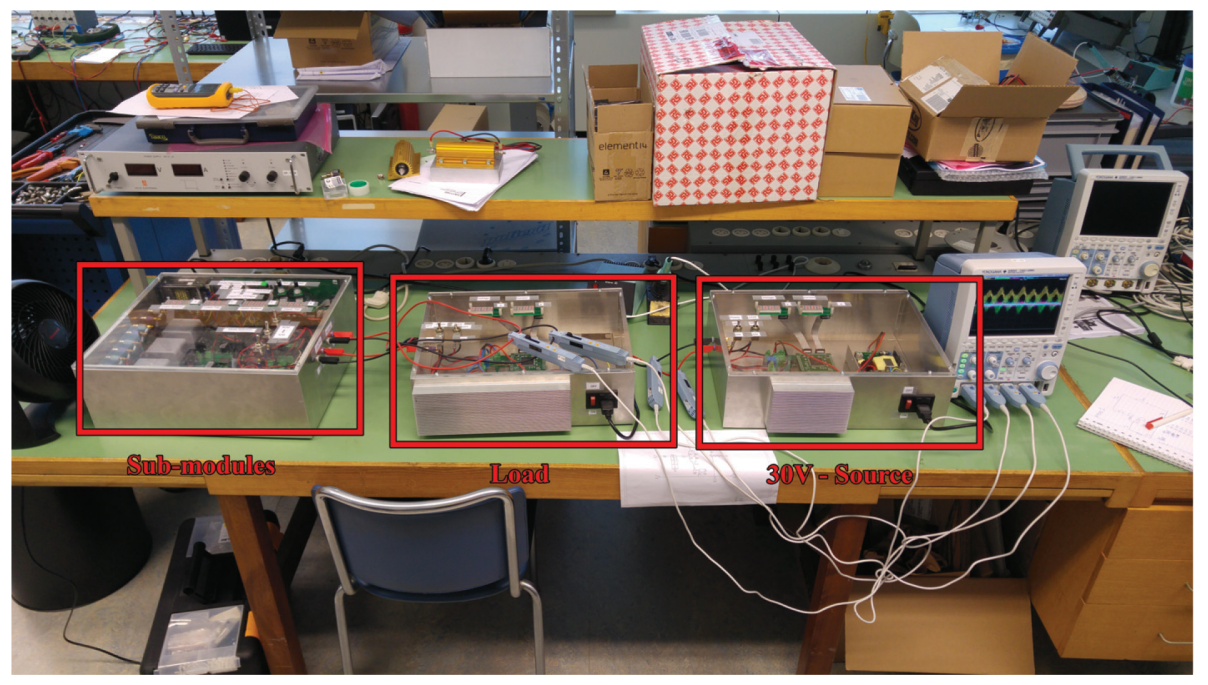

Figure 3.25: Measured currents in I/O measurements.

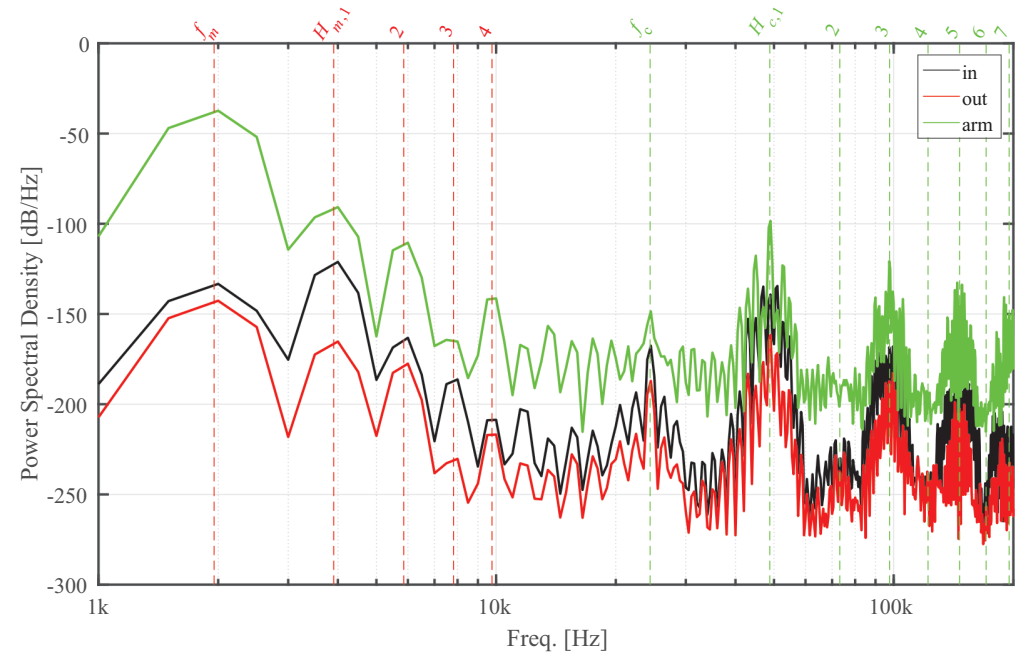

Figure 3.26: Mean of measured spectral densities of input, output and arm. 
on the theory, the effect of shifting the interference into higher frequencies increases with an increasing number of SMs being used in a single arm. Scaling the technology to high voltage DC networks should therefore be beneficial with respect to EMI emissions.

\subsection{Summary}

This chapter has described the development of a sPWM generator for use in a multilevel converter. Its development is aimed at flexibility for research into power systems at much higher frequencies than the conventional approached. Based on the measurements performed in this chapter it is expected to be able to make the system response less effective in creating emissions. As functional behavior is dependent of low frequency components. These are at least ten times smaller than the individual SM switching frequencies.

The modulation frequency is the functional frequency used within the converter system. The carrier frequency can be altered to shift the generated EMI spectrum. The control parameter AC-amplitude and DC-offset are directly related to functional behavior of the system, i.e. the system's transfer function is dependent on their setting. They have a direct impact on the generated EMI spectrum, however combined with the results of the theoretical model this can only be seen in the sub-harmonics of the carrier. Dead-time is inserted for practical reasons, to not create a DC fault inside individual half-bridge modules. Based on the measurement results, it has an increasing impact on power transfer at increased switching frequencies. i.e. increasing the dead-time will result in less power being transferred to the load (Figure 3.13). Increasing the number of SMs has shown to effectively canceling carrier frequencies. Basically shifting the zeroth harmonic of the switching frequency proportional to the number of modules.

Concluding this chapter, the possibility to make the system response less effective in creating emissions by playing with the parameters is identified. This way optimization for higher-frequency EMI becomes possible and will be studied in following chapters. In general, many power electronic researchers are not looking at the EMI potential, nor the system emission which can be considered the main contribution of this thesis. 


\section{Chapter 4}

\section{Filtering}

The previous chapters described the M3C and more specifically the noise generated inside such a system. Parameters influencing the generated EMI have been studied and identified. In general, the next step in designing a system will be related to containing or mitigating the noise. It has already been mentioned in the outline of this thesis, that in [15] a classification of conducted EMI mitigation techniques has been shown. Following Figure 1.6, two main streams were identified, mitigation at the source, and along the propagation path. As the first one has been partially addressed in chapter 2 and 3, the following chapter will focus on the latter. Also from Figure 3.24 it is clear that filters are an integral part of the converter design, and thus one of the key objectives of the project is optimizing filters.

Filter design is a research area of importance for EMC as these are commonly used for EMI mitigation in a wide range of applications, while their usage is especially relevant in power systems. With the increasing trend of miniaturization, modularization and higher switching speeds in power electronics, a need for accurate high frequency component models arises due to unexpected high frequency behavior [13].

This chapter is split into three major parts. The first part focuses on obtaining equivalent circuit component values based on impedance curves. The mathematical extraction method is generalized to equivalent circuits with a theoretically infinite amount of RLC components and any impedance curve. In the second part of the chapter, the full wave $3 \mathrm{D}$ modeling of passive components is discussed, with a big focus on the CMC as it is one of the most important (and most challenging) components to model accurately at higher frequencies. The last part is about integrating the obtained results from $3 \mathrm{D}$ field simulations into equivalent circuits and optimizing filter design using these tools.

Parts of Section 4.1, Section 4.1.2, Section 4.3 and Section 4.4 are published in [53], [54], [55] and [56] respectively. 


\subsection{Automated Equivalent Circuit Values}

In industry the optimization towards maximum profit never stops. The electronics industry is no exception, thus the produced devices need to become cheaper, better and smaller. Every inch of performance is being squeezed out of every single component, while compliance is met. The level of Engineering/Designing required is increasing as well. Simulation software is being used to verify designs in a cheap and fast way, thus less, costly prototyping is required. The most widely used tool for an electrical engineer remains an equivalent circuit simulator.

Since the used frequencies are increasing, while component sizes are reducing, accurate simulations tend to become harder. Circuit simulations based on ideal component values proved to be inadequate for EMI performance evaluation. Adding non-idealities of components, PCB-traces, wires etc. lead to a temporary solution. However, even this is showing inadequacy at the task at hand [13]. The investigation of parasitic effects of individual components has lead to simplistic models where physical origins were derived from, as is for instance explained in [57]. The equivalent circuits shown in [57] are displayed in Figure 4.1.

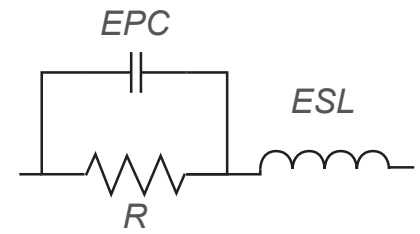

(a) Resistor

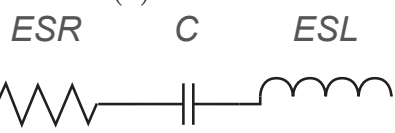

(c) Capacitor

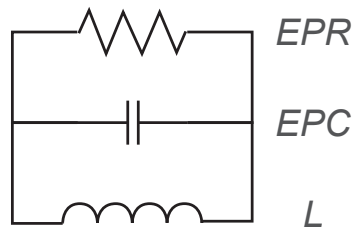

(b) Inductor

Figure 4.1: Equivalent circuit models of non-ideal components

In case of a non-ideal capacitor, the frequency dependent impedance can be modeled with an equivalent circuit consisting of a series connection between three ideal RLC-components. Where the inductance and resistance are considered to be a parasitic effect and named Equivalent Series Inductor (ESL) and Equivalent Series Resistor (ESR) respectively and shown in Figure 4.1c. In case of a non-ideal inductor, they are parallel connected as shown in Figure 4.1b, with the capacitance and resistance to be parasitic and named Equivalent Parallel Capacitor (EPC) and Equivalent Parallel Resistor (EPR) respectively. The origin of ESL is often explained through the leads of the capacitors, while ESR is the conductivity of the materials. EPC is often thought to be the sum of several capacitances, while EPR is the considered to be a turn to turn field effect. Their values can be determined via impedance measurements. 
The parasitic effects can be extended to higher complexity by incorporating mutual coupling effects between components, traces or even between component-parts. For relatively simple passive components like capacitors or inductors, inter-component coupling effects can be incorporated (based on curve-fitting) to increase circuit simulation accuracy $[56,58]$. The physical basis of these simplified models are explained in for instance [59]. For instance, the leads of a capacitor form an AC-current loop with produces a magnetic flux that is picked up by any other loop and can be considered an inductive coupling.

3D simulators are used to investigate these cases, to go where a circuit simulator cannot. Such full-wave solvers include the near-field coupling between components. Analysis and optimization strategies based on 3D simulations have been suggested in [60-63]. However, it requires expert knowledge and experience to create an accurate 3D simulation. Alternatives for 3D modeling to acquire the mutual coupling impedance $\left(Z_{m c}\right)$ do exist. In case of wires, analytical expressions for predicting the amount of crosstalk are actively being improved [64-66]. It was shown in [67] that from measuring scattering parameters between different components, one can determine mutual coupling effects and add these to the equivalent circuit simulation. From this point on G. Asmanis et al. has shown that it is possible to determine the mutual coupling through 3D simulations using simplified models [68]. As has been suggested in $[58,69]$, one can combine both worlds into a method, which can be applicable to for instance filter design, digital PCB or power electronics. Here it is suggested to further extend the research and incorporate an automated equivalent circuit extraction for self-parasitics as well as for mutual-parasitics.

This section focuses on the automated extraction of circuit model parameters using a Gauss-Newton algorithm for the purpose of back-annotating this into a system equivalent circuit simulation. The main problem to be addressed is the accurate representation of impedances into equivalent circuits. Impedance is used here in broadest sense possible, since it can be either the measured, simulated (3D) or even calculated impedance of any phenomenon. The optimization is a general method for finding a possible equivalent circuit that represents the impedance in passive elements. The method is applied to multiple cases. In the following section 4.1.1, the algorithm is explained in a mathematical form, showing that there is no limit to the amount of parameters to be used. In section 4.1.4 the method is applied to the design of a filter. The possible couplings between components will be discussed and inserted into the equivalent circuit simulation.

\subsubsection{Optimization problem}

This section describes the optimization procedure which is used to find appropriate equivalent circuit representations. It will be described in general form, to show its applicability to equivalent circuits with an arbitrary number of elements. Assume that the impedance of a certain coupling phenomena that 
has to be simulated is known, either by measurement, 3D simulation or any other method. The impedance of an equivalent circuit can then be matched to this impedance data in the frequency domain by performing an optimization. Therefore, a representation of the impedance of this equivalent circuit in terms of its passive elements (inductors, capacitances and resistances) is required. Assume that this has the following form:

$$
Z(f, \mathbf{x})=g\left(f, C_{1}, \ldots, C_{i}, L_{1}, \ldots, L_{j}, R_{1}, \ldots, R_{k}\right)
$$

which contains in total $p$ elements, consisting of $i$ capacitors, $j$ inductors and $k$ resistors. The aim is to find optimal values for these $p$ elements, which are contained as entries in the $p$-dimensional vector $\mathbf{x}$.

Define a vector containing the absolute value of the evaluation of this function at different frequency values $f_{1}, \ldots, f_{n}$ :

$$
\mathbf{Z}=\left(\begin{array}{c}
\left|Z\left(f_{1}, \mathbf{x}\right)\right| \\
\vdots \\
\left|Z\left(f_{n}, \mathbf{x}\right)\right|
\end{array}\right)
$$

Denote the simulated or measured impedance data in absolute value as $\mathbf{Z}_{m c}$, which is also a vector containing entries for the same frequencies. Then the optimization objective is to find values for all inductors, capacitors and resistors in the equivalent circuit, such that the impedance values in (4.2) match the values in $\mathbf{Z}_{m}$. To obtain a least-squares fit the following objective function should be minimized:

$$
h(\mathbf{x})=\|\mathbf{r}(\mathbf{x})\|_{2}^{2}=\left\|\mathbf{Z}-\mathbf{Z}_{m}\right\|_{2}^{2}
$$

Here $\|\cdot\|_{2}$ represents Euclidean distance, and $\mathbf{r}(\mathbf{x})$ is the vector of residues when the values of $\mathbf{Z}$ are compared to $\mathbf{Z}_{m}$.

An overdetermined objective function (assuming $n>p$, meaning more frequency points than passive elements) such as (4.3) can be minimized by the iterative Gauss-Newton method. Starting from an initial $\mathbf{x}_{0}$, iteration steps are defined by:

$$
\mathbf{x}_{n+1}=\mathbf{x}_{n}+\left(\mathbf{J}_{\mathbf{r}}^{T} \mathbf{J}_{\mathbf{r}}\right)^{-1} \mathbf{J}_{\mathbf{r}}^{T} \mathbf{r}\left(\mathbf{x}_{n}\right)
$$

Here the superscript $T$ denotes transpose and the $n$ by $p$ Jacobian matrix of the residues $\mathbf{J}_{\mathbf{r}}$ contains the following entries:

$$
\left(\mathbf{J}_{\mathbf{r}}\right)_{i j}=\frac{\partial r_{i}}{\partial x_{j}}\left(\mathbf{x}_{n}\right)
$$

Here $r_{i}$ and $x_{j}$ are the $i^{t h}$ and $j^{t h}$ entries of the vectors $\mathbf{r}$ and $\mathbf{x}$, respectively. Stopping criterion of the iteration is usually a threshold value $\delta$ for the norm given in (4.3).

To avoid divergence and improve convergence, the Levenberg-Marquardt extension of the Gauss-Newton algorithm is applied. In this case, the update 
step in (4.4) changes in:

$$
\mathbf{x}_{n+1}=\mathbf{x}_{n}+\left(\mathbf{J}_{\mathbf{r}}^{T} \mathbf{J}_{\mathbf{r}}+\lambda \mathbf{D}\right)^{-1} \mathbf{J}_{\mathbf{r}}^{T} \mathbf{r}\left(\mathbf{x}_{n}\right)
$$

in which $\mathbf{D}$ is the diagonal matrix containing the diagonal elements of $\left(\mathbf{J}_{\mathbf{r}}^{T} \mathbf{J}_{\mathbf{r}}\right)$. The parameter $\lambda$ is the Marquardt parameter. When it equals zero, the iteration reduces to that of the Gauss-Newton algorithm. However, when with $\lambda=0$ the residue with $x_{n+1}$ is larger then that with $x_{n}$ (so with the new iteration step the residue is increased), then $\lambda>0$ should be set and the iteration step repeated. The value for lambda could be calculated by a line search, but since this implies an extra optimization for each update step, this is less efficient then simply increasing $\lambda$ until it results in an update step that reduces the residue. From there, if possible, lambda could again be reduced until it reaches zero.

An essential and sometimes challenging aspect of the Gauss-Newton optimization is acquiring an initial guess $\mathbf{x}_{0}$. This can be obtained by an educated guess based on knowledge of the system to be modeled. However, based on this knowledge some constraints can usually be imposed, such as:

$$
\begin{gathered}
C_{1, \text { low }} \leq C_{1} \leq C_{1, \text { up }} \\
\vdots \\
C_{i, \text { low } \leq} \leq C_{i} \leq C_{i, \text { up }} \\
L_{1, \text { low }} \leq L_{1} \leq L_{1, \text { up }} \\
\vdots \\
L_{j, \text { low } \leq} L_{j} \leq L_{j, \text { up }} \\
R_{1, \text { low }} \leq R_{1} \leq R_{1, \text { up }} \\
\\
\vdots \\
R_{k, \text { low } \leq} \leq R_{k} \leq R_{k, \text { up }}
\end{gathered}
$$

In view of an automated extraction of the equivalent circuit these constraints can be utilized. Together they form the solution space in which the optimal values of the passive elements can be found. An initial guess can then be obtained by performing a coarse search through this solution space, i.e. taking a logarithmic uniform grid between the lower and upper bound of each parameter, calculating the corresponding residues of each combination. Then $\mathbf{x}_{0}$ is taken to be the set of values that yields the lowest residue. From there on, the above iteration can lead to an optimal value for $\mathbf{x}$. The above procedure has been implemented in MATLAB, and in the following sections this optimization algorithm is applied.

\subsubsection{Components}

The previous section has showed a general method for determining the values of RLC components present in any equivalent circuit that can represent 
a certain frequency dependent impedance. Increasing the amount of parameters only increases complexity of the optimization and therefore computational time. In this thesis, the number of equivalent circuit components per physical component is limited to three. In other words, capacitors, inductors, CMCs, resistors, field-couplings etc. are all represented with three equivalent components. This implies that the fitting will be accurate only at the lower frequency range, which means up to or just beyond the first resonance. The technique developed will be applied to a Common Mode (CM) filter shown in Figure 4.2.

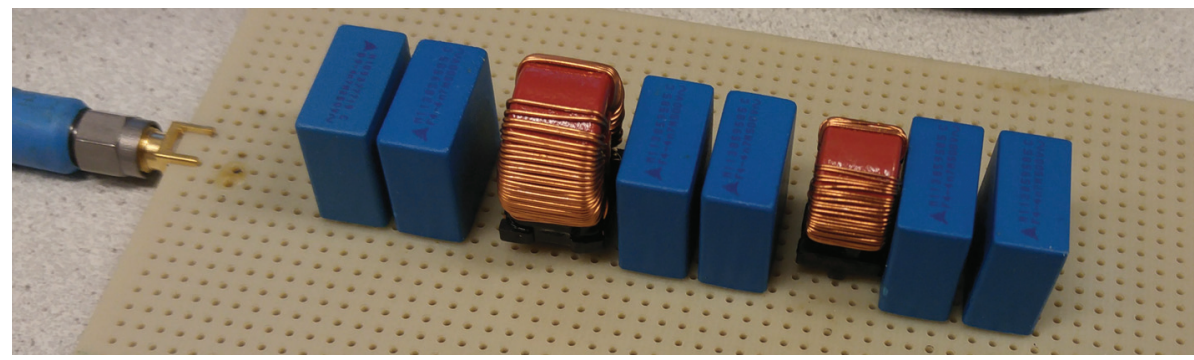

Figure 4.2: Measured filter, its schematic is shown in Figure 4.6a

It consists of eight physical components of which three are unique, with four unique combinations of field couplings. The components and mutual couplings are listed below:

1. C. Capacitor (TDK - MKP B81123)

2. L1. Common mode choke (WE744823210)

3. L2. Common mode choke (WE744823110)

4. Mutual Coupling parallel $\mathrm{C}$ and $\mathrm{C}$

5. Mutual Coupling parallel L1 and C

6. Mutual Coupling parallel L2 and C

7. Mutual Coupling parallel L1 and L2

The non-ideal behavior of inductors and capacitors has been studied extensively in the past. The self-parasitics of these components are mainly related to the geometry and determine their behavior at high frequency. As [63] mentioned, the EPC, EPR, ESL and ESR are well known and described in literature. Therefore, their explanation is omitted here, while the focus remains on recreating impedance curves with passive elements only. The CMC much more complex, and sections 4.2 and 4.3 .1 will be dedicated to modeling this component, however here the CM impedance of the chokes are modeled as the non-ideal inductors shown in Figure 4.1b. 
For the given components, the frequency dependent impedance data used for the optimization is obtained by measurement. The measurement device that was used is an impedance analyzer (Keysight ENA 5061B). An illustration of the measurement is shown in Figure 4.3a.

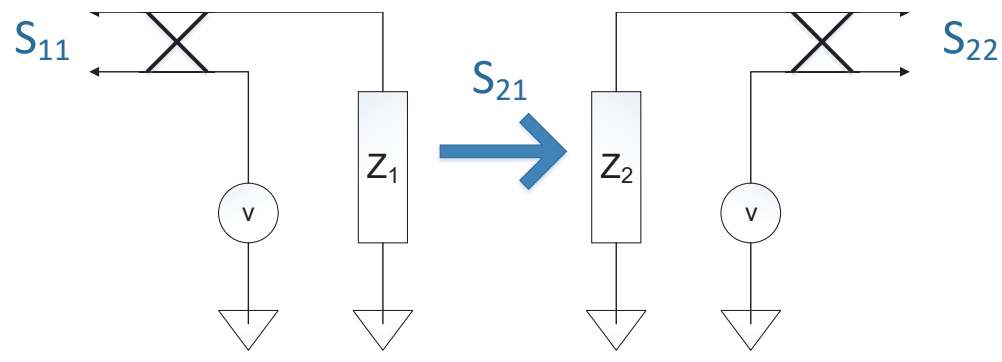

(a) S-parameter measurement using a Vector Network Analyzer (VNA) with two individual components connected that are not interconnected.

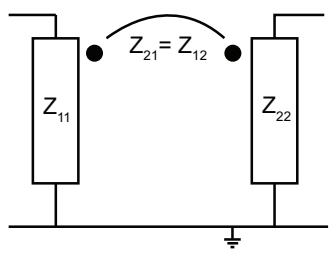

(b) Schematic representation of coupling between impedances that results from the standard S- to Z-parameter transform.

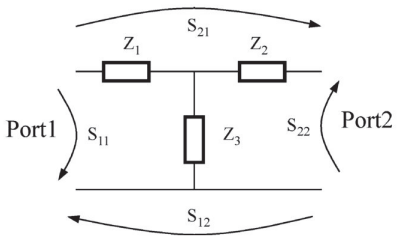

(c) Equivalent circuit for Zparameters of a reciprocal twoport network, adopted from [67].

Figure 4.3: Impedance measurement using a VNA.

In this section, seven impedances will be fitted to separate equivalent circuits as shown in Figure 4.1b and Figure 4.1c. Based on the standard Sto Z-parameter transformations with the assumption that the characteristic impedance $\left(Z_{0}\right)$ is $50 \Omega$, which holds for the Keysight ENA 5061B, three impedances can be derived from a single S-parameter measurement as has been shown in $[67]$ :

$$
\begin{array}{cc}
Z_{1}= & 50 \cdot \frac{1-S_{22}-S_{22} S_{11}+S_{11}-2 S_{21}+S_{21}^{2}}{\left(1-S_{22}+S_{22} \cdot S_{11}-S_{11}-S_{21}^{2}\right)} \\
Z_{2}= & 50 \cdot \frac{1+S_{22}-S_{22} S_{11}-S_{11}-2 S_{21}+S_{21}^{2}}{\left(1-S_{22}+S_{22} \cdot S_{11}-S_{11}-S_{21}^{2}\right)} \\
Z_{m c}= & 2 \cdot 50 \cdot \frac{S_{21}}{\left(1-S_{22}+S_{22} \cdot S_{11}-S_{11}-S_{21}^{2}\right)}
\end{array}
$$

By performing the S-parameter measurement as shown in Figure 4.3a and transforming them into Z-parameters, the results would represent the schematic 
as shown in Figure 4.3b. To incorporate these results in an equivalent circuit simulator like LTSPICE, one needs to convert this coupling either to the coupling factor, similar as with a transformer. However, [67] showed this is equivalent to a T-network of impedances, which can be seen in Figure 4.3c. Combining these steps, results in the Equations 4.8 to 4.10. Note that the resulting impedances are complex, while in the fitting algorithm the magnitudes are used. Also $\left(Z_{m c}\right)$ in Figure 4.5 should not to be confused with common mode impedance $\left(Z_{c m}\right)$ which is shown in Figure 4.4 .

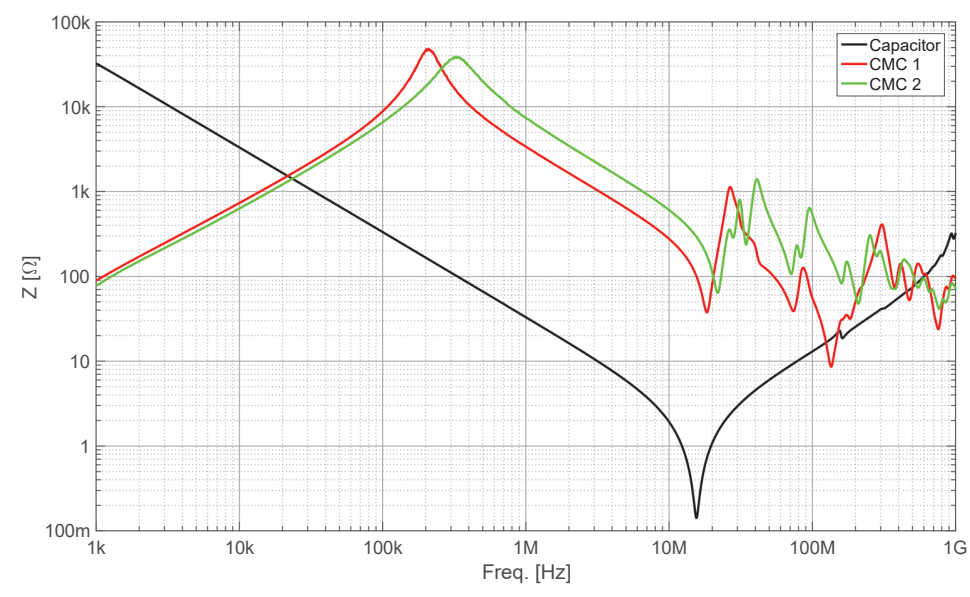

Figure 4.4: Impedance of used components, individual curves can be seen in Figure 4.8.

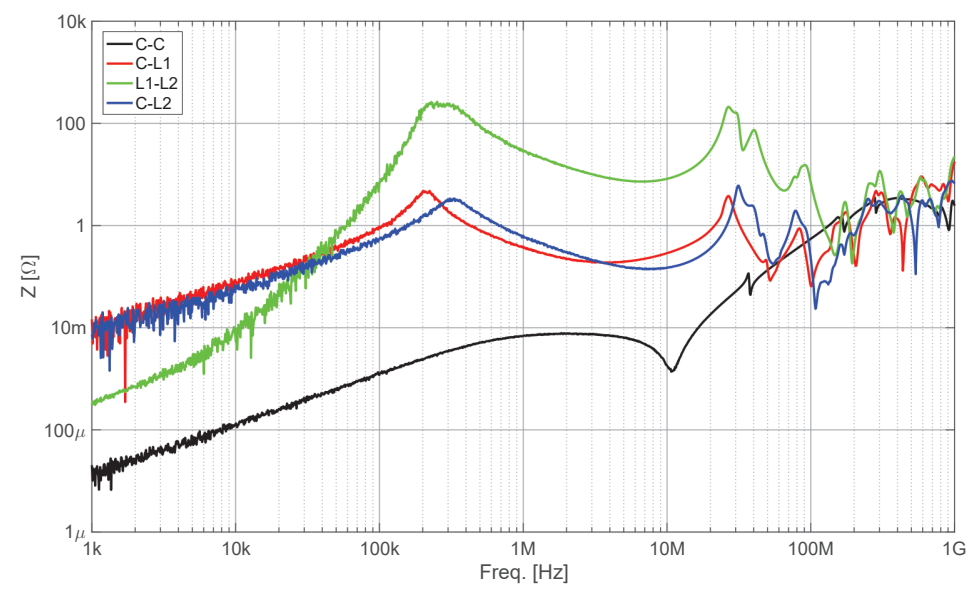

Figure 4.5: Mutual Coupling Impedance, individual curves can be seen in Figure 4.9 . 


\subsubsection{Impedance Fitting}

The measured components and their mutual couplings are used in a common mode filter as shown in Figure 4.2. It is a C-L-C-L-C filter, that exhibits numerous combinations with mutual coupling: 3 times $\mathrm{C}-\mathrm{C}$ coupling, 4 times L-C coupling and a single L-L coupling. However, the L1 and L2 are spatially separated and therefore their coupling can be neglected.

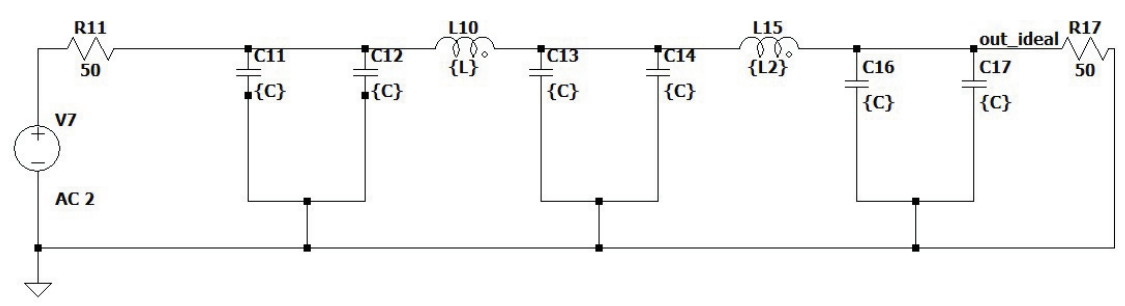

(a) Ideal Component simulation

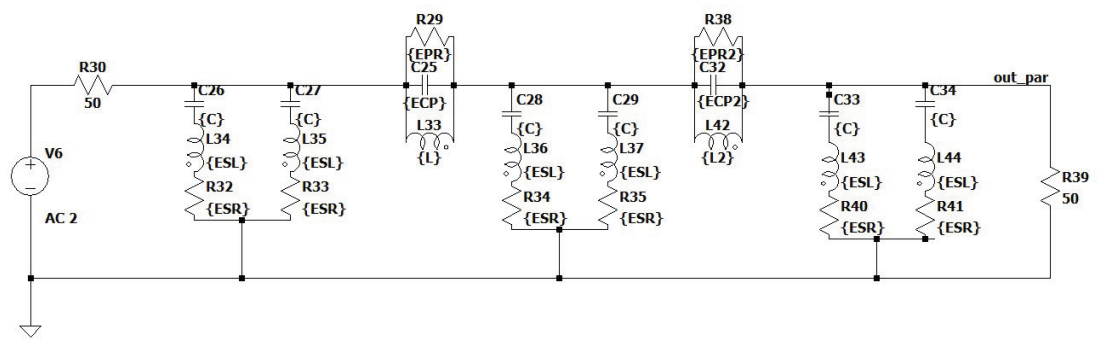

(b) Self-parasitic Component simulation

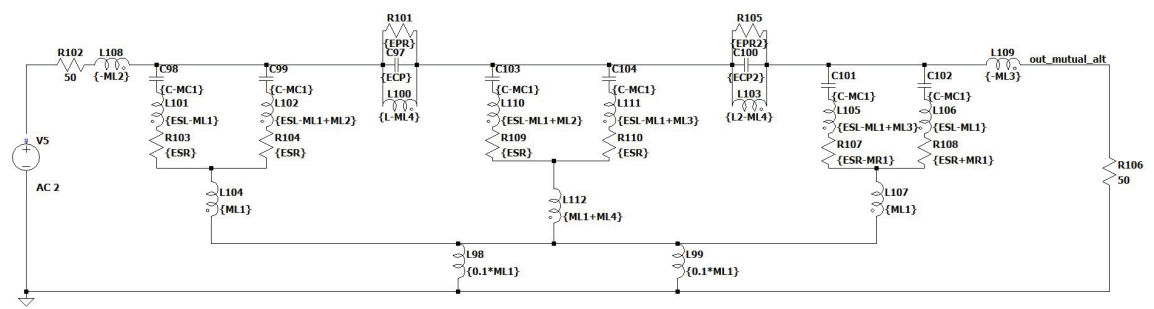

(c) Self-parasitic and Mutual Coupling Component simulation

Figure 4.6: LTSPICE Filter Schematics.

The impedance measurements as shown in Figure 4.4 and Figure 4.5 are fitted to the equivalent circuits in Figures $4.1 \mathrm{~b}$ and 4.1c. Their individual fitting results are shown in Figure 4.8 and Figure 4.9. The original measurements are shown in black, while the results from the initial guess (as described in section 4.1.1) are shown in red and the fitted results in green. The resulting RLC values are displayed in Table 4.1. 


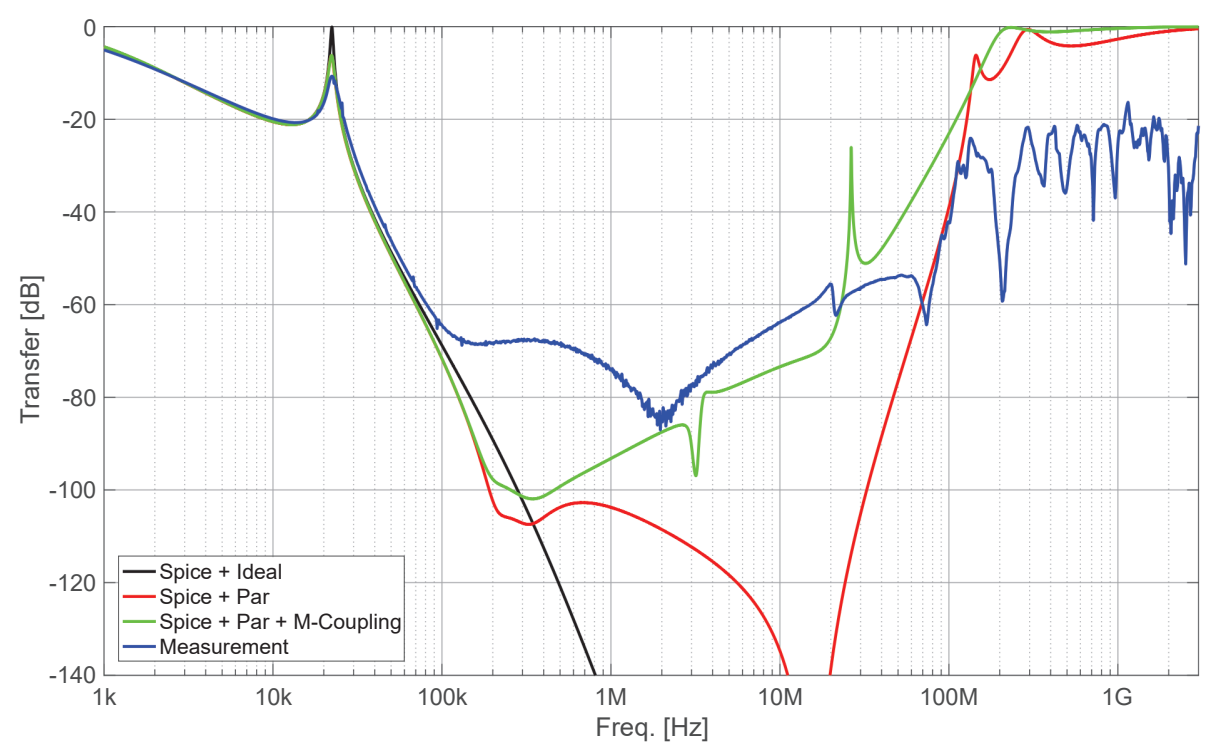

Figure 4.7: Comparison of LTSPICE simulations with the actual filter measurement.

It can be seen that the components are fitted nicely by the optimization algorithm. However, deviations occur when fitting the coupling impedances. Up until approximately $10 \mathrm{MHz}$ the fitting of C-C and C-L are fairly accurate. However the fit of the extracted mutual impedance between the two CMCs holds only between $200 \mathrm{kHz}$ and $1 \mathrm{MHz}$. The coupling does not behave linearly with increasing frequency, hence the complexity of equivalent circuit that is fitted should be increased. In the following section the filter is simulated using LTSPICE and compared to a measurement using a VNA.

\subsubsection{Filter}

The filter has been simulated with LTSPICE in three different configurations. First, only the ideal component values were used (Figure 4.6a), then the selfparasitics of the CMC and capacitors are added (Figure 4.6b) and at the third instance the mutual inductances are added (Figure 4.6c) according to the method given in [67] and further clarified in section4.4. Note however that in Figure 4.6c, the lowest two inductances are based on the coupling between the capacitor stages. It is here assumed that only $10 \%$ of the coupling between capacitors in a single stage is remaining due to the increased distance. In Figure 4.7 the three simulations are compared to measurement results. In case of the ideal simulation, it is approximately valid up to $100 \mathrm{kHz}$. Adding self-parasitics will increase accuracy from this point, however one needs to also incorporate the mutual parasitics as they tend to play a role in the same fre- 


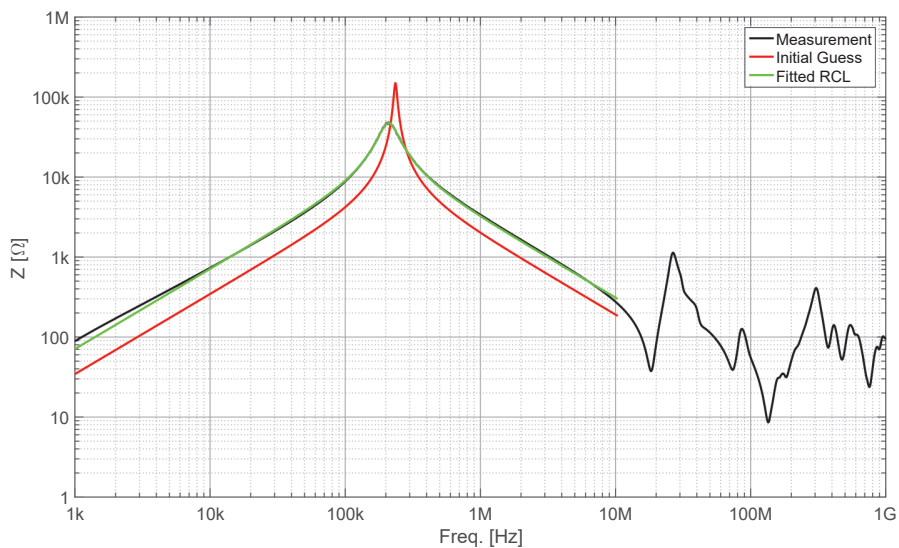

(a) L1 - CMC WE744823210

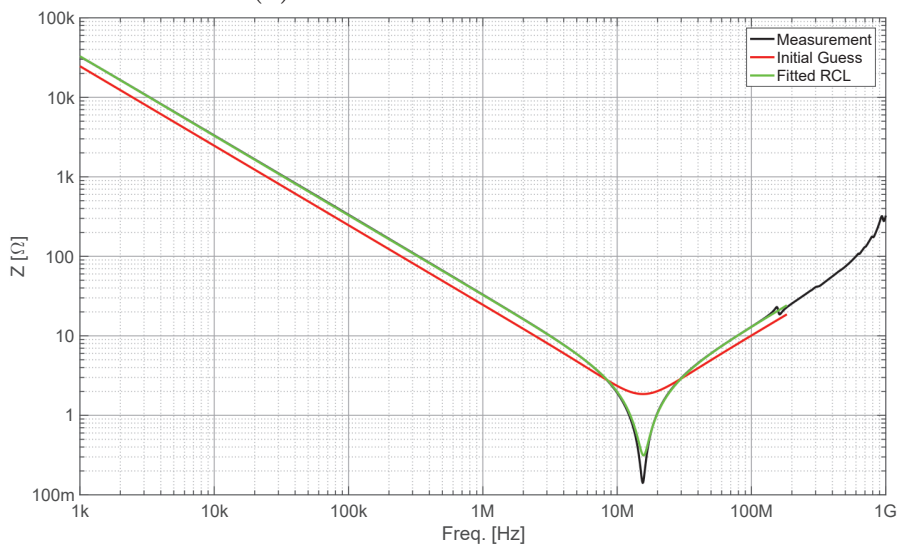

(b) Capacitor - MKP B81123

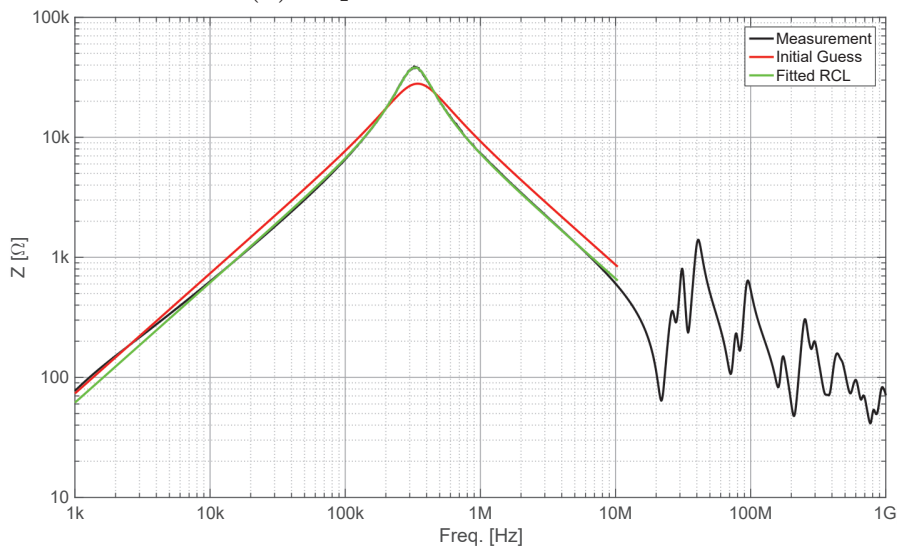

(c) L2 - CMC WE744823110

Figure 4.8: Fitting applied to multiple components. In all cases the curves are representing Measurement (black), Initial Estimation (red) and Fitted Curve (green). 

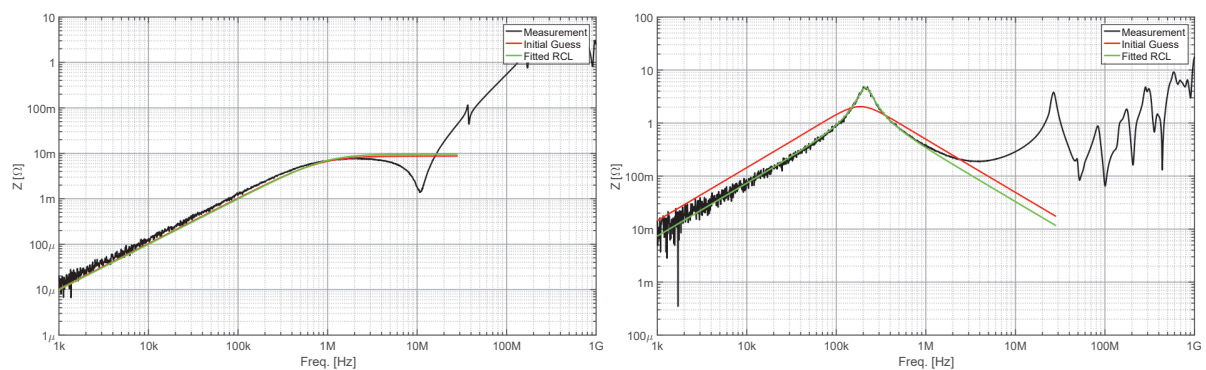

(a) Mutual Impedance between parallel (b) Mutual Impedance between parallel placed capacitors)

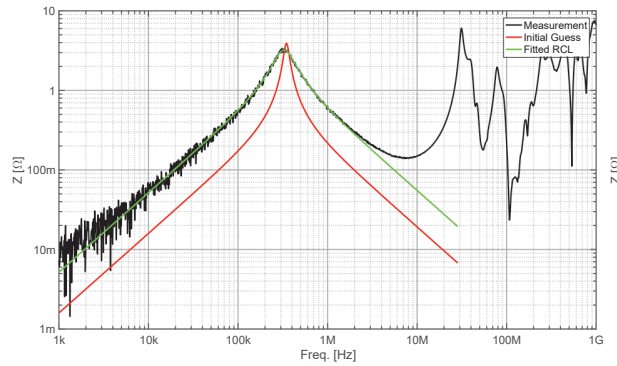
placed $\mathrm{C}$ and $\mathrm{L} 1$

(c) Mutual Impedance between parallel (d) Mutual Impedance between parallel placed $\mathrm{C}$ and $\mathrm{L} 2$ placed L1 and L2

Figure 4.9: Fitting applied to multiple mutual coupling impedances. In all cases the curves are representing Measurement (black), Initial Estimation (red) and Fitted Curve (green).

Table 4.1: Fitted parameter values and time it took for the initial guess $\left(t_{\text {init }}\right)$ and optimization $\left(t_{o p t}\right)$ to complete, with the amount of optimization iterations $(N)$.

\begin{tabular}{|l||c|c|c|c|c|c|c|}
\hline Type & Circuit & $\mathrm{R}$ & $\mathrm{L}$ & $\mathrm{C}$ & $t_{\text {init }}$ & $t_{\text {opt }}$ & $\mathrm{N}$ \\
\hline Capacitor & series & $313.2 \mathrm{~m} \Omega$ & $21.1 \mathrm{nH}$ & $4.9 \mathrm{nF}$ & $3.6 \mathrm{~s}$ & $129 \mathrm{~ms}$ & 96 \\
\hline CMC1 & parallel & $47.5 \mathrm{k} \Omega$ & $11.3 \mathrm{mH}$ & $51.1 \mathrm{pF}$ & $2.6 \mathrm{~s}$ & $5.7 \mathrm{~ms}$ & 10 \\
\hline CMC2 & parallel & $38.1 \mathrm{k} \Omega$ & $9.8 \mathrm{mH}$ & $23.9 \mathrm{pF}$ & $2.6 \mathrm{~s}$ & $5.8 \mathrm{~ms}$ & 9 \\
\hline C//C & parallel & $9.7 \mathrm{~m} \Omega$ & $1.6 \mathrm{nH}$ & $2.9 \mathrm{fF}$ & $2.2 \mathrm{~s}$ & $1.8 \mathrm{~s}$ & $4.1 \mathrm{k}$ \\
\hline L1//C & parallel & $4.6 \Omega$ & $1.2 \mu \mathrm{H}$ & $482 \mathrm{nF}$ & $2.9 \mathrm{~s}$ & $16 \mathrm{~ms}$ & 8 \\
\hline L2//C & parallel & $3.2 \Omega$ & $824 \mathrm{nH}$ & $288 \mathrm{nF}$ & $2.1 \mathrm{~s}$ & $10 \mathrm{~ms}$ & 16 \\
\hline L1//L2 & parallel & $262 \Omega$ & $59.8 \mu \mathrm{H}$ & $5.4 \mathrm{nF}$ & $2.2 \mathrm{~s}$ & $18 \mathrm{~ms}$ & 36 \\
\hline
\end{tabular}


quency bandwidth.

In this study, an algorithm has been implemented to automate the extraction of passive component values from measured impedance curves. Even though the method is not limited to only measurements of components, it has been applied to a CM filter and its components. The self-parasitic values were extracted and their mutual coupling impedance was represented in an equivalent RLC-circuit. The assumption was made that a simple three parameter RLC-circuit is an accurate representation of this coupling, however the results have shown that there is some deviation. For the mutual coupling between two capacitors and, capacitor and inductor, the fitted results hold up to approximately $1 \mathrm{MHz}$. However for CMC to CMC coupling, the deviation is large and different equivalent circuits should be sought. In general, it can be concluded that the fitting algorithm works, however more complex equivalent circuits need to be added to increase flexibility in fitting all forms of impedance curves accurately. Note that in our implemented case a good fit will only occur when the slopes are $20 \mathrm{~dB} /$ decade, due to the fact that the $\mathrm{C}$ and $\mathrm{L}$ in Figure 4.1 are linearly related to frequency. To conclude, the algorithm has shown the possibility to automatically back-annotate parasitics and mutual inductances into circuit simulators.

\subsection{CMC - Equivalent Circuit Modeling}

For more complex components, such as the CMC, the self parasitics are already a challenge to address [70,71]. Not only a mutual coupling exists within the component itself, but it is dependent on the type of current (CM/DM). Also non-linear behavior of the magnetic core material contributes to the complexity of modeling the component accurately [72]. As power electronic components are switching faster, i.e. rise and fall time, and more often per second, it becomes important to include the real physical behavior of filter components and especially the CMC. Here in this section the CMC is introduced through equivalent circuit modeling with a focus on the capacitive behavior occurring above the first resonance. In section 4.3 field effects are taking into account through full 3D modeling of a CMC.

Many models exist for describing a choke's behavior [70-76]. In Figure 4.10 three models are shown that were adopted from the literature mentioned before. They all show similarities with the equivalent impedance of a non-ideal inductor shown in Figure 4.1b.

Sometimes the characteristics of the core are taken into account, but often only by including the mutual inductive coupling or frequency dependent resistance (Figure 4.10c). Based on the equivalent circuit for three parallel and (magnetically) coupled inductors (Figure 4.10c) one should be able to predict the approximate CM impedance based on a measurement of a single phase.

In $[73,74]$ it is assumed that the inductive coupling has negligible, to no, effect on the capacitive behavior of the CMC. Based on the explanation of the physical origin and analytical calculation of the capacitive behavior from [70] 


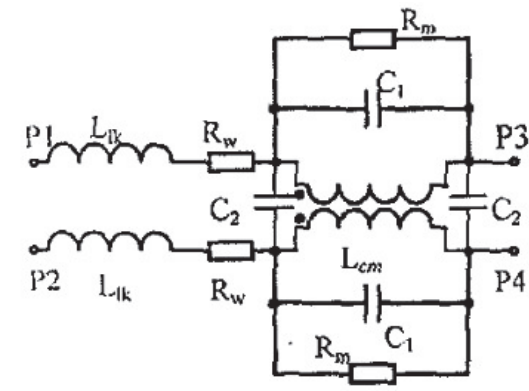

(a) Adopted from [73]

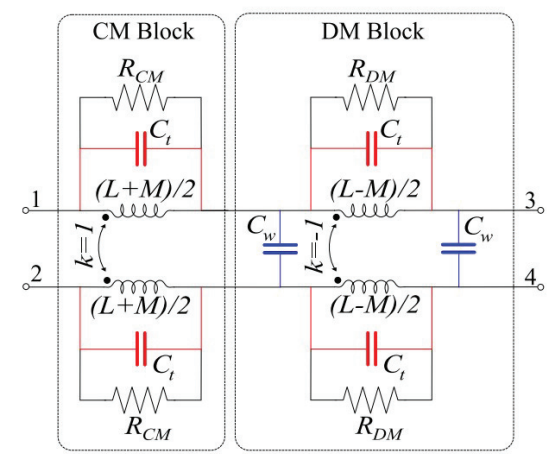

(b) Adopted from [76]

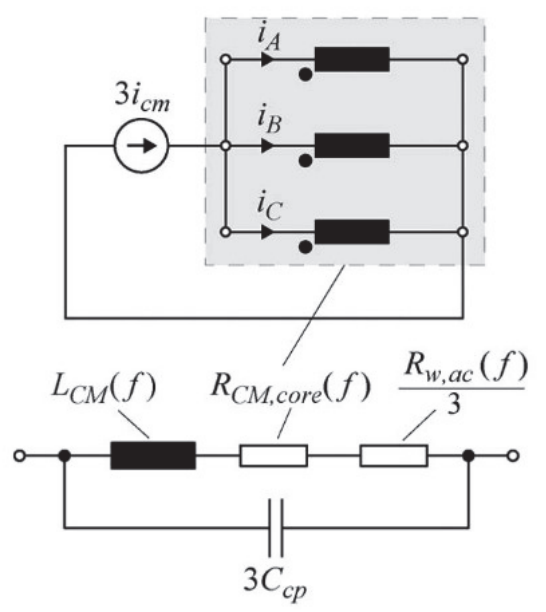

(c) Adopted from [74]

Figure 4.10: Equivalent circuit models of common mode chokes. 
and [77], this assumption seems to be a valid one. In the calculation, it is assumed the capacitance is an infinite sum of small contributing capacitances. Integrating them all (or most of them at least) the surface integral will result in approximately the total capacitance. Their results show good agreement between measurements and calculations.

Equivalent circuit modeling based on curve fitting is a useful tool for describing a component or systems behavior in terms of circuit elements. In many cases it has been shown that the resulting equivalent circuit has a physical origin and can thus be explained by physical parameters. This section focuses on the modeling of the equivalent parasitic capacitance of a $\mathrm{CMC}$ and its physical origin.

In section 4.2.1 it is shown that the curve fitting method is not suited as a basis for deriving a physics based model. Section 4.2.2 increases the contribution of turn-to-turn capacitance $\left(C_{t t}\right)$. In section 4.2 .3 the effect of coupling through the magnetic core is investigated by measurements, which shows a need for incorporating inductive behavior in modeling a CMC at higher frequencies.

\subsubsection{Modeling through Curve Fitting}

In Figure 4.1b a possible equivalent circuit for a single inductor was introduced and Figure 4.10c shows that by placing three magnetically coupled impedances in parallel the CMC could be modeled. From the impedance measurement of a single phase $\left(Z_{\text {phase }}\right)$, also known as line impedance, the RLC-values of the non-ideal inductor circuit can be extracted. Putting three of these circuits in parallel, with the addition of the mutual coupling, should result in the CM-impedance $\left(Z_{c m}\right)$. Using the following equations, one could derive the appropriate values of the RLC-circuit and model the $Z_{\text {phase }}$ and $Z_{c m}$. The coupling coefficient $k$ is often considered to be equal to 1 .

$$
\begin{gathered}
L=\frac{\left|Z_{x x}\right|}{2 \pi f} \\
E P C=\frac{1}{2 \pi f \cdot\left|Z_{x x}\right|} \\
E P R=\max \left(Z_{x x}\right) \text { at } f_{L=E P C} \\
M=k \cdot \sqrt{L_{1} L_{2}}
\end{gathered}
$$

In Equations 4.11 to $4.13 Z_{x x}$ can be either $Z_{\text {phase }}$ and $Z_{c m}$. Using Equation 4.11 the ideal inductance value can be calculated from the impedance under consideration, while from Equation 4.12 the ideal capacitance value can be derived. Note however that the impedance should be evaluated before and after the resonance peak respectively with these equations in the areas of where the magnitude of the slope is $20 \mathrm{~dB} / \mathrm{dec}$. The ideal capacitance is in case of an inductance a parasitic one, which in the equivalent circuit is denoted as EPC. Equation 4.13 is used to calculate the Equivalent Parallel Resistor. In case of 
the equivalent circuit presented in Figure 4.1b this is where the impedance is at a maximum, in which the magnitude of the reactances of $L$ and $E P C$ are equal but opposite in phase, i.e. the resonance peak of the impedance.

The resulting fitted line, which represents the $Z_{\text {phase }}$ as it was derived from the line impedance measurement, is then compared with the CM impedance measurement. Since this shows a good agreement, one would assume the modeling has been done correctly in this case and physical origins for the equivalent circuit components can be sought.

In $[77,78]$ a combination of numerous of capacitive effects results in the total EPC. They consist of, among others, Turn-to-Turn $\left(C_{t t}\right)$, Turn-to-Core $\left(C_{t c}\right)$ and Turn-to-Ambient capacitance $\left(C_{t a}\right)$. The simplest models only incorporate the $C_{t t}$. The measured EPC of a single phase should therefore be twice as small as the measured parasitic capacitance from the CMC. Due to the mere fact, that only half the amount of windings are being measured and that they are placed in parallel. It was demonstrated for a single CMC, that the derived capacitance of a single phase is equal to the measured capacitance of two parallel phases.

In the following section, an investigation on the parasitic capacitance is performed. Another approach is proposed to address the physical origin of the equivalent 'capacitive' behavior of CMCs and inductors in general.

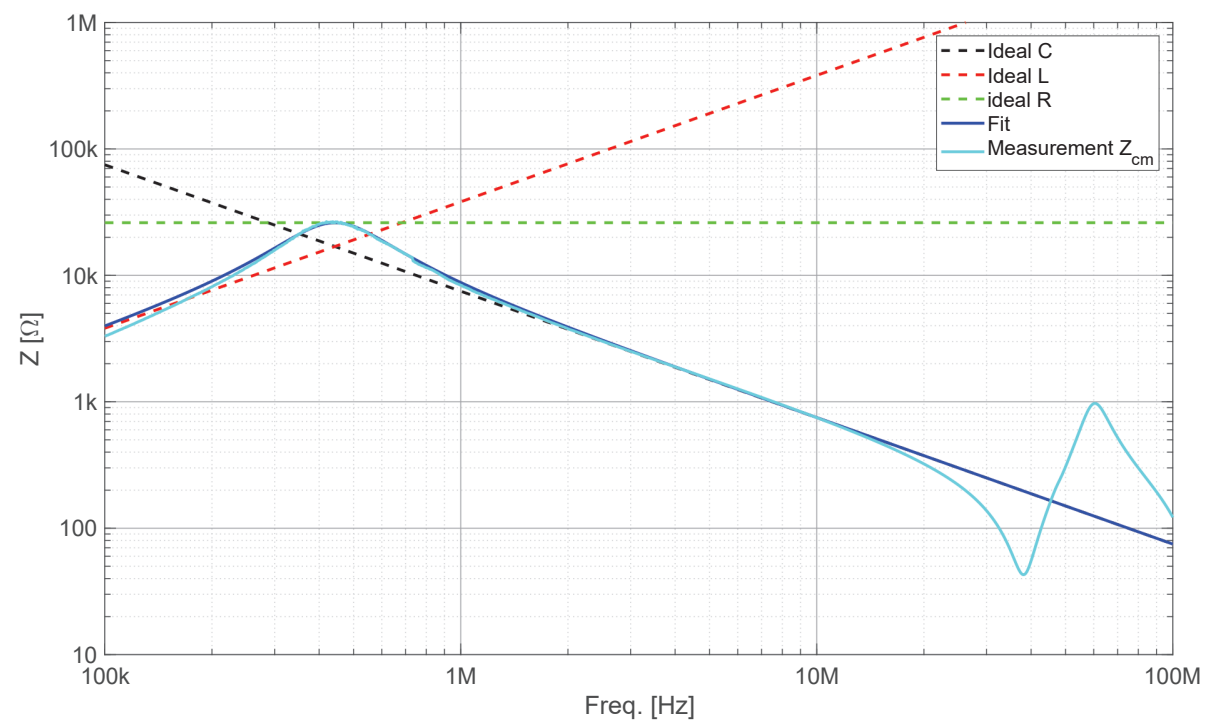

Figure 4.11: Equivalent circuit (Figure 4.1b) fitting based on the $Z_{\text {phase }}$ measurement with $\mathrm{N}=26$ compared to a $Z_{c m}$ measurement. $Z_{c m} \neq Z_{\text {phase }}$ is expected. 


\subsubsection{Towards a Physics Based Approach}

Several two-phase (Figure 4.12a) and three-phase (Figure 4.12b) common mode chokes have been measured. A choke can be considered as two (or three) inductors on the same core, that introduces a mutual coupling effect through magnetic flux. The modeling of their non-ideal behavior has been addressed in section 4.2.1 and shown in Figure 4.1b. The investigated EPC is considered to arise from a combination of multiple physical phenomena. The most obvious ones have been addressed in numerous publications, which are mostly capacitive in origin. $C_{t t}, C_{t c}$ and $C_{t a}$ are considered to be the dominant effects in most cases. In case the combined contribution of these capacitances are relatively small compared to some other unknown one, the assumption that they are dominant might be inaccurate. This section is divided into two subsections. Section 4.2.2 shows the phase and CM measurements of three different chokes. By increasing the windings it is assumed the EPC increases and the summed capacitive phenomena becomes the dominant effect. In section 4.2 .3 the effect of mutual (inductive) coupling is investigated, by removing and introducing coupling in several measurement setups.

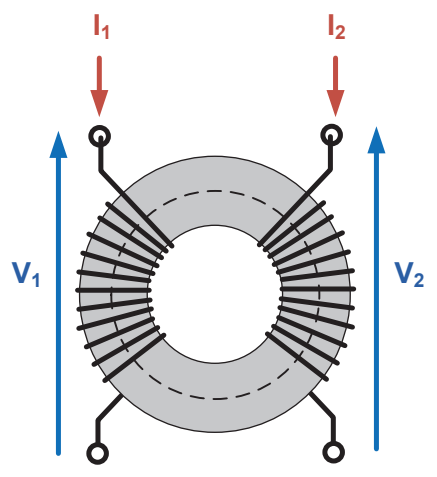

(a) 2-phase

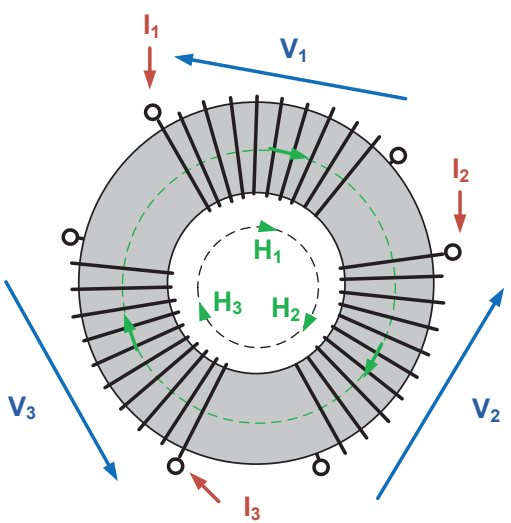

(b) 3-phase

Figure 4.12: Sketches of CMC.

\section{Dominant Capacitance}

The investigation has been performed for three sectionally wound CMCs (Figure $4.13 \mathrm{a}$ ) with increasing number of windings. They have been measured in two configurations shown in Figure 4.14. Their measured common mode impedance $Z_{c m}$ and phase impedance $Z_{\text {phase }}$ are shown in Figure 4.16 a. As expected, their inductance value increases with increased amount of turns. This is also confirmed by the physical derivation of the inductance value of toroidal 
inductor given by [79].

$$
L=\frac{\mu N^{2} A}{l}=\frac{\mu N^{2} h \ln \frac{d_{o}}{d_{i}}}{\pi} \text { (toroid) }
$$

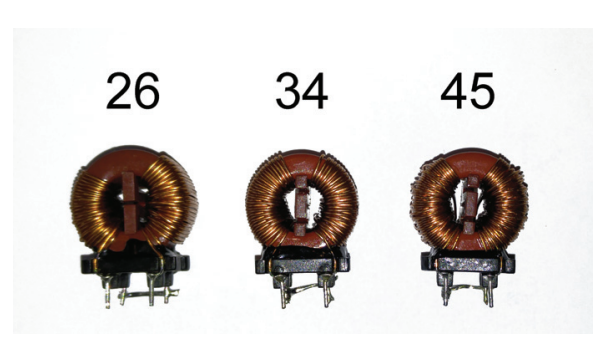

(a) Datasheet values are $5 \mathrm{mH}, 10 \mathrm{mH}$ and $20 \mathrm{mH}$ and their number of turns from left to right. The core material is MnZn.

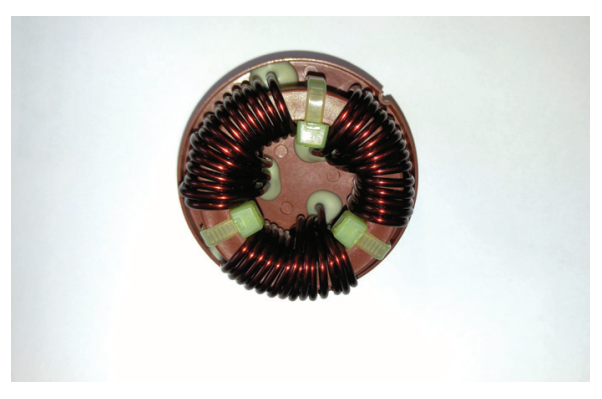

(b) Data-sheet value is $3.5 \mathrm{mH}$ with a Vitroperm noncrystalline core.

Figure 4.13: The measured chokes are from left to right: WE-CMB744823-305, WE-CMB744823-210, WE-CMB744823-220, and VAC T60405-S6123-X225.

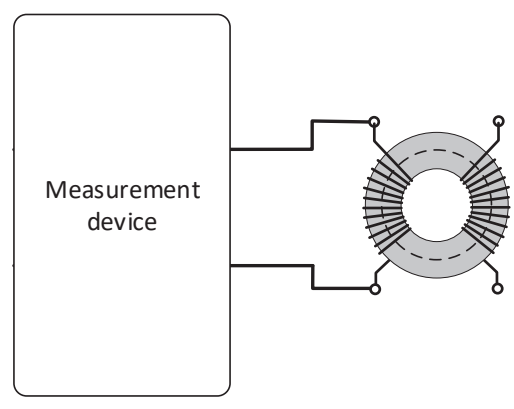

(a) Phase impedance, also known as line impedance

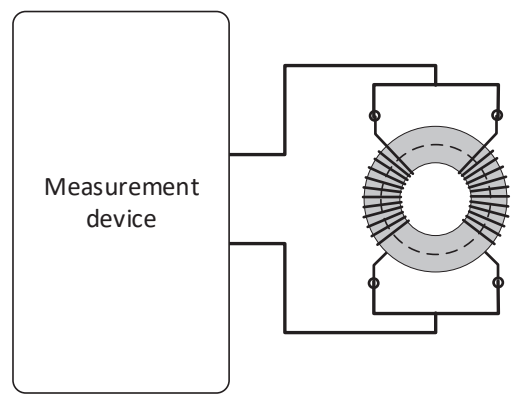

(b) Common Mode impedance

Figure 4.14: Impedance measurement, adopted from [71].

Figure 4.16a and Table 4.2 show that the EPC of the choke with $N=45$ is lower than in the case of $N=34$. Which is contradictory to the assumption: "the amount of turns is directly related to the total $E P C$ ". To investigate the capacitive behavior and compare the different chokes, the measured ratios between CM-EPC and Phase-EPC are displayed in Figure 4.16b according to:

$$
\text { Ratio }=\frac{E P C_{c m}}{E P C_{\text {phase }}}
$$




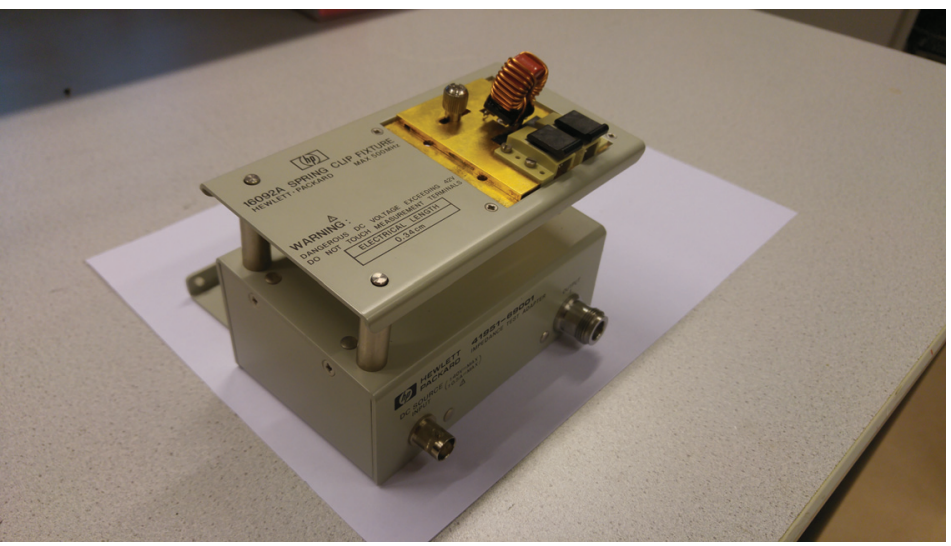

Figure 4.15: Test fixture used from the impedance measurements as shown in Figure 4.14. It is the HP16092A spring clip fixture that can accurately be used in conjunctions with the Keysight E5061B to approximately $500 \mathrm{MHz}$ after de-embedding.

In the previous section it was already mentioned, that a ratio of 2 is expected. As a CMC consists of two inductively coupled inductors in parallel. In the high frequency domain the inductors act as capacitors, and therefore should add up in the parallel case.

In Figure $4.16 \mathrm{~b}$, the resonant peak of the $Z_{c m c}$ curve can be seen as the first asymptote. In each of the cases, the EPC ratio is equal to 1, up to a certain higher frequency. Even though the assumption of more turns is higher capacitance doesn't hold, the ratio between phase and CMC EPC is constant within a certain bandwidth. As the parasitic capacitance exhibits a more complex behavior than a mere physical capacitance would suggest, further investigation is required. In the next section mutual coupling through the core-material is investigated.

Table 4.2: Measured values for the three investigated chokes with amount of turns $\mathrm{N}$.

\begin{tabular}{|l||c|c|c|c|}
\hline Chokes & $\begin{array}{l}\mathrm{L}[\mathrm{mH}] \\
@ 100 \mathrm{kHz}\end{array}$ & $\begin{array}{l}\mathrm{EPC}[\mathrm{pF}] \\
\text { @1 MHz }\end{array}$ & EPR $[\mathrm{k} \Omega]$ & Ratio EPC \\
\hline $\mathrm{N}=26$ & 5.2 & 17.7 & 28 & 1 \\
\hline $\mathrm{N}=34$ & 11.9 & 47.4 & 68 & 1 \\
\hline $\mathrm{N}=45$ & 27.2 & 42.2 & 144 & 1 \\
\hline
\end{tabular}




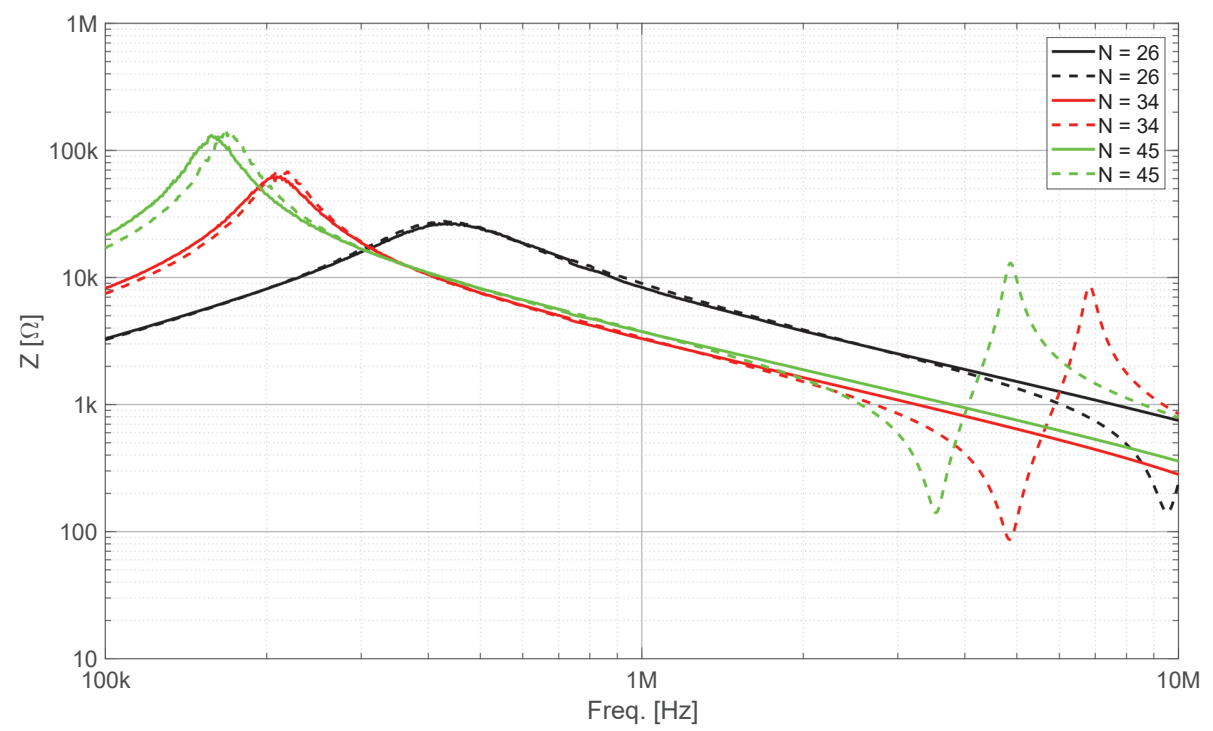

(a) Common mode $Z_{c m}$ and phase impedance $Z_{\text {phase }}$, solid and dashed displayed respectively.

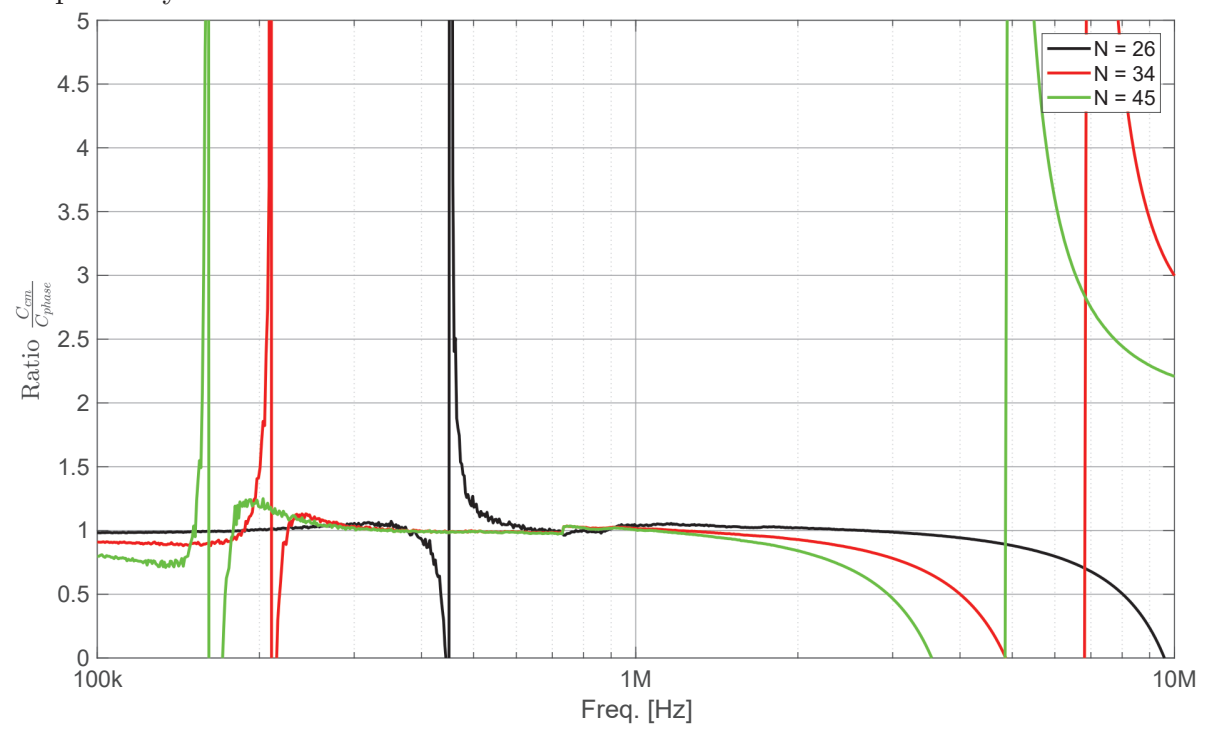

(b) Measurement of EPC with impedance analyzer

Figure 4.16: Impedance measurement. 


\subsubsection{Mutual Coupling Effect}

A relative simple model of a CMC consists of two parallel placed and inductively coupled inductors. In section 4.2.1 it has been shown by using Equations 4.11 to 4.14, to calculate the values of the equivalent circuits displayed in Figure 4.1, one is able to model a CMCs impedance. However, based on the assumption that the total parasitic capacitance increases by placing two identical inductors in parallel, the high frequency behavior of the CMC should not be equal to that of a single inductor.

In this subsection two identical three-phase CMCs were used to investigate the mutual coupling between phases and its effect on the impedances. The measurements have been done for combinations between 2 , and between 3 windings. For simplicity, only the measurements of 2 phases are shown.

In case of common mode currents the (non-ideal) inductors are connected parallel wise as shown in Figure 4.10c. They have near perfect inductive coupling for low-frequencies. In this case, we define low-frequencies as those that are below the first resonant frequency of the choke. The ferromagnetic or nano-crystaline core provides an inductive coupling that influences a choke's behavior differently for DM and CM currents. The mutual induction between two inductances has been defined with Equation 4.14.

Assuming near perfect coupling $(k \approx 1)$ and identical winding $L_{1} \approx L_{2}=$ $L$, one can calculate the resulting impedance. A CM measured CMC (Figure $4.14 \mathrm{~b}$ ) would have an inductance equal to $L$.

$$
\frac{\left(L_{1}+M\right) \cdot\left(L_{2}+M\right)}{\left(L_{1}+M\right)+\left(L_{2}+M\right)}=\frac{(L+M)}{2}=L
$$

while DM measured (Figure 4.17d) it would become approximately zero:

$$
\frac{\left(L_{1}-M\right) \cdot\left(L_{2}-M\right)}{\left(L_{1}-M\right)+\left(L_{2}-M\right)}=\frac{(L-M)}{2} \approx 0
$$

A VNA with impedance analyses feature has been used to measure the chokes in multiple configurations. As explained only two out of three phases are shown, however the measurements have also been performed with a third identical choke. This resulted in similar results, however scaled to the amount of used phases. The ratios have again been calculated with respect to a single phase measurement (Figure 4.14a). The following configurations were used in the measurement setup:

1. series-decoupled (Figure 4.17a)

2. parallel-decoupled (Figure 4.17b)

3. series-coupled (Figure 4.17c)

4. parallel-coupled (Figure 4.14b) 
Which considers the phases as single inductors, that can be connected in series or in parallel. Either on the same core (coupled, $M=L$ ) or on different cores (decoupled, $M=0$ ). Note however that in case of the coupled series connection the flux of the phases are additive in nature, as opposed to a DM measured CMC. As the coupling is primarily inductive in nature, it is expected that it has no influence on the EPC. Based on these assumptions Table 4.3 summarizes the expected values for each configuration. The DM results have been omitted in the graphs and table to reduce display complexity.

Table 4.3: Expected values for two phases and their approximated measured values from Figure 4.18 .

\begin{tabular}{|l||c|c||c|c|}
\hline & Exp. L & Meas. L & Exp. EPC & Meas. EPC \\
\hline $\begin{array}{l}\text { ser.-decoup. } \\
\text { (Fig. 4.17a) }\end{array}$ & $L+L=2 L$ & $\approx 2 L$ & $\frac{1}{\frac{1}{C}+\frac{1}{C}}=0.5 C$ & $\approx 0.5 C$ \\
\hline $\begin{array}{l}\text { par.-decoup. } \\
\text { (Fig. 4.17b) }\end{array}$ & $\frac{L \cdot L}{L+L}=0.5 L$ & $\approx 0.5 L$ & $C+C=2 C$ & $\approx 2.3 C$ \\
\hline $\begin{array}{l}\text { ser.-coup. } \\
\text { (Fig. 4.17c) }\end{array}$ & $2(L+M)=4 L$ & $\approx 4.1 L$ & $\frac{1}{\frac{1}{C}+\frac{1}{C}}=0.5 C$ & $\approx 0.5 C$ \\
\hline $\begin{array}{l}\text { par.-coup. } \\
\text { (Fig. 4.14b) }\end{array}$ & $\frac{(L+M)^{2}}{2(L+M)}=L$ & $\approx L$ & $C+C=2 C$ & $\approx C$ \\
\hline
\end{tabular}

By comparing the results from Figure 4.18, which are summarized in Table 4.3, one can see the expected inductances agree. In contrast to the expected EPC values. In case of two decoupled parallel phases the equivalent parasitic capacitance is additive and the ratio becomes $\approx 2$, while in the coupled case the ratio becomes 1 again. It appears the EPC is influenced by an inductive coupling. Therefore we can conclude the simplistic model as described in section 4.2.1 holds for low frequencies only. Also the assumption that the capacitive behavior of a CMC is merely a sum of physical capacitances seem to be a common misconception, this however has to be investigated. Further research is required to produce a high frequency model of a CMC that incorporates physical inductive effects at these frequencies. Hence this chapter continues with the development of a full wave CMC simulation model.

\subsection{CMC - 3D Full Wave High Frequency Modeling}

The results presented in the following section are published in IEEE Transaction on Electromagnetic Compatibility [55].

It has been shown in [80] that mutual coupling between components can be investigated using 3D simulation software. FEKO, CST and Ansys all provide the possibility for complex Electro-Magnetic (EM)-model simulations, however circuit designers often do not understand, and thus lack training in, EM fields. 


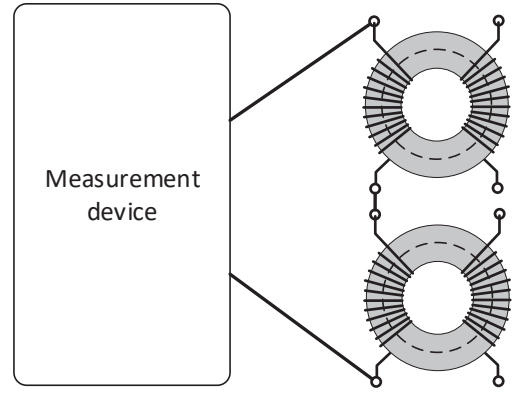

(a) Decoupled series impedance

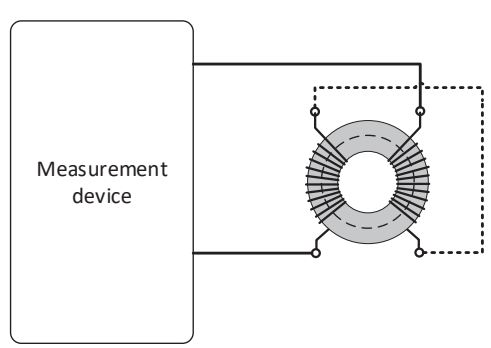

(c) Coupled (constructive) series (d) DM i.e. Coupled (deconstructive) seimpedance

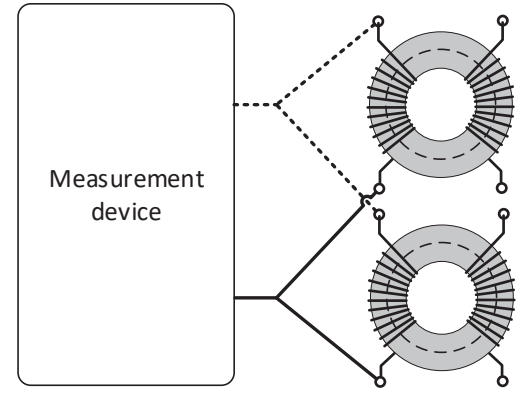

(b) Decoupled parallel impedance

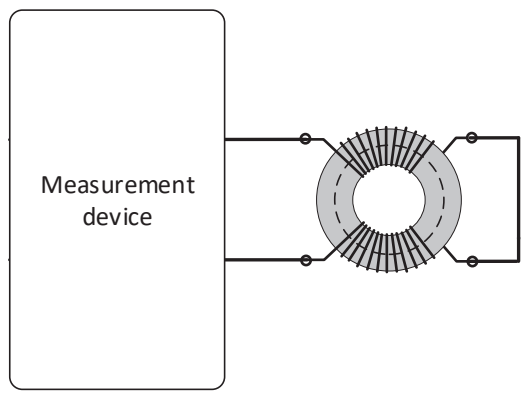
ries impedance

Figure 4.17: Impedance measurement, adopted and extended from [71]. 


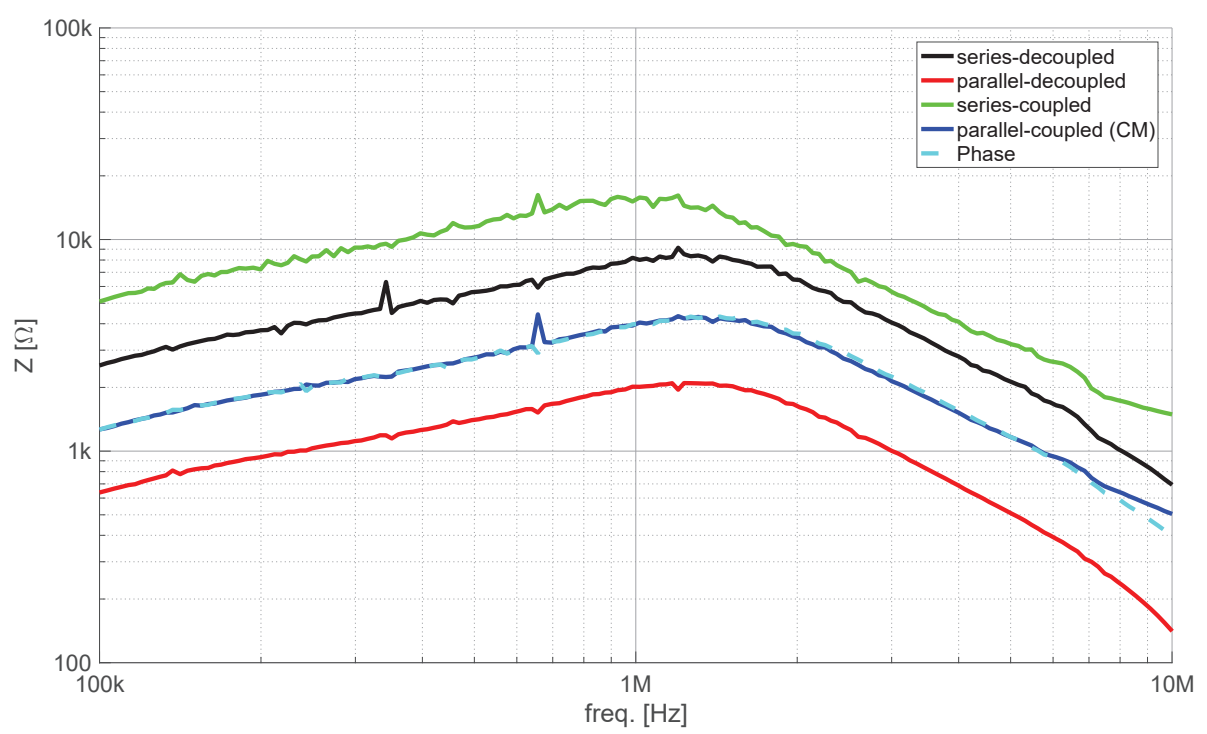

(a) Impedance

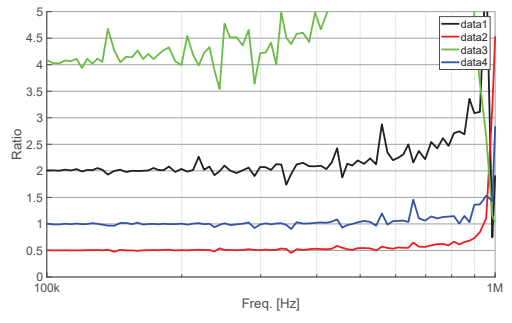

(b) Inductance ratios

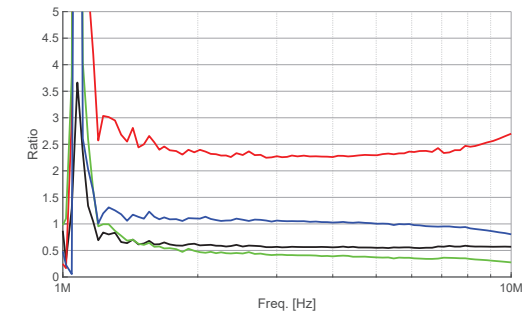

(c) Capacitance ratios

Figure 4.18: Impedance measurements with the extracted inductance and capacitance values. 
The modeling procedure developed is aimed at providing a hands-on guide for 3D CMC modeling, while it eventually can contribute to automated field effect extraction and back annotation into circuit simulators. Challenges that are addressed in this section are the geometrical modeling, incorporating frequency dependent complex permeability and eventually extracting the impedances of the choke that are needed by the designer, together with an investigation on dependencies of parameters.

The 3D model that is described in section 4.3.1, can be used to investigate effects that are often assumed to be dominant or hard to control. It also allows for prediction of in-situ parasitic effects. These have a significant impact in performance of the final product. It is followed by an S-parameter based impedance extraction method in section 4.3.2. In a "2-phase" CMC case, the simulation has four single ended ports representing a four port VNA setup. Then the results are presented in section 4.3.3, first verifying the simulation and later identifying the most critical parts of the component and measurement setup that need to be accurately modeled. This was done not only for the DM and CM impedance, but also for leakage inductance, inter-winding impedance, and phase impedance. Their relation to parasitic effects are elaborately described in [71]. The results shown are for a typical CMC available in the EMI filter design kit from Würth Elektronik, and a ferrite core from FerroxCube that is wound manually.

\subsubsection{Modeling 3D: FEM}

In this section a detailed description is given about modeling a toroidal shaped, sectional wound CMC. The main elements of the simulation procedure are discussed, as well as the challenges that arose during the modeling. This should be useful for anyone creating their own simulation setup or eventual parametric study. One can consider this to be used as a cookbook recipe for creating a 3D choke model opening a world of possible different recipes with some examples shown in Figure 4.19.

\section{Core}

The three main parameters used in the design of a toroidal core material are: inner radius $\left(r_{i}\right)$, outer radius $\left(r_{o}\right)$ and height $(h)$. A 3D-polygonal line is used to draw the center of the core material. From here, the full $360^{\circ}$ are covered by incrementing single degrees. To reduce meshing complexity, it is advised to increase the step size. From here, a cross section of the toroid is drawn and swept along the previous drawn circle. The models used here have a rectangular cross section. The 3D-model of the core is now described, and only the material parameters are missing. The inclusion of the exact complex material properties are crucial in correctly modeling a CMC in a 3D full wave simulator. 


\section{Ports}

In [81] and [76] it was shown that different techniques exist in measuring CMCs. Either with balanced or unbalanced methods in two or four port measurement setups. A four port model is used to investigate a multitude of parasitic effects with a single $3 \mathrm{D}$ simulation. The setup that has been investigated is shown in Figure 4.19a. It is good practice to avoid lengthy discrete ports, due to possible simulation errors resulting from either increasing parasitics or meshing issues [82]. The port length depends on the height above the ground plane, which is often much less than the separation distance between the leads of the CMC.

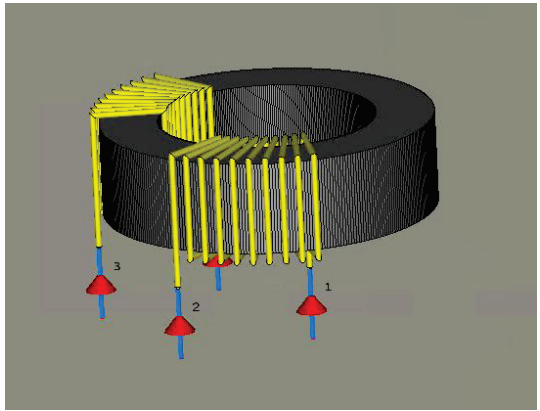

(a) FerroxCube TX22

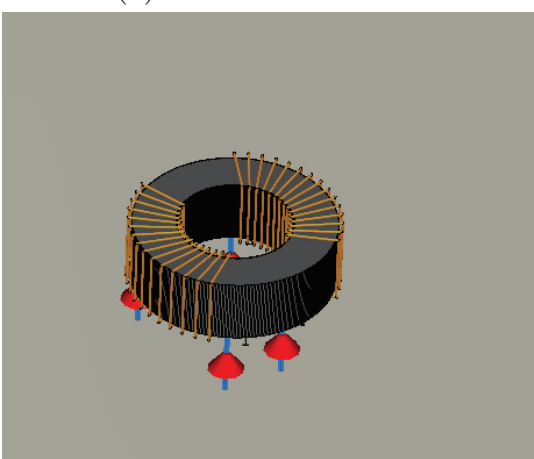

(c) Large winding angle

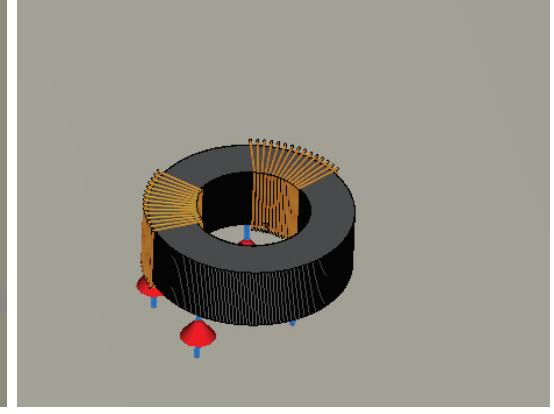

(b) Small winding angle

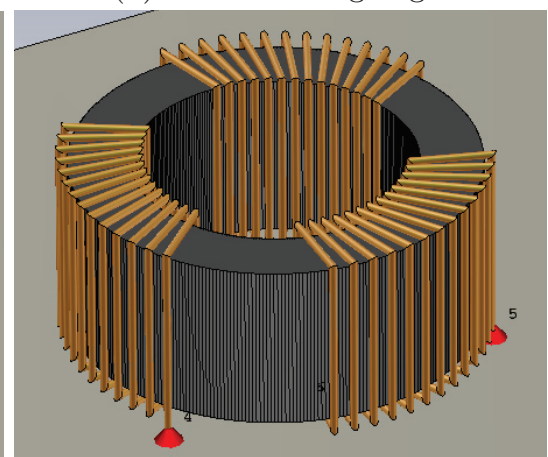

(d) 3-phase simulation option

Figure 4.19: Multi-port Simulations.

\section{Windings}

Windings are created with a polygonal curve, with each turn having four sets of $x, y, z$ parameters. $x$ and $y$ are calculated from a circular motion around the center of the core material.

$$
x=r \cdot \cos (\phi), \quad y=r \cdot \sin (\phi)
$$


The main limitation of this method of winding is its single layer and therefore limiting the number of turns and/or thickness of the wire.

By adding a parameter to account for layers of winding, the method could be extended to multi-layer winding. One has to be careful that the resulting structure is not overlapping anywhere, as it will be considered to be a single solid. i.e. if the turns are overlapping in $3 \mathrm{D}$ space, the wire acts as a foil that is wrapped around the core material.

\section{Boundary, Frequency range, Background material}

An important aspect of the 3D simulation environment are the boundary conditions in which the component is simulated. Important to notice is the amount of background material and type. They should represent the physical situation. In case of the measurements, the background material should be set to normal, which represents air, while the distance to the simulation boundaries should be sufficient (i.e. approx. a quarter of the maximum wavelength). Also the boundaries used are open. Note however, that one boundary will be set to Perfect Electric Conductor (PEC) to be electrically grounded. This represents the surface of the table at which the components were measured.

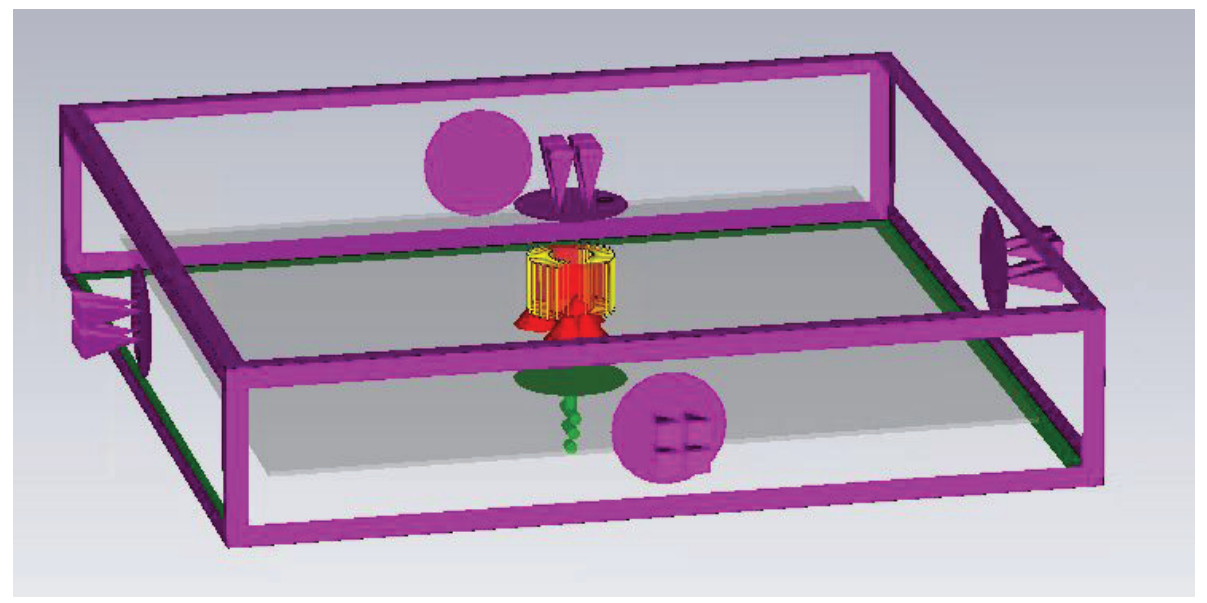

Figure 4.20: Boundary simulation setup.

\section{Solver: Frequency Domain}

As [13] mentioned, the solver was set to the frequency domain to incorporate the magnetic permeability dispersion model for materials at high frequencies. The 3D simulation will then be performed with a Finite Element Method (FEM) in CST Microwave studio. Defining the amount frequency points and their spacing will be directly related to simulation accuracy and duration. Good practice is to define the frequency points related to the used dispersion model. 
i.e. select points on curve bends and other parts of the permeability curves that are not linear interpolatable. As the most important parameter in designing the simulation, the magnetic permeability is elaborated on in the next subsection.

\section{Permeability}

Exact complex permeability values with a large frequency range are often difficult to acquire. Datasheet values have a challenge of often being limited at their frequency range and the accuracy during the data extraction method. Most of them also lack complex values. Permeability measurement techniques can be found in [83-86], each technique has their benefits and disadvantages. Two core materials are investigated in this section, the FerroxCube TX22/14/6.43E27 and the CMC WE-CMBM744823-601 (Figure 4.21). Note that based on production process and differences between datasheets of the same material deviations of $20 \%$ to $30 \%$ are possible and not uncommon. To increase the frequency range of the simulation model, the complex impedance of the inductors have been measured and the complex permeability is extracted as described in $[86]$.

$$
\mu^{\prime}-j \mu^{\prime \prime}=\frac{-j\left[\mathcal{Z}_{r}+j \mathcal{Z}_{i}\right]}{\mu_{0} f N^{2} h \ln \frac{d_{o}}{d_{i}}} \text { (toroid) }
$$

It is not in the scope of this thesis to address the accuracy as we focus on the modeling parameters of the inductors, like core width and height. A short overview of possible permeability extraction methods can be seen in Appendix E.

\section{Permittivity}

Next to permeability, the core material also has a dielectric permittivity. The effect on the CMC parasitic behavior can be investigated in a similar manner as done for permeability, by including a frequency dependent permittivity curve in the simulation material, however the investigation is simplified in this thesis by including a constant permittivity. This allows for a sensitivity analysis to determine the impact of the parameter on the resulting impedances.

\section{Simulation Output}

FEM simulation programs can have an infinite amount of different output files. They can contain information about voltages at different points in the simulation space, or current density in a certain cross-section of the 3D structure, and even electric and magnetic field information. From this point on, we assume that the software is capable of providing complex valued S-parameters for each of the simulation ports. In the setup depicted in Figure 4.19a this would result in a $4 \times 4 \times n$ matrix, with $n$ being the number of simulated frequency points. 

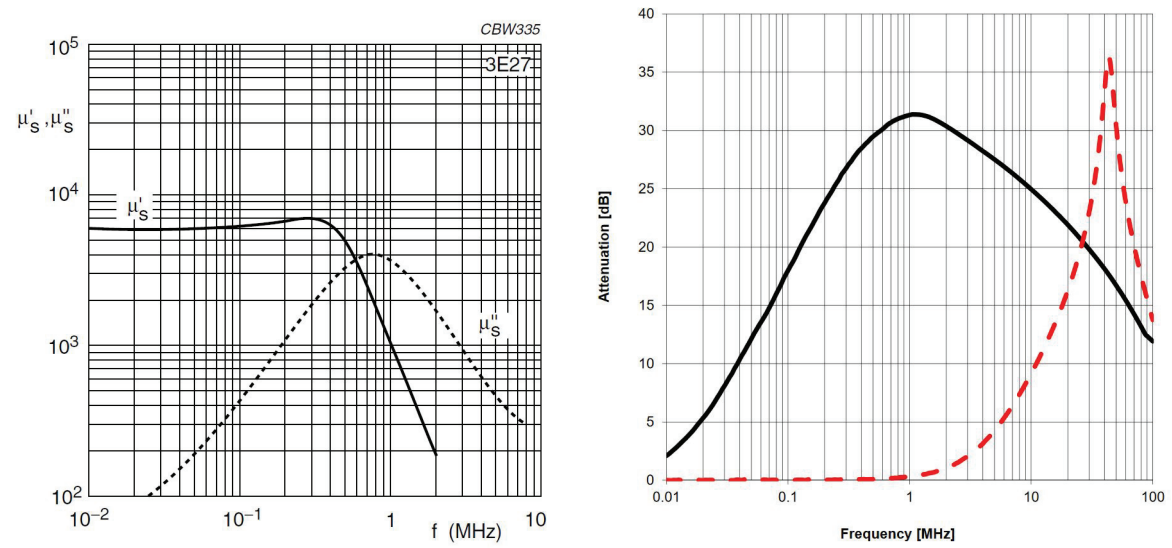

(a) Permeability as shown in the (b) Attenuation as shown in the datasheet datasheet for Ferroxcube 3E27 material of WE-CMBM744823-601

Figure 4.21: Datasheet examples.

\subsubsection{Impedances Extraction}

Extracting the DM and CM impedance of a simulated (or measured) common mode choke from S-parameters, requires a set of transformations based on the measurement or simulation method. A derivation is given for the 4-port (single ended) setup is given, based on the work in [81]. Note that the order of the ports and thus the location of the ports are crucial when using the manipulation matrix $\mathbf{M}$.

\section{S-parameter mode decoupling}

In case of using four ports, one can convert the single ended 4-port measurement results into mixed mode S-parameter set, from which a CM and DM impedance can be derived. Notice that the order of the ports has to be taken correctly, depending on the simulation.

$$
\begin{gathered}
\mathbf{S}=\left[\begin{array}{llll}
S_{11} & S_{12} & S_{13} & S_{14} \\
S_{21} & S_{22} & S_{23} & S_{24} \\
S_{31} & S_{32} & S_{33} & S_{34} \\
S_{41} & S_{42} & S_{43} & S_{44}
\end{array}\right] \\
\mathbf{S}_{m m}=[\mathbf{M}] \mathbf{S}\left[\mathbf{M}^{-1}\right]
\end{gathered}
$$

with M:

$$
\mathbf{M}=\frac{1}{\sqrt{2}}\left[\begin{array}{cccc}
1 & -1 & 0 & 0 \\
1 & 1 & 0 & 0 \\
0 & 0 & 1 & -1 \\
0 & 0 & 1 & 1
\end{array}\right]
$$


Resulting in:

$$
\begin{gathered}
\mathbf{S}_{\mathbf{m m}}=\left[\begin{array}{ll}
\mathbf{S}_{m m, 11} & \mathbf{S}_{m m, 12} \\
\mathbf{S}_{m m, 21} & \mathbf{S}_{m m, 22}
\end{array}\right] \\
\mathbf{S}_{m m, i j}=\left[\begin{array}{ll}
\mathbf{S}_{d d, i j} & \mathbf{S}_{d c, i j} \\
\mathbf{S}_{c d, i j} & \mathbf{S}_{c c, i j}
\end{array}\right] \\
Z=Z_{0} \frac{\left(1+S_{11}\right)\left(1+S_{22}\right)-S_{21} S_{12}}{2 S_{21}}
\end{gathered}
$$

Note that the resulting matrix of eq. 4.20 is a 4 by 4 matrix as it consists of four mixed mode matrices. Selecting the differential mode parts $\left(S_{d d, i j}\right)$, will result in a 4 element matrix that can be applied to Equation 4.22 and will result in DM impedance of the choke. The same holds for $S_{c c, i j}$, resulting in the CM impedance.

\section{Additional Impedances}

Following the 4-port simulation approach, it is now possible to investigate a CMC's designable parameters influence on $Z_{c m}$ and $Z_{d m}$ under the assumption the incorporated permeability is correct. However, from [71] it can be seen that $Z_{c m}$ and $Z_{d m}$ are not enough to accurately derive a behavioral model. An interesting feature of 4-port simulation (and measurements) of CMCs is the possibility to also investigate the phase impedance $\left(Z_{p h}\right)$, inter-winding impedance $\left(Z_{\text {int }}\right)$ and leakage inductance simultaneously by mathematically introducing the measurement setups as presented in Figure 4.22.
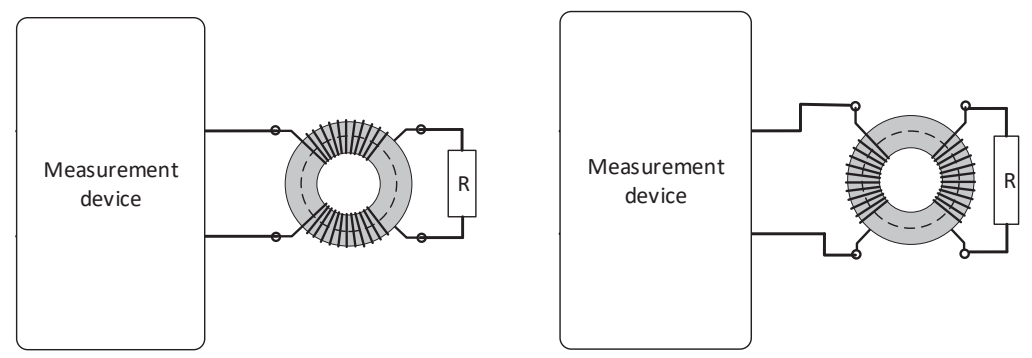

(a) Transistion between interwinding (b) Transitions between leakage induccapacitance and DM impedance tance to phase impedance

Figure 4.22: Physical representation of mathematically applied resistors. $R$ is increasing from approx. $1 \Omega$ to $10 \mathrm{M} \Omega$, this mimics the short to open circuit measurements. 


\subsubsection{Results}

The previous sections have shown how to model a CMC and how to extract the multiple useful impedances from the S-parameter simulation. The methods are now applied to determine the dependency on designable parameters. In table 4.4 an overview of the parameters investigated is shown. It shows qualitatively how important a parameter is to be modeled perfectly. It summarizes the sensitivity of the resulting impedances, which is displayed in the Figures on the right hand side. The sensitivity as is defined in [87] is computed using:

$$
\text { Sensitivity }=\kappa=\frac{\sigma_{\text {out }} / \mu_{\text {out }}}{\sigma_{\text {in }} / \mu_{\text {in }}}
$$

With $\sigma$ the standard deviation and $\mu$ the average of the output impedances and input parameters per frequency point respectively. The shown values have been limited from 0 to 2 . The lower the value, the less sensitive the output is for variations in input parameter. A value $\kappa=1$ represents a linear relation, while a value of $\kappa=2$ shows a quadratic relation. For summarizing the effect a parameter has on a certain impedance, we define 4 qualitative regions of sensitivity:

- none: $\kappa \approx 0$

- low: $0<\kappa<0.5$

- medium: $0.5<\kappa<1.5$

- high: $1.5<\kappa$

This can be applied to any design parameter defined in the simulation. A similar study performed with measurements can take hours or even days, while by automation one is able to do it within minutes.

First the simulation is verified for an inductor that is wound on the toroidal core measured in Figure 4.23. Then the results are presented based on the simulation setups as described in Figure 4.22, this verifies that 5 types of impedances can be extracted from a single 4-port simulation which are related to different designable parameters [88]. After the verification results, the investigation continues by presenting the results that show the effect of 6 parameters on the extracted impedances: Winding angle, height of the core, width of the core, height of the core above the ground plane, lead length, and wire radius. 
Table 4.4: Effect of 3D model parameters on simulated impedances for: $f \leq$ $100 \mathrm{MHz} / f>100 \mathrm{MHz}$.

\begin{tabular}{|l||l|c|c|c|c|}
\hline & Parameter & $Z_{C M}$ & & $Z_{D M}$ & \\
\hline Core & $\bullet$ Width & None/High & Fig. 4.26c & Low/High & Fig. 4.28c \\
& $\bullet$ Height & Med./Med. & Fig. 4.26b & Med./Med. & Fig. 4.28b \\
& $\bullet \mu$ & Highest & - & - & - \\
& $\bullet \epsilon$ & None/Low & Fig. 4.30a & None/Low & Fig. 4.30b \\
\hline Wire & $\bullet$ Radius & Low/Low & Fig. 4.26f & Low/Low & Fig. 4.28f \\
& $\bullet$ Turns & High $\left(N^{2}\right)$ & Fig. 4.24 & - & - \\
& $\bullet$ Angle & None/Med. & Fig. 4.26a & Med./Med. & Fig. 4.28a \\
\hline Setup & $\bullet$ Ht. abv. GND & None/Low & Fig. 4.26d & None/Low & Fig. 4.28d \\
& $\bullet$ Lead Length & None/None & Fig. 4.26e & None/Low & Fig. 4.28e \\
\hline Sim. & $\bullet$ Port Length & None/Low & Fig. 4.26d & None/Low & Fig. 4.28d \\
\hline
\end{tabular}

\begin{tabular}{|c|c|c|c|c|c|}
\hline & Parameter & $Z_{\text {int }}$ & & $Z_{p h}$ & \\
\hline \multirow[t]{3}{*}{ Core } & - Width & Low/Me & Fig. $4.29 \mathrm{c}$ & None/High & Fig. $4.27 \mathrm{c}$ \\
\hline & - Height & Med./Med. & Fig. $4.29 b$ & Med./High & Fig. $4.27 \mathrm{~b}$ \\
\hline & & None/Low & Fig. $4.30 \mathrm{c}$ & None/Low & Fig. $4.30 \mathrm{~d}$ \\
\hline \multirow[t]{2}{*}{ Wire } & $\begin{array}{l}\text { - Radius } \\
\text { - Turns }\end{array}$ & Low/Med. & Fig. $4.29 \mathrm{f}$ & Low/Low & Fig. $4.27 \mathrm{f}$ \\
\hline & - Angle & Med./Med. & Fig. $4.29 \mathrm{a}$ & None/Med. & Fig. $4.27 \mathrm{a}$ \\
\hline \multirow[t]{2}{*}{ Setup } & - Ht. abv. GND & Low/Low & Fig. $4.29 \mathrm{~d}$ & None/Low & Fig. $4.27 \mathrm{~d}$ \\
\hline & - Lead Lc & Low/Low & Fig. $4.29 \mathrm{e}$ & None/Low & Fig. $4.27 \mathrm{e}$ \\
\hline Sim. & - Port Length & Low/Low & Fig. $4.29 \mathrm{~d}$ & None/Low & Fig. $4.27 \mathrm{~d}$ \\
\hline
\end{tabular}




\section{Results for the Inductor}

The simulation results presented in Figure 4.24 show a good agreement with the measurement results up until approx. $100 \mathrm{MHz}$. The permeability used in each of the simulations was extracted from a single measurement. So increasing the turns was the only parameter that changed between the simulations, as a result the length of 'wire' increases with increased number of turns.

As Equation 4.19 shows, the permeability depends heavily on $N^{2}$. Counting the 'exact' number of turns is therefore crucial for permeability derivation via the winding measurement. Using an adapter measurement circumvents the ambiguity. Note however in the 3D model, the same dependency holds and should therefore also be modeled perfectly.

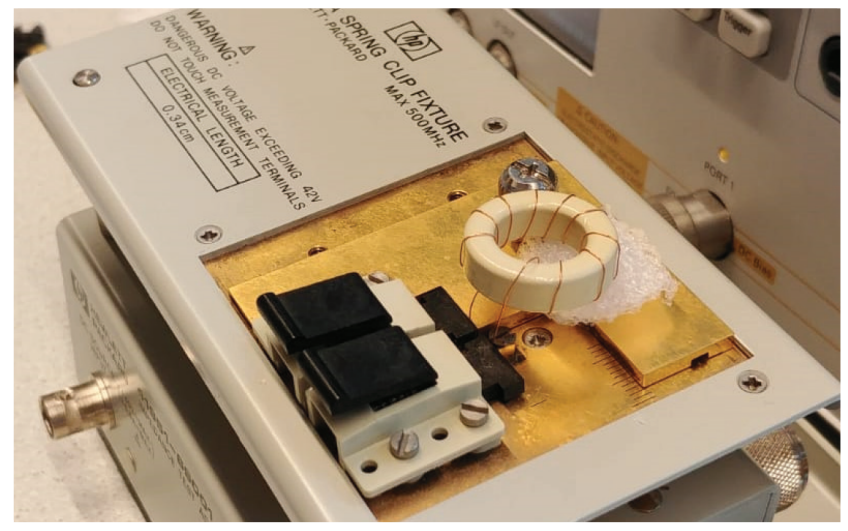

Figure 4.23: Winding measurement using the HP 16092A spring clip fixture.

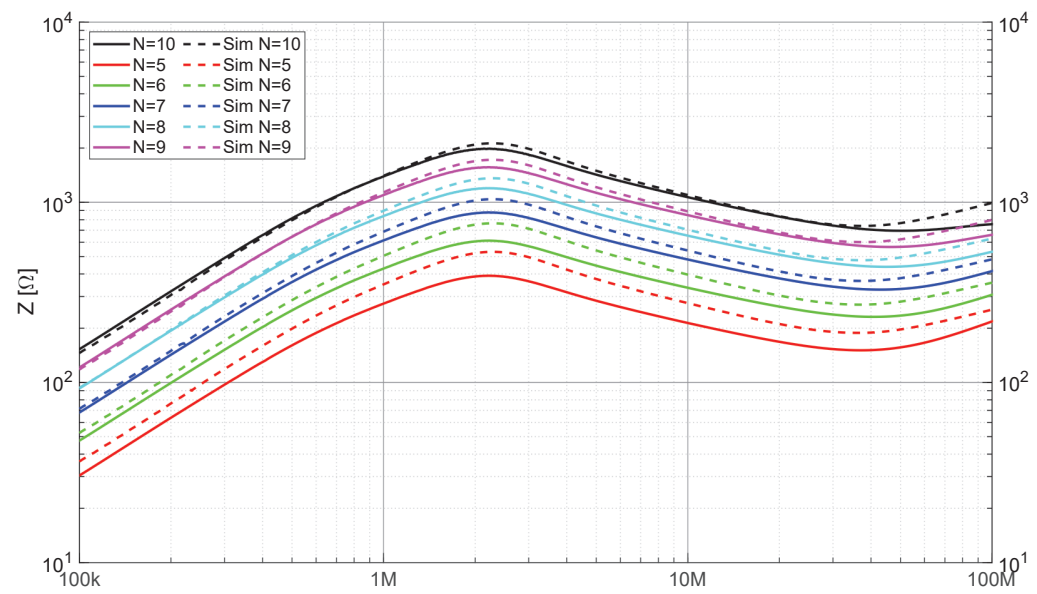

Figure 4.24: Measurement versus simulation of increased windings. 


\section{CMC Results}

Based on the 4-port S-parameters simulation results, the impedances in Figure 4.25 were derived. Note that it is derived from a single simulation result, and by mathematically terminating the choke as depicted in Figure 4.22 one can investigate the transition from a DM setup to an inter-winding capacitance setup (Figure 4.25a). This also holds for the phase impedance to leakage inductance setup, by mathematically terminating the other ports one can investigate this transition (Figure 4.25b).

The results presented in Figure 4.25 show the transition from one measurement to the other. This can have an interesting effect on how certain resonances are perceived and eventually modeled. But furthermore shows the possibility to derive 5 impedances from a single full wave simulation. The verification can be seen in the results presented in figures 4.26 through 4.29. Here next to the simulation derived results, the physical measurement results are presented together with a sensitivity curve. The effect a certain deviation of an input parameter has on the output parameter, i.e. different impedances, is summarized in Tab. 4.4. It is split into a low and high frequency region, $f \leq 100 \mathrm{MHz}$ and $f>100 \mathrm{MHz}$ respectively. The sensitivity shown in Figures 4.26 through 4.29 are related to the effect parameters have on the CMC model as described in [88]. The effect seen is also an indication on how important it is to perfectly model this part of the simulation. i.e. how much effort should be put in measuring these parameters of the physical object. As the effects can be easily seen in the figures, we only discuss the deviations and possible errors seen.

In Figure 4.27 it can be seen that the extracted phase impedance simulation results show a resonance at approximately $65 \mathrm{MHz}$, which is absent in the Figure 4.26. This also holds for the measurements performed, which can be seen as a dashed line in the figures. As the permeability is derived from CM impedance measurement performed on the choke, it is safe to say the resonance is not a material property but a geometric property. In case of the $\mathrm{CM}$ it appears the resonance is canceled. Figure 4.30 shows the influence of core's permittivity for the four extracted impedances. Relative permittivity values up to 27 have been chosen to be displayed, as these cover a wide variety of core materials. Note however relative permittivity up to 1000 has been simulated and the sensitivity calculated, which did not significantly alter the results due to low sensitivity. As expected, the low-frequency region is hardly sensitive to the permittivity, except for the interwinding impedance which is capacitive in nature and has a nearly constant sensitivity to the variation. 


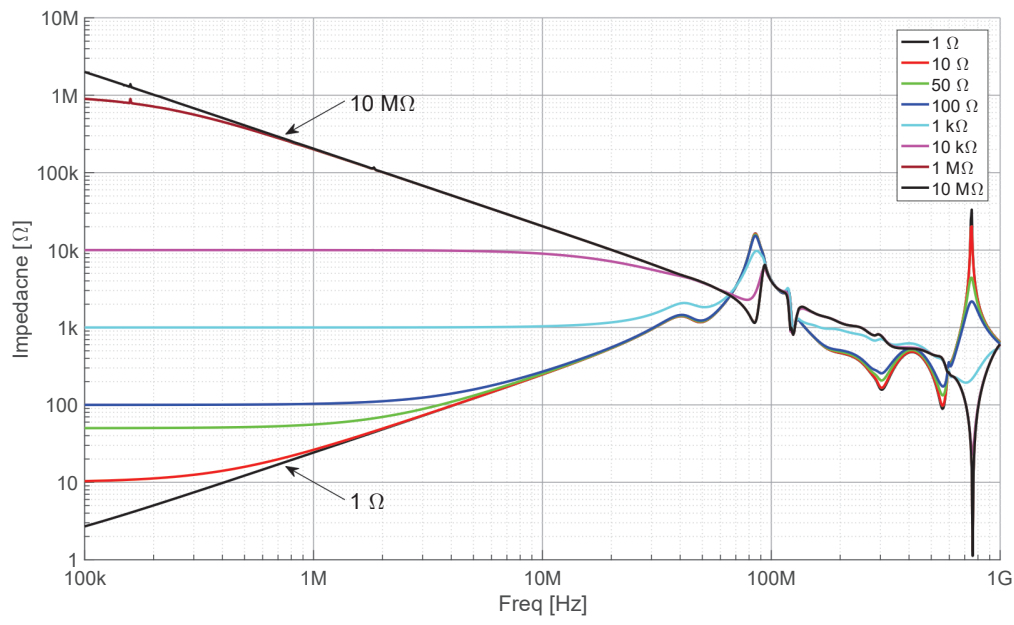

(a) DM to inter-winding impedance

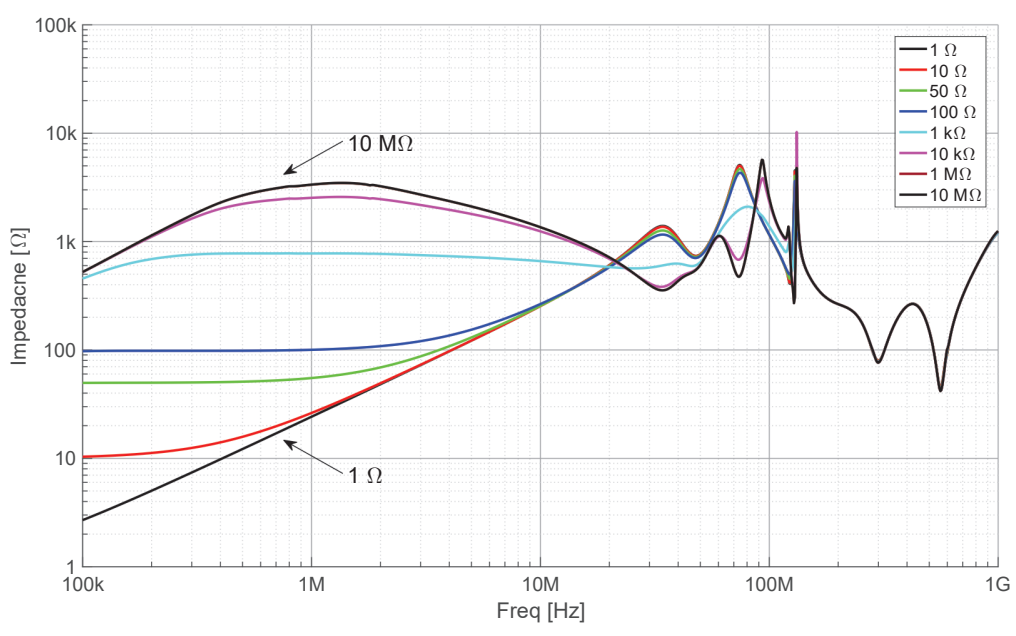

(b) Phase to leakage inductance

Figure 4.25: 4-port simulation results to impedances measurements by mathematical transformations as seen in Figure 4.22. 


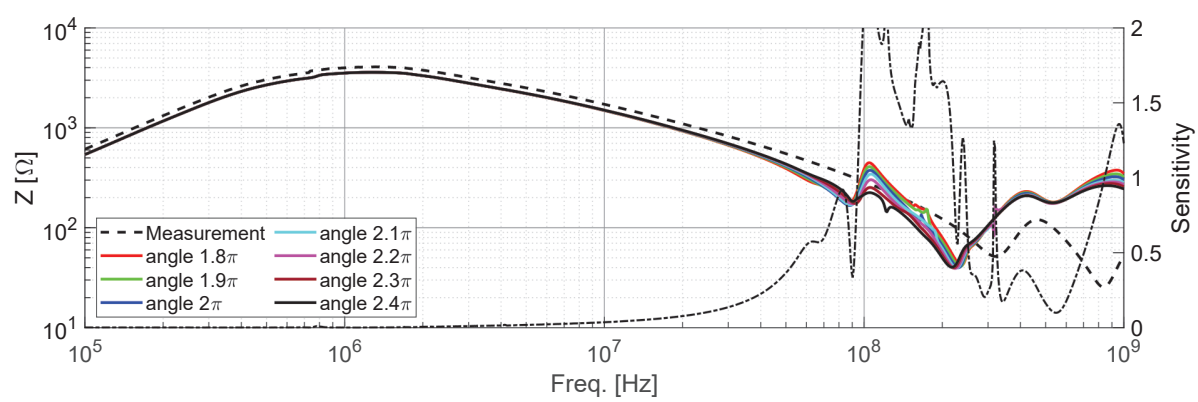

(a) Angle

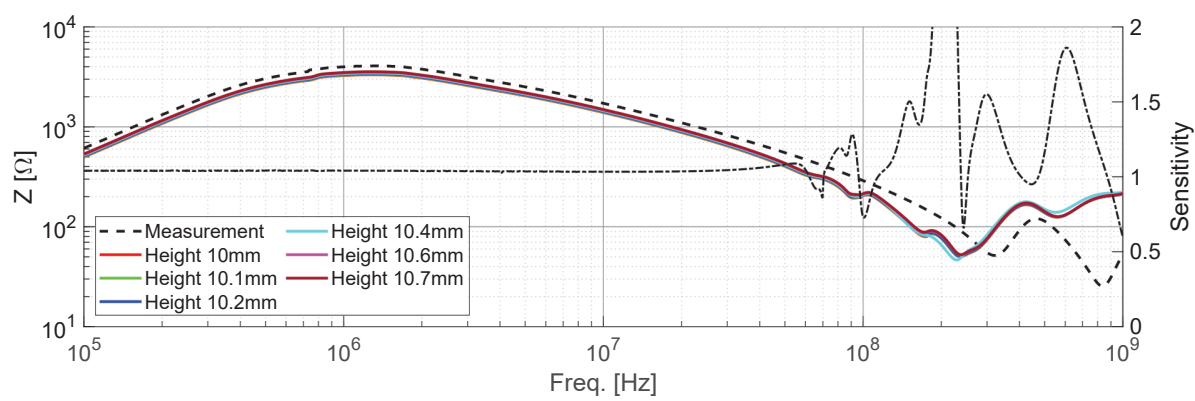

(b) Height

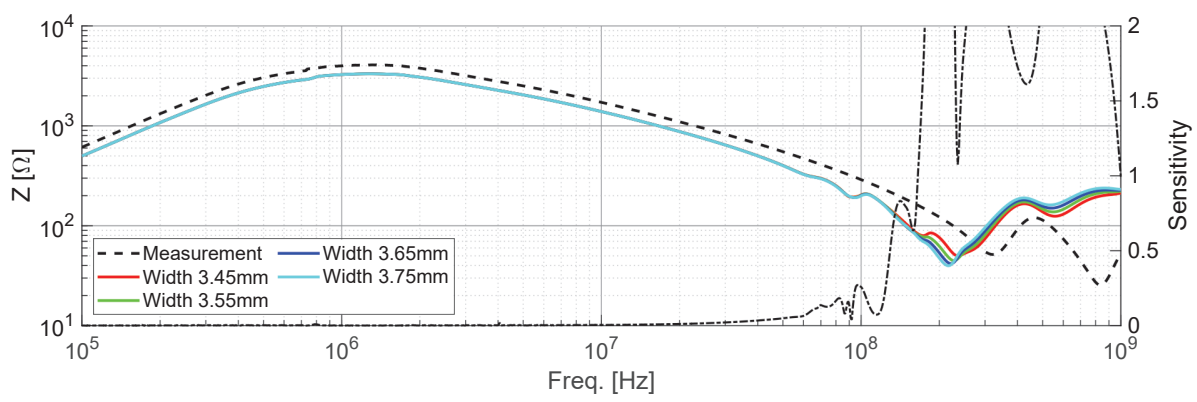

(c) Core width

Figure 4.26: CM impedance. 


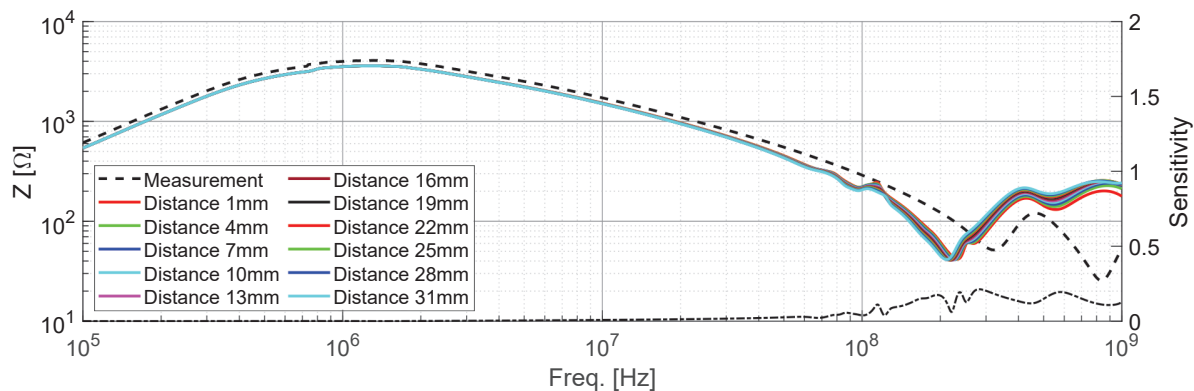

(d) Height above GND

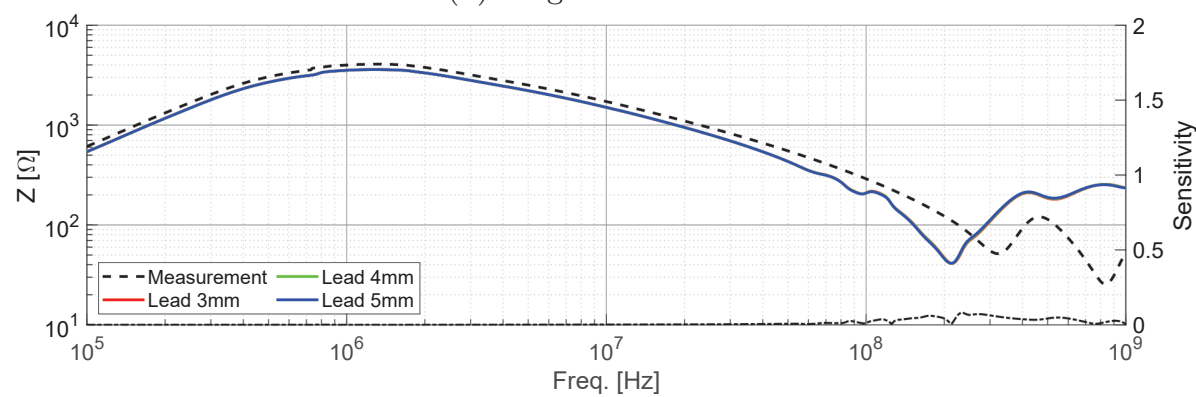

(e) Lead length

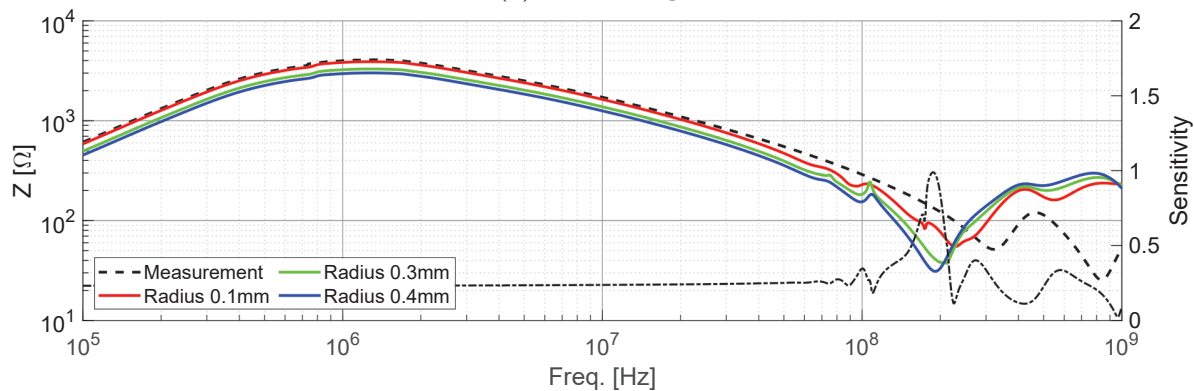

(f) Wire radius

Figure 4.26: CM impedance. 


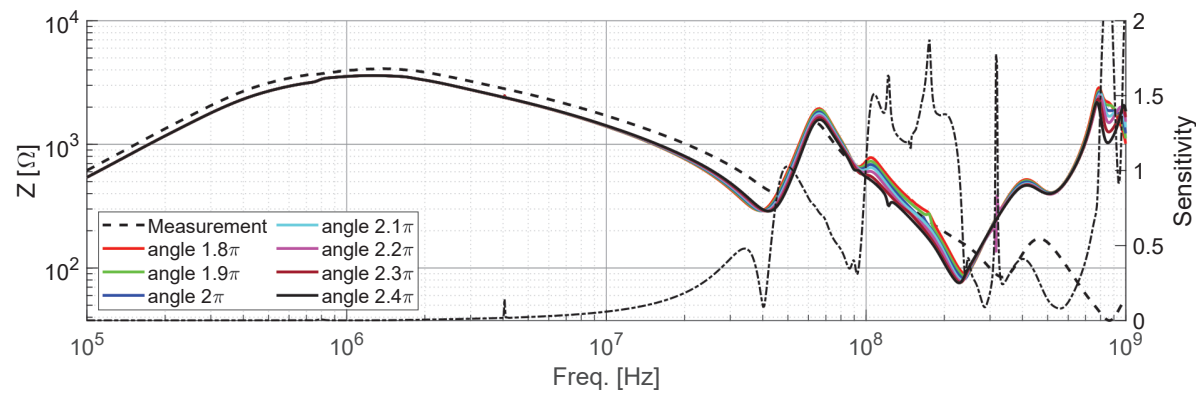

(a) Angle

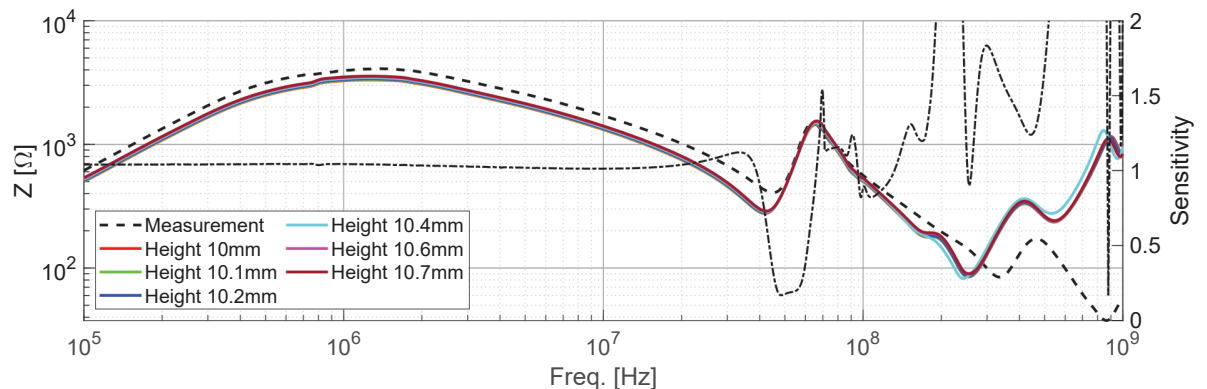

(b) Height

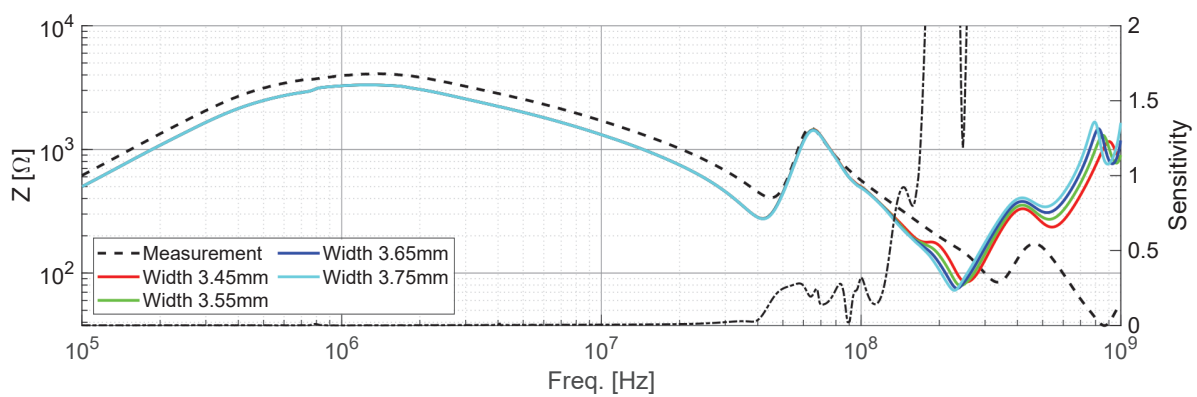

(c) Core width

Figure 4.27: Phase impedance. 


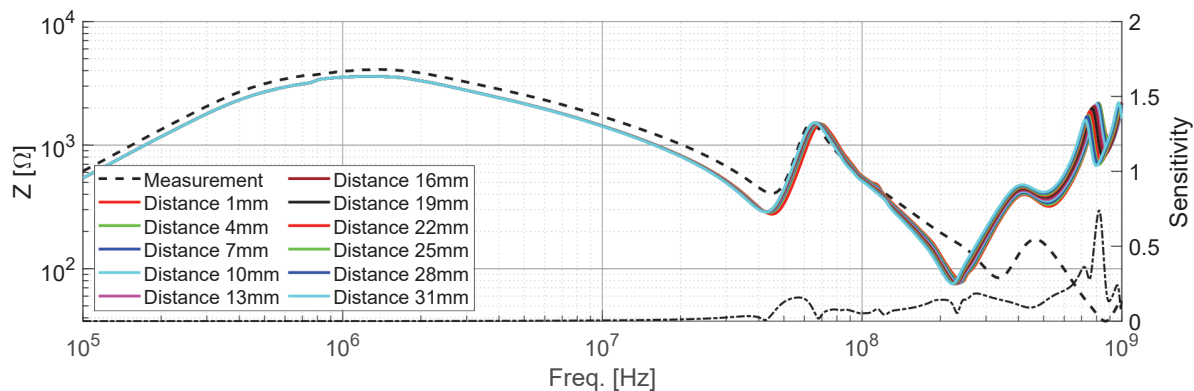

(d) Height above GND

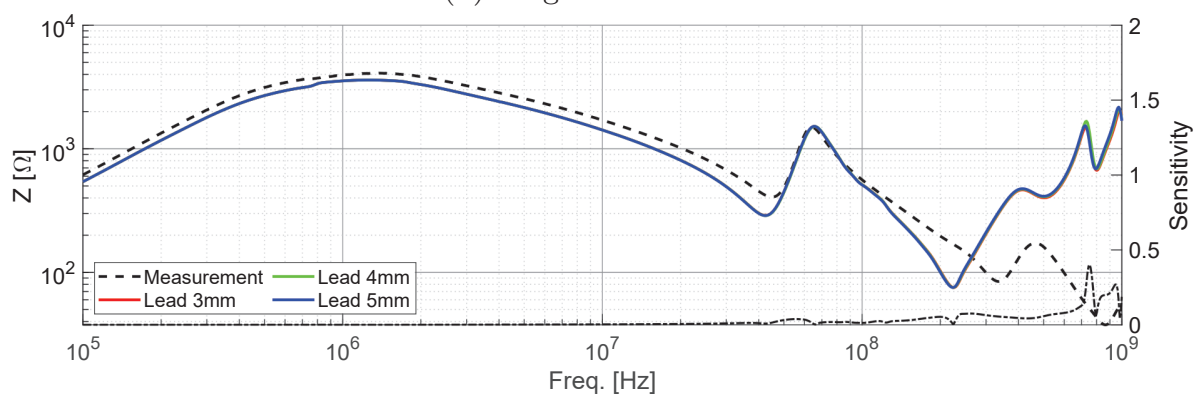

(e) Lead length

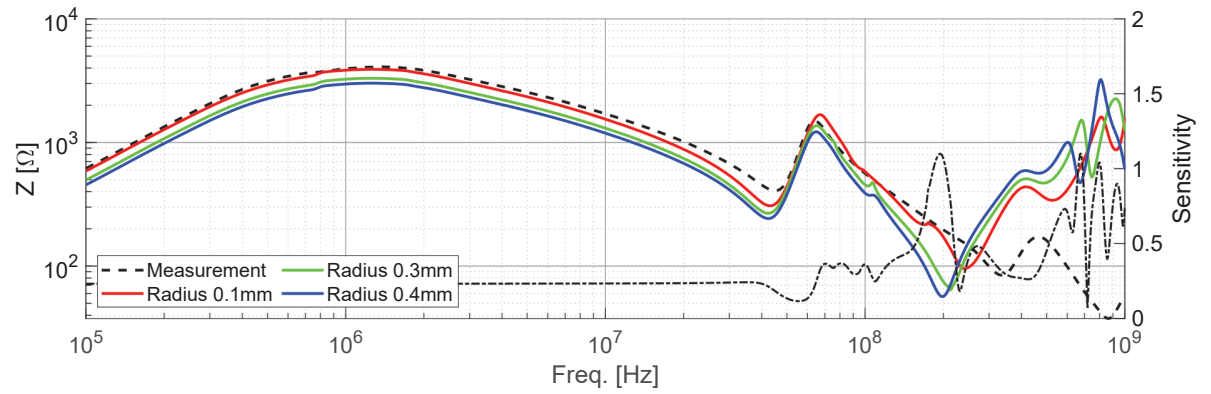

(f) Wire radius

Figure 4.27: Phase impedance. 


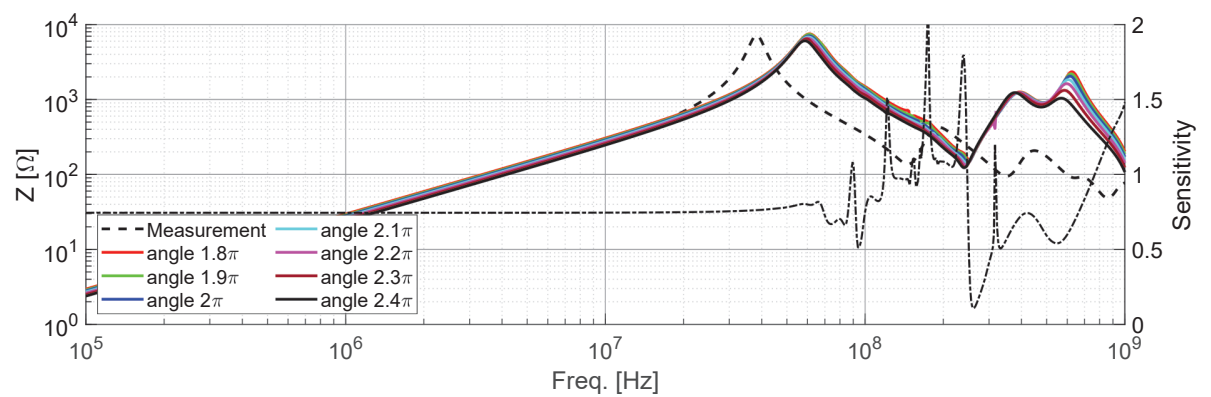

(a) Angle

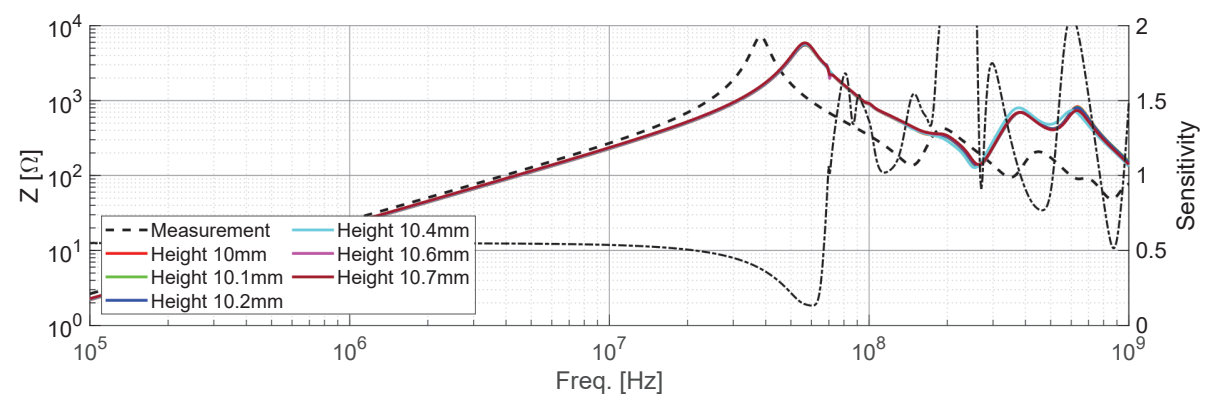

(b) Height

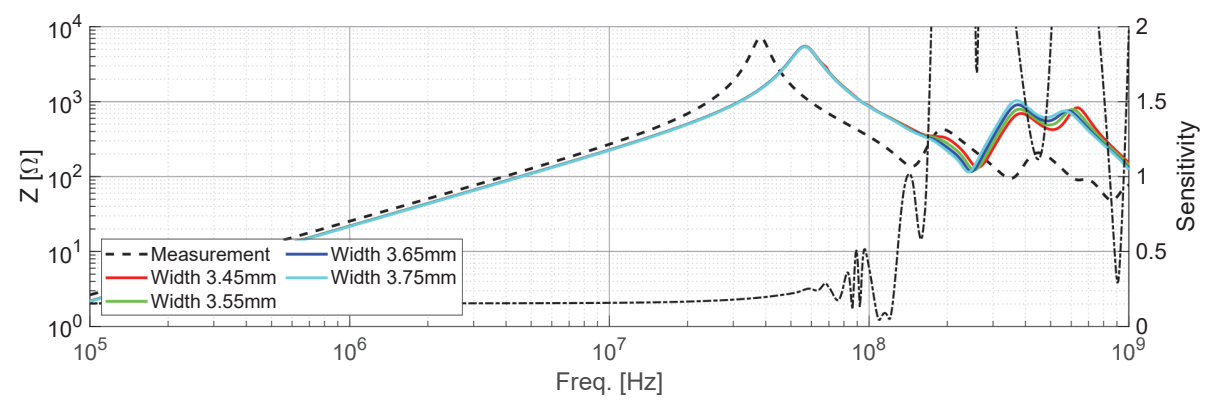

(c) Core width

Figure 4.28: DM impedance. 


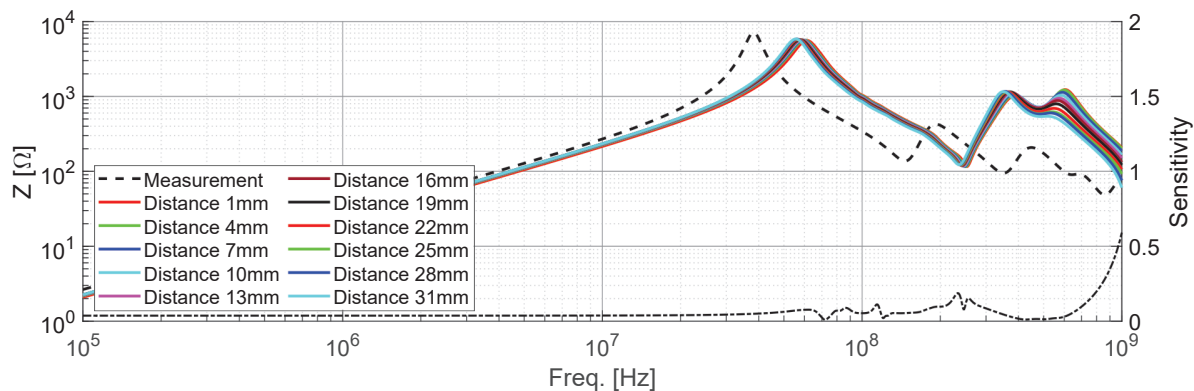

(d) Height above GND

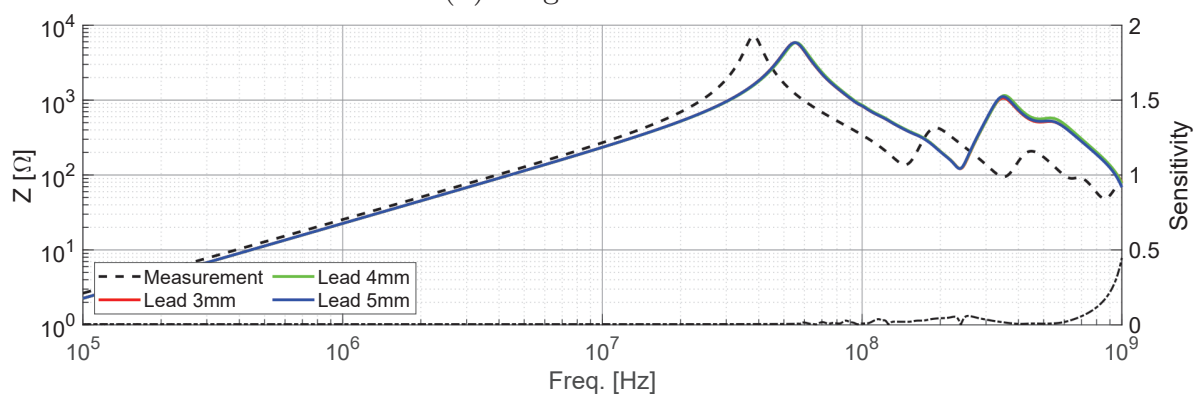

(e) Lead length

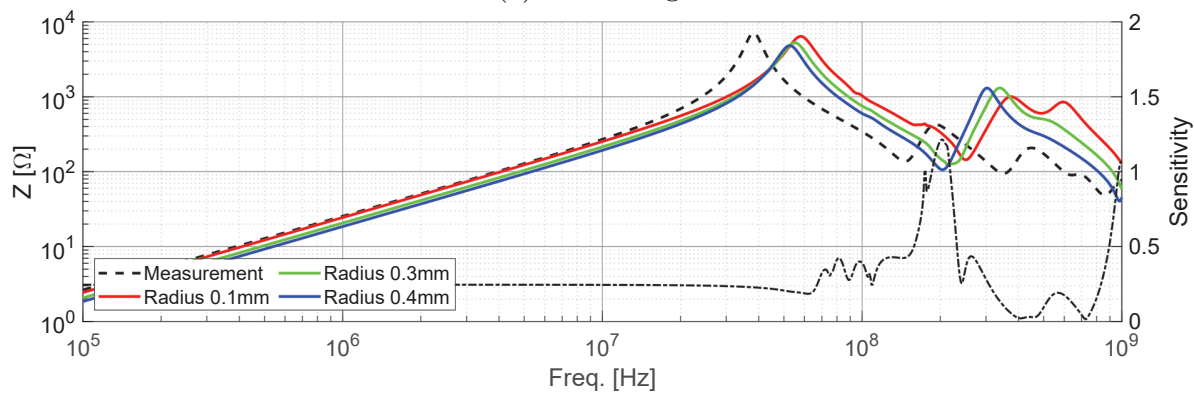

(f) Wire radius

Figure 4.28: DM impedance. 


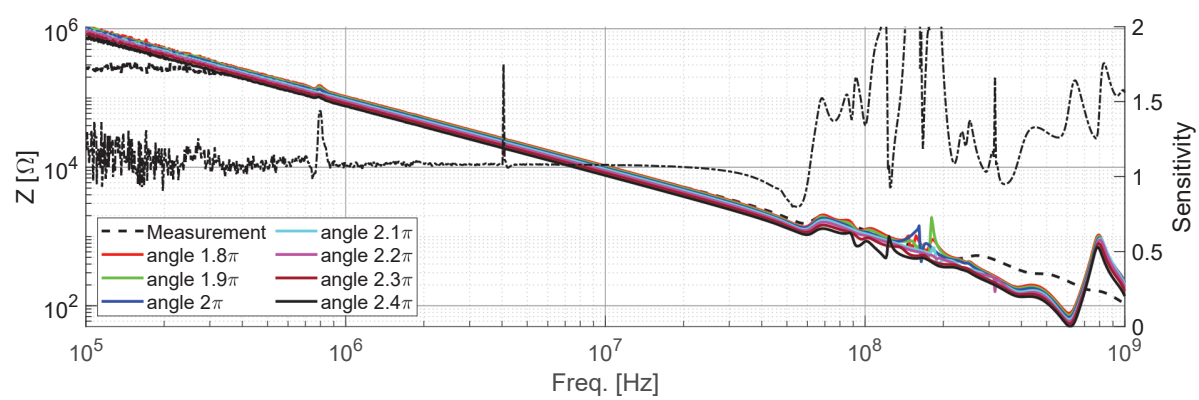

(a) Angle

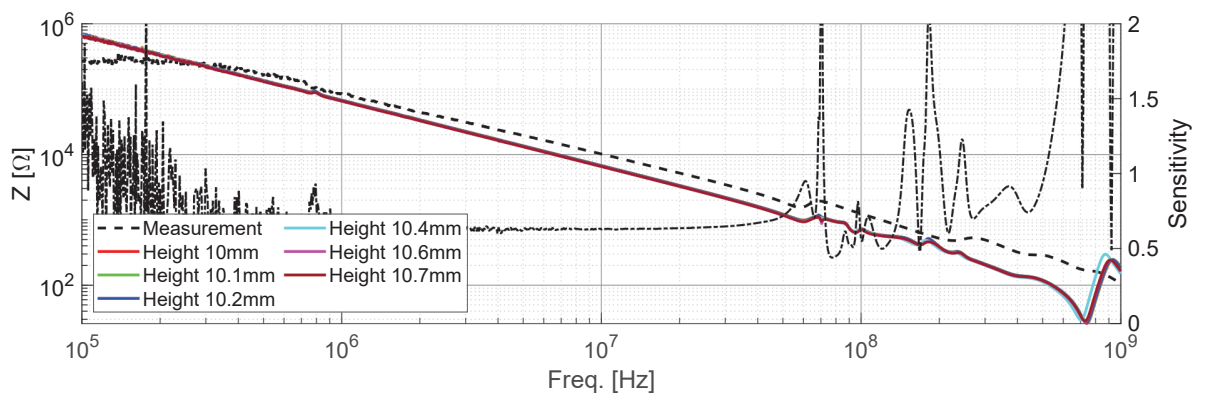

(b) Height

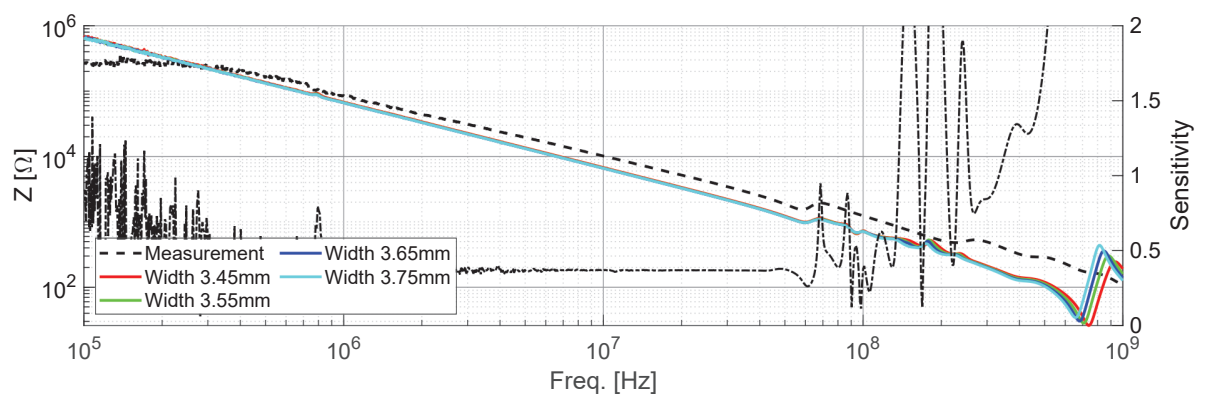

(c) Core width

Figure 4.29: Interwinding impedance. 


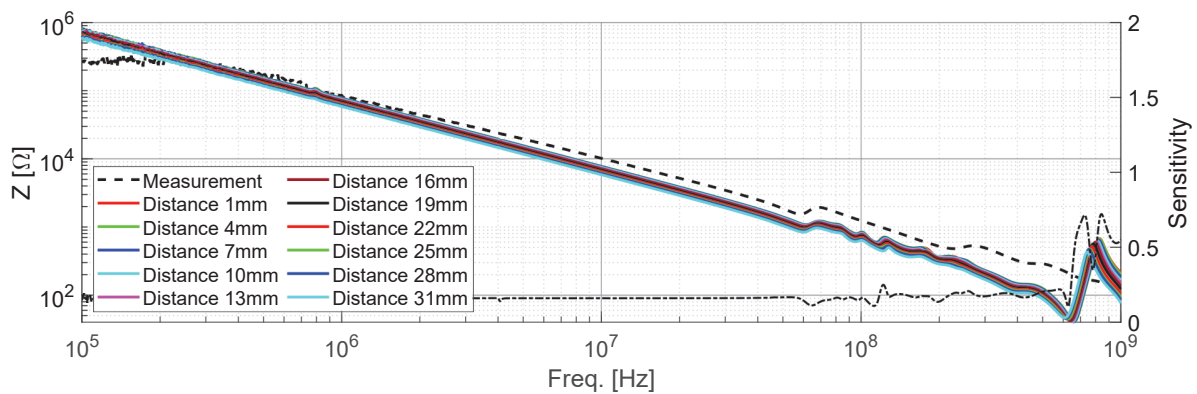

(d) Height above GND

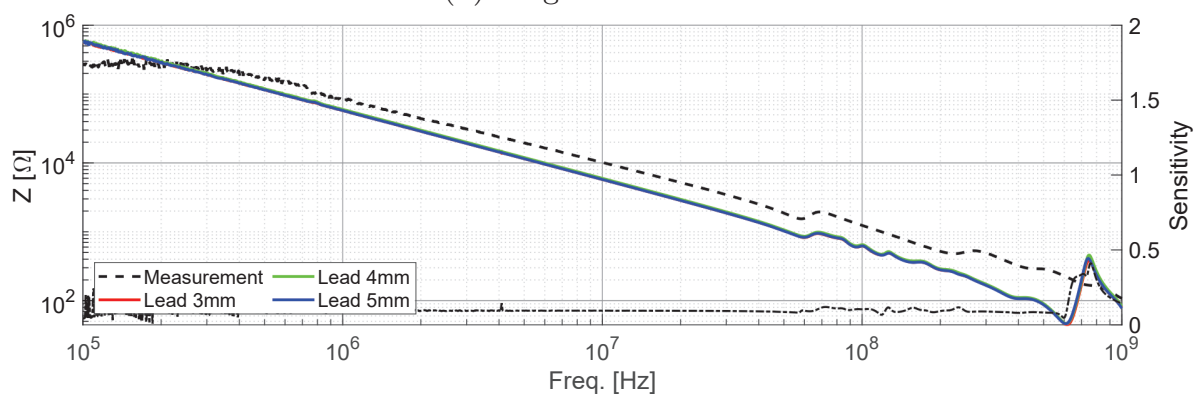

(e) Lead length

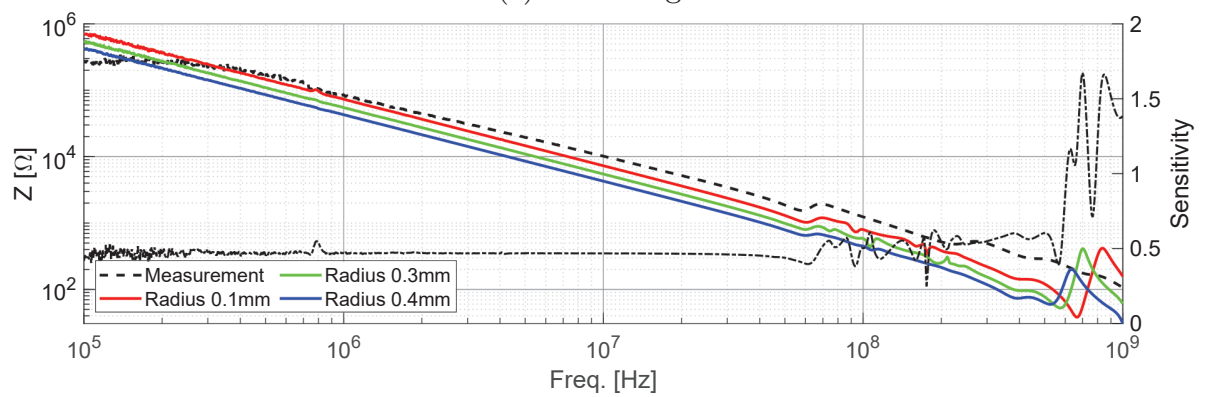

(f) Wire radius

Figure 4.29: Interwinding impedance. 


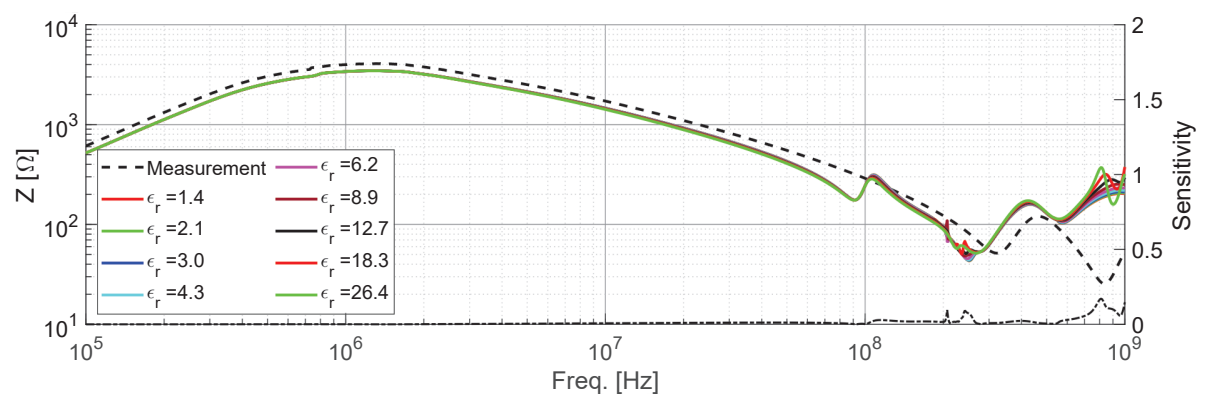

(a) $\mathrm{CM}$

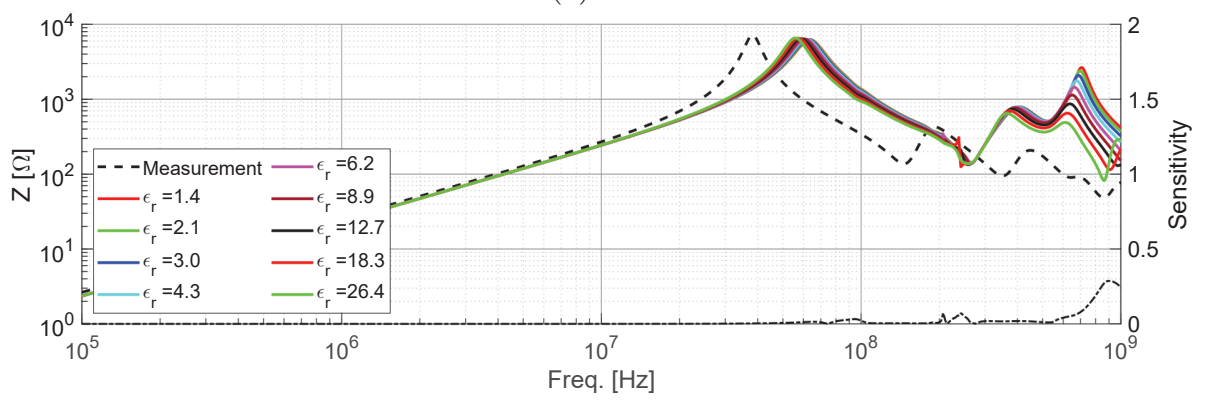

(b) DM

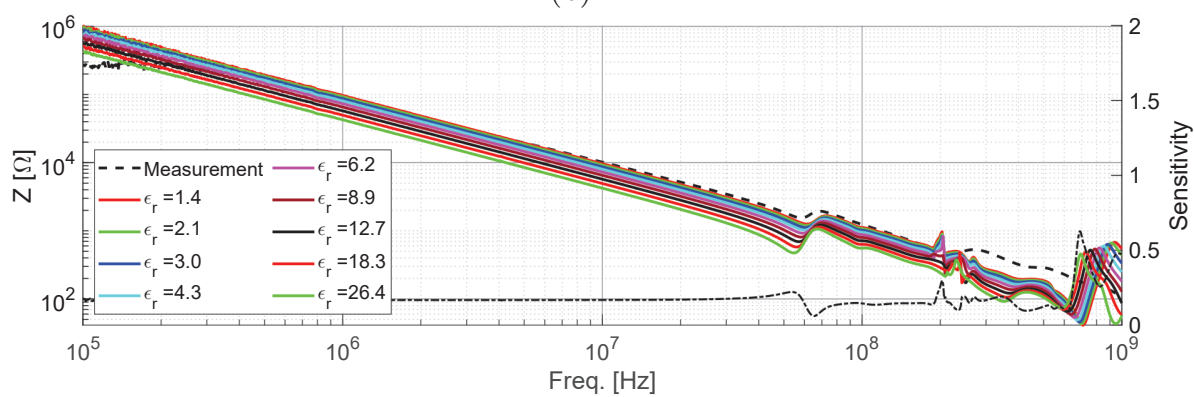

(c) Interwinding

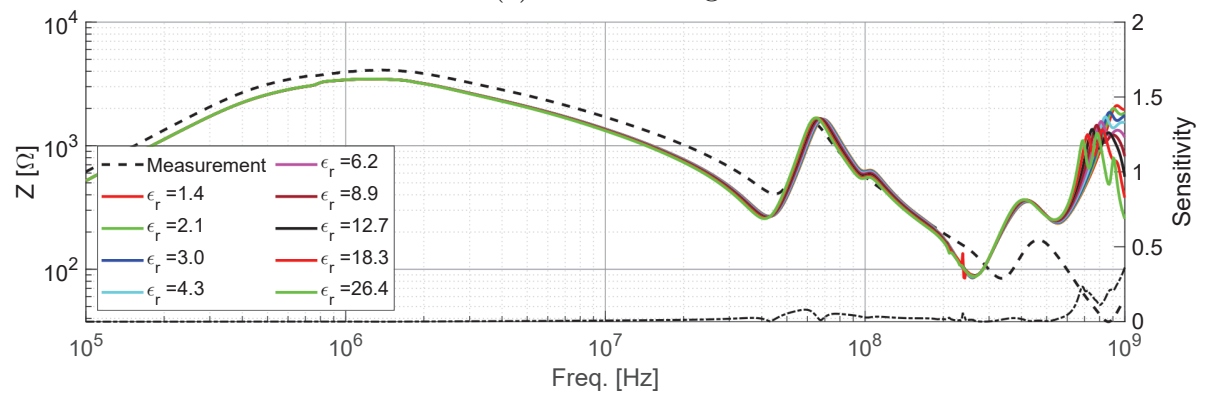

(d) Phase

Figure 4.30: Permittivity influence on the different types of impedance. 
This section has shown the possibility to model a CMC accurately in 3D simulation environments. This allows for parametric studies on parasitic behavior, as well as in-situ performance optimizations within minutes to hours. Compared to measurements with trial and error design strategies this is a significant improvement. Using an automated 3D model creation will also allow for in-situ testing multiple chokes within minutes. The possibilities of investigating hard to control parameters using 3D models have been made apparent in this paper. Specifically the effects on CMC behavior have been investigated and presented. Table 4.4 summarizes the effect simulation parameters have on the resulting impedances of the modeled choke. In this paper the most critical simulation design parameters were identified as quite obviously being magnetic permeability, amount of windings but also the thickness of the wire and the core size. The effect of angle of winding is seen in the parasitic behavior in the form of leakage inductance and inter-winding capacitance.

\subsection{Layout Optimization}

Previous section has shown field effects can be accurately investigated using 3D simulation software. In the following subsections, 3D simulations are used to identify different stray coupling effects on EMI filter transfer functions and back annotated into circuit simulations. The goal is to achieve component placement optimization in an EMI filter, however in 3D simulators the computational time will increase drastically with the amount of coupling parameters that are being included. To overcome this problem, it is suggested to back annotate the mutual coupling impedance into equivalent circuit simulators and then use fast optimization algorithms to discover near optimal solutions with respect to best filtering performance, but optimal with respect to the trade-off of speed and costs versus performance. In this case the mutual induction between two components is modeled in CST microwave studio, while its field effect results are incorporated multiple times in a LTSPICE simulation.

\subsubsection{Case Study}

\section{EMI Filter}

The studied filter topology is a П-filter with the leakage inductance acting as a differential mode inductance separating the two stages of differential mode capacitors. The filter reduces DM and CM currents simultaneously, i.e. it is an integrated EMI filter. The equivalent circuit of the filter is shown in Figure 4.31a. It is based on the common EMI filter topologies described in [15]. These type of filters can be applied for instance in switch mode power supplies. Focus in the design process is on the inter component coupling and the back annotation of these field parameters into SPICE models. The back annotation optimization strategy has been applied to the DM part of the filter. Figure 4.31a shows not only the mutual inductive couplings between the leakage 
inductance and capacitors, but also the mutual coupling inside the CMC core.

Figure 4.32 shows the implementation of the filter, while the equivalent DM and CM filter circuits are shown respectively in Figure 4.31b and Figure 4.31c. As is often the case, the design process splits the CM- and DM- filter design under the assumption of DM-components not effecting the performance of CMcomponents and vice versa. Here the effect of leakage inductance of the CMC coupling into the DM capacitors is shown to degrade performance.

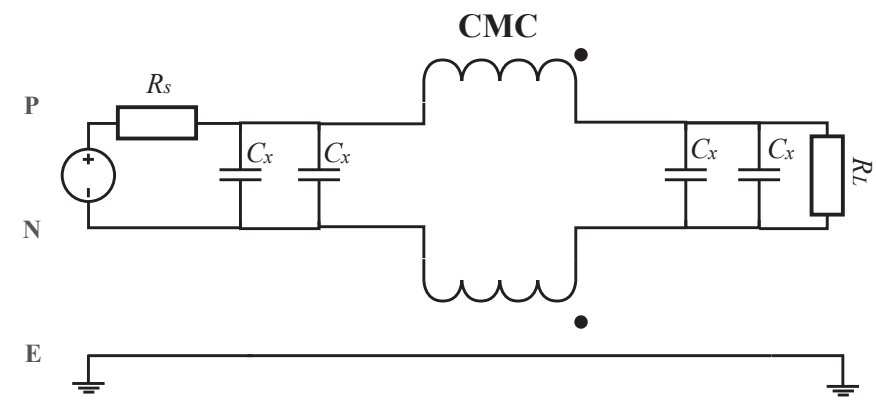

(a) EMI filter with Phase, Neutral and Protective Earth

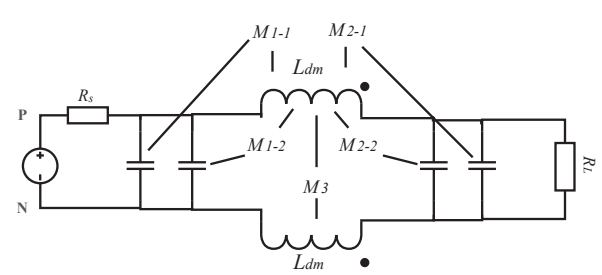

(b) DM decomposed filter

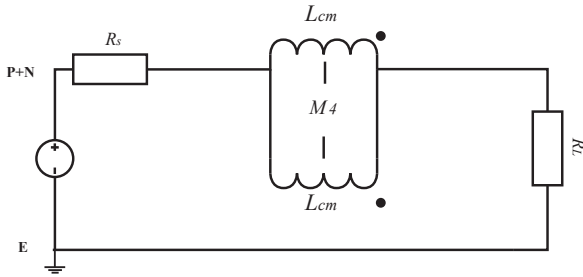

(c) CM decomposed filter

Figure 4.31: Integrated EMI filter, showing internal inductive couplings.

First the basic design of the filter will be shown. An analytical approximation for coupling will be given, to estimate the possible influence. As the influence was considered to be possibly significant, a 3D simulation for mutual coupling extraction was done. The filters performance was then investigated using back annotation of the mutual inductance parameter into LTSPICE. In conclusion, the simulation results are compared to measurements. The placement/orientation optimization is done manually in this case, due to the limited amount of configurations. In case of more complex design filters, this process should be automated as the number of coupling parameters increase.

The optimization is applied to the inter component coupling between the $\mathrm{DM}$ inductance of the CMC and shunt capacitors. In this particular filter it shows that optimizing the layout, the insertion loss in DM can increase with approx. 15-20 dB. 


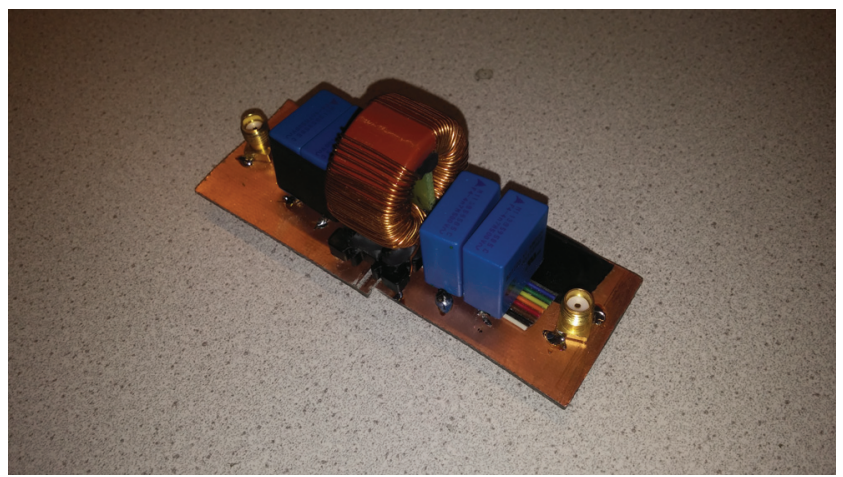

Figure 4.32: Photo of Measured EMI-Filter.

\section{Coupling}

The studied integrated EMI-filter and its DM/CM equivalent circuits are shown in Figure 4.31. The two stages of the differential mode filter are separated by a common mode choke. The DM and CM impedance are split and both have their own parasitic model. For simplicity, here only an inductor with parallel capacitance and resistance were used and not a full behavioral model. To determine the component value and the parasitic values, the impedance measurement was done with the Keysight E5061B Enhanced Network Analyzer (ENA) as was shown in the beginning of this Chapter and further elaborated in Appendix B. Figure 4.14 shows the two configuration in which the CMC was measured. The results are shown in Figure 4.33a. From this the following parasitic values were obtained :

$$
\begin{gathered}
L_{d m}=112 \mu \mathrm{H}, \quad C_{p, d m}=10 \mathrm{pF} \quad R_{p, d m}=370 \mathrm{k} \Omega \\
L_{c m}=18 \mathrm{mH}, \quad C_{p, c m}=45 \mathrm{pF}, \quad R_{p, c m}=15 \mathrm{k} \Omega
\end{gathered}
$$

The EMI-filter has two stages with two parallel capacitors which are identical and placed as $C_{x}$ capacitors. To determine their parasitic ESL and ESR values, an similar impedance measurement was performed using the Keysight E5061B ENA. From this measurement the following values were obtained:

$$
C_{x}=4.7 \mathrm{nF}, \quad E S L=8.2 \mathrm{nH}, \quad E S R=0.141 \Omega
$$

In Figure 4.31 four coupling paths are distinguished, while the mutual coupling between capacitors are neglected. This is based on the results of [58]. The mutual inductance between adjacent capacitors is $1.6 \mathrm{nH}$, which has a relatively small impact on the high frequency behavior of the overall filter. The four coupling paths are split into subcategories. The distinction is made between intra and inter component coupling effects. The coupling between the phases of the $\mathrm{CMC}$ is considered intra, while CMC to capacitor coupling is inter-component. 


\section{Intra-component}

$M_{3}$ and $M_{4}$ are not modeled as separate mutual coupling impedances, as they are intra component coupling paths. By measuring the $L_{d m}$ and $L_{c m}$ of CMC as shown in Figure 4.14, the coupling is already included. Therefore the "physical" placement in SPICE (Figure 4.35) of $M_{3}$ and $M_{4}$ is omitted.

\section{Inter-component}

$M_{1-1}, M_{1-2}, M_{2-1}$ and $M_{2-2}$ are the inductive coupling paths between the $\mathrm{CMC}$ and the four shunt capacitors. This mechanism can be seen in the upper circuit of Figure 4.34a. In the bottom it is shown how the values of inductors would change by incorporating the mutual inductive coupling between the two components. The mutual inductive coupling values are approximated by:

$$
M=k \cdot \sqrt{L_{1} \cdot L_{2}}
$$

With $M$ being the mutual inductive coupling, $k$ the coupling factor, $L_{1}$ being the leakage inductance and $L_{2}$ the equivalent series inductance of the capacitors. From the impedance characteristic of the CMC shown in Figure 4.33a, it can be seen that $L_{1}=L_{D M}=108 \mu \mathrm{H}$ and from [58] follows that $L_{2}=E S L=19 \mathrm{nH}$.

The coupling factor $k$ shows the percentage of flux produced by one component is absorbed by the second component. It is highly dependent on placement distance and orientation of the components. Assuming a coupling of $10 \%$ will result in a mutual induction of $M=145 \mathrm{nH}$. As the high frequency filter performance degradation can often be attributed to a large inductance to the ground connection [13,61], the coupling would drastically increase the induction to ground. Even if the coupling factor would be $1 \%$ it would still approximately double the induction to ground. In most cases, this coupling would have been neglected due to the low DM inductance of the CMC. To determine the impact on filter performance, the filter is simulated with LTSPICE as shown in Figure 4.35 .

\subsubsection{Simulations}

\section{Circuit Simulations}

A distinction is made between the mutual inductances $M_{i-1}$ and $M_{j-2}$, with $i, j \in\{1,2\}$, according to the physical distance to the CMC. In case of the capacitors placed closest to the CMC, in Figure $4.31 \mathrm{~b}$ the capacitors with inductive coupling $M_{1-2}$ and $M_{2-2}$, the distance is approximately $3 \mathrm{~mm}$. For the outer capacitors the distance is approximately $12 \mathrm{~mm}$. A coupling of $10 \%$ is assumed for $M_{j-2}$ and $5 \%$ for $M_{i-1}$. This is based on the orientation and placement of the components shown in Figure 4.32. For simplicity, only the connection schemes shown in Figure 4.36 are considered and three coupling configurations are investigated: 


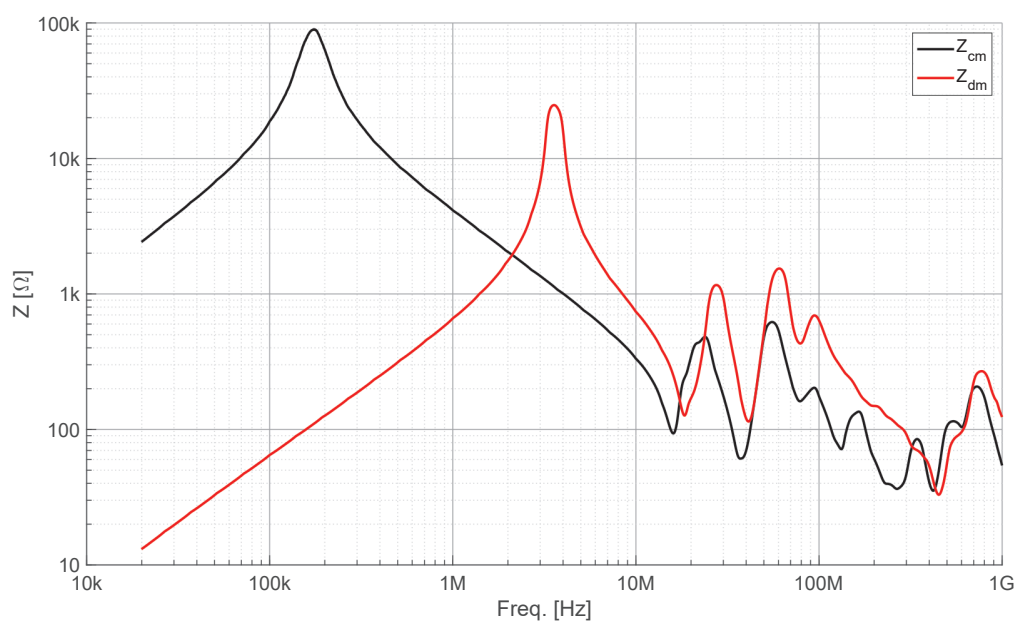

(a) Measured impedance

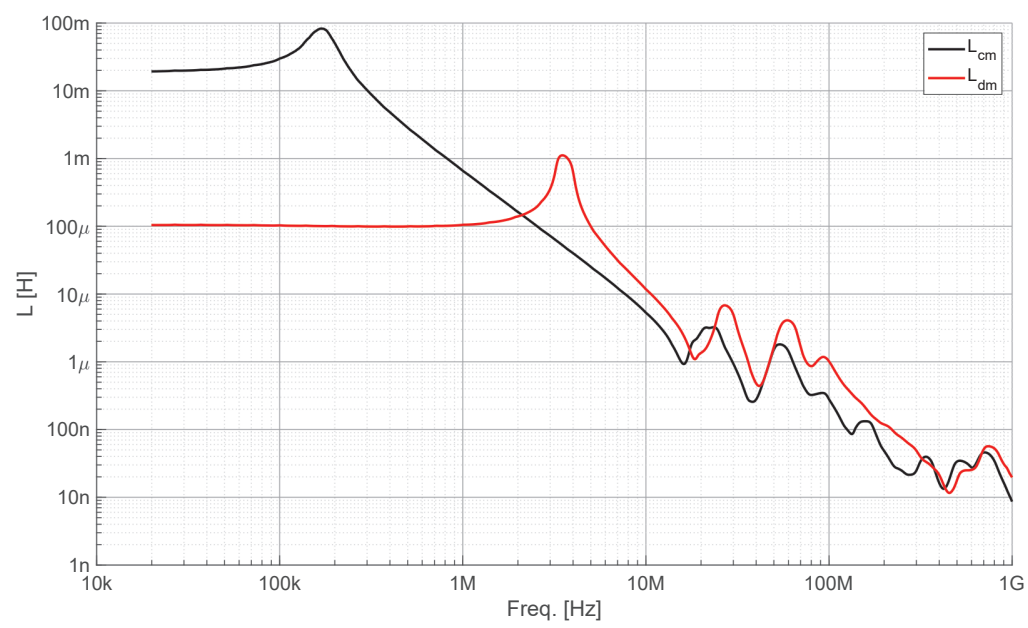

(b) Calculated Inductance

Figure 4.33: Measured DM and CM impedance of the CMC. Calculation has been done by $L=\frac{|Z|}{j \cdot \omega}$. 

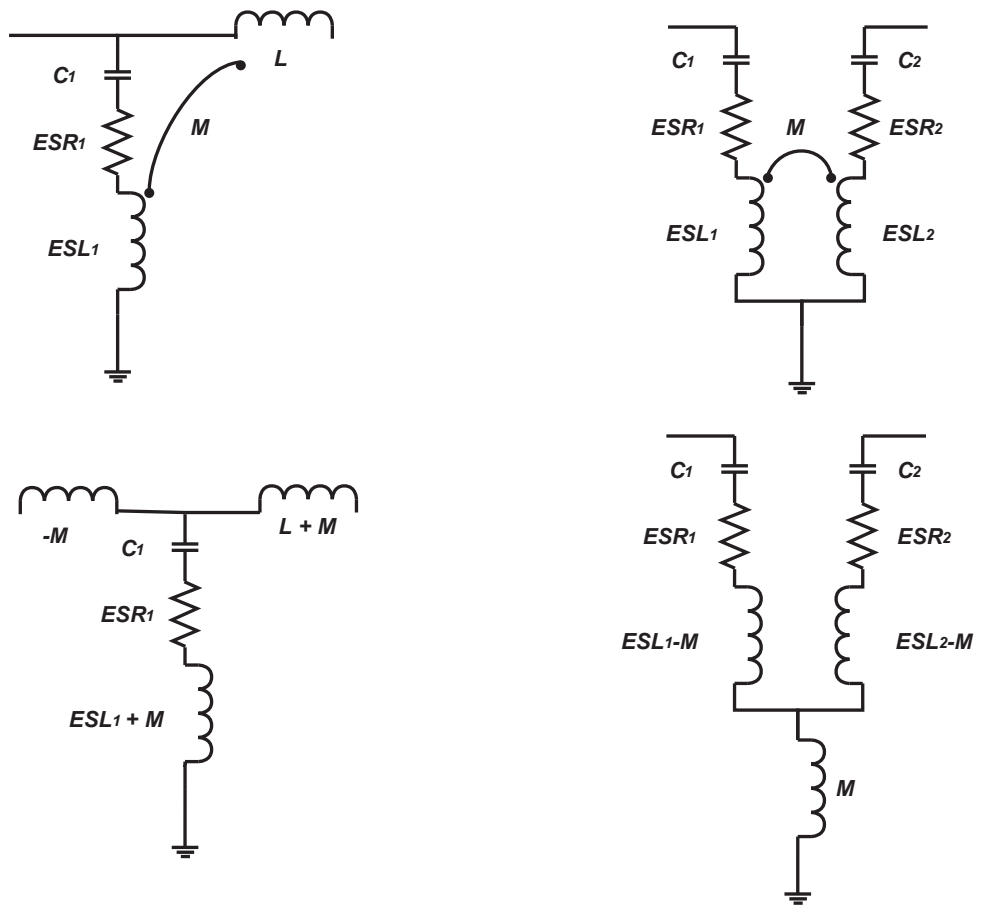

(a) Inductor to capacitor

(b) Two parallel capacitors

Figure 4.34: Equivalent circuits for inductive coupling mechanisms as were shown in [89]. The capacitors are non-ideally represented.

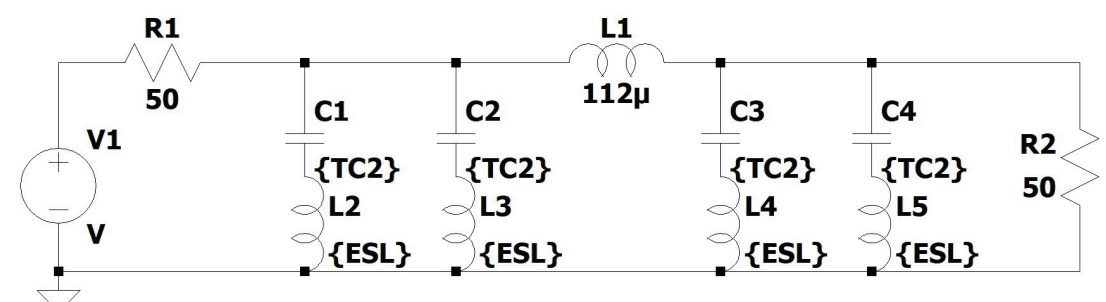

Figure 4.35: Spice equivalent circuit diagram of the filter used to investigate the coupling. 
a) Parallel, which is the conventional type, where all generated currents are in phase.

b) Cross-symmetric, is an alternative type, where the configuration is I-O, O-I. Therefor is symmetric around the CMC

c) Cross-asymmetric is an alternative type, where the configuration is I-O, $\mathrm{I}-\mathrm{O}$. Therefor is asymmetric around the $\mathrm{CMC}$

The distinction is made based on the current being generated in the capacitors either to be in-(I) or out-of phase $(\mathrm{O})$ compared to the first capacitor on the left hand side of the filter.

a)

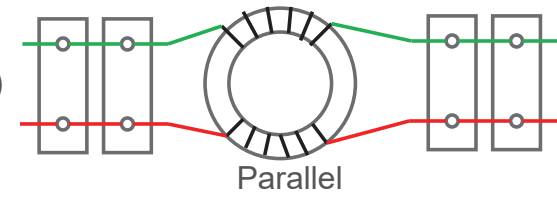

b)

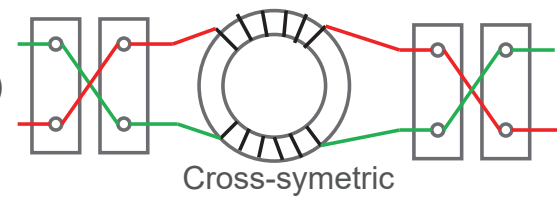

c)

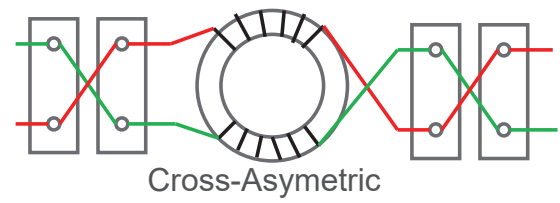

Figure 4.36: Different coupling configurations.

These are shown in Figure 4.36. Crossing the phase and neutral between two parallel capacitors is an idea described in [58]. It creates a negative coupling between the capacitors and results in a reduction of the combined ESL. The mutual coupling between CMC and two capacitors should also be reduced. When in parallel, the currents generated in one capacitor add constructively to the currents in the adjacent one. When using this crossing method, the currents are $180^{\circ}$ out of phase.

Figure 4.37 shows a theoretical improvement of $15-20 \mathrm{~dB}$. Note however this is assuming the coupling factor to be $5-10 \%$. To confirm the approximation a $3 \mathrm{D}$ simulation was created in CST Microwave studio. This is explained in the following section.

\section{Field simulation}

The 3D EM-field simulation has been carried out using the simplified inductor model shown in [90] and the simplified capacitor model described in [68]. The 


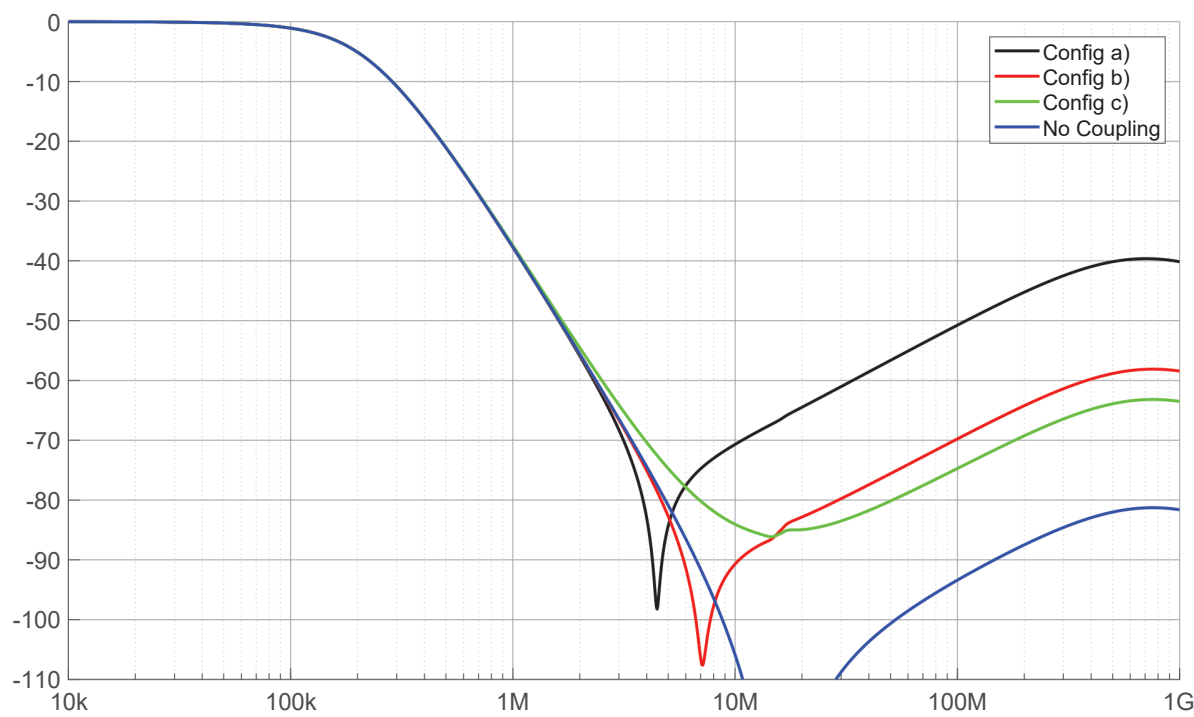

Figure 4.37: The theoretical improvement gained by using a cross-over of phase and neutral between capacitors in individual stages of the filter.

models are shown in Figure 4.38b. Since the focus is on back annotation, only the resulting mutual inductance will be presented here. Which was derived from Equation 4.24 in [62].

$$
M=\left|\frac{2 \cdot Z_{0} \cdot S_{21}}{\left(1-S_{22}+S_{22} S_{11}-S_{11}-S_{21}^{2}\right)}\right| \cdot \frac{1}{(2 \pi f)} \approx 90 \mathrm{nH}
$$

This shows the coupling to be in the order of magnitude of ESL and therefore a limiting factor to the filter performance. Next the multiple configurations are measured and compared to the SPICE simulations.

\section{Verification of 3D Simulation}

Measurements were done to verify the results from the 3D EM-field simulation. The S-parameters were measured between the actual used capacitor and CMC using the Keysight ENA. Figure 4.38a shows the setup used. The capacitor was positioned at a similar distance as it is going to be implemented. The mutual inductance is then calculated through Equation 4.24 and results in:

$$
M_{i-1}=90.3 \mathrm{nH} \quad M_{j-2}=56.1 \mathrm{nH}
$$

\section{Back Annotation}

To back annotate the 3D EM-field parameter of mutual inductance in the used circuit simulator, the coupling factor needs to be determined. By rearranging Equation 4.23, the coupling factor can be determined based on the self 


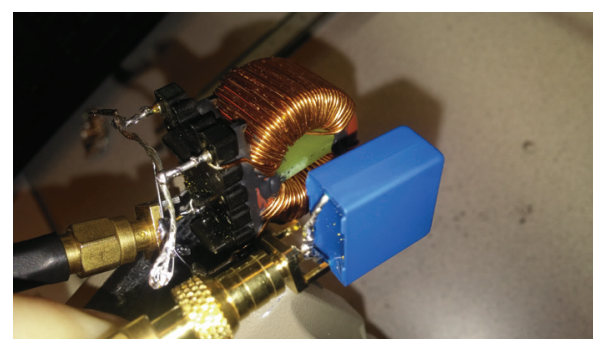

(a) Measurement for verification

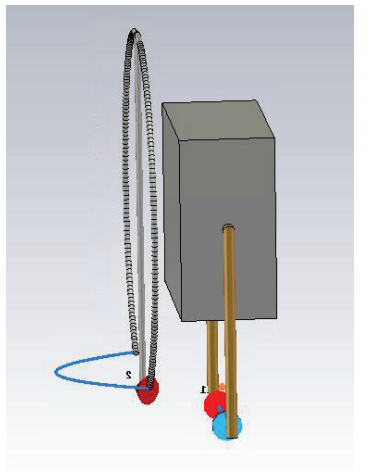

(b) Simplified 3D EM-field simulation

Figure 4.38: Determining mutual inductive coupling.

inductances of the components and the mutual inductance between them. To determine the coupling factors value the following equation is used:

$$
k=\frac{M}{\sqrt{L_{1} \cdot L_{2}}}
$$

Using the results of the two mutual inductances together with the values of $E S L$ and $L_{d m}$, the coupling factors were found to be $k_{1} \approx 0.1$ and $k_{2} \approx$ 0.06 respectively. By implementing these in the circuit simulation (shown in Figure 4.35) an appropriate component orientation can now be chosen based on the filter requirements.

\subsubsection{Filter Measurements}

The back annotation of DM driven CMC to capacitor coupling suggests it is possible to increase the high frequency attenuation (by 15-20 dB) of a common EMI filter topology by choosing a more optimized connection of components. This is a noticeable achievement for two reasons. Firstly, the $L_{d m}$ is often neglected, and thus the coupling from this DM inductance to other components as well. Secondly, the optimized connection scheme was found using equivalent circuit simulations. The circuit simulations were verified by measuring the configurations using a VNA. The configurations were all implemented on the same PCB, using the same exact components. The PCB consists of four electrically separated conducting planes in which the topside (shown in Figure 4.32) is used as neutral and bottom side as phase. The two planes on either side of the board are only connected through the CMC (and VNA). The capacitors are then alternately connected as shown in Figure 4.39 

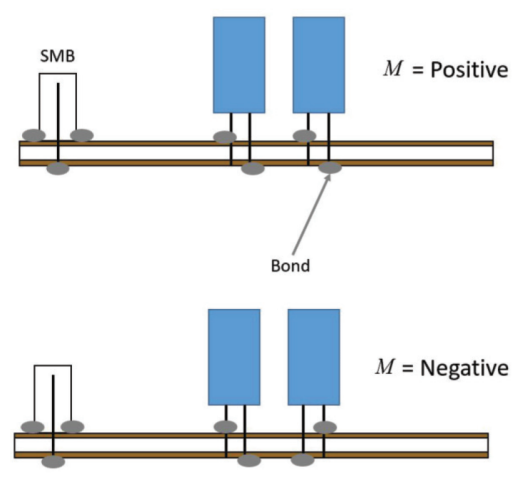

Figure 4.39: Sketch of a section of the measurement setup that shows how to inverse the magnetic coupling.

\subsubsection{Results}

The measurements are shown in Figure 4.40. As was predicted, the coupling between CMC and capacitors severely degrades the high frequency performance of the EMI filter. However an optimal placement of components found via back annotating the 3D EM-field coupling parameters increases the performance with approximately $15 \mathrm{~dB}$ from $5 \mathrm{MHz}$ to $200 \mathrm{MHz}$.

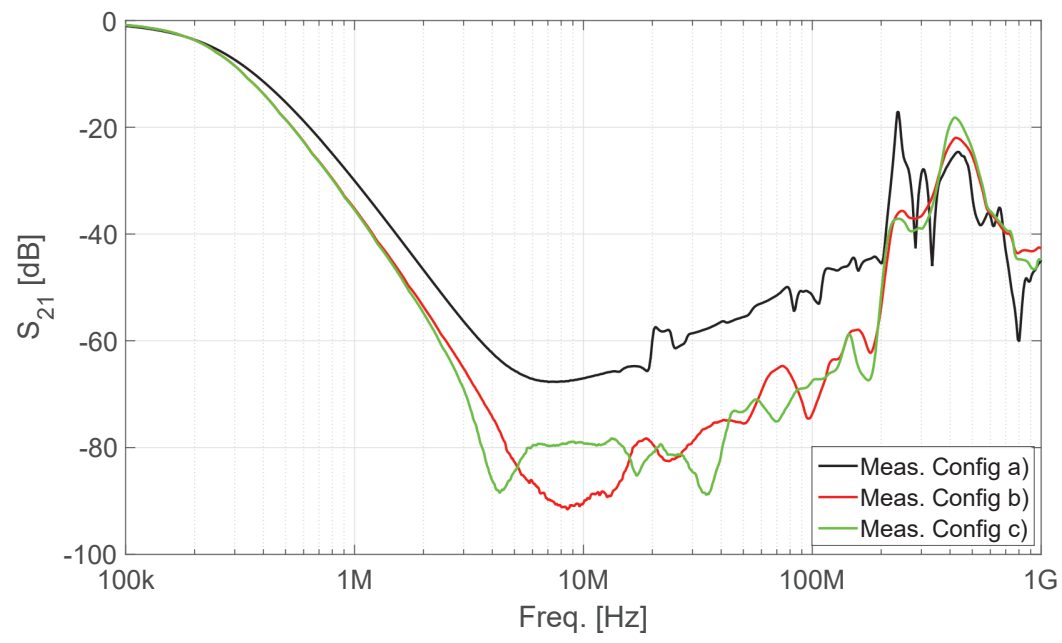

Figure 4.40: DM - Measurement of filter.

Based on a simple approximation of the inductive coupling between components it was suggested that through optimized component placement an improvement of $15 \mathrm{~dB}$ or more could be achieved for frequencies between $5 \mathrm{MHz}$ 
and $200 \mathrm{MHz}$ for this specific passive filter. The approximation was verified by $3 \mathrm{D}$ EM-field simulations and the mutual inductance parameter was back annotated into circuit simulations. It showed possibilities for high frequency performance improvement. Measuring multiple component orientations of the same implemented filter verified the expectations based on the circuit simulations. It has been shown that mutual coupling between components are also a limiting factor in high quality passive filters. Therefore the understanding of these coupling mechanisms and their modeling should be encouraged. It has also been shown that the connection to ground, or safety earth in case of CM, is determining the performance degradation at high frequencies. The parasitic induction of a capacitor plus the inductive coupling into a capacitor is often the limiting factor. By using coaxial feed through capacitors the loop of the capacitor its input to output is minimized, i.e. the equivalent series induction is minimized [13].

\subsection{Summary}

At the beginning of this chapter an algorithm has been developed for extracting equivalent circuits from impedance curves based on a Gauss-Newton optimization scheme. It has been applied to individual measurements of components used in CM filter and the measured mutual coupling between these components. The algorithm searches for optimal values for an X-amount of RLC-components in a predefined equivalent circuit. It was shown that it will increase the simulation results accuracy. The development of this algorithm was a necessity for automated back annotation of fields into circuit simulators.

As the CMC is one of the most important parts of any EMI filter, the second part of this chapter has described the design of a 3D full wave model for high frequency $(100 \mathrm{kHz}$ to $1 \mathrm{GHz})$ simulations. Many researchers have investigated and developed modeling techniques for several passive components e.g. filmcapacitors, toroidal inductors, surface mounted capacitors and inductors. Due to the complexity of the $\mathrm{CMC}$, it was required to develop this $3 \mathrm{D}$ modeling technique for eventual back annotation of coupling fields.

The third and last part of this chapter describes the technique to include mutual inductive coupling into equivalent circuit simulations and how it can be used to investigate optimal component placement in filters. It has been shown that the filter performance improved with $15 \mathrm{~dB}$ between $5 \mathrm{MHz}$ and $200 \mathrm{MHz}$.

Combining the three techniques shows a method that can automatically back annotate a field effect between components and optimize filter design based on the placement and thus utilizing the field effects, without compromising the speed of the design and the required skill and expertise necessary. This can be considered to be the major contribution of this chapter to the thesis. It implies that designing more optimal filters is possible for engineers with limited expert knowledge, which is of great practical value to EMC, power electronic engineers or anyone designing filters. 


\section{Chapter 5}

\section{EMI Mitigation through EMI Placement}

The development principle of conventional Switch Mode Power Supply (SMPS) is aimed at reducing weight and cost, while delivering a maximum achievable efficiency. In general, this translates into implementing faster semiconductor switches, in order to reduce dissipation losses. This, in turn, leads to high amounts of harmonic distortion [91] and generated EMI. Mitigation techniques have been studied extensively in a wide range of possibilities and application levels.

The application of the M3C topology is envisioned for a HVDC network. Traditional mitigation methods are often costly and require specific development. The $\mathrm{M} 3 \mathrm{C}$ is consisting of multiple sub-modules, which in principle are two semiconductor devices in a half-bridge formation.

This chapter addresses possible mitigation techniques for conducted as well as radiated EMI. It will follow a similar approach as shown in Chapter 2. First the conducted EMI will be investigated and one of its most common mitigation techniques 'filtering'. Previous chapter has shown some techniques that could help improve overall filter performance by assisting in the design phase through accurate high frequency modeling techniques. In the first part of this chapter, it will be shown that EMI can be shifted in frequency without compromising the functional behavior of the system. It can then be applied to carefully place the EMI in regions that a filter will work most optimally. Traditionally one would design the filter to reduce the emission level most optimally, however due to increasing switching frequencies the noise is generated at frequencies in which parasitic behavior of components or even the entire system become more dominant. Instead of trying to define all parasitics and minimize their effects, a more practical approach is suggested to accept them and shift the EMI. Of course a good designer takes care of the parasitics a much as possible, but

Parts of this chapter have been published in [23] and [28]. 
eventually there will some that were overlooked or even that are un-containable and reduce the performance of a filter.

Next to containing conducted EMI, also radiated noise should be addressed. Especially in systems where high dv/dt's and high di/dt's are expected as radiated EMI is a time derivative effect. Chapter 2 has shown a direct relation exists between the SPWM voltage at the output of a SM and the radiated electrical field. The second part of this chapter explores the possibility to create an effective radiation model based on the in-situ operation and the mathematical model derived in Chapter 2. Here it is assumed the entire system acts as some radiating entity, with a known input signal and a measured received signal. By varying the sPWM generation parameters $\left(f_{c}, f_{m}, m_{i}, D_{i}\right.$ and $\left.t_{\text {dead }}\right)$, one is able to sweep the entire spectrum of interest in a similar fashion as a test receiver with tracking generator. The measurement is not a small signal analysis as it is performed under operating conditions. The results are useful for a multitude of reasons. In compliance testing of a Switch Mode Power Supply (SMPS) that is as flexible as the one used in this study, one is unable to test each possible setting without spending an enormous amount of time (and money) in the test facilities. By using the developed technique one can test a limited set of possibilities and estimate the amount radiated EMI for any combination of control parameters. Next to this, it can also be used as a possible EMI mitigation technique that is quite similar to the one described in the first part of the chapter. By determining the transfer function of the system, one inherently identifies the most and least radiative frequencies of the system. By shifting and placing the generated noise to those that are least radiative, one reduces the EMI by optimally using the system characteristics.

The techniques presented in this chapter are focused on reducing EMI, without any regard for some critical design parameters of a SMPS or M3C, e.g. efficiency, energy density, cost of components, reliability, output power, voltage transfer ratio, complexity of control signals. The mitigation proposed is most useful at the integration stage of the converter. i.e. at the moment the complete product is EMC compliance tested in-situ, as all the parasitic components are included and the product might be failing the tests. Theoretically it would not affect the cost of components, output power, voltage transfer ratio, and complexity of control signals. For the effect on efficiency, energy density, and reliability, future studies are needed.

\subsection{Conducted EMI Mitigation - Optimal Filter Us- age}

\subsubsection{Carrier Frequency Shift}

This section investigates the EMI produced in a half-bridge, while mitigation is achieved by using an existing filter optimally by placing most of the EMI generated in its most effective bandwidth. The SMPS consists of two SiC based 
MOSFETS with a $\mathrm{SiC}$ recovery diode in parallel. A commercially available evaluation kit has been used as a DC/AC buck-converter (Figure 5.1).

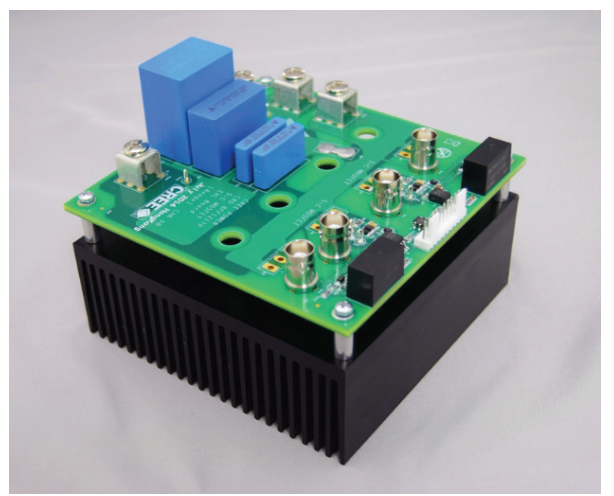

Figure 5.1: SiC based half-bridge

Figure 5.2a shows a schematic representation of the setup and is similar to the $\mathrm{DC} / \mathrm{AC}$ converter studied in Chapter 2. The sPWM signals provided to the driving circuit of the half-bridge is provided through a chain connection depicted in Figure 5.2b which is elaborately explained in Chapter 3. The main contributing frequency components were identified based on the analytical model derived and numerically verified in [22]. The findings are restated here:

Fundamental Frequencies: $f_{m}, f_{c}$

Harmonics: $f_{m} \cdot i, f_{c} \cdot i$

Sub-Harmonics: $f_{c} \cdot i \pm f_{m} \cdot j$

In which $f_{m}$ and $f_{c}$ are considered to be the wanted signal and switching frequency respectively. $i$ and $j$ are positive integer numbers. From this it follows that it is possible to shift the EMI spectrum, without compromising the desired signal. i.e. it is possible to shift the carrier frequency and eventually reduce the peak output EMI. Not by reducing or spreading the generated EMI, but to optimally use the output filter, therefore the mitigation technique investigated is related to the coupling path and not the source.

The system designer is flexible in choosing $f_{m}, f_{c}$ and the output filter. Depending on the design criteria, one chooses a combination best suited for the application. In Figure 5.3 it can be seen that the choice of operating frequencies influences the possible output filter transition band, i.e. it could relax the design constraints of the filter by increasing the gap between desired and unwanted power. In theory this seems like a trivial and easy task, by choosing an as high as possible switching frequency. In that case one neglects the possible decrease in functional behavior, which in case of a SMPS can be efficiency. Next to the functional behavior it has often been the case that unknown parasitics (at the higher frequencies) will compromise the performance or even the service of the system. 
Cree - Eval KIT

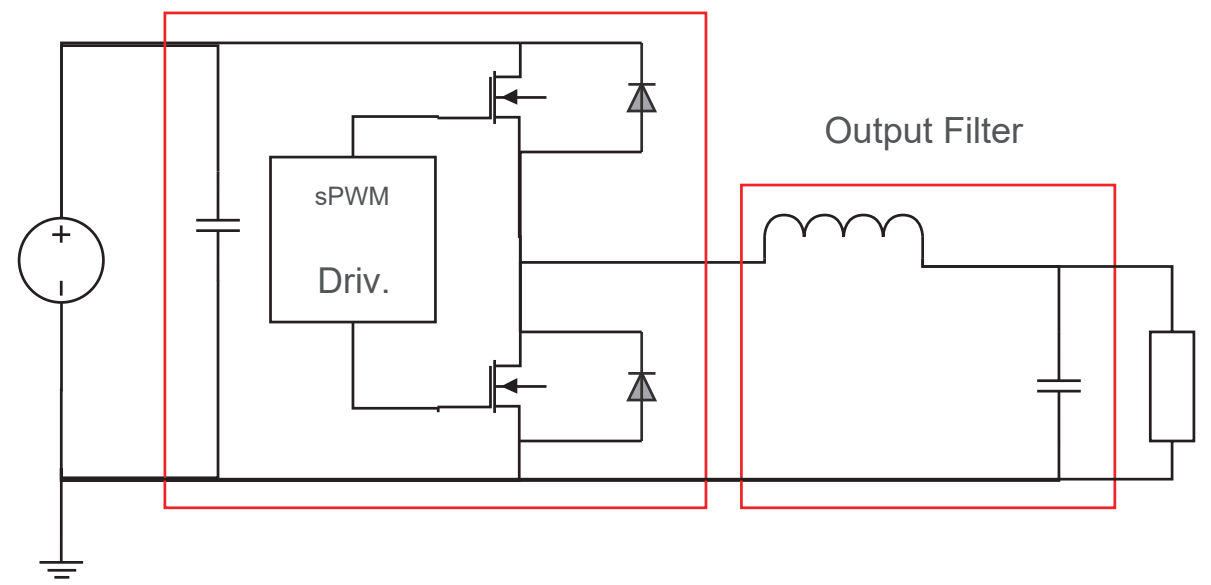

(a) $\mathrm{SiC}$ based half-bridge $\mathrm{DC} / \mathrm{AC}$ converter

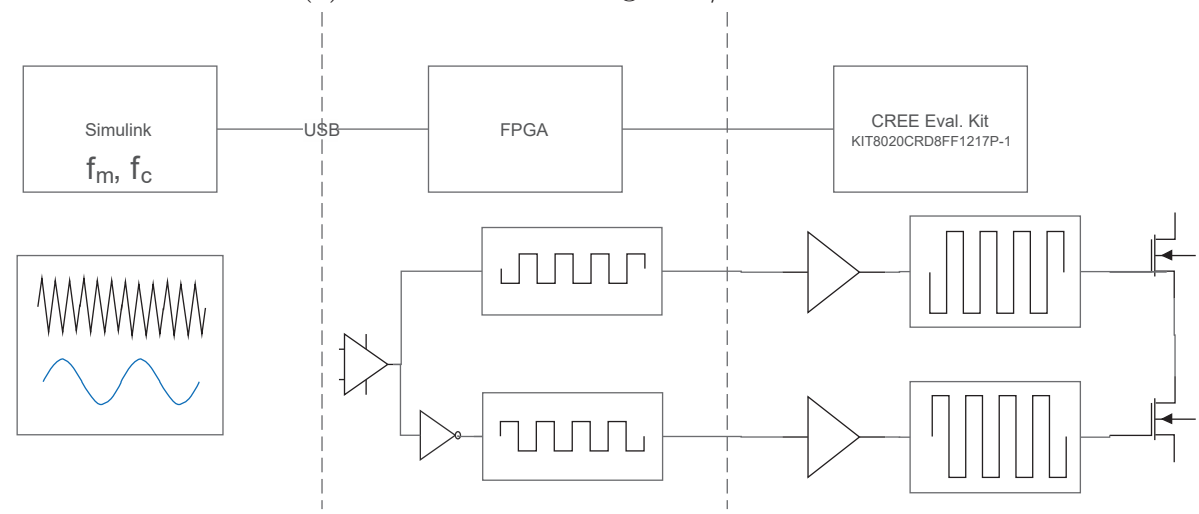

(b) sPWM driver

Figure 5.2: Setup 


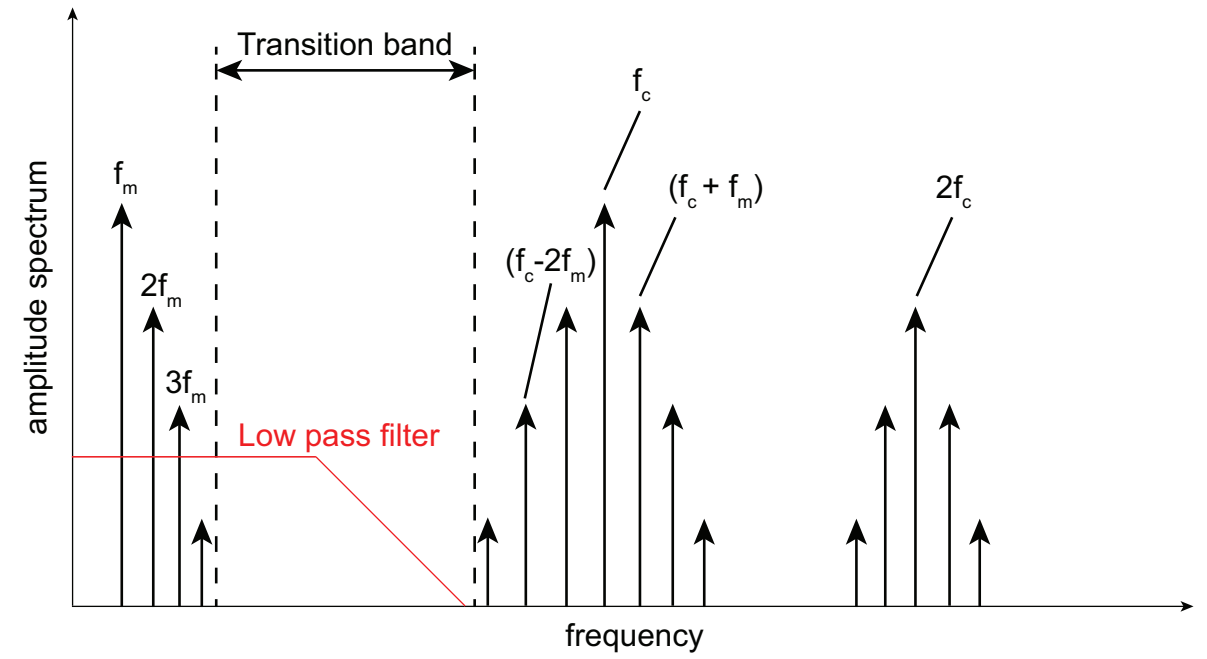

Figure 5.3: Amplitude spectrum of sPWM

In the following sections the implementation of the buck converter is discussed, with the emphasis on carrier/switching frequency shifting and its possible benefits in mitigating EMI.

\subsubsection{Application}

It is clear that $\mathrm{SiC}$ based semiconductor technology combined with FPGA generated sPWM enables new possibilities for power electronics. A key benefit is flexibility based on several design parameters. The power line and carrier frequency can be adapted based on the application. Voltage transfer ratio can be controlled and is related to sPWM generation. Based on output voltage measurements a control loop can be implemented to stabilize this value. Also in case of usage in $\mathrm{MC}$ a Selective Harmonic Elimination (SHE) Model Predictive Control can be implemented [92].

\section{Power Line Frequency $f_{m}$}

This is determined by the application of the converter and can go as high as $100 \mathrm{kHz}$ when needed. Several application areas with different frequency bands are already implemented, e.g.:

- DC - any small household electronic device (phone charger, USB powered devices), high voltage power lines etc.

- 16.7-25 Hz - Railway systems

- $50 / 60 \mathrm{~Hz}$ - Conventional power system 
- $400 \mathrm{~Hz}$ - Airplanes

- $20-100 \mathrm{kHz}$ - Induction heating

- $80-100 \mathrm{kHz}$ - Wireless power transmission

\section{Carrier Frequency $f_{c}$}

The carrier frequency is often chosen to be an order of magnitude larger than the power line frequency, for more accurate waveform creation even higher frequencies are used. The higher the carrier, the smoother/better the resulting waveform. Increasing frequency, can also relax EMI filter requirements. One can trade off cuttoff frequency and filter order. A lower order filter is often cheaper (and smaller), and might be desired. Thus, by increasing the carrier frequency one can save money and space. At increased frequencies, system design can become more complex due to the non-ideal behavior of components. Figure 5.4 shows a sketch of a possible frequency spectrum with a non-ideal filter behavior as an example. Good filter design will increase the bandwidth of the stop-band and/or maximum attenuation. However even good filter designs can fail at integration into a system, as was described in [13]. Given these circumstances here will be shown that given a certain filter one can adjust the carrier frequency such that it and its harmonics are in most effective filter bandwidth. Using the example in Figure 5.4, one can clearly see that the carrier frequency will be attenuated adequately, however due to some unpredictable resonance the first harmonics can propagate almost unattenuated. Shifting the fundamental switching frequency, either lower or higher, might reduce the attenuation of itself but increases the attenuation drastically of the first harmonic. To demonstrate the possible mitigation technique, in the experimental setup a COTS filter was used, knowing it performed poorly at higher frequencies $(>1 \mathrm{MHz})$. Its transfer characteristic can be seen in Figure 5.5. The filter represents a real situation in which a good design fails at the end of the integration process. By shifting the EMI spectrum to the appropriate frequencies, EMI compliance might still be achieved.

This only holds if the designer is truly free to chose the carrier frequency, which is the case if there are no optimal combinations of $f_{c}$ and $f_{m}$. Here it was chosen to use Signal to Noise Ratio (SNR) as figure of merit. As SNR is defined as $S N R=\frac{P_{s}}{P_{n}}$, an increased SNR shows that either the power of EMI is decreased or power of the functional power line frequency is increased. In case of a constant SNR, one can conclude there is no optimal or pessimal choice. A numerical analysis on combinations of power line and carrier frequency is performed. Figure 5.6 shows a contour plot of the SNR at different combinations between $f_{m}$ and $f_{c}$. For $f_{c}>5 f_{m}$ the SNR is approximately constant. At $f_{c}=i \cdot f_{m}$, with $i$ being a positive integer, an increase in SNR can be seen, as a subharmonic of the carrier will coincide with the desired signal and is thus excluded from the calculation of $P_{n}$. 


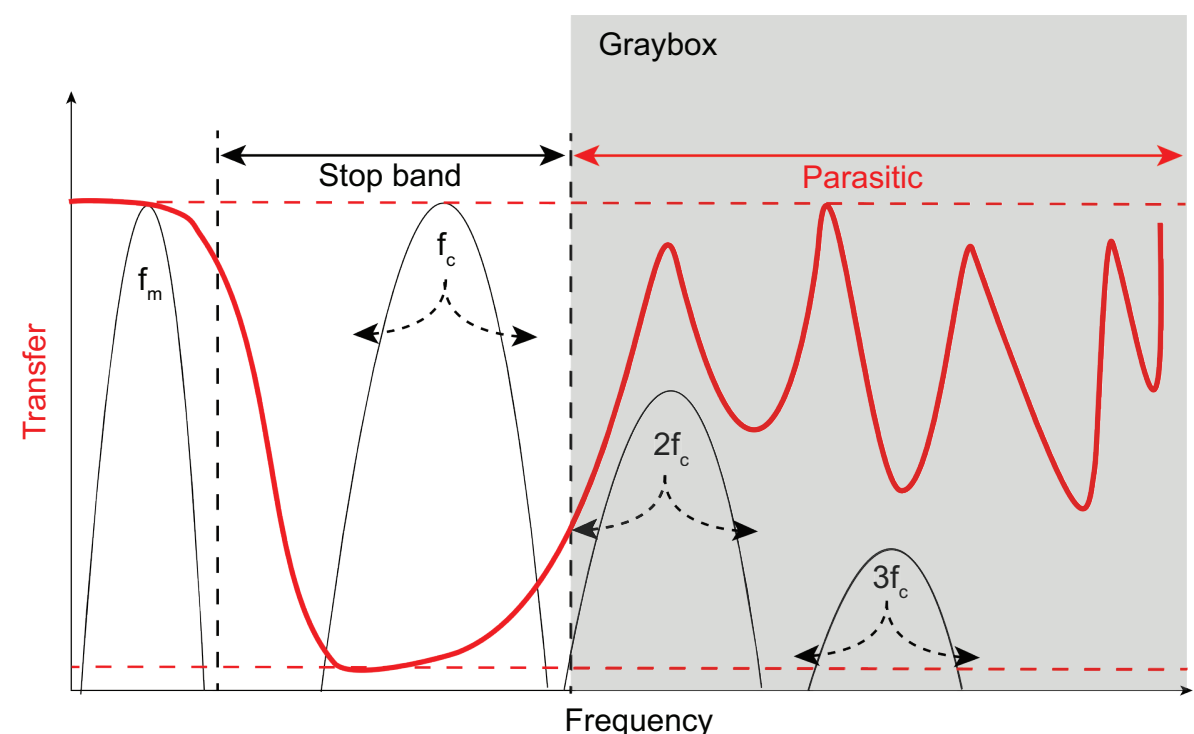

Figure 5.4: Possible transfer characteristic of non-ideal filter while showing the ability to shift carrier frequency into desired bandwidth

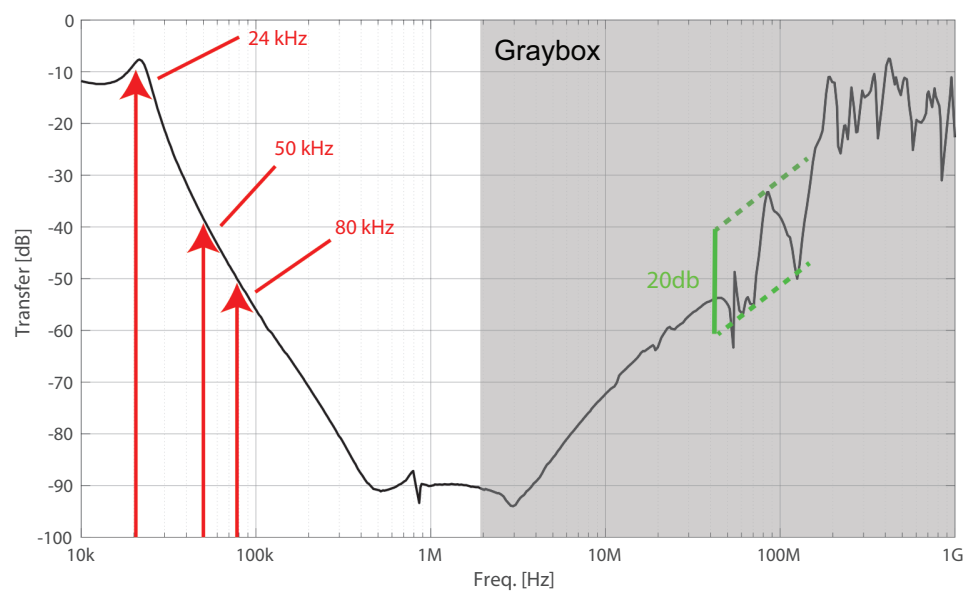

Figure 5.5: Unexpected poor performing filter [13] with chosen carrier frequencies which are limited by hardware. 


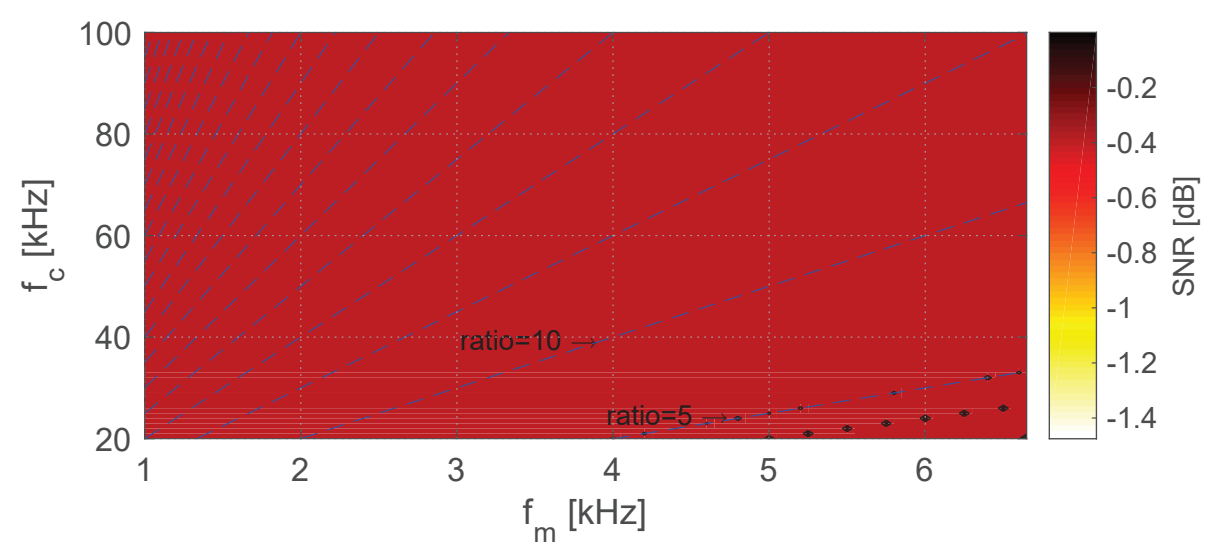

Figure 5.6: Near constant SNR for any combination of modulation and carrier frequency.

Based on the results and filter response shown in Figure 5.5, the EMI of the setup is evaluated at three different carrier frequencies while maintaining the same power line frequency $(2 \mathrm{kHz})$. The frequencies $24 \mathrm{kHz}, 50 \mathrm{kHz}$ and $80 \mathrm{kHz}$ have been chosen, which in theory should be suppressed with approximately 8 , 40 and $50 \mathrm{~dB}$ respectively. The carrier frequencies are chosen in the predictable region of the filter. For two main reasons:

1. The upper limit of the sPWM generator frequency (approx. $1 \mathrm{MHz}$ )

2. The predictability of the filter response

As the shifting of the carrier frequency in this particular case is limited up to $1 \mathrm{MHz}$, it is not possible to show the effect of shifting in the 'parasitic' region of the filter, which is shown as a gray box in Figure 5.5. Switching frequencies near $100 \mathrm{MHz}$ would be able to demonstrate the possibility to reduce EMI with approximately $20 \mathrm{~dB}$ 'after' integration into the system. The effectiveness of the filter can be rather unpredictable and could be devastating to passing the EMI test, as was shown in [13]. By showing the effect in the predictable region, we demonstrate the possibility to optimize the carrier frequency by means of a 'software' update, i.e. changing the switching frequency after system integration. This could eliminate the need for redesigning a failed system and save time and money.

\subsubsection{The Test Setup}

The setup is shown in Figure 5.2a. The low pass filter at the output is shown as an LC-filter, however the applied output filter has two stages, i.e. it is an L-CL-C filter. It is a COTS filter described in [13], which has a poor performance above approximately $1 \mathrm{MHz}$ as can be seen in Figure 5.5. The equipment used is: 
1. sPWM generator: Digilentic Nexys3 - Xilinx spartan 6

2. Half-bridge: Cree KIT (KIT8020CRD8FF1217P-1)

(a) $\mathrm{SiC}$ based semi-conductors

3. COTS Filter [13]

4. DSO Keysight 3200 with 10:1 voltage probes.

The voltages measured at the input and output of the power-line filter are in the order of several volts. They are acquired at a sampling frequency of $31.25 \mathrm{MHz}$, without any load attached to the system. The DC power supply is set to $7 \mathrm{~V}$, while the dead-time is $150 \mathrm{~ns}$. The dead-time can be set in incremental steps of $10 \mathrm{~ns}$ by the FPGA. Furthermore, the sinusoidal waveform being compared to the triangle carrier wave has a DC-offset of 0.5 and a modulation amplitude of 0.25 . The modulation frequency is set to $2 \mathrm{kHz}$, while the carrier is switched between 24, 50 and $80 \mathrm{kHz}$.

At the input of the filter a unipolar sPWM signal is applied with an amplitude of $7 \mathrm{~V}$ provided by the DC power supply. Low pass filtering the output results in an AC power line signal with a DC offset. To remove the DC offset, a bipolar sPWM at the input of the filter can be used. The resulting measurements are presented in the following section.

\subsubsection{Results}

The measured output voltage waveforms for different carrier frequencies are shown in Figure 5.7, note the phases are not perfectly aligned as the waveforms are recorded with different trigger events. As is to be expected, the resulting waveforms all show the $2 \mathrm{kHz}$ waveform. However in case of the lowest carrier frequency (shown in black), the $2 \mathrm{kHz}$ waveform is modulated with the carrier of $24 \mathrm{kHz}$. To show the effect of the applied filter, the spectral densities of the signals before and after the filter are computed and compared. In Figure 5.8 the input and output spectra are shown in black en red respectively.

It can be seen that for the carrier frequency the peak noise values of the output were reduced with 1,30 and $40 \mathrm{~dB}$ respectively, without reducing or spreading the generated EMI. This might be straightforward as the filter is used in the 'predictable' regime, however here it should be emphasized that the applicability of this technique is to reduce EMI at the integration stage of a system. The filter's response at high frequencies can become unpredictable and dependent on the implementation, due to uncontrollable or unknown parasitics. The reduction of EMI can thus be achieved without compromising the functional behavior of the system. 


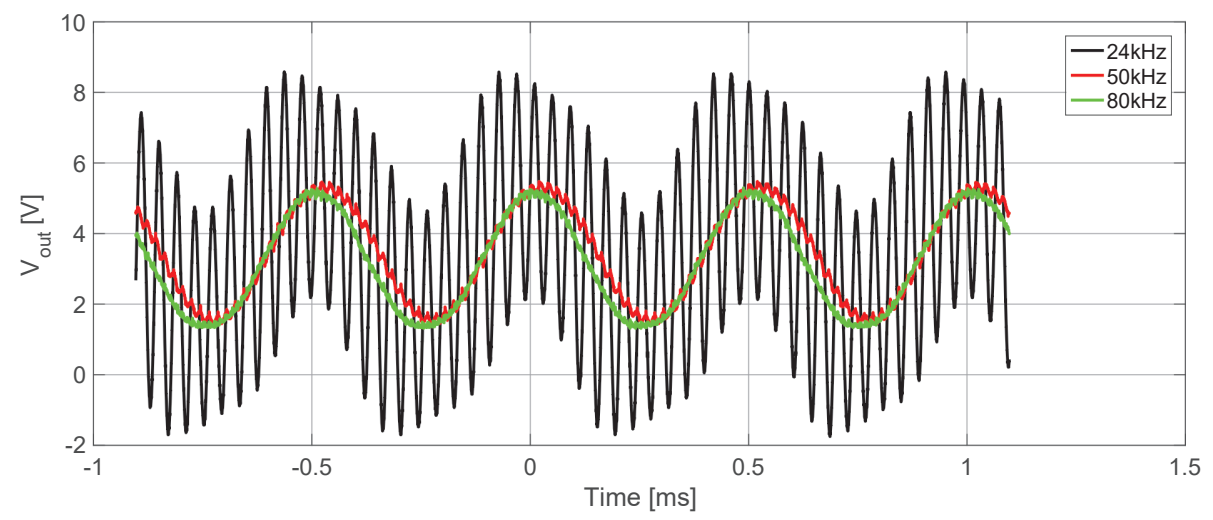

Figure 5.7: Filtered $2 \mathrm{kHz}$ sinewave at 24, 50 and $80 \mathrm{kHz}$ carrier.

\subsection{Radiated EMI Mitigation - Optimal System Us- age}

In Chapter 2 a fast switching GaN DC/AC converter was studied in terms of conducted and radiated emissions. The previous section has shown how choosing the switching frequency carefully can improve conducted EMI, by placing the noise at frequencies where the filter is performing adequately. In the following section a mitigation technique is explored for reducing radiated emissions. The concept is very similar to the conducted mitigation technique, however the filter is now replaced with the system its radiative transfer function. First an explanation of the concept investigated, followed by a short recapitalization is given about the measurements performed in Chapter 2.

\subsubsection{Concept of Radiation Efficiency}

In a modular system like the $\mathrm{M} 2 \mathrm{C}$ and $\mathrm{M} 3 \mathrm{C}$ it is difficult to predict and mitigate the radiated emissions, as they are highly dependent on the final implementation and operation conditions. Nonetheless based on the measurements and/or the theoretical model the spectral components existing in an SM system can be determined. Thus describing the conducted emissions under operating conditions. Combining the knowledge of what components exist in the system and measuring the resulting radiated emissions, one can determine how well these components are transformed into electrical field radiation, i.e. determine the radiation efficiency of the system. Radiation efficiency in this thesis is defined as the ratio between power radiated and power supplied (Equation 5.1). It can be seen as a figure that shows how well something radiates, i.e. a transfer function between conducted and radiated.

Here an effective radiation model is explored, to be used for estimating and predicting radiated noise produced in a sPWM driven power system. In 


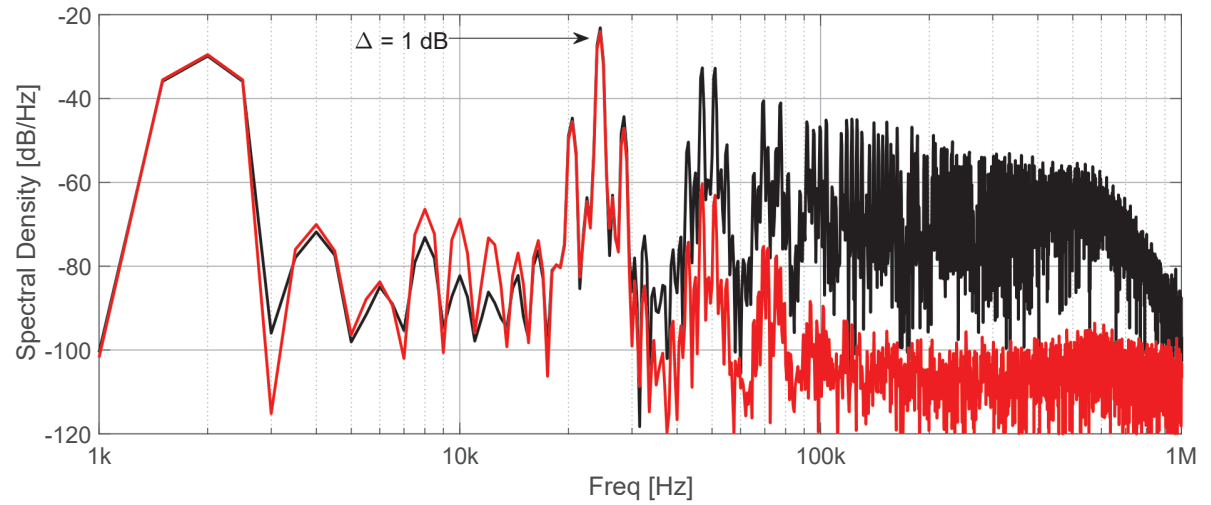

(a) Result of $2 \mathrm{kHz}$ modulation at $24 \mathrm{kHz}$ carrier

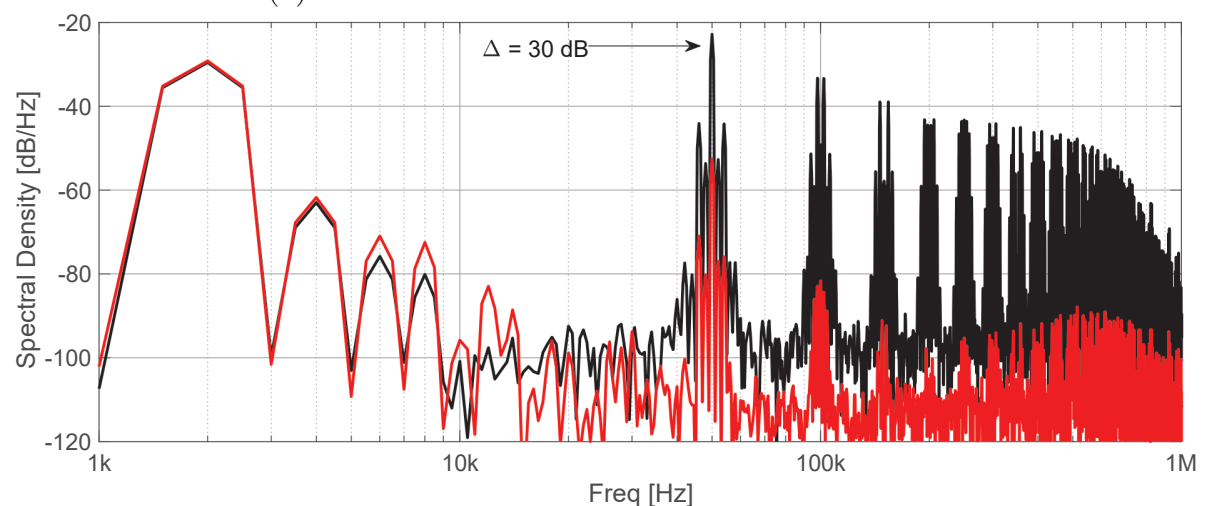

(b) Result of $2 \mathrm{kHz}$ modulation at $50 \mathrm{kHz}$ carrier

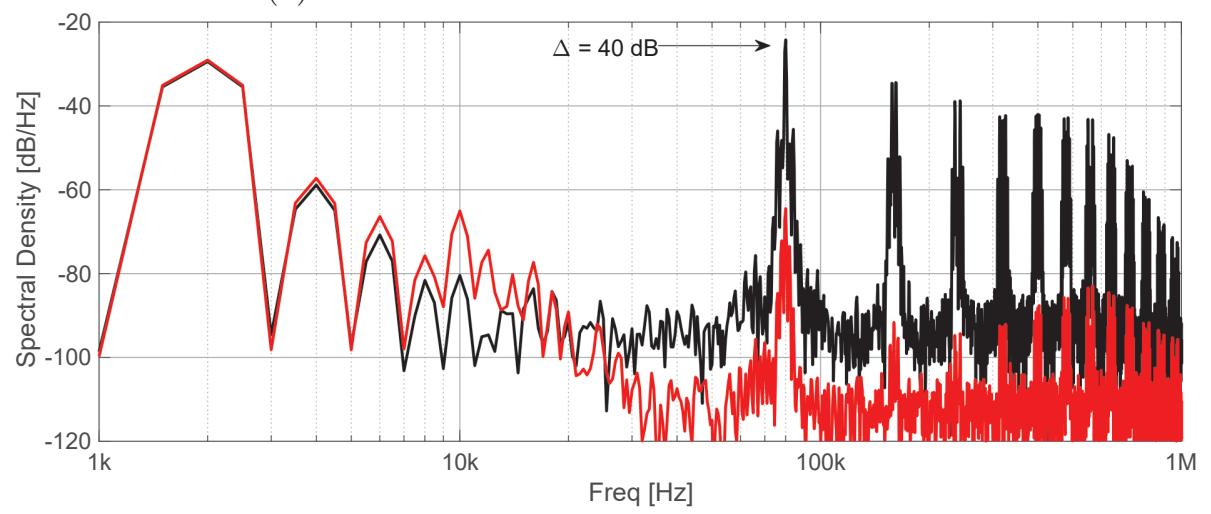

(c) Result of $2 \mathrm{kHz}$ modulation at $80 \mathrm{kHz}$ carrier

Figure 5.8: Power spectral densities of the voltages measured at input(black) and output(red) of the filter. 
the introduction of this chapter it has already been mentioned that it can be used as a mitigation technique but also has potential to be used as a tool for compliance testing. One of the key benefits is that it can estimate emissions of the system under different operational conditions (i.e. different settings for the sPWM generation.) without the need to measure all combinations.

The proposal is based on the transfer function determination in Linear Time-Invariant (LTI) systems. Assuming the entire system to be a LTI black box radiator, which receives a known time signal. In this case the signal is actually created inside the black-box by non-linear switching devices. From LTI system theory it is known that by using the input and output signals one can calculate the transfer function. In Laplace domain this would result in:

$$
H(s)=\frac{Y(s)}{X(s)}
$$

Which in case of antennas translates into radiation efficiency:

$$
\eta(\omega)=\frac{P_{r a d}(\omega)}{P_{\text {sup }}(\omega)}
$$

$P_{\text {sup }}$ is determined through the theoretical analysis of a sPWM signal, which is the output signal of the $\mathrm{DC} / \mathrm{AC}$ converter in this study. A simplified theoretical model for determining $X(s)$ is derived in section 2.1, which is based on parameters available in the generator. $P_{\text {rad }}$ is the measured radiated power, this is done using electrical field measurement through DSP. The power spectra are represented in Figure 5.9a $X(\omega)$ and $Y(\omega)$ respectively. In our measurement setup $Y(\omega)$ is to be derived from the measured time signal $y(t)$.

\subsubsection{Application - DC/AC Converter}

Following the study in [23] the radiated emission of a DC/AC converter is studied. Due to the flexibility in operational modes in the $\mathrm{M} 3 \mathrm{C}$, the amount of measurements required to determine a pass or fail in a EMC test-lab is near infinite. As this requires a lot of time and money it is proposed to estimate the radiation efficiency of the test setup under operating conditions. The switching power system is modeled as a LTI-system that is excited by a known sPWM source, i.e. the switching node voltage waveform. The developed theoretical model in Chapter 2 simplifies the estimation of the sPWM power spectrum. By measuring radiated electric fields and calculating Effective Radiated Power (ERP) one can estimate the system radiation efficiency. Using the radiation efficiency to optimize the EMI spectrum is a novel developed strategy to minimize the total radiated emissions. The generated spectrum can be optimized by removing highly radiative frequency components trough filtering [14] or even harmonic cancellation as was shown in $[22,93]$. 


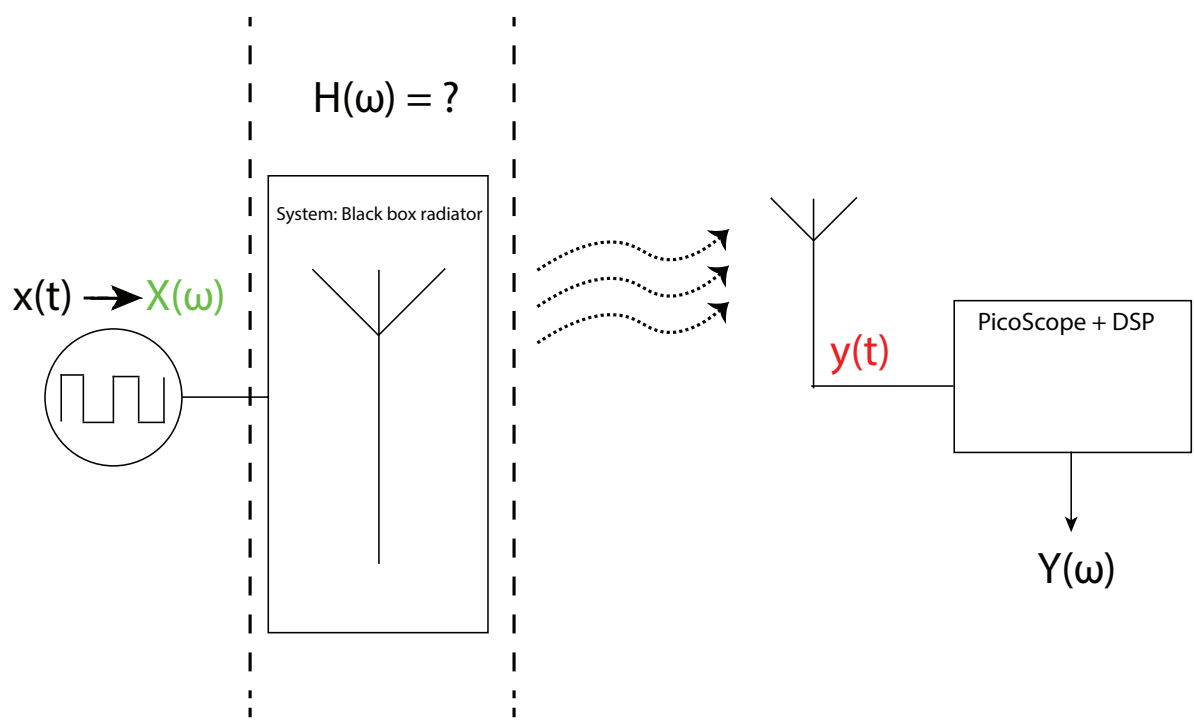

(a) Sketch of the top-level measurement setup with $X(\omega)$ being the theoretical approximated known spectrum and $y(t)$ the measured time signal

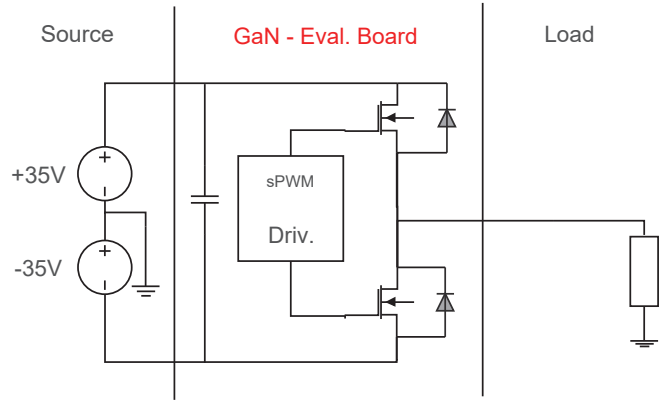

(b) Black Box contents of Figure 5.9a

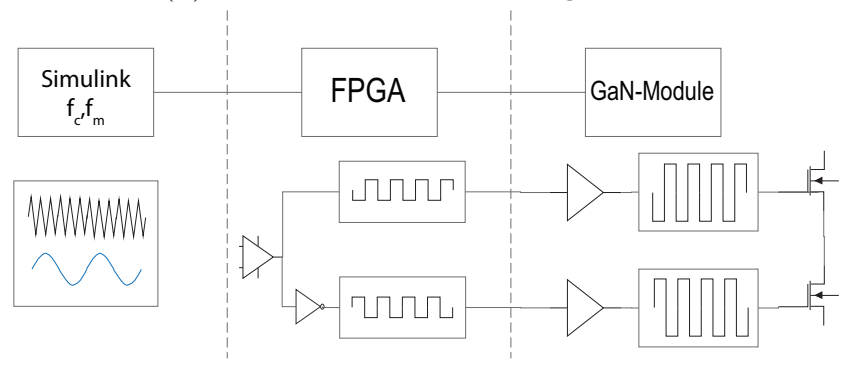

(c) sPWM Driver setup as used in Figure 5.9b

Figure 5.9: Schematic representation of the radiated emission test setup 


\subsection{Radiation Efficiency Determination}

The radiation efficiency is defined as the ratio between radiated power and power supplied to the radiative object or in this case system. In case of an antenna, one could use a VNA with a 'known' and 'unknown' antenna and look at the $S_{21}$ or even use only the $S_{11}$ of unknown antenna. The VNA basically sweeps/chirps a single frequency continuous wave and measures its input/output ports. In case of a system like the $\mathrm{DC} / \mathrm{AC}$ converter, this is difficult to achieve and therefore other methods should be sought. As proposed here, the sPWM generator is able to sweep through different combinations of $f_{c}$ and $f_{m}$ to cover all frequencies as if it was sweeping continues waves.

In Chapter 3 the development of the control system was explained, and shown that the generator has its limits:

1. Carrier Frequency $(10 \mathrm{kHz}-2 \mathrm{MHz})$

2. Modulation Frequency $(0 \mathrm{~Hz}-250 \mathrm{kHz})$

3. AC-amplitude $(0.0-0.5)$

4. DC-offset $(0.0-1.0)$

5. Dead-time ( $0-630 \mathrm{~ns})$

6. Number of levels $(1,2$ or 4$)$

The maximum carrier frequency limits the highest investigatable frequency. In the study performed in [28] the upper limit was set to $100 \mathrm{MHz}$, which results in an approximate wavelength of $3 \mathrm{~m}$. The amplitude of the spectral components are difficult to determine as they are dependent on $f_{c}, f_{m}, m_{i}$, DC-offset and even dead-time [22]. Thus their attenuation is also difficult to determine. By taking the maximum measured component and assume equal transmission over entire bandwidth as determined by Carsons rule at all harmonics, removes the influence $m_{i}$, DC-offset and dead-time by use of overestimation. During the comparison of the theory with measurement results, it is assumed the highest measured peak within a frequency band is radiated anywhere in that rectangular band that was calculated with Equation. 2.10. The radiation efficiency is then determined by averaging the results for different combinations of $f_{c}$ and $f_{m}$, that together will cover almost the entire frequency range of interest.

The measurement setup used is described in Chapter 2 and schematically represented in Figure 2.10. It is the radiated emission test setup that was also used to model the noise source. The results provided there are presented here again, but with the addition of the theoretically determined rectangular bands containing $98 \%$ of the energy at each harmonic of the carrier. This is including the subharmonics present at these frequencies. Figure 5.10a shows this for the first 25 harmonical bands, starting at the switching frequency of approximately $123 \mathrm{kHz}$. In Figure 5.10b a zoomed in version is shown, to explain the extraction method. 


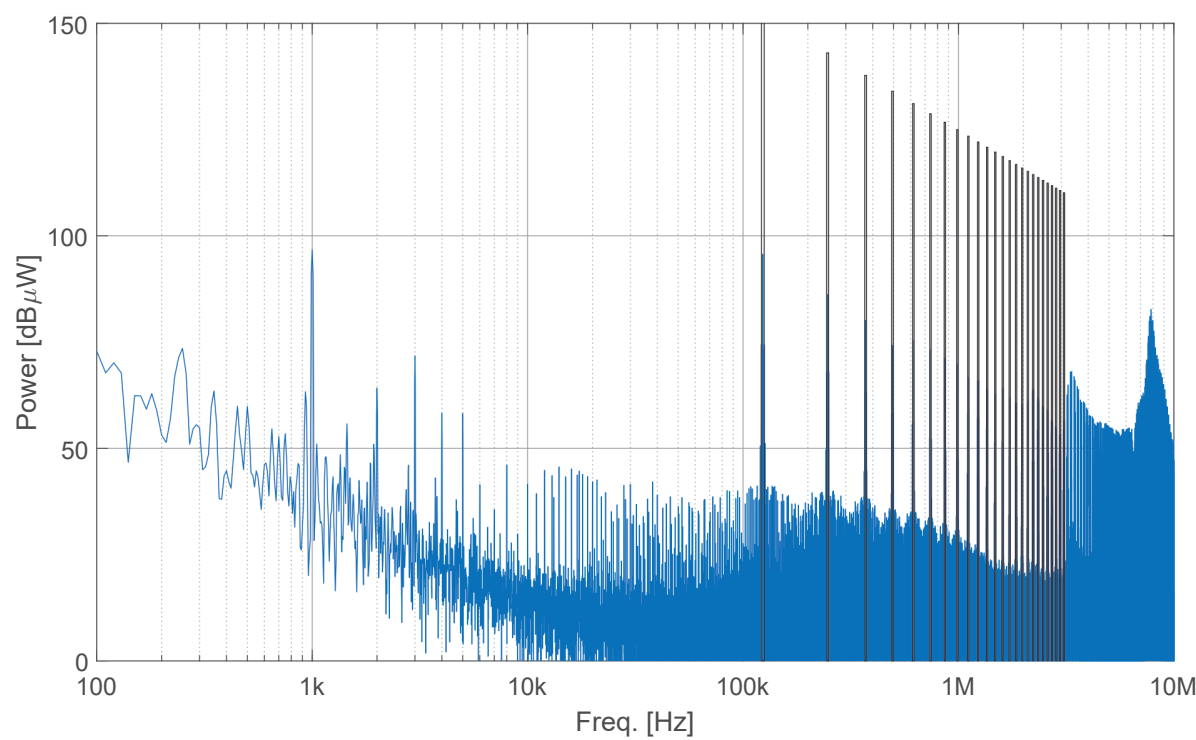

(a) Calculated radiation spectrum with theoretically determined rectangular bands up to the 25th harmonic

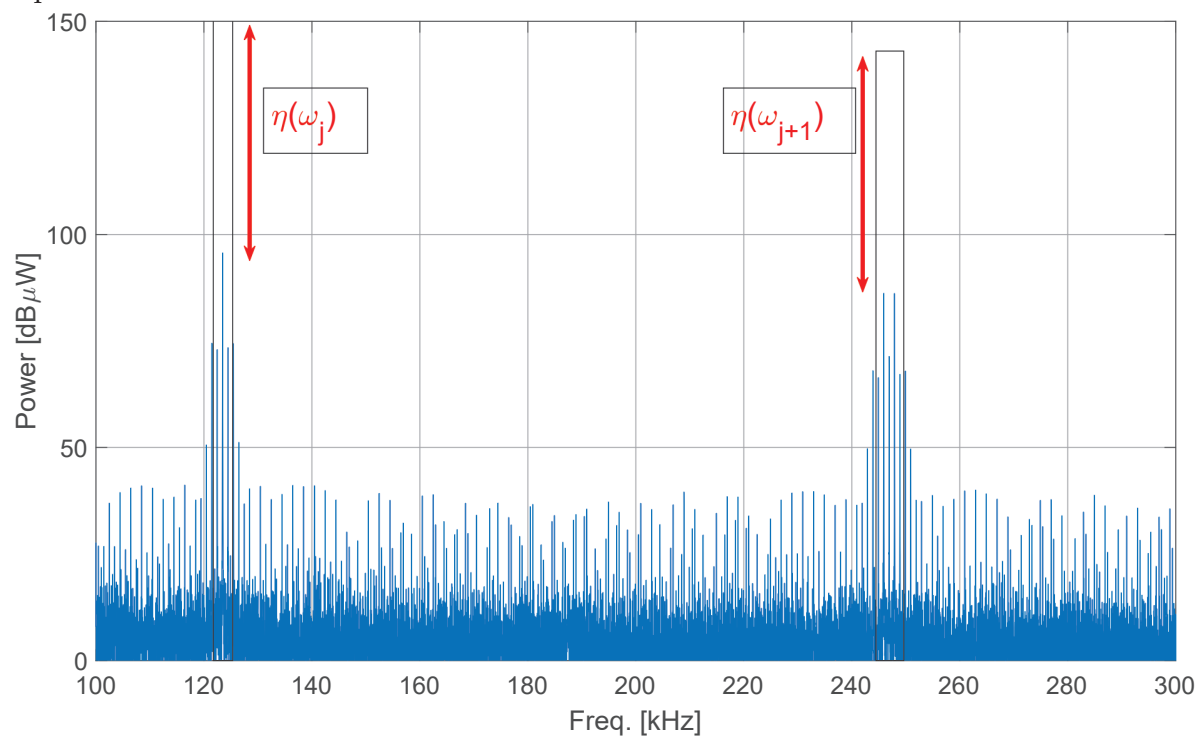

(b) Zoomed in version of plot 5.10a displaying 1st and 2nd harmonic

Figure 5.10: Radiated emission test results 
The theoretical model from Chapter 2 allows the creation of the rectangular black boxes shown in Figure 5.10. As the goal of the model is to approximate all the carrier frequency harmonics and sub-harmonics, it uses the maximum amplitude and bandwidth of each band of harmonics. The maximum amplitude is found by using an approximation for the envelope of the asymptote of the Bessel function (Figure 2.4). i.e. the model does not determine the height of each sub-harmonic, but just calculates the magnitude of the highest (whichever one that is) and uses this as the amplitude of the black box. While the combination with Carsons rule will make sure that the maximum sub-harmonic is located somewhere within this particular harmonical bandwidth. A critical point in the usefulness of this model is that it will create an independence in the final estimation of radiation efficiency on the control parameters $D_{i}, m_{i}$ and $t_{\text {dead }}$.

So the height of the black box shows the theoretically calculated input power at that particular harmonical band, and the deviation is calculated to the maximum received power inside that band. The deviation is directly related to Equation 5.1, as the plot is shown in logarithmic scale the deviation is the ratio between power supplied and received. For further calculations, it is assumed that the found efficiency is constant within this harmonical band.

As Figure 5.10b shows the efficiency results for two harmonical bands. Combining the results of a large set of modulation and carrier frequencies, a single line in Figure 5.12 is created from 100 harmonical bands. Each line shown is created with the same set of frequencies measured, for different modulation indices. i.e. different combinations of $D_{i}$ and $m_{i}$. Figure 5.11 shows the frequency combinations of $f_{m}$ and $f_{c}$ used.

From the results shown in Figure 5.12, it is recommended to avoid generating frequencies of approximately $7.8 \mathrm{MHz}$, as there is clearly a resonance present in the system at that frequency. By shifting the EMI to a more preferred and thus less radiating part of the spectrum, one can reduce emissions by several decibels. Combining the result of Figure 5.12 and the theoretical model, one can now also estimate for non-measured combinations of $f_{m}$ and $f_{c}$ the radiated emissions. Thus a novel method for estimating the radiated EMI spectrum of a half-bridge DC/AC converter in-situ has been developed.

Assuming the generated SPWM is exciting an unknown or undefined passive device/system, that acts as any other LTI system. It was shown in Chapter 2 that in case of a flexible sPWM generator, the amount of possible operational modes is high, which implies a difficult to near impossible task of emission testing. Based on the simplified theoretical model of sPWM presented in Chapter 2, the excitation signal's power spectrum can be estimated. This model has been verified and used for determining the radiation efficiency, i.e. transfer function, of the system under test. In final it was shown that under different operating conditions, i.e. modulation indices and DC-offset combinations, an effective radiation model was found within a $6 \mathrm{~dB}$ margin. It has also been suggested that the generated EMI should be tuned to non-radiative frequencies to lower total emissions. 


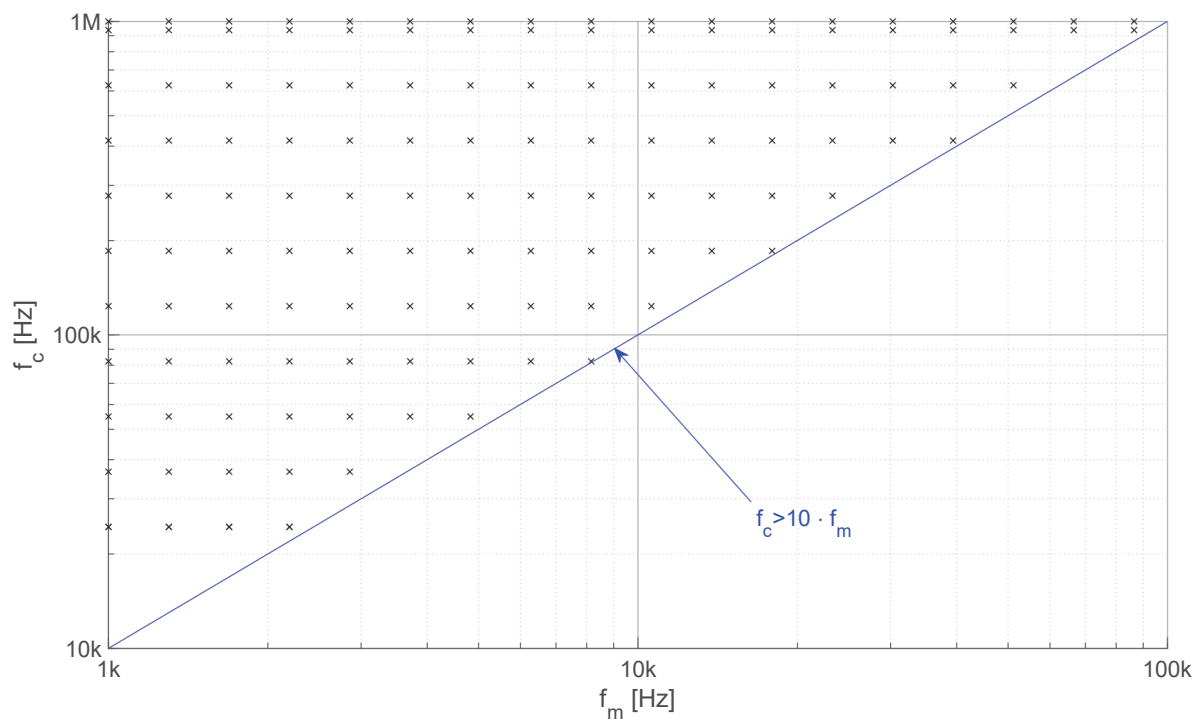

Figure 5.11: 131 measurements have been performed to asses the radiation efficiency for a single line in Figure 5.12

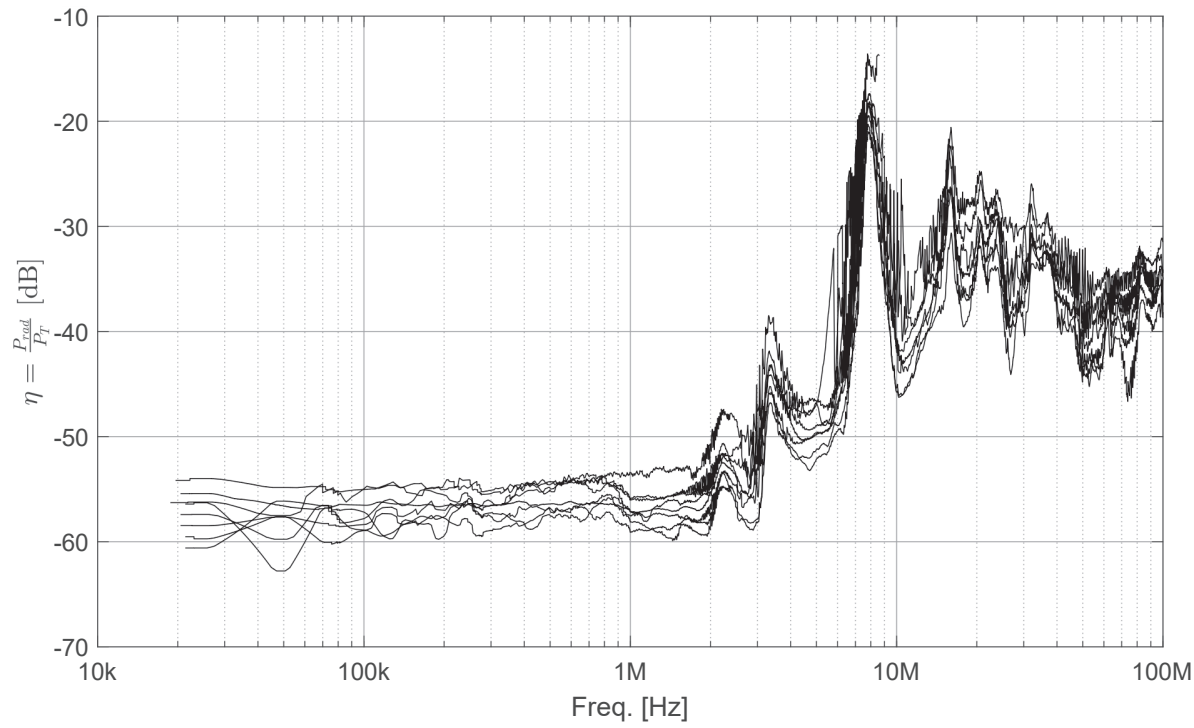

Figure 5.12: Determination of effective radiation model for multiple modulation indexes and DC-offsets represented by each individual line. 


\subsection{Summary}

EMI mitigation is one of the fundamental themes that is used to ensure EMC. Figure 1.6 differentiates different techniques to reduce conducted EMI. In this thesis two paths within this scheme have been explored, mitigation along the propagation path by optimizing filter layout, and mitigation at the source by investigating the switch control scheme. This chapter has combined both to mitigate conducted as well as radiated EMI.

In case of conducted emissions, filtering is applied to reduce EMI. As was shown in amongst others [13], filters can still fail during the integration phase of a system, as is often the case, due to unpredictable parasitic effects. By control of the sPWM generation scheme, one is able to shift the generated noise away from the critical areas of failure. Hence the switch control scheme can be used to reduce EMI by increasing an already implemented filter's effectiveness. This can be done in sPWM control schemes, as the fundamental useful frequency is related to the modulation frequency, while the switching frequency is related independently to the generated EMI.

Following the same principle, the radiated emission can also be reduced. However the challenge here is slightly different, as the parasitic characteristics are challenging to determine in this case, while in case of the filter one might to be able to model them to some extend. Hence for reducing the radiated emissions, a model was derived and a technique developed for estimating the radiation efficiency of a complete system. It assumes the system under test to act as an LTI system, with a known and controllable input source and the output recorded. The input source in this case was the sPWM control scheme, which again has the advantage of controlling the noise generated through changing the switching frequency freely. By sweeping through a set of switching frequencies and measuring the electric fields, one can calculated by post-processing a transfer function of the system. This can then be used to identify an optimal switching frequency with respect to radiated emissions. 


\section{Chapter 6}

\section{Conclusions and Directions for Further Research}

This chapter will shortly recapitulate the key objectives set for the University of Twente in the SMART Grid project. As the chapters have addressed them individually, the chapters are summarized to eventually allow the conclusions to be drawn. At the end of the chapter some directions for further research are given based on some key features of this thesis.

\subsection{Conclusions}

The key objective, as stated in the project proposal plan, for the UT is "to find the best options for reduction of interference, associated with fast switching (and thus highly efficient) semiconductors as applied in the M3C". This resulted in sub-objectives which are related to identifying and possibly mitigating "lowfrequency" conducted EMI, and radiated magnetic and electric fields based on:

1. Full wave models of M3C components and module

2. Behavioral circuit models for network simulation based on the full wave models

3. Experimental validation of the models

4. Optimized filters using novel nano-crystalline materials

5. EMI performance of the prototype

\subsubsection{Chapter 2}

This chapter has been dedicated to describing and evaluating a single halfbridge submodule that can be used in the final multi-level converter. As the 
SM is the basic building block of setup, it is necessary to understand its impact as a noise source. At the beginning of the chapter a basic theoretical model for EMI estimation was given. The model has been compared to measurements, which verifies the EMI generated is directly related to sPWM control signals. Conducted and radiated measurements were performed considering the relative low frequency range between $2 \mathrm{kHz}$ and $150 \mathrm{kHz}$, as this has become a significant research interest. At the moment of writing this thesis, no civil standards exist in this frequency range, but EMI generated here has been identified as the culprit in a rising number of EMC related instances. As the M3C is considered to be a possible cornerstone of the future SMART GRID, compatibility in this frequency range is required even before standards exist. This chapter's contribution thus has been a noise source model can be used to predict the EMI generated.

\subsubsection{Chapter 3}

This chapter has described the development of a sPWM generator for use in a multilevel converter. Its development is aimed at flexibility for research into power systems at much higher frequencies than the conventional approached. It has been shown that the FPGA is able to control 8 half-bridge SM's synchronously, i.e. 16 switches are operated. Carrier frequencies are achieved between $10 \mathrm{kHz}$ and $2 \mathrm{MHz}$, with modulation frequencies between $0 \mathrm{~Hz}$ and $250 \mathrm{kHz}$. Errors introduced by discretization/quantization have been identified and found unacceptable above $5 \%$ deviation. As they are directly related to the carrier frequency, its upper bound has been established as $2 \mathrm{MHz}$ for an FPGA running on a $100 \mathrm{MHz}$ clock. The control logic has been implemented in two $\mathrm{AC} / \mathrm{DC}$ converters measurement setups. It was demonstrated that the modulation and carrier frequency both controllable and the generator capable of being used in multi-level setups.

In the second part of this chapter a numerical analysis was performed to determine the effects of the control parameters $m_{i}, D_{i}$ and phase difference between carrier and modulation wave on the sPWM generation and its spectral content. Apart from the fact that over-modulation generates an enormous amount of noise, the results can be summarized as:

1. Symmetric: when the sinusoidal waveform is symmetrically placed within the triangular waveform, the even numbered subharmonics are suppressed.

2. Asymmetric: when the sinusoidal waveform has a dc-offset compared to the mean of the triangular waveform, the odd numbered subharmonics are in the spectrum.

3. Increasing the amplitude of the sinusoid, the subharmonics will become more significant.

It has also been shown that increasing the amount of stacked sub-modules will effectively increase the switching frequency of system, due to the phase-shifted 
carrier scheme the SM's will switch at different time instances, i.e. increasing the number of SM's has shown to effectively cancel carrier frequencies. Basically shifting the zeroth harmonic of the switching frequency proportional to the number of modules. Intuitively it can also be seen that increasing the number of switchable levels, also reduces the 'to be filtered' noise as it is analogous to quantization noise in which SNR increase with the amount of bits.

Stacking SM's has a large amount of implications, from a power electronic perspective as well as an EMC perspective. The total efficiency of the converter is directly related with the amount of switching operations performed per period of the AC signal, as every turn-on and turn-off event of a switch will dissipate some power. However, lower switching frequencies will increase size and costs of the EMI filtering needed. By stacking modules, the system filters can still be of reduced cost and size, while efficiency can be increased. Semiconductor components also create an upper-level of switchable power often expressed as maximum rated current combined with maximum rated voltage. By stacking either in series or parallel, power systems can divide the load and thus switch high power without the need for specialized development of semiconductor components. In this thesis the trade-offs between the aspects discussed here are neglected and considered to be future work, but in general it is concluded that increasing the amount of sub-modules is beneficial for reducing EMI when using a phase shifted triangular carrier scheme.

\subsubsection{Chapter 4}

Chapter 4 is dedicated to optimizing filter performance through developing more accurate models. First an algorithm has been developed for extracting equivalent circuits from impedance curves based on a Gauss-Newton optimization scheme. It has been applied to individual measurements of components used in CM filter and the measured mutual coupling between these components. The algorithm searches for optimal values for an X-amount of RLC-components in a predefined equivalent circuit. It was shown that it will increase the simulation results accuracy. The development of this algorithm was a necessity for automated back annotation of fields into circuit simulators. The chapter continued with the development of a high frequency full wave model of the CMC. As the CMC is one of the most critical parts of any EMI filter, the development of a 3D full wave model for high frequency $(100 \mathrm{kHz}$ to $1 \mathrm{GHz})$ simulations was required for eventual back annotation of coupling fields.

The third and last part of this chapter describes the technique to include mutual inductive coupling into equivalent circuit simulations and how it can be used to investigate optimal component placement in filters. It has been shown that the filter performance improved with $15 \mathrm{~dB}$ between $5 \mathrm{MHz}$ and $200 \mathrm{MHz}$.

Combining the three techniques shows a method that can automatically back annotate a field effect between components and optimize filter design based on the placement and thus utilizing the field effects, without compromising the speed of the design and the required skill and expertise necessary. This 
can be considered to be the major contribution of this chapter to the thesis. It implies that designing more optimal filters is possible for engineers with limited expert knowledge, which is of great practical value to EMC, power electronic engineers or anyone designing filters.

\subsubsection{Chapter 5}

In Chapter 5 mitigation at the source by investigating the switching control scheme was done. This chapter has combined both mitigation of conducted as well as radiated EMI. Reducing EMI is one of the fundamentals to ensure EMC.

In case of conducted emissions, filtering is applied to reduce EMI. Filters can fail during the integration phase of a system, as is often the case, due to unpredictable parasitic effects. By control of the sPWM generation scheme, one is able to shift the generated noise away from the critical areas of failure. Hence the switch control scheme can be used to reduce EMI by increasing an already implemented filter's effectiveness. This can be done in sPWM control schemes, as the fundamental useful frequency is related to the modulation frequency, while the switching frequency is related independently to the generated EMI.

Following the same principle, the radiated emission can also be reduced. However, inside a complex system it becomes difficult to identify (all) radiative elements. The technique developed in this chapter estimates the radiation efficiency of the entire system with no regard where it originates or what is radiating. It identifies critical frequencies that seem to easily radiate, thus their generation should be avoided to reduce EMI. It assumes the system under test to act as an LTI system, with a known and controllable input source. By sweeping through a set of switching frequencies and measuring the electric fields, one can calculate a transfer function of the system. This can then be used to identify an optimal switching frequency with respect to radiated emissions. It should also hold for magnetic field, however this has not yet been explored and considered to be future work.

\subsection{Directions for Further Research}

The future of the present day electrical grid is still very volatile, in the sense that it is unclear how it will develop. Many new technologies are being developed, that all promise to be the next big breakthrough. The amount of different types of inverter and converters is ever increasing, simultaneously their control schemes and logical processors units are rapidly developing. Regulatory units are unable to cope with the speed of development, which leads to a lack of regulations or even an inability to develop them.

The new technologies do have some similarities that are common in the development of power electronics. Faster switching, leads to lesser losses as the semi-conductor spends less time in a heat dissipating mode. Higher switching frequencies, as this allows for miniaturization of components thus increasing 
power density. Modularization, which reduces costs and allows development of a variety of converters for different applications using the same building blocks. This increases the amount of flexibility required in a converter, that eventually also increases the complexity of the entire system and eventually the grid. Distribution of loads and sources also increase the complexity, while decreasing the possibility to predict EMI.

This thesis has analyzed the M3C as it was developed by TUD in a very general way. The theoretical model developed in Chapter 2 applies to triangular as well as sawtooth wave carriers, while the effects of applying these sawtooth waves were neglected. Also the effects from different types of multi-level carrier schemes have not been investigated. The phase shifted carrier schemes do however show a possibility of canceling switching harmonics as was shown in Chapter 3. Effects of spread-spectrum techniques are considered to be a large topic of interest for the future in these type of converters. Much research in this area exists, however the effect in the novel developed M3C has yet to be investigated and the means for implementation as well. Feedback of measurement data into the control schemes can also still be developed. One of the key benefits of the in-house developed sPWM generator, described in Chapter 3, is that it is developed in Simulink. This allows for rapid development of feedback systems, without an in-depth knowledge of the VHDL programming language which allows the systems control engineers to stay within their area of expertise. Increasing the complexity of sPWM generator should therefore be relatively easy, and is identified as one of the major future research directions as the control signals are directly related to the functional behavior as well as the generated noise.

This thesis has focused on relative low frequency EMI generation and mitigation. The models presented here are often valid up until a few $100 \mathrm{MHz}$. In case of conducted EMI this is considered to be high, however for radiated its just the start. Together with the trend of miniaturization, and increasing switching speeds and frequencies studies addressing frequencies above $100 \mathrm{MHz}$ are also required. Models with increased accuracy with wide applicable bandwidths are necessary, which often increases complexity of the models. This thesis has addressed several key issues, but merely scratched the surface. However the automated back annotation and equivalent circuit model value finding/fitting in Chapter 4 shows promising possibilities to support further research in this area. Increasing complexity of equivalent circuit models inherently increases applicable frequency ranges, however it becomes difficult to ascertain the correct values. Research done in this thesis has shown the applicability in component modeling as well as field modeling. Due to increased interest in power distribution network impedance analyses and modeling, future investigations into speed and accuracy optimization of the algorithm are recommended.

During the research performed in this thesis, several measurement techniques have been explored as well as newly developed. For instance, in Chapter 2 a relation between magnetic field and fast switching DM current has been shown. It exploits the recent developments in low cost base-band digitizers 
with multi-channel capabilities. Further research has already started for developing new measurement techniques for analyzing time-varying impedances, power and energy consumption, fast transients and fields, and even decomposition of EMI. Development of these concepts and thus usage of multi-channel measurement systems are required to cope with the speed of development of power electronics. Availability of cheap, adequate measurement tools allow for better understanding and more carefully design of power systems that from the start are taking care of EMI to allow interoperability.

As is apparent, the complexity of the electromagnetic environment is ever increasing, which implies more expertise is required of the designers to ensure interoperability. This thesis has shown the complexity of a single type of power converter, and contributed in the development of practical analysis tools that can be used in future studies where power electronics meets EMC. The societal necessity for highly skilled engineers with an expertise in conducted EMC can be seen with the approval of two new projects, SCENT and ETOPIA, which will start at the end of the project Smart Grids as described in this thesis.

- SCENT - $\underline{\text { Smart }}$ Cities $\underline{\text { EMC }} \underline{\text { Network and Training }}$

- ETOPIA European Training network $\underline{\mathrm{O}} \underline{\mathrm{PhD}}$ researchers on Innovative EMI analysis and power Applications

Both these project have an emphasis on training young researchers, as there is an urgent need to train new researchers with a closer interaction of electrical power and power electronics with information technology and communications equipment. Interference in the future SMART Grid is one of the most social relevant topics at the moment in EMC. For example, one impactful publication in the recent years have been the erroneous readings in "SMART energy meters", due to the directly related economical impact. 


\section{Chapter 7}

\section{Acknowledgments}

"To boldly go where no one has gone before!"

At the beginning of the $\mathrm{PhD}$, all candidates will be subconsciously telling themselves that they will make a difference in the world of science. Intrinsic motivation is key in any aspect in life, this holds especially for a $\mathrm{PhD}$ track. I think many people have already described the struggle to keep this motivation alive for over four years. Some struggle due to lack of positive feedback, some due to lack in criticisms. Others find the social interactions, or lack off, the most challenging. I think for me it was (or maybe still is), strongly related to the Dunning-Kruger effect. I just hope I'm not still climbing mount stupid. Of course many people have helped me gain the confidence necessary to complete this "mission". Here I would like to take the opportunity to thank them.

I would like to start with thanking my promoter Frank for giving me the opportunity to do a PhD. You have transferred to me so much more than purely scientific knowledge. I'm still awed with your ability to motivate people to give their best, and push beyond their own perceived boundaries, even when you sometimes might be pushing your own boundaries a bit too far. Thank you for sending me across the globe to talk with peers in cities like Washington, Shenzen, Singapore, Angers, Dresden, Wroclaw and the most important one Stellenbosch. Aside from a good working ethic, you have also shown me not to forget about the more important things in life. "Don't worry, be happy".

I would also like to thank Mark, whom during almost the entire period of my $\mathrm{PhD}$, was the chairman of the Telecommunications group. Without you, I would have never even considered doing a PhD. By getting me an internship at one of most prestigious universities in the world, you actually showed me that I could do more than I thought possible. During this period you also suggested me to apply for a PhD position at the group, however it would be under the supervision of Frank, meaning, to drift away from astronomy. Which in hindsight was a blessing in disguise.

Lilian, without you, I would have never been able to coop with the many different aspects of doing $\mathrm{PhD}$. From stacks of paperwork related to traveling 
(insurance, visas, registration etc.), to the reservation of meeting rooms for bachelor/master assignment presentations and rehearsals, and the submission of assessment forms of the many students that passed through this group in last 4 years. Also thank you for dealing with my frustrations during this period, and being our personal psychologist. However, in my humble opinion, the most important aspect of you being in this group is the care taking of anyone who needs your help in whatever way. A true patroness!

During my four years as a $\mathrm{PhD}$ researcher, there has been a lot of change with a large variation in group composition and also group chemistry. Nevertheless, one constant factor throughout this journey has been the "Oliebollen" group. I could always count on you guys for procrastination and fried chicken. The example you have set for me was often like a double-edged sword. Dr. Robert and dr. Stefan, during several conferences you have shown me the importance of networking after the symposium-program ended, deep into the wee hours of the next morning. The most impressive feature of you was your obedience to the dutch (unofficial) proverb: "'s avond een vent, 's ochtends een vent". Of course it has not all been fun and games, you also have shown me how to critically look at my work and push myself. Thank you for helping me to become confident in the work done and how to present it in a public speaking manner.

Dr. Ibrahim and dr. Alex, even though you were at the dark side of the Telecommunications group, I enjoyed spending time with you guys. Alex, thank you for allowing me to set up shop at the best spot in the $\mathrm{PhD}$ room. I've passed on your legacy of reminding the new occupant to not be a feline character when things get though. Ibi, I think you have been my longest office mate during this period. First in the general $\mathrm{PhD}$ office, but later also in our private office. Thank you for being my wikipedia on mathematics, you were faster and more accessible than google's search engine.

Here I would like to take the opportunity to thank the students that provided a contribution to this thesis: Rowan, Chris, Eelco and Wei. Thank you for providing me with the terabytes of data from several measurement campaigns that took countless of hours to acquire. Continuing with thanking contributors, I would also like thank Anne and Jesper. Anne, I've spent many hours studying and reading your thesis. The complexity of the CMC and your solutions how to model this component truly inspired me. I enjoyed the discussions we had and hope to continue to have with respect to this work. Jesper, buddy, as a mathematician you have helped me to reduce complex, higher order problems to linear and thus more manageable calculations. We have visited all EMC Europe symposia together, starting in Dresden (2015) to the latest in Barcelona (2019). Thank you for all the laughter, which I hope will continue for many years. If not, we'll always have Angers and Indonesia.

Apart from thanking the contributing factors of this thesis, I would like to continue giving shout-outs to my colleagues of whom I had the pleasure to socialize with in many different settings. The more experienced people like Frits, Dwi, Arjan and Cees have shown me how to behave (or not behave) in formal occasions and act professionally in a working environment. As a 
counterbalance, I would also like to thank the twins Roelof and Robert for the informal and little bit less professional environment involving many threshold lowering substances, or in Cees his words: "Tarwesmoothies". Thank you for making the lab a fun place to be and showing me the rope as fresh master student, for me it was the start of less aversion towards the educational system.

The latest addition to our EMC family, at least at the final stage of my $\mathrm{PhD}$, are Tom and Bas. Without you, I would have probably been working in industry now. So thank you for joining, and rejuvenating the group and creating a pleasant atmosphere. I look forward to bossing you around for the next 2 years.

Although this thesis has been a professional highlight in my life, it could not have been achieved without the personal aspect. As this part was developed in the earlier stages of my life, where skill in the English language was not yet required nor developed. Hence I will now continue in Dutch, my native tongue.

Beste Mam en Pap, ik neem niet vaak genoeg de tijd om jullie te bedanken voor alles wat jullie hebben gedaan en nog steeds doen voor me. Ik hoop dat jullie desondanks wel beseffen dat het uit de grond van mijn hart komt als ik zeg, bedankt voor alle liefde en kansen die jullie me hebben gegeven. Uiteraard wil ik ook mijn familie hierin bedanken. Als bijna de jongste van het stel ooms, tantes, neven en nichten heb ik altijd met veel bewondering om mij heen gekeken.

Maar naast familie hebben vrienden, maatjes, kameraden altijd een belangrijke rol gespeeld. Thijs, Sam, Thomas, Elias, Enes en Mathijs, bedankt dat jullie altijd klaarstaan voor me, ondanks de vele abracadabra verhalen van technische oorsprong. Ook bedankt voor vele keren dat ik het internet mocht komen fixen of apparaten aan mocht komen sluiten. Toch makkelijk zo'n persoonlijke monteur, "want je doet toch iets met kabeltjes en elektriciteit!?". Het ging in ieder geval, en gaat nog steeds, altijd gepaard met een glimlach. De jongens die ik ook nog wil bedanken zijn gelukkig wat technischer aangelegd. Pim en Tjarco, bedankt voor de mooie studententijd, waar deze $\mathrm{PhD}$ toch stiekem een beetje bij hoort. Uiteraard zijn er nog velen die mijn leven hebben beïnvloed wie ik hier niet persoonlijk benoem maar toch zou willen bedanken. Er is vast een beroemde uitspraak over hoe men een optelsom is van de mensen in je directe omgeving, dank allen voor de positieve energie die jullie allen uitstralen. Dan rest mij nog één speciaal iemand die ik graag wil bedanken, mijn toekomstige vrouw, Kyra. Hetgeen waar sommige eerder genoemden maar af en toe mee te maken hebben, ga jij toezeggen de rest van je leven mee opgescheept te willen zijn. Daar kan ik niets anders dan bewondering voor hebben. Mijn dank is dan ook groot dat je naast me hebt gestaan tijdens dit 4 jarige traject en dat je me wilt bijstaan in jaren die nog gaan volgen. Ik heb je dan ook heel hard nodig. 


\section{Chapter 8}

\section{Biography}

Niek Moonen was born in Veghel, the Netherlands, in May 1990. He graduated from the bachelor Advanced Technology in 2012 and from the master Electrical Engineering in 2014. Both educations were performed at the University of Twente. His masters graduation project was an extension of the internship conducted at Massachusetts Institute of Technology (M.I.T.). The goal of the project was to develop a distributed correlator for space based applications. The correlator was programmed via Simulink on Field Programmable Gate Arrays (FPGAs). Since January 2015 he has been working as a Ph.D. candi-

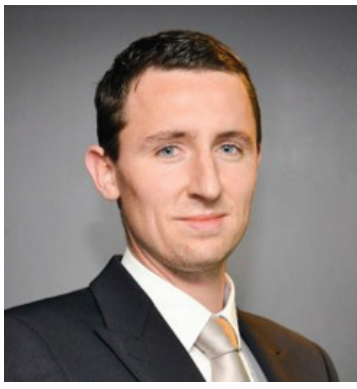
date in ElectroMagnetic Compatibility (EMC) at the Telecommunication Engineering group at the University of Twente, Enschede. Niek has been involved in the project "Smart Grid", in which he was responsible for identifying and possibly mitigating electromagnetic interference associated with fast switching semiconductors applied in a multi-function multi-level modular converter. In January 2019 he started as a Researcher at the same group, and is involved in the projects "SCENT", "ETOPIA" and "Smart Meters" as a supervisor. He is also a member of the IEEE EMC Society, reviewer for IEEE Transactions on EMC and the young professional member of Technical Committee 7 - Low Frequency EMC. 
536329-L-bw-Moonen

Processed on: 3-10-2019 


\section{References}

[1] Administration U.S. Energy Information, "https://www.eia.gov/electricity/data/browser/," 2018.

[2] H. Farhangi, "The path of the smart grid," IEEE Power and Energy Magazine, vol. 8, no. 1, pp. 18-28, 2010.

[3] M. Gagic, T. Hailu, and J. A. Ferreira, "Multifunctional Modular Multilevel Converter-based Systems Bottom-Up Approach to System Design," in 2016 IEEE 8th International Power Electronics and Motion Control Conference, IPEMC-ECCE Asia 2016. Hefei: IEEE, 2016, pp. 944-951.

[4] F. Kienle and C. de Schryver, " $100 \%$ Green Computing At The Wrong Location?" IEC-IEEE CHALLANGE: 2012-03-1128, no. October 2012, 2012 .

[5] J. A. Ferreira, "The multilevel modular DC converter," IEEE Transactions on Power Electronics, vol. 28, no. 10, pp. 4460-4465, 2013.

[6] —_ "Nestled secondary power loops in multilevel modular converters," 2014 IEEE 15th Workshop on Control and Modeling for Power Electronics, COMPEL 2014, pp. 1-9, 2014.

[7] A. Mora, M. Urrutia, R. Cardenas, A. Angulo, M. Espinoza, M. Diaz, and P. Lezana, "Model Predictive Control Based Capacitor Voltage Balancing Strategies for Modular Multilevel Converters," IEEE Transactions on Industrial Electronics, vol. 0046, no. ii, pp. 1-1, 2018.

[8] B. Fan, K. Wang, P. Wheeler, C. Gu, and Y. Li, "An Optimal Full Frequency Control Strategy for the Modular Multilevel Matrix Converter Based on Predictive Control," IEEE Transactions on Power Electronics, vol. 33, no. 8, pp. 1-1, 2017.

[9] S. Debnath, J. Qin, B. Bahrani, M. Saeedifard, and P. Barbosa, "Operation, Control, and Applications of the Modular Multilevel Converter: A Review," IEEE Transactions on Power Electronics, vol. PP, no. 99, pp. 1-1, 2014. 
[10] K. Huang and J. A. Ferreira, "Two Operational Modes of the Modular Multilevel DC Converter," in Power Electronics and ECCE Asia (ICPEECCE Asia), 2015 9th International Conference on, 2015, pp. 1347-1354.

[11] S.-P. Weber, E. Hoene, S. Guttowski, W. John, and H. Reichl, "Predicting Parasitics and Inductive Coupling in EMI-Filters," in Annual IEEE Conference Applied Power Electronics Conference and Exposition (APEC). IEEE, 2006, pp. 1157-1160.

[12] S. Wessling and S. Dickmann, "Predicting the conducted emissions of switched-mode power converters including component and printed circuit board parasitics," in IEEE International Symposium on Electromagnetic Compatibility. Dresden: IEEE, 2015, pp. 1406-1411.

[13] N. Moonen, F. Buesink, and F. Leferink, "Unexpected poor performance of presumed high-quality power line filter, and how it improved," in 2015 IEEE International Symposium on Electromagnetic Compatibility (EMC). Dresden: IEEE, aug 2015, pp. 382-385.

[14] —, "Current Barriers to Confine High Frequency Common Mode Currents," in International Symposium on Electromagnetic Compatibility (EMC EUROPE). Wroclaw: IEEE, 2016, pp. 586-589.

[15] K. Mainali and R. Oruganti, "Conducted EMI mitigation techniques for switch-mode power converters: A survey," IEEE Transactions on Power Electronics, vol. 25, no. 9, pp. 2344-2356, 2010.

[16] Z. Quan and Y. W. Li, "Impact of PWM Schemes on the Common Mode Voltage of Interleaved Three-Phase Two-Level Voltage Source Converters," IEEE Transactions on Industrial Electronics, vol. 66, no. 2, pp. 852-864, 2019.

[17] H. P. Park and J. H. Jung, "PWM and PFM Hybrid Control Method for LLC Resonant Converters in High Switching Frequency Operation," IEEE Transactions on Industrial Electronics, vol. 64, no. 1, pp. 253-263, 2017.

[18] K. K. Tse, H. S. H. Chung, S. Y. R. Hui, and H. C. So, "A comparative study of carrier-frequency modulation techniques for conducted EMI suppression in PWM converters," IEEE Transactions on Industrial Electronics, vol. 49, no. 3, pp. 618-627, 2002.

[19] S. R. Bowes and D. Holliday, "Optimal regular-sampled PWM inverter control techniques," IEEE Transactions on Industrial Electronics, vol. 54, no. 3, pp. 1547-1559, 2007.

[20] W. R. Liou, H. M. Villaruza, M. L. Yeh, and P. Roblin, "A digitally controlled low-EMI SPWM generation method for inverter applications," IEEE Transactions on Industrial Informatics, vol. 10, no. 1, pp. 73-83, 2014. 
[21] M. L. Yeh, W. R. Liou, H. P. Hsieh, and Y. J. Lin, "An electromagnetic interference (EMI) reduced high-efficiency switching power amplifier," IEEE Transactions on Power Electronics, vol. 25, no. 3, pp. 710-718, 2010.

[22] N. Moonen, M. Gagic, F. Buesink, J. A. Ferreira, and F. Leferink, "Harmonic cancellation in a novel multilevel converter topology for the future smart grid," in 2017 IEEE International Symposium on Electromagnetic Compatibility \& Signal/Power Integrity (EMCSI). Washington: IEEE, aug 2017, pp. 602-607.

[23] N. Moonen, F. Buesink, and F. Leferink, "EMI reduction in sPWM Driven $\mathrm{SiC}$ converter based on carrier frequency selection," in 2017 International Symposium on Electromagnetic Compatibility - EMC EUROPE. Angers: IEEE, sep 2017, pp. 1-5.

[24] G. Asmanis, A. Asmanis, and D. Stepins, "Mutual couplings in three phase T-type EMI filters," in International Symposium on Electromagnetic Compatibility - EMC EUROPE, EMC Europe. Roma: IEEE, 2012, pp. $1-6$.

[25] S. Wang, F. C. Lee, and J. D. van Wyk, "Inductor winding capacitance cancellation using mutual capacitance concept for noise reduction application," IEEE Transactions on Electromagnetic Compatibility, vol. 48, no. 2, pp. 311-318, 2006.

[26] S. Wang, F. C. Lee, W. G. Odendaal, and J. D. V. Wyk, "Improvement of EMI Filter Performance with Parasitic Coupling Cancellation," in 2005 IEEE 36th Power Electronics Specialists Conference, Recife, Brazil, 2005, pp. $1780-1786$.

[27] F. Buesink, B. V. Leersum, and F. Leferink, "Reliable systems design using current boundaries," IEEE Electromagnetic Compatibility Magazine, vol. 6 , no. 1 , pp. 56-61, 2017.

[28] C. Van Diemen, N. Moonen, and F. Leferink, "Estimation of Radiation Efficiency of GaN Half-Bridge Based Submodule System for Radiated EMI Prediction," in 2018 International Symposium on Electromagnetic Compatibility (EMC EUROPE), First Runner-Up Best Student Paper Award. Amsterdam: IEEE, aug 2018, pp. 102-107.

[29] G. S. Konstantinou and V. G. Agelidis, "Performance evaluation of halfbridge cascaded multilevel converters operated with multicarrier sinusoidal PWM techniques," in 2009 4th IEEE Conference on Industrial Electronics and Applications, ICIEA 2009, 2009, pp. 3399-3404.

[30] F. Vasca and L. Iannelli, Dynamics and control of switched electronic systems: advanced perspectives for modeling, simulation and control of power converters. Springer, 2012. 
[31] M. Abramowitz and I. A. Stegun, Handbook of mathematical functions: with formulas, graphs, and mathematical tables. Courier Corporation, 1964, vol. 55 .

[32] T. Haykin, Simon S and Moher, Michael and Song, An introduction to analog and digital communications. New York: Wiley New York, 1989.

[33] I. Setiawan, N. Moonen, F. Buesink, and F. Leferink, "Efficient magnetic field measurements," in 2017 International Symposium on Electromagnetic Compatibility - EMC EUROPE. IEEE, sep 2017, pp. 1-4.

[34] T. Hartman, N. Moonen, and F. Leferink, "Direct Sampling in Multichannel Synchronous TDEMI Measurements," in 2018 IEEE 4th Global Electromagnetic Compatibility Conference (GEMCCON). Stellenbosch: IEEE, nov 2018, pp. 1-5.

[35] T. Hartman, N. Moonen, B. ten Have, and F. Leferink, "Fast Magnetic Emission Tests for Continuous Measurements Around an Equipment Under Test," in ESA Workshop on Aerospace EMC. Budapest: IEEE, 2019.

[36] N. Moonen, "Harmonic cancellation in a Multi Level converter," pp. 1-2, 2017.

[37] M. W. Naouar, E. Monmasson, A. A. Naassani, I. Slama-Belkhodja, and N. Patin, "FPGA-based current controllers for AC machine drives - A review," IEEE Transactions on Industrial Electronics, vol. 54, no. 4, pp. 1907-1925, 2007.

[38] E. Monmasson and M. Cirstea, "FPGA Design Methodology for Industrial Control Systems - A Review," IEEE Transactions on Industrial Electronics, vol. 54, no. 4, pp. 1824-1842, 2007.

[39] N. Sahu, N. D. Londhe, and G. B. Kshirsagar, "FPGA applications in inverter and converter circuits: A review on technology, benefits and challenges," Proceedings of 2017 International Conference on Innovations in Information, Embedded and Communication Systems, ICIIECS 2017, pp. $1-5,2018$.

[40] T. Mishima and E. Morita, "High-Frequency Bridgeless Rectifier-based ZVS Multi-Resonant Converter for Inductive Power Transfer Featuring High-voltage GaN-HFET," IEEE Transactions on Industrial Electronics, vol. 0046, no. c, pp. 9155-9164, 2017.

[41] M. Lakka, E. Koutroulis, and A. Dollas, "Development of an FPGA-based SPWM generator for high switching frequency DC/AC inverters," IEEE Transactions on Power Electronics, vol. 29, no. 1, pp. 356-365, 2014.

[42] J. Liu, W. Yao, and Z. Lu, "Design and Implementation of DSP based High-Frequency SPWM Generator," in 2016 IEEE 8th International Power 
Electronics and Motion Control Conference (IPEMC-ECCE Asia). Hefei: IEEE, 2016.

[43] E. Noorsal, I. R. Ibrahim, A. F. Rahim, and Z. I. Rizman, "Multilevel inverter switching controller using a field programmable gate array (FPGA)," Journal of Fundamental and Applied Sciences, vol. 6S, no. 9, pp. 684-709, 2017.

[44] N. M. Salgado-Herrera, J. A. Medina-Ríos, R. Tapia-Sánchez, O. AnayaLara, and J. R. Rodríguez-Rodríguez, "DSPWM multilevel technique of 27 - levels based on FPGA for the cascaded DC / AC power converter operation," International Transactions on Electrical Energy Systems, no. August 2017, pp. 1-15, 2018.

[45] J. Liu, K. W. E. Cheng, and J. Zeng, "A unified phase-shift modulation for optimized synchronization of parallel resonant inverters in high frequency power system," IEEE Transactions on Industrial Electronics, vol. 61, no. 7, pp. 3232-3247, 2014.

[46] S. Sau and B. G. Fernandes, "Modular Multilevel Converter Based Variable Speed Drive with Reduced Capacitor Ripple Voltage," IEEE Transactions on Industrial Electronics, vol. PP, no. c, pp. 1-1, 2018.

[47] V. N. S. R. Jakka, A. Shukla, and S. V. Kulkarni, "Flexible Power Electronic Converters for Producing AC Superimposed DC (ACsDC) Voltages," IEEE Transactions on Industrial Electronics, vol. 65, no. 4, pp. 3145-3156, 2018.

[48] H. Peng, J. Sabate, K. A. Wall, and J. S. Glaser, "GaN-Based HighFrequency High-Energy Delivery Transformer Push-Pull Inverter for Ultrasound Pulsing Application," IEEE Transactions on Power Electronics, vol. 33, no. 8, pp. 6794-6806, 2018.

[49] M. Gagic and J. A. Ferreira, "Multifrequency Electrical Systems: From Power Transmission to Power Electronic Converters," in Power Electronics Conference (SPEC), 2017 IEEE Southern. Puerto Varas, Chile: IEEE, 2017, pp. 1-7.

[50] H. Calleja and J. Pacheco, "Frequency Spectra of Pulse-Density Modulated Waveforms," in Power Electronics Congress, 2000. CIEP 2000. VII IEEE International. IEEE, 2000, pp. 223-228.

[51] P. Zumel, O. García, J. A. Oliver, and J. A. Cobos, "Differential-mode EMI reduction in a multiphase DCM flyback converter," IEEE Transactions on Power Electronics, vol. 24, no. 8, pp. 2013-2020, 2009.

[52] M. S. A. Dahidah, G. Konstantinou, and V. G. Agelidis, "A Review of Multilevel Selective Harmonic Elimination PWM: Formulations, Solving Algorithms, Implementation and Applications," IEEE Transactions on Power Electronics, vol. 30, no. 8, pp. 4091-4106, 2015. 
[53] N. Moonen, J. L. Rotgerink, and F. Leferink, "Automated equivalent circuit extraction of impedance curves using a Gauss-Newton algorithm," in 2018 IEEE International Symposium on Electromagnetic Compatibility and 2018 IEEE Asia-Pacific Symposium on Electromagnetic Compatibility (EMC/APEMC). Singapore: IEEE, may 2018, pp. 1103-1108.

[54] N. Moonen, A. Roc'h, and F. Leferink, "Influence of mutual coupling on parasitic capacitance in common mode chokes," in 2018 IEEE International Symposium on Electromagnetic Compatibility and 2018 IEEE Asia-Pacific Symposium on Electromagnetic Compatibility (EMC/APEMC). Singapore: IEEE, may 2018, pp. 1305-1309.

[55] N. Moonen, R. Vogt-Ardatjew, A. Roc'h, and F. Leferink, "3-D Full-Wave High Frequency Common Mode Choke Modeling," IEEE Transactions on Electromagnetic Compatibility, no. (Early Access), pp. 1-8, 2019.

[56] N. Moonen, F. Buesink, and F. Leferink, "Optimizing capacitor placement in EMI-filter using back annotation of 3D field coupling parameters in circuit models," in 2016 International Symposium on Electromagnetic Compatibility - EMC EUROPE. Wroclaw: IEEE, sep 2016, pp. 576-580.

[57] C. R. Paul, Introduction to Electromagnetic Compatibility (Wiley Series in Microwave and Optical Engineering). Wiley-Interscience, 2006.

[58] N. Moonen, F. Buesink, and F. Leferink, "Enhanced circuit simulation using mutual coupling parameters obtained via 3D field extraction," in 2016 Asia-Pacific International Symposium on Electromagnetic Compatibility (APEMC). Shenzhen, China: IEEE, may 2016, pp. 181-183.

[59] G. Asmanis, "Measurement and Modeling of EMI Filters," Ph.D. dissertation, Riga Technical University, 2014.

[60] T. D. Oliveira, J.-M. Guichon, J.-L. Schanen, and L. Gerbaud, "PEECmodels for EMC filter layout optimization," 2010 6th International Conference on Integrated Power Electronics Systems, pp. 1-6, 2010.

[61] I. Kovacevic and T. Friedli, "3-D electromagnetic modeling of parasitics and mutual coupling in EMI filters," IEEE Transactions on Power Electronics, vol. 29, no. 1, pp. 135-149, 2014.

[62] G. Asmanis, D. Stepins, A. Asmanis, and L. Ribickis, "Capacitors mutual inductance modeling and reduction," in International Symposium on Electromagnetic Compatibility, EMC Europe. Gothenburg, Sweden: IEEE, 2014, pp. 1176-1181.

[63] M. Illia and G. Griepentrog, "Finite Element Method Based Electromagnetic Modeling of Three-Phase EMI Filters," in Power Electronics Conference (SPEC), IEEE Annual Southern. IEEE, 2016, pp. 1-6. 
[64] J. H. G. J. Lansink Rotgerink and H. Schippers, "Crosstalk modelling of unshielded wire pairs," in IEEE International Symposium on Electromagnetic Compatibility, vol. 0, no. 1, Gothenburg, Sweden, 2014, pp. 641-646.

[65] —_ "Generic prediction of crosstalk between shielded wires," in IEEE International Symposium on Electromagnetic Compatibility, vol. 2015-Septm. Dresden: IEEE, 2015, pp. 616-621.

[66] J. H. G. J. Lansink Rotgerink, F. Happ, and J. J. P. van Es, "Crosstalk between wire pairs above a composite ground plane," in IEEE International Symposium on Electromagnetic Compatibility, vol. 2016-Novem, Wroclaw, 2016, pp. 89-93.

[67] S. Wang, F. C. Lee, and W. G. Odendaal, "Characterization and parasitic extraction of EMI filters using scattering parameters," IEEE Transactions on Power Electronics, vol. 20, no. 2, pp. 502-510, 2005.

[68] L. Asmanis, G. and Stepins, D. and Asmanis, A. and Ribickis, G. Asmanis, D. Stepins, A. Asmanis, and L. Ribickis, "Mutual Couplings between EMI Filter Components," in IEEE International Symposium on Electromagnetic Compatibility. Dresden: IEEE, 2015, pp. 908-913.

[69] A. Barchanski, C. S. T. Computer, and S. Technology, "Linking Circuit Simulation with Full-Wave Solver for Board-Level EMC Design," IEEE Electromagnetic Compatibility Magazine, vol. 4, no. 3, pp. 52-58, 2015.

[70] M. Kovacic, Z. Hanic, S. Stipetic, S. Krishnamurthy, and D. Zarko, "Analytical wideband model of a common-mode choke," IEEE Transactions on Power Electronics, vol. 27, no. 7, pp. 3173-3185, 2012.

[71] A. Roc'h, "Behavioural models for common mode EMI filters," Ph.D. dissertation, University of Twente, 2012.

[72] A. Roc'h and F. Leferink, "Contributing factors in the final performance of a common mode choke," in IEEE International Symposium on Electromagnetic Compatibility. Dresden: IEEE, 2015, pp. 120-123.

[73] L. Dehong and J. Jianguo, "High frequency model of common mode inductor for EMI analysis based on measurements," in 3rd International Symposium on Electromagnetic Compatibility, Beijing, China, 2002, pp. 462-465.

[74] M. L. Heldwein, L. Dalessandro, and J. W. Kolar, "The three-phase common-mode inductor: Modeling and design issues," IEEE Transactions on Industrial Electronics, vol. 58, no. 8, pp. 3264-3274, 2011.

[75] F. Traub, B. Wunsch, and S. Skibin, "A high frequency model of toroidal chokes for EMC filtering," in IEEE International Symposium on Electromagnetic Compatibility. Dresden: IEEE, 2015, pp. 902-907. 
[76] C. Dominguez-Palacios, J. Bernal Mendez, and M. A. Martin Prats, "Characterization of Three-Phase Common Mode Chokes at High Frequencies," IEEE Transactions on Power Electronics, vol. 33, no. 5, pp. 3975$3987,2018$.

[77] S. Weber, M. Schinkel, S. Guttowski, W. John, and H. Reichl, "Calculating Parasitic Capacitance of Three-Phase Common-Mode Chokes," Power Conversion Intelligent Motion (PCIM), pp. 1-6, 2005.

[78] A. Massarini and M. K. Kazimierczuk, "Self-capacitance of inductors," IEEE Transactions on Power Electronics, vol. 12, no. 4, pp. 671-676, 1997.

[79] R. Feynman, R. Leighton, and M. Sands, Feynman Lectures on Physics. Addison-Wesley Publishing Company, 1970, vol. II.

[80] G. Asmanis, L. Ribickis, and A. Asmanis, "Modeling of Mutual Coupling between Inductors," in Electromagnetic Compatibility and Signal Integrity, 2015 IEEE Symposium on, 2015, pp. 294-299.

[81] K. Kostov and J. Kyyrä, "Common-Mode Choke Coils Characterization," in Proceedings of the 13th European Conference on Power Electronics and Applications (EPE 2009), Barcelona, Spain, 2009.

[82] T. Weiland, M. Timm, and I. Munteanu, "A practical guide to 3-D simulation," IEEE Microwave Magazine, vol. 9, no. 6, pp. 62-75, 2008.

[83] R. Huang and D. Zhang, "Characterization of Mn - Zn Ferrites Using the Coaxial Transmission Line Method," IEEE Transactions on Magnetics, vol. 44, no. 7, pp. 1703-1710, 2008.

[84] J. Xu, M. Koledintseva, and Y. Zhang, "Complex permittivity and permeability measurements and finite-difference time-domain simulation of ferrite materials," IEEE Transactions on Electromagnetic Compatibility, vol. 52, no. 4 , pp. 878-887, 2010.

[85] W. Li, X. Wang, D. Liang, and L. Deng, "Low Frequency $(100 \mathrm{kHz}-1$ $\mathrm{MHz}$ ) Permeability Measurement," in Progress In Electromagnetic Research Symposium (PIERS), Shanghai, 2016.

[86] C. Cuellar, W. Tan, X. Margueron, A. Benabou, and N. Idir, "Measurement method of the complex magnetic permeability of ferrites in high frequency," in 2012 IEEE International Instrumentation and Measurement Technology Conference Proceedings, Graz, Austria, 2012, pp. 63-68.

[87] J. Lansink Rotgerink, J. Verpoorte, and H. Schippers, "Uncertainties in coaxial cable transfer impedance," IEEE Electromagnetic Compatibility Magazine, vol. 7, no. 3, pp. 83-93, 2018. 
[88] A. Roc'h and F. Leferink, "Optimization of the in situ Performance of Common Mode Chokes for Power Drive Systems Using Designable Parameters," IEEE Transactions on Electromagnetic Compatibility, vol. 60, no. 2, pp. 1-7, 2018.

[89] S. Wang, S. Member, F. C. Lee, D. Y. Chen, and W. G. Odendaal, "Effects of Parasitic Parameters on EMI Filter Performance," IEEE Transactions on Power Electronics, vol. 19, no. 3, pp. 869-877, 2004.

[90] T. D. E. Oliveira, J.-1. Schanen, J.-m. Guichon, L. Gerbaud, G. U. M. R. Inpg-ujf cnrs, B. Ense, S. Martin, and C. France, "Automatic Layout Optimization of an EMC filter," Energy Conversion Congress and Exposition (ECCE), 2010 IEEE, pp. 2679-2685, 2010.

[91] F. Zare, "EMI issues in modern power electronic systems," The IEEE EMC Society Newsletters, no. 221, pp. 66-70, 2009.

[92] R. Aguilera, P. Acuna, P. Lezana, G. Konstantinou, B. Wu, S. Bernet, and V. Agelidis, "Selective Harmonic Elimination Model Predictive Control for Multilevel Power Converters," IEEE Transactions on Power Electronics, vol. PP, no. 99, pp. 1-1, 2016.

[93] J. Muñoz, I. Torres, J. Guzmán, C. Baier, P. Melin, J. Rohten, U. Bío-bío, J. Espinoza, and J. Silva, "Selective Harmonic Elimination for a 27-Level Asymmetric Multilevel Converter," in Environment and Electrical Engineering and 2017 IEEE Industrial and Commercial Power Systems Europe (EEEIC/I \&CPS Europe), 2017 IEEE International Conference on. IEEE, 2017, pp. 7-11.

[94] Keysight E5061B Online Manual, "http://ena.support.keysight.com/e5061b/manuals/webhelp/eng/?nid=11143.0.00\&cc $=$ NL\&lc $=$ dut\&id $=1790874 . "$

[95] C. Keyer, F. Buesink, and F. Leferink, "Mains Power Synchronous Conducted Noise Measurement in the 2 to $150 \mathrm{kHz}$ band," in Electromagnetic Compatibility (EMC EUROPE), 2016 International Symposium on, Wroclaw, 2016, pp. 865-869.

[96] T. Hartman, N. Moonen, and F. Leferink, "Evaluation of Multichannel Synchronous Conducted TDEMI Measurements for High Voltage Power Electronics," in 2018 International Symposium on Electromagnetic Compatibility (EMC EUROPE). Amsterdam: IEEE, aug 2018, pp. 839-843. 


\section{List of my Publications}

[1] N. Moonen, F. Buesink, and F. Leferink, "Unexpected poor performance of presumed high-quality power line filter, and how it improved," in 2015 IEEE International Symposium on Electromagnetic Compatibility (EMC). Dresden: IEEE, aug 2015. doi: 10.1109/ISEMC.2015.7256191. ISBN 9781-4799-6616-5. ISSN 21581118 pp. 382-385.

[2] —_, "Enhanced circuit simulation using mutual coupling parameters obtained via 3D field extraction," in 2016 Asia-Pacific International Symposium on Electromagnetic Compatibility (APEMC). Shenzhen, China: IEEE, may 2016. doi: 10.1109/APEMC.2016.7523002. ISBN 978-1-46739494-9 pp. 181-183.

[3] — - "Current Barriers to Confine High Frequency Common Mode Currents," in International Symposium on Electromagnetic Compatibility (EMC EUROPE). Wroclaw: IEEE, 2016. doi: 10.1109/EMCEurope.2016.7739295. ISBN 9781509014163 . ISSN 21581118 pp. 586-589.

[4] — - "Optimizing capacitor placement in EMI-filter using back annotation of 3D field coupling parameters in circuit models," in 2016 International Symposium on Electromagnetic Compatibility - EMC EUROPE. Wroclaw: IEEE, sep 2016. doi: 10.1109/EMCEurope.2016.7739225. ISBN 978-1-5090-1416-3. ISSN 21581118 pp. 576-580.

[5] N. Moonen, M. Gagic, F. Buesink, J. A. Ferreira, and F. Leferink, "Harmonic cancellation in a novel multilevel converter topology for the future smart grid," in 2017 IEEE International Symposium on Electromagnetic Compatibility $\mathcal{E}$ Signal/Power Integrity (EMCSI). Washington: IEEE, aug 2017. doi: 10.1109/ISEMC.2017.8077939. ISBN 978-1-5386-2229-2. ISSN 21581118 pp. 602-607.

[6] N. Moonen, F. Buesink, and F. Leferink, "EMI reduction in sPWM Driven $\mathrm{SiC}$ converter based on carrier frequency selection," in 2017 International Symposium on Electromagnetic Compatibility - EMC EUROPE. Angers: IEEE, sep 2017. doi: 10.1109/EMCEurope.2017.8094755. ISBN 978-15386-0689-6 pp. $1-5$. 
[7] N. Moonen, J. L. Rotgerink, and F. Leferink, "Automated equivalent circuit extraction of impedance curves using a Gauss-Newton algorithm," in 2018 IEEE International Symposium on Electromagnetic Compatibility and 2018 IEEE Asia-Pacific Symposium on Electromagnetic Compatibility (EMC/APEMC). Singapore: IEEE, may 2018. doi: 10.1109/ISEMC.2018.8393958. ISBN 978-1-5090-5997-3 pp. 1103-1108.

[8] N. Moonen, A. Roc'h, and F. Leferink, "Influence of mutual coupling on parasitic capacitance in common mode chokes," in 2018 IEEE International Symposium on Electromagnetic Compatibility and 2018 IEEE AsiaPacific Symposium on Electromagnetic Compatibility (EMC/APEMC). Singapore: IEEE, may 2018. doi: 10.1109/ISEMC.2018.8394000. ISBN 978-1-5090-5997-3 pp. 1305-1309.

[9] N. Moonen, R. Vogt-Ardatjew, A. Roc'h, and F. Leferink, "3-D Full-Wave High Frequency Common Mode Choke Modeling," IEEE Transactions on Electromagnetic Compatibility, no. (Early Access), pp. 1-8, 2019. doi: 10.1109/TEMC.2019.2914371

[10] D. Mandaris, N. Moonen, S. van de Beek, F. Buesink, and F. Leferink, "Validation of a Fully Anechoic Chamber," in AsiaPacific International Symposium on Electromagnetic Compatibility 8 Signal Integrity (APEMC). Shenzhen, China: IEEE, 2016. doi: 10.1109/APEMC.2016.7522892. ISBN 9781467394949 pp. 865-868.

[11] D. Mandaris, N. Moonen, J. Schuurmans, and F. Leferink, "Comparison of log-periodic, dual-stacked log-periodic, and horn antenna on the field uniformity and power efficiency from $80 \mathrm{MHz}$ to $1 \mathrm{GHz}$," in 2017 Asia-Pacific International Symposium on Electromagnetic Compatibility (APEMC). Seoul: IEEE, jun 2017. doi: 10.1109/APEMC.2017.7975443. ISBN 978-1-5386-3912-2 pp. 127-130.

[12] I. Setiawan, N. Moonen, F. Buesink, and F. Leferink, "Efficient magnetic field measurements," in 2017 International Symposium on Electromagnetic Compatibility - EMC EUROPE. IEEE, sep 2017. doi: 10.1109/EMCEurope.2017.8094823. ISBN 978-1-5386-0689-6 pp. 1-4.

[13] T. Hartman, N. Moonen, and F. Leferink, "Evaluation of Multichannel Synchronous Conducted TDEMI Measurements for High Voltage Power Electronics," in 2018 International Symposium on Electromagnetic Compatibility (EMC EUROPE). Amsterdam: IEEE, aug 2018. doi: 10.1109/EMCEurope.2018.8485175. ISBN 978-1-4673-9698-1. ISSN 21581118 pp. $839-843$.

[14] — , "RFI Estimation from Non-GSO Satellites Based on Two Line Element Assisted Equivalent Power Flux Density Calculations," in 2018 International Symposium on Electromagnetic Compatibility (EMC EUROPE). 
Amsterdam: IEEE, aug 2018. doi: 10.1109/EMCEurope.2018.8485076. ISBN 978-1-4673-9698-1. ISSN 21581118 pp. 17-21.

[15] — , "Direct Sampling in Multi-channel Synchronous TDEMI Measurements," in 2018 IEEE 4th Global Electromagnetic Compatibility Conference (GEMCCON). Stellenbosch: IEEE, nov 2018. doi: 10.1109/GEMCCON.2018.8628576. ISBN 978-1-5386-5728-7 pp. 1-5.

[16] C. Van Diemen, N. Moonen, and F. Leferink, "Estimation of Radiation Efficiency of GaN Half-Bridge Based Submodule System for Radiated EMI Prediction," in 2018 International Symposium on Electromagnetic Compatibility (EMC EUROPE), First Runner-Up Best Student Paper Award. Amsterdam: IEEE, aug 2018. doi: 10.1109/EMCEurope.2018.8485132. ISBN 978-1-4673-9698-1. ISSN 21581118 pp. 102-107.

[17] S. Isrie, N. Moonen, H. Schipper, H. Bergsma, and F. Lefcrink, "Measuring, Logging, and Visualizing Pulsed Electromagnetic Fields Combined with GPS Location Information," in 2018 International Symposium on Electromagnetic Compatibility (EMC EUROPE). Amsterdam: IEEE, aug 2018. doi: 10.1109/EMCEurope.2018.8485116. ISBN 978-1-4673-9698-1. ISSN 21581118 pp. 500-505.

[18] B. ten Have, T. Hartman, N. Moonen, C. Keyer, and F. Leferink, "Faulty Readings of Static Energy Meters Caused by Conducted Electromagnetic Interference from a Water Pump," in International Conference on Renewable Energies and Power Quality (ICREPQ'19), Tenerife, 2019.

[19] B. Have, T. Hartman, N. Moonen, and F. Leferink, "Misreadings of Static Energy Meters due to Conducted EMI caused by Fast Changing Current," in Asia-Pacific International Symposium on Electromagnetic Compatibility \& Signal Integrity (APEMC). Sapporo: IEEE, 2019, pp. 445-448.

[20] T. Hartman, N. Moonen, B. ten Have, and F. Leferink, "Fast Magnetic Emission Tests for Continuous Measurements Around an Equipment Under Test," in ESA Workshop on Aerospace EMC. Budapest: IEEE, 2019.

[21] J. Lansink Rotgerink, N. Moonen, and F. Leferink, "Mixed-Mode SParameter Measurements for Determination of Cable Coupling," in ESA Workshop on Aerospace EMC. Budapest: IEEE, 2018.

[22] M. Appelman, M. A. Wibisono, W. Shalannanda, N. Moonen, and F. Leferink, "Comparison of Time-domain Measurement Techniques for Interference Analysis in Power Line Communication," in International Conference on Wireless and Telematics (ICWT). Yogyakarta: IEEE, 2019. ISBN 9781538661635

[23] B. ten Have, T. Hartman, N. Moonen, and F. Leferink, "Inclination of Fast Changing Currents Effect the Readings of Static Energy Meters," in International Symposium on Electromagnetic Compatibility (EMC EUROPE). Barcelona, Spain: IEEE, 2019. 
[24] — - "Why Frequency Domain Tests Like IEC 61000-4-19 Are Not Valid ; a Call For Time Domain Testing," in International Symposium on Electromagnetic Compatibility (EMC EUROPE). Barcelona, Spain: IEEE, 2019.

[25] P. Lezynski, R. Smolenski, H. Loschi, D. Thomas, and N. Moonen, "A novel method for EMI evaluation in random modulated power electronic converters," Elsevier Measurement Journal, vol. TBD, no. TBD, p. Accepted for publication, 2019. 


\section{Appendix A}

\section{Protection Circuit}

The Nexys3 FPGA board is used as operation control board. During the uploading process of the FPGA, all the logical outputs go high. During automated measurements of a half-bridge, this would result in a short-circuit fault. This would result in high currents during the connection to the grid that might destroy the switches. Therefore a simple protective circuit was designed, that acts as a pass-through for the control signals unless when both are in high state. Then the outputs will be inverted, and thus no short circuit fault will occur. Figure A.1 shows for a single SM the circuit used, while Table A.1 shows the resulting outputs for all possible combinations of input signals. The circuit is designed using the following components:

- XOR - CD74HC08E

- AND - SN74HC86N

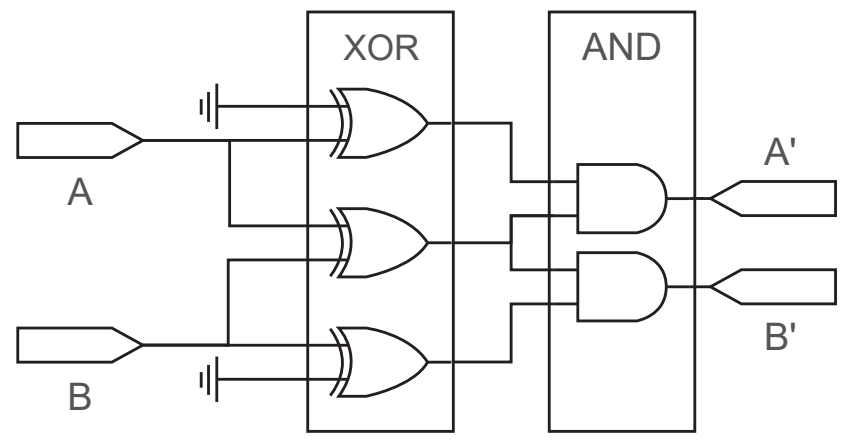

Figure A.1: Logical protection circuit

Extensive amount of measurements have been performed varying the correct operation of the control board with and without the protection circuit connected. As can be seen from Figure A.2, the protection board decreases 
Table A.1: Truth Table of Protection Circuit

\begin{tabular}{|cc|cc|}
\hline $\mathrm{A}$ & $\mathrm{B}$ & $\mathrm{A}^{\prime}$ & $\mathrm{B}^{\prime}$ \\
\hline 0 & 0 & 0 & 0 \\
0 & 1 & 0 & 1 \\
1 & 0 & 1 & 0 \\
1 & 1 & 0 & 0 \\
\hline
\end{tabular}

the fall and rise times significantly. The direct output of the FPGA evaluation board is providing logical signaling with rise and fall times between 9 ns and $16 \mathrm{~ns}$, while the protective circuit provides signaling between $3 \mathrm{~ns}$ and $4.4 \mathrm{~ns}$. COTS available GaN and $\mathrm{SiC}$ switches can have transition speeds in this order of several nanoseconds.

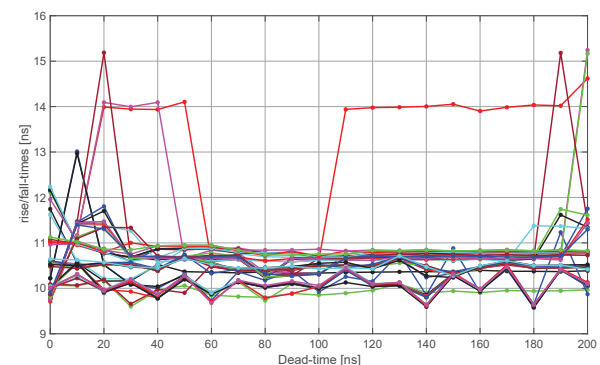

(a) Direct board output

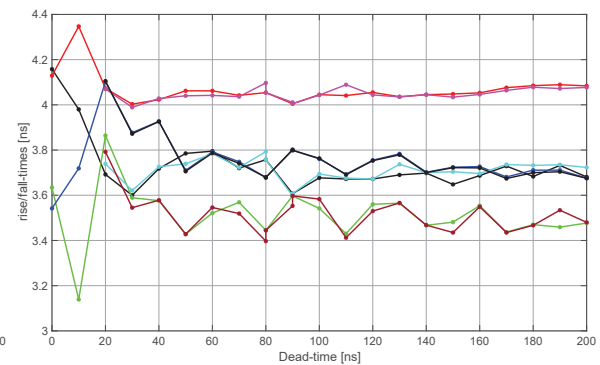

(b) Through protection circuit

Figure A.2: Measurements of Nexys3 logical Rise and Fall times, for varying dead-times, and output frequencies 


\section{Appendix B}

\section{Impedance Measurement ENA}

Throughout this thesis, many times impedance measurements have been performed using the E5061A. Here in this Appendix the procedure used to measure them is explained.

\section{B.1 E5061B}

Using the Keysight E5061B ENA (Figure B.1) in conjunction with the impedance analyzer options allows for using spring clip fixture 16092A (Figure B.2) in accurate impedance measurements from $5 \mathrm{~Hz}$ to approx. $500 \mathrm{MHz}$. In our case the spring clip fixture is used in conjunction with the HP 41951-69001 Impedance Test Adapter (Figure B.3d). It is based on the already documented steps of impedance measurement in any situation.

The HP 41951-69001 has a reflection and transmission port, as well as an input and bias port. This allows for large range of possible investigations. We have used it to determine impedances of ferrites and extract complex permeability, but it is not limited to this. Figure B.4a shows the DUT impedance regions, and which method is most useful/accurate for measuring this. In case of the HP 16092A, the transmission port will be used of Port 1-2 series measurements, while the reflection port can be used for the Port 1-2 shunt measurement. Note however that the other port should always be terminated with a broadband impedance of $50 \Omega$.

The calibration stage consists of 2 steps, first the HP 41951-69001 test adapter is calibrated using a standard $7 \mathrm{~mm}$ open/short/load calibration kit, which can be seen in Figure B.3. While afterwards the HP 16092A spring clip fixture is added, and fixture compensation can be used to remove its influence. Which again is a open,short and load calibration. However no standard kit is available. The open is quite obvious, however using a wire and leaded resistor (or something similar) will add parasitics to the calibration of which 
the user should be aware of. Using SMD type of $50 \Omega$ resistor will most likely allow for the most accurate calibration, as most influential parasitics will be insignificant up until the upper limit of the analyzer $(3 \mathrm{GHz})$. The short can be accomplished by using the movable part of the spring clip fixture, but should be done prior to open calibration as one doesn't want to move this part after the open measurement was done.

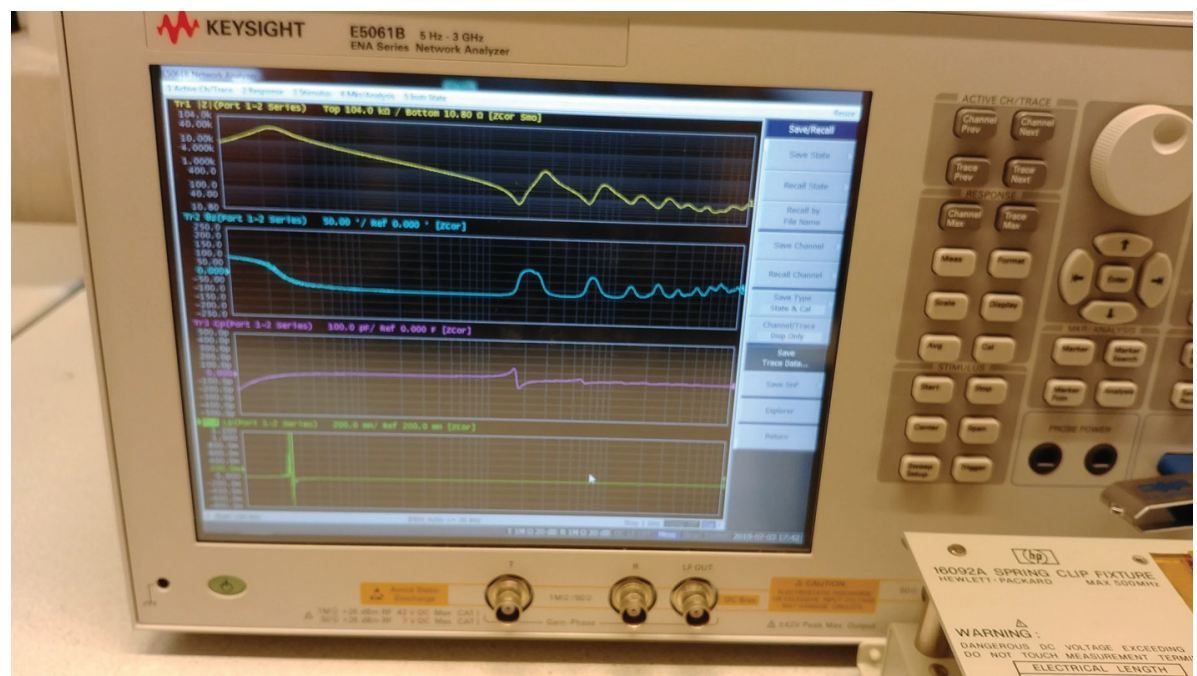

Figure B.1: Keysight 5061B ENA in use. 


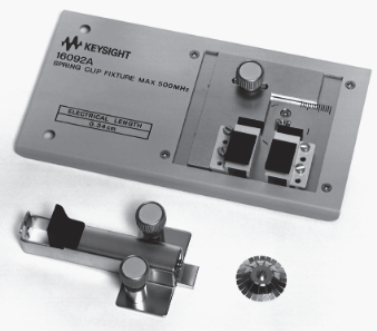

(a)

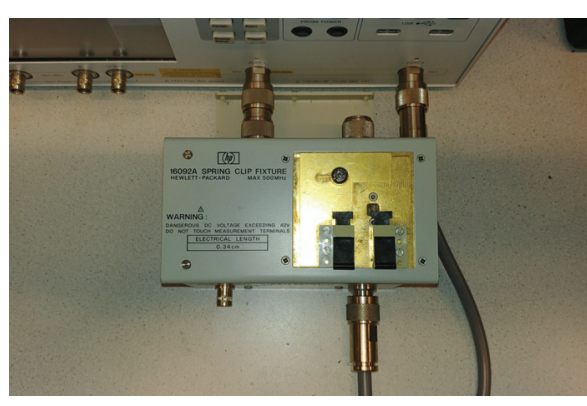

(b) Implemented spring clip fixture HP 16092A

Figure B.2: Spring clip fixture and its implementation with a E5061B ENA and HP 41951-69001 impedance test adapter.

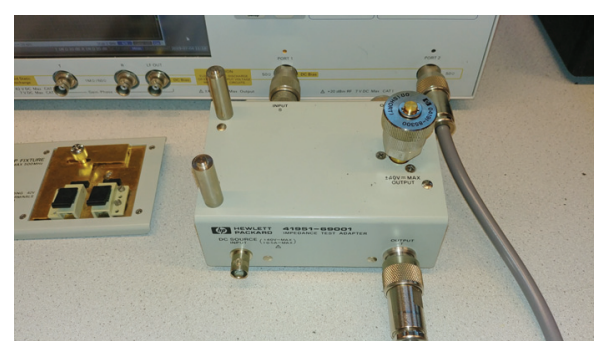

(a) Short

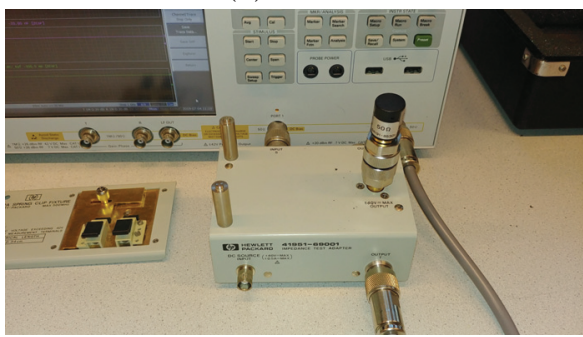

(c) Load

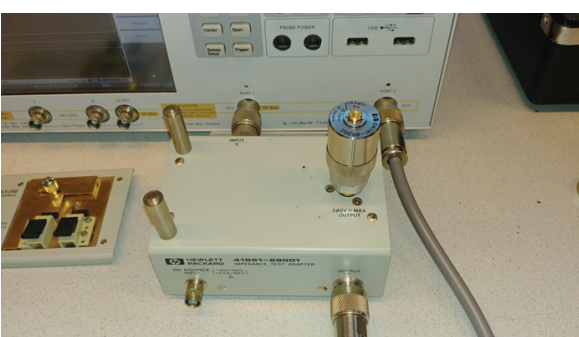

(b) Open

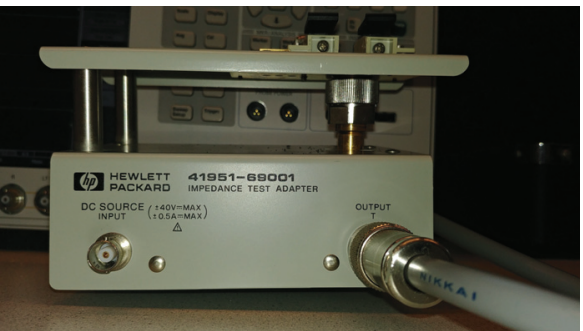

(d) Calibration Plane

Figure B.3: Open, short and load calibration of impedance testing adapter in Port 1-2 Series mode. 


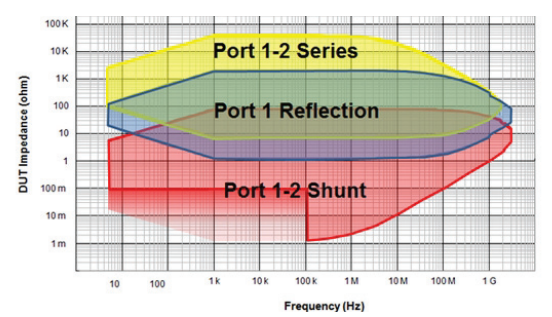

(a) DUT impedance ranges with $10 \%$ accuracy per measurement method.

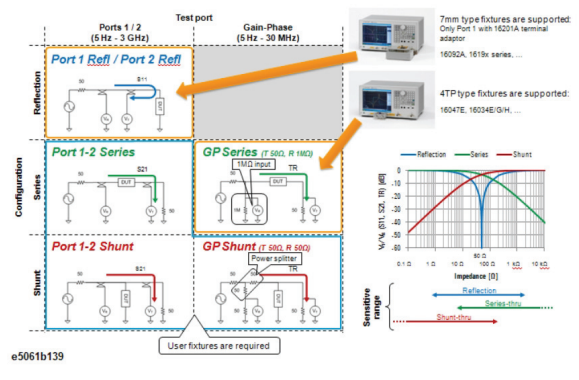

(b) Configurations of measurements.

\begin{tabular}{|c|c|c|c|c|c|}
\hline Method & Port 1 (or 2) Reflection & Port 1-2 Series & Port 1-2 Shunt & GP Series & GP Shunt \\
\hline $\begin{array}{l}\text { Measurement Dut Impedance } \\
\text { Range }\end{array}$ & Low to middele impedance & $\begin{array}{l}\text { Middle to high Impedance in the } \\
\text { high trequench range } \\
\text { Not applicable to grounded DUTS }\end{array}$ & $\begin{array}{l}\text { Very low Impedance in the high } \\
\text { frequency ranges }\end{array}$ & $\begin{array}{l}\text { Middle to high Impedance in the low } \\
\text { frequency range }\end{array}$ & $\begin{array}{l}\text { Very low Impedance in the low. } \\
\text { frequency range }\end{array}$ \\
\hline Formula & zdut $=50 \times(1+511) /(1-511)$ & zdut $=50 \times 2 \times(1-521) / 521$ & Zdut $=50 \times 521 /(2 \times(1-521))$ & $\begin{array}{l}Z \text { dut }=50 \times(1-T \mathrm{~T}) / T \mathrm{R} \\
\mathrm{TR}=T / \mathrm{R}\end{array}$ & 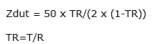 \\
\hline
\end{tabular}

(c) Characteristics of the measurement methods.

\begin{tabular}{|c|c|c|c|c|c|c|}
\hline Method & Frequency & Calibration & IFBW & & Source Power & Note \\
\hline \multirow{3}{*}{ Port 1-2 Senes } & \multirow{5}{*}{$5 \mathrm{~Hz}$ to $3 \mathrm{GHz}$} & \multirow{3}{*}{ 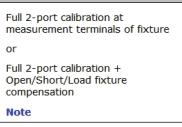 } & \multicolumn{2}{|c|}{ See the following table } & \multirow{5}{*}{-20 to o dem } & \\
\hline & & & \begin{tabular}{|l}
$\begin{array}{l}\text { Measurement } \\
\text { Frequency }\end{array}$ \\
\end{tabular} & IF BW & & \\
\hline & & & $<200 \mathrm{~Hz}$ & 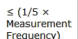 & & \\
\hline \multirow{2}{*}{ Port 1 Reffection } & & \multirow{2}{*}{ 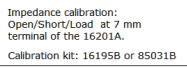 } & & Hz= & & \\
\hline & & & $\geq 200 \mathrm{~Hz}$ & & & \\
\hline Port 1-2 Shunt & $100 \mathrm{kHz}$ to $3 \mathrm{GHz}$ & $\begin{array}{l}\text { Impedance callbration: } \\
\text { Open//5hort/Load } \\
\text { Note }\end{array}$ & $10 \mathrm{~Hz}$ & & $10 \mathrm{dBm}$ & 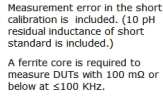 \\
\hline
\end{tabular}

(d) Conditions where the $10 \%$ measurement accuracy range shown in Figure B.4a is specified.

Figure B.4: Manual info, adopted from [94] 


\section{Appendix $\mathrm{C}$}

\section{Processing Radiation}

Radiated emission testing is done in this thesis to characterize a single SM. Figure 5.9a shows the basic setup and processing steps. In this appendix the more practical details of the digital signal process is described. As this is mostly textbook material it is omitted as core material of the thesis, however it is important for discussing the results and their implications.

The goal of the signal processing is discussed in chapter 5. By assuming a known input signal and known output signal one would be able to calculate the transfer function of any LTI system.

$$
H(s)=\frac{Y(s)}{X(s)}
$$

Which in case of antennas translates into a radiation efficiency:

$$
\eta(\omega)=\frac{P_{\text {rad }}(\omega)}{P_{\text {sup }}(\omega)}
$$

$P_{\text {sup }}$ is effectively the power supplied to a unknown system, and the $P_{\text {rad }}$ the radiated power by this system. In case of Figure $5.9 \mathrm{a}, x(t)$ is the known voltage supplied and $y(t)$ the recorded waveform from the oscilloscope. The following section describes the procedure to calculate the wanted powers from these signals, thus: $x(t) \rightarrow P_{\text {sup }}$ and $y(t) \rightarrow P_{\text {rad }}$

\section{C.1 Received signal $y(t)$}

In Figure 5.9a the signal $y(t)$ is recorded using an oscilloscope and the start of the applied DSP.

$$
\mathcal{F}[y(t) \cdot w(t)]=Y(\omega)
$$

with $w(t)$ being a time windowing function which is necessary in performing an Fast Fourier Transform (FFT) over a non-integer number of cycles. In case of the results presented in this thesis a Hann window was chosen, as it has very 
low aliasing with a slight trade-off in decreasing the resolution. Its discrete version is denoted as

$$
w[n]=\sin ^{2}\left(\frac{\pi n}{N-1}\right)
$$

with $N$ being the total amount of samples. For conservation of power, the resulting signal needs to be corrected using a windowing correction factor. In case of the Hann window the amplitude correction factor is 2. Next to correction for windowing, the output needs to be normalized by the amount of samples in which the time-signal $y(t)$ is recorded. By correctly applying these processing steps the resulting $Y(\omega)$ has units of voltage similar to the input.

The voltage is recorded at the output of an antenna, thus is proportional/related to the electric field measured by it. The antenna acts as a transducer for fields, using the calibration data one is able to acquire fields strength values according to:

$$
E\left[\mathrm{dbVm}^{-1}\right]=V_{0}[\mathrm{dBV}]+A F E\left[\mathrm{dBm}^{-1}\right]
$$

Continuing to obtain $P_{\text {rad }}$ from the field-strength, a few assumptions need to be made. The measurements were performed in a situation that represents free space (i.e. wave impedance is $\eta=120 \pi$ ) and the radiator under test is considered isotropic (i.e. the power is distributed uniformly over a sphere). Using the Poynting's theorem the relation can be found as:

$$
\begin{gathered}
P_{\text {rad }}=\frac{E^{2} \cdot r^{2}}{30} \quad\left[\frac{\frac{\mathrm{V}^{2}}{\mathrm{~m}^{2}} \cdot \mathrm{m}^{2}}{\Omega}\right] \\
P_{\text {dens }}=E \times H \\
\left|P_{\text {dens }}\right|=E \cdot H=\frac{E^{2}}{\eta} \\
P_{\text {dens }}=\frac{P_{\text {rad }}}{4 \pi r^{2}}
\end{gathered}
$$

\section{C.2 Supplied signal $x(t)$}

The supplied signal is described in Chapter 2, in this thesis it is the result of the mathematical model:

$$
S_{d e m} \approx \sum_{m=1}^{\infty} \frac{k V_{p-p}}{m \pi} \sqrt{\frac{2 k}{m \pi^{2} M}} \operatorname{rect}\left(\frac{f-m f_{c}}{f_{m} m \pi M+2 f_{m}}\right)
$$

In this case, this is the amplitude spectrum in which case the dimension is voltage. Only by applying this to a load with a known impedance one can relate this to $P_{\text {sup }}$. 
Table C.1: Settings for EMI test receiver in case of RE101

\begin{tabular}{c|c|c}
\hline Freq. Range & Bandwidth & Step Size \\
\hline $30 \mathrm{~Hz}-1 \mathrm{kHz}$ & $10 \mathrm{~Hz}$ & $5 \mathrm{~Hz}$ \\
$1 \mathrm{kHz}-10 \mathrm{kHz}$ & $100 \mathrm{~Hz}$ & $50 \mathrm{~Hz}$ \\
$10 \mathrm{kHz}-100 \mathrm{kHz}$ & $1 \mathrm{kHz}$ & $500 \mathrm{~Hz}$
\end{tabular}

\section{C.3 Magnetic field}

\section{C.3.1 RE101}

The digital signal processing done for this work, is related to the MIL-STD RE101. We want to examine the relation between transients and B-fields in time, however the recorded signal is always a time varying voltage. The recorded signal $\mathrm{x}(\mathrm{t})$, should first be transformed into a spectrum in $\mathrm{dB} \mu \mathrm{V}$, to which the antenna factor can be applied. Using the Short Time Fast Fourier Transform (STFFT) one can, through digital signal processing, mimic many parallel connect EMI test receivers all tuned to a different band. The basic operation of STFFT can be described with:

$$
X(m, \omega)=\sum_{n=-\infty}^{\infty} x[n] w[n-m] \exp (-j \omega n)
$$

As a result, one can recreate different types of EMI detectors by applying functions as averaging or selecting the maximum value over the dimension $m$ of $|X(m, \omega)|$, resulting in $X_{a v g}(\omega)$ and $X_{p k}(\omega)$.

In case of the processing done for the RE101 magnetic field test, there are some requirements on the resulting spectrum summarized in Table C.1. In which case the bandwidth is related to the windowing function and overlap used in the STFFT, while the step size basically is frequency resolution $(\Delta f)$. In creating the spectrogram, there exists a trade-off between frequency resolution and time resolution, which can be seen as an inverse proportionality: $\Delta f \propto \frac{1}{\Delta t}$

From $X_{a v g}(\omega)$ and $X_{p k}(\omega)$ one can then derive the single sided power spectra. If correctly applied, one ends up with $\mathrm{dB} \mu \mathrm{V}$ similar and comparable to the output of the EMI test receiver. Note that deviations can exist though, as the receivers are operating under the condition the disturbance is periodic.

\section{C.3.2 Time domain}

Measuring the magnetic field over time using a loop antenna requires some postprocessing to recover the actual signal as opposed to the measured waveform. The time variant magnetic field can be recovered via Fourier transform and its 
inverse, while applying the antenna factor to account for the loop antenna used in frequency domain.

$$
\begin{gathered}
\mathcal{F}[x(t)]=X(\omega), \quad Y(\omega)=X(\omega) \cdot A F(\omega) \\
\mathcal{F}^{-1}[(Y(\omega))]=y(t)
\end{gathered}
$$

With $\mathrm{x}(\mathrm{t})$ being the recorded voltage from the loop antenna, $\mathrm{y}(\mathrm{t})$ the magnetic field measured, and AF the antenna factor. Performing these operations will result in a complex valued time series, of which only the real values have meaning. The imaginary part is a result of rounding errors and applying a perfect filter for frequencies above $100 \mathrm{kHz}$. Assuming a perfect symmetrical spectrum around the Nyquist frequency removes this issue. 


\section{Appendix D}

\section{Heater as load}

A resistive heater has been used in this thesis as a load for many of the measurement setups, as this was expected to act as a broadband resistor for the operating frequencies of the power converters. Also the resistance is variable, which is related to the amount of heat it produces. The six different power settings can be seen in the legend of Figure D.1, it ranges from $190 \mathrm{~W}$ to $1800 \mathrm{~W}$. The impedance was measured at each of these settings for different frequencies. Even though the impedance increases slightly with increasing frequencies, the measurements show that the heater can be considered to be a broadband resistor.

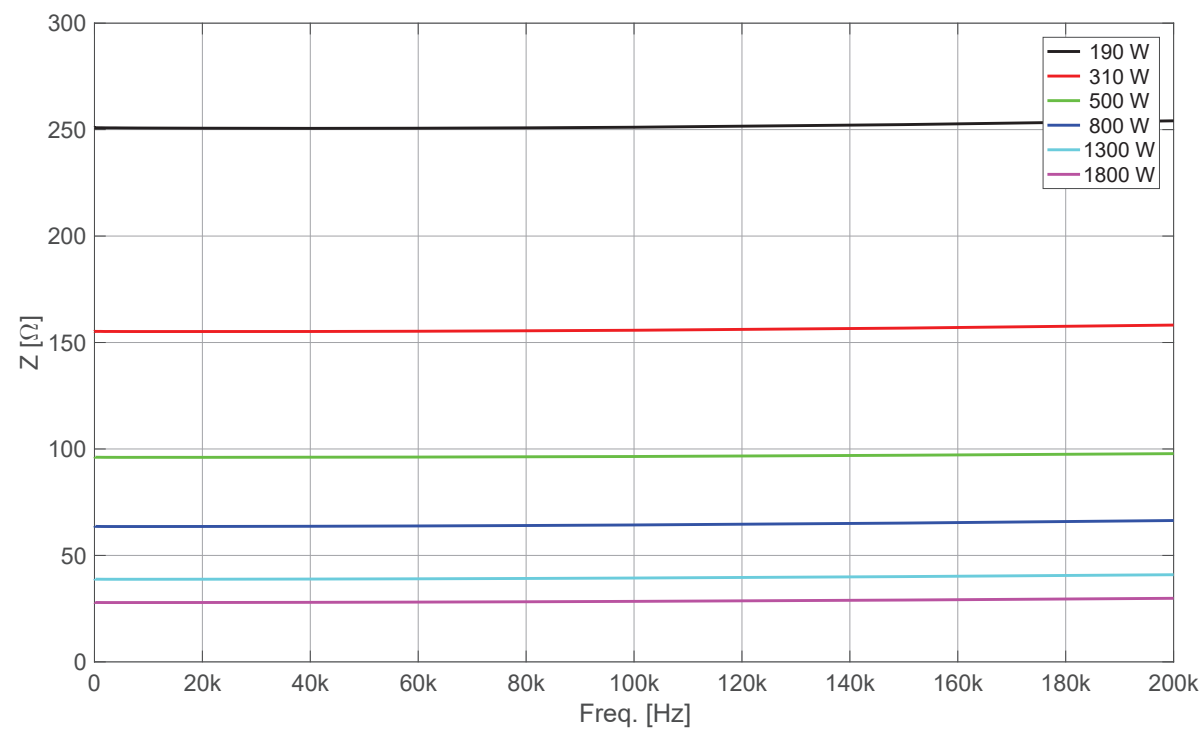

Figure D.1: Impedance measurement of heater with cable 


\section{Appendix E}

\section{Permeability}

Lots of research exist into the development of different core materials. The design usually ended in a trade-off of effective permeability vs the maximum magnetic saturation flux density. The latest developments include laminated nano-crystalline material, which is using the best of both worlds. The magnetic permeability is the main property of the core material that influences the overall performance of a CMC. It is a crucial component in accurately modeling an inductor's high frequency behavior, and thus its parasitic behavior. [80] has shown excellent results in inductor modeling using precise magnetic material characteristics, i.e. frequency dependent conductivity, permitivity and complex magnetic permeability, which are often unavailable or protected by intellectual property. We try to solve this problem and compare three methods for obtaining the permeability:

- Data sheet

- Measurement of the core

- Measurement of the choke/inductor

This will result in a discussion on the benefits and challenges arising from these methods.

\section{E.1 Data sheet}

Data sheet values have a challenge of often being limited at their frequency range to be used and the accuracy during the data extraction method. Either reading or a plot extraction method to acquire data-points. Hence, two methods of obtaining the permeability from data sheets are identified. The direct method, using the core-material data sheet. The indirect method, using the attenuation, impedance or equivalent measurements of the used inductor type component. 


\section{E.1.1 Direct}

In the case of winding a custom inductor or even a CMC, often a core is used with a "known" permeability is used. Often only single valued permeabilities are used in calculation and thus these are most often available. In some cases a frequency dependent complex permeability is given. Figure 4.21a shows this for a COTS available magnetic core named: "TX22/14/6.4-3E27".

\section{E.1.2 Indirect}

Often the case with data sheets of CMC's, the permeability is not shown, but the insertion loss or attenuation profile. These measurements of the manufacturer are directly related to the impedance. In case of toroids, the relation between impedance and complex permeability of the core material is shown in section E.3. Therefore the relation between insertion loss and impedance is shown here, and verified to be useful to derive the absolute permeability, due to the fact the data sheet provides only magnitude values. Verification will be done comparing impedances (Figure 4.25a). from insertion loss:

$$
I L=-20 \log \left|S_{21}\right|, \quad Z=50 \cdot \frac{2\left(1-S_{21}\right)}{S_{21}}
$$

\section{E.2 Core measurement}

Accurately measuring a core's complex magnetic permeability often posses quite some challenges. It requires specialized equipment, or is dependent on a large number of parasitic influences. [83-86]

\section{E.2.1 Adapter usage}

The adapter used was based on [85]. It showed a coaxial magnetic cavity measurement for determining the complex permeability at low frequencies $(100 \mathrm{kHz}$ - $1 \mathrm{MHz}$ ). Following their methodology, our measurement setup was used from $(1 \mathrm{kHz}-3 \mathrm{GHz})$. Using Equation E.1 together with $\mathcal{Z}=Z_{\text {core }}-Z_{\text {empty }}$ and $N=1$ gives the results shown in Figure E.2.

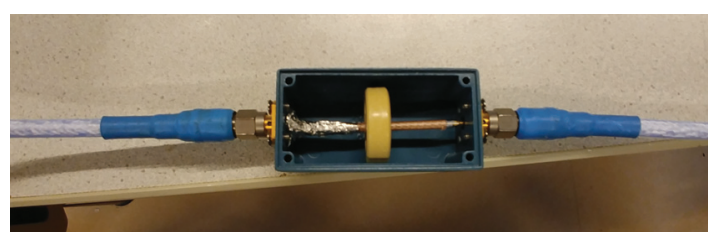

(a) Adapter measurement 


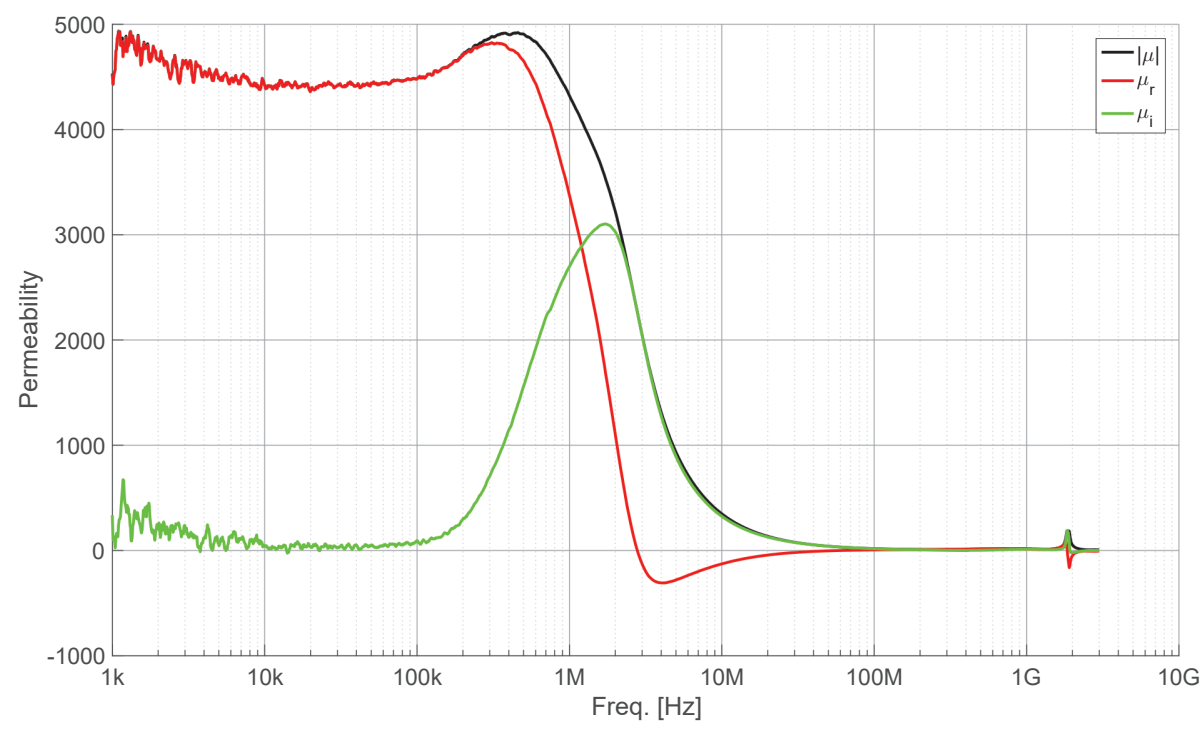

Figure E.2: Permeability of Ferroxcube - TX22/14/6.4-3E27 from adapter measurement

\section{E.3 Inductor measurement}

Using a VNA/Impedance analyzer measuring the complex impedance, one can derive the complex permeability based on the shape of the core material. In case of toroidal shapes we know from [79] the following:

$$
\mathscr{L}=\frac{\mu N^{2} A}{l}
$$

With:

$$
l=\frac{\pi\left(d_{o}-d_{i}\right)}{\ln \frac{d_{o}}{d_{i}}}, \quad A=h \cdot \frac{\left(d_{o}-d_{i}\right)}{2}
$$

$l$ is the average length of the magnetic field lines inside the toroid. Assumed here is that the material used is homogeneous and thus the field $\mathbf{H}$ is uniform in the toroidal shaped core. from this follows:

$$
\mathscr{L}=\frac{\mu_{0} \mu_{r} N^{2} h \ln \frac{d_{o}}{d_{i}}}{2 \pi}(\text { toroid })
$$

Here it is assumed that $\mu$ is real valued and the measured toroid to be a perfect inductor with only an inductance. Replacing the inductance with complex impedance shows directly that the permeability is also complex and frequency dependent. With $Z \propto j \omega L$ it follows follows:

$$
\mu_{r}=\frac{2 \pi \mathcal{Z}}{j \mu_{0} \omega N^{2} h \ln \frac{d_{o}}{d_{i}}}=\frac{\mathcal{Z}}{j \mu_{0} f N^{2} h \ln \frac{d_{o}}{d_{i}}} \text { (toroid) }
$$




$$
\begin{gathered}
\mu^{\prime}-j \mu^{\prime \prime}=\frac{-j\left[\mathcal{Z}_{r}+j \mathcal{Z}_{i}\right]}{\mu_{0} f N^{2} h \ln \frac{d_{o}}{d_{i}}} \text { (toroid) } \\
\mu^{\prime}=\frac{\mathcal{Z}_{i}}{\mu_{0} f N^{2} h \ln \frac{d_{o}}{d_{i}}} \text { (toroid) } \\
\mu^{\prime \prime}=\frac{\mathcal{Z}_{r}}{\mu_{0} f N^{2} h \ln \frac{d_{o}}{d_{i}}} \text { (toroid) }
\end{gathered}
$$

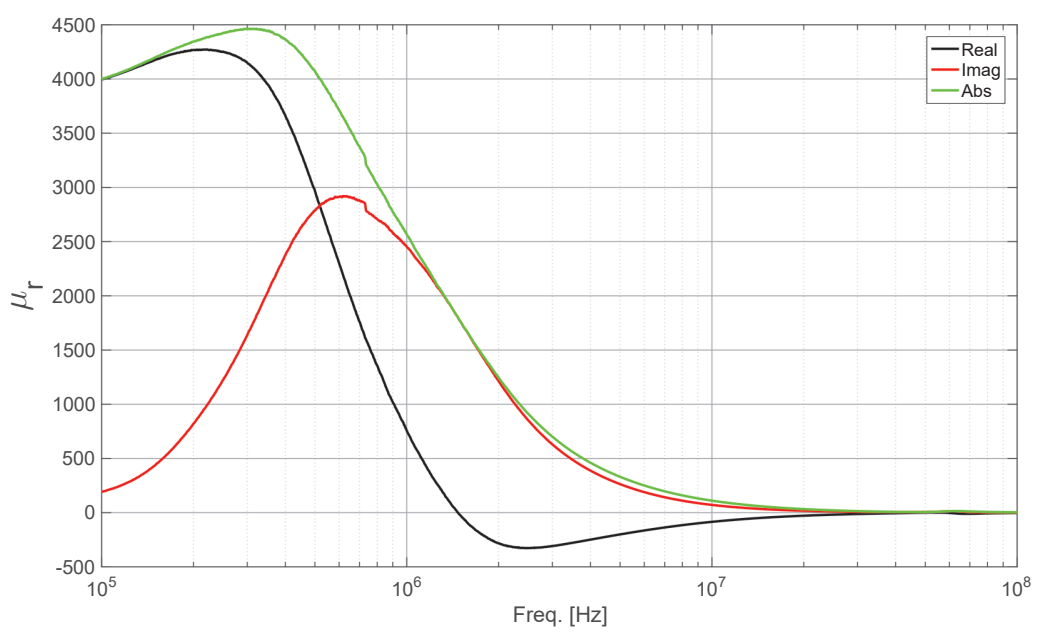

Figure E.3: Result for CMC - WECMBM744823601

In Figure E.3 the result is shown for a typical CMC. Notice real part of $\mu$ crossing zero and becoming negative. From Equation E.2 it can be seen this is related to a negative imaginary part of the impedance, which has a physical representation of a capacitance. It is debatable whether this is a material property or it can be attributed to parasitics of the measurement setup.

\section{E.4 Comparison of methods}

As has been shown, there are multiple ways of acquiring permeability information. In Table E.1 a distinction of four methods have been made, together with their respective availability and accuracy. Figure E.5 shows a comparison between the available permeability extraction methods. In case of the FerroxCube toroidal core, the datasheet indirect method is unavailable as it is a toroidal core and not a full CMC or even an inductor. A large deviation exist between the measured and the datasheet permeability extracted from Figure 4.21a. Acquiring 'correct' permeabilities is very challenging, as the manufacturer already 


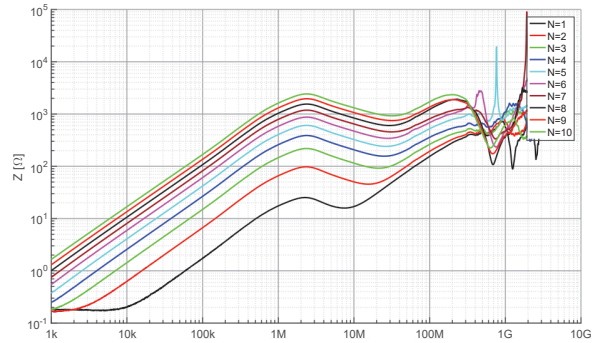

(a) Impedances while increasing windings with equal wire length

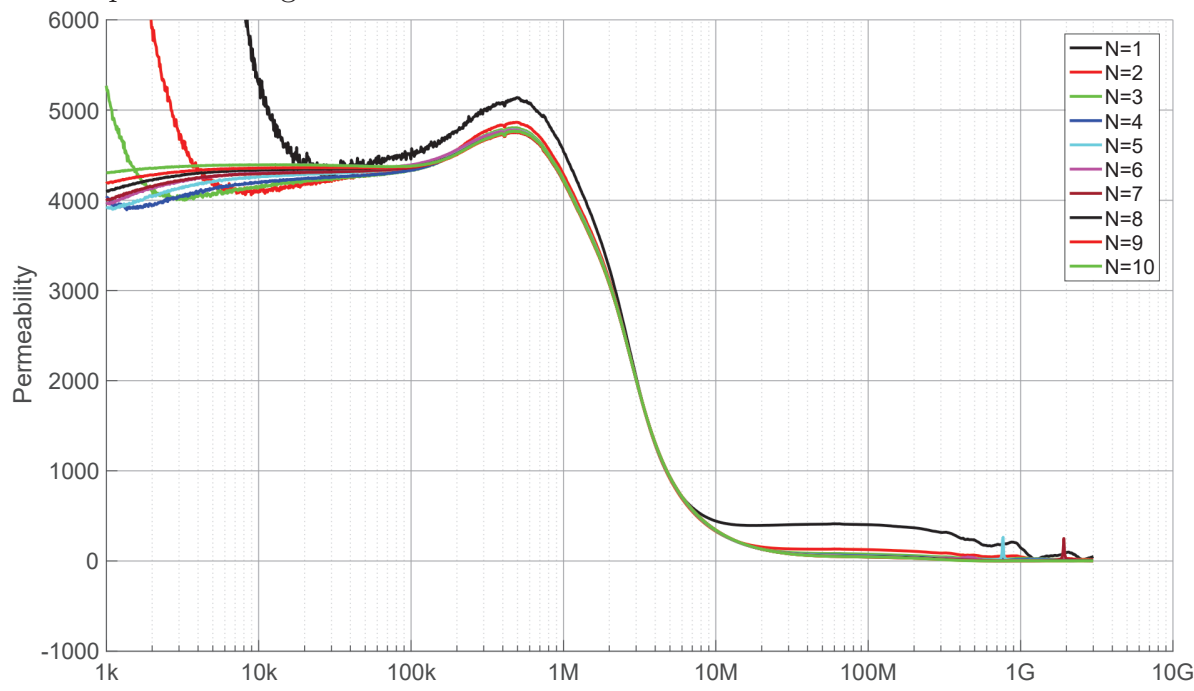

(c) Calculated mu from measured impedances

Figure E.4: Measurements performed of Ferroxcube - TX22/14/6.4-3E27

Table E.1: Permeability Acquisition

\begin{tabular}{|l||c|c|c|}
\hline & Availability & Accuracy & Parasitics Incorporation \\
\hline Datas. Direct & poor & high & unknown \\
\hline Datas. Indirect & good & lowest & yes (unknown) \\
\hline Meas. Core & poor & best & no \\
\hline Meas. Inductor & good & good & yes \\
\hline
\end{tabular}


has deviations between datasheets of the same core material with different dimensions. The datasheet of the 'TX22/14/6.4-3E27' core shows $\mu \approx 5300$, while for the 'TX22/14/13-3E27' $\mu \approx 5000$. While in case of the $3 \mathrm{E} 27$ material datasheet values above 6000 are shown. In these three cases the material itself is identical, but not the dimensions. As the permeability is a material property, it should be independent of the material dimensions.

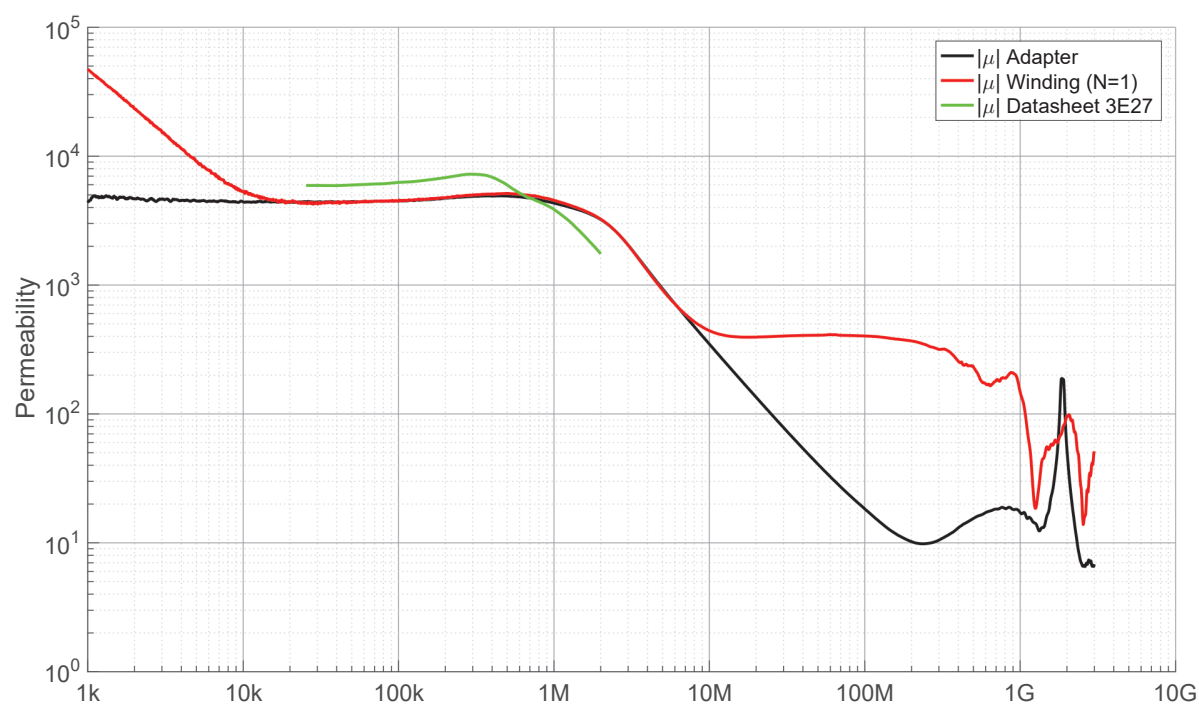

Figure E.5: Comparison of three methods of extracting permeability for FerroxCube $3 \mathrm{E} 27$ material 


\title{
Appendix F
}

\section{Conducted Emission Monitoring}

\begin{abstract}
Measuring large stationary equipment for EMC compliance is difficult, therefore on-site testing is required. Measuring in-situ can be hazardous for people but also for the measurement equipment. Conventional solutions are 1:1000 dividers between the Line-PE and Neutral-PE, which are thus normal-mode measurements only. They are bandwidth limited only by the parasitic behavior of the resistors used. In [95] a new type of monitoring device has been developed. It is able to monitor the mains, with and without a load attached with the conventional 1:1000, but it also includes DM and Normal Mode (Line and Neutral) measurements with a galvanic separated output. A filter has also been included, to suppress the $50 \mathrm{~Hz}$ components and its harmonics, which is useful in case of usage with equipment with low dynamical range. Their outputs are stable over a frequency range of approximately $2 \mathrm{kHz}$ to $2 \mathrm{MHz}$. In the following sections, the box will be shown with instructions how to use it, followed by the measurement results.
\end{abstract}

\section{F.1 The Box}

Figure F.1a shows the IEC C14 plug and the regular EU wall socket, the IEC plug is used as input and thus connected to the either the grid or LISN/AMN. The load can then be connected to the regular EU socket. If required one can always use a conversion adapter for the different loads. In Figure F.1b four BNC type of connectors can be seen with labeling:

- $\mathrm{L}+\mathrm{N} / 1000$

- $\mathrm{DM} / 50$

- $\mathrm{N} / 50$ 
- $\mathrm{L} / 50$

The schematic with component values and names can be seen in Figure F.4. The $\mathrm{L}+\mathrm{N} / 1000$ is the conventional resistive divider that is the top path in the schematic. The resistors are placed in parallel to increase the power that can be handled. Similar design has been applied in the filtered outputs, however a $\mathrm{CMC}$ was used as a 1:1 transformer to provide galvanic separation. Note that this will introduce a phase shift in the output.

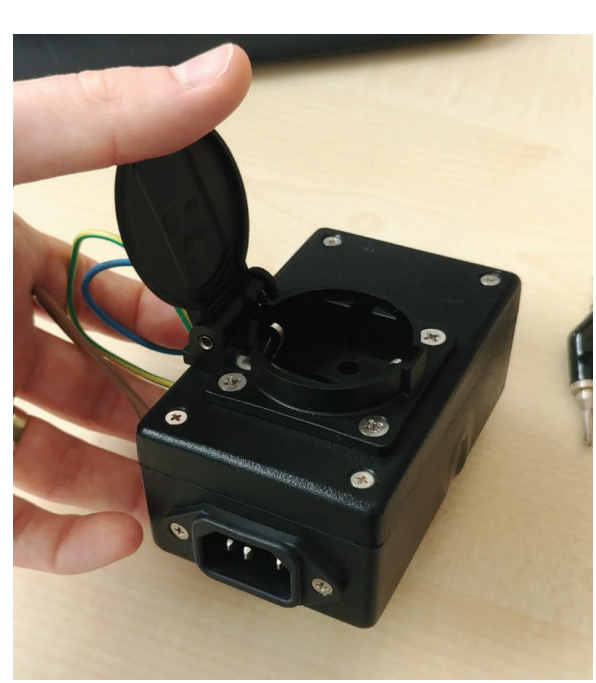

(a) Grid and Load connections

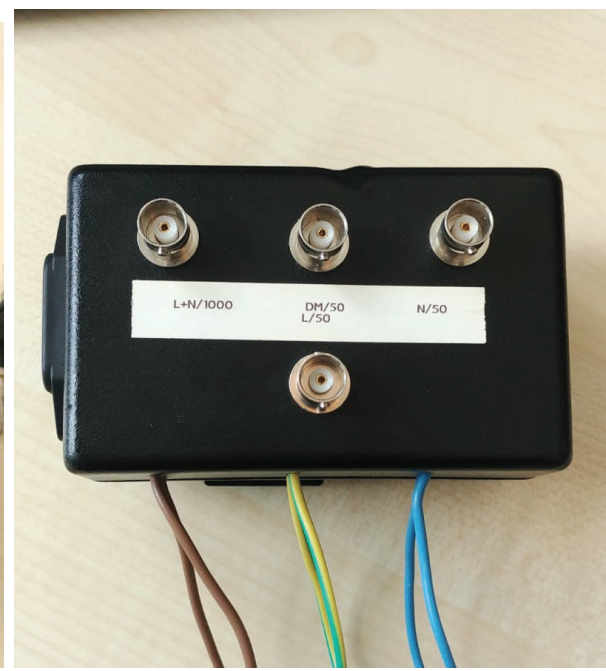

(b) Outputs to measure disturbances

Figure F.1: The conducted disturbances monitoring device that was used in this thesis.

\section{F.2 Measurement}

The mains monitoring device, a VNA measurement has been performed to determine transfer ratios. Fig F.2 shows how the EU power socket to BNC connection was achieved by using a banana to BNC plug. In case of the VNA, two coaxial cable from SMA to BNC where used. The measurement results can be seen in Figure F.3. Expected are attenuations of $34 \mathrm{~dB}$, which is depicted with the dashed black line. Two vertical lines are added to indicate the frequency range of interest for which this device was developed. This particular box has also been used in $[34,96]$. 


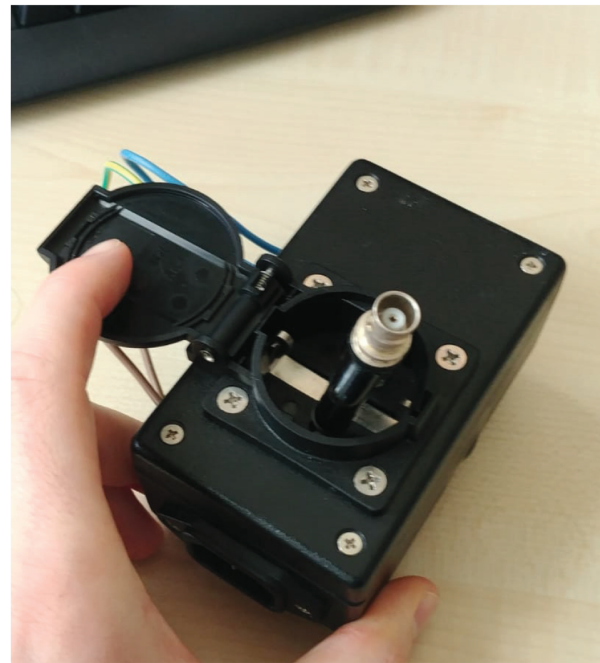

Figure F.2: How to connect to a VNA/EMI receiver with tracking generator.

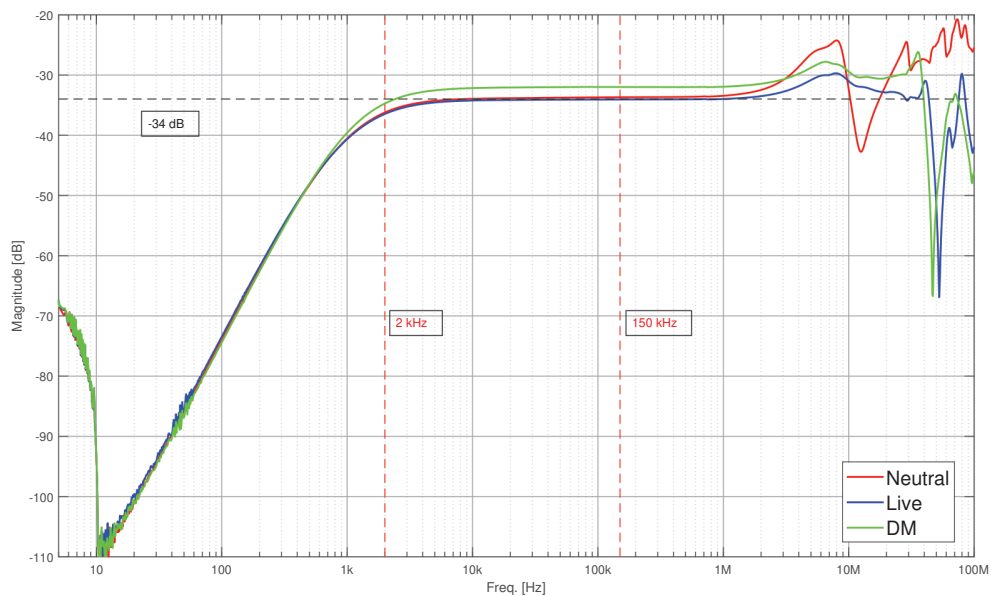

Figure F.3: Voltage transfer ratios of mains monitoring device.

\section{F.3 Usage}

The filtered outputs require a $50 \Omega$ termination. So either use an external one, or switch it internally in the scope. The resistive divider does not require this termination. 


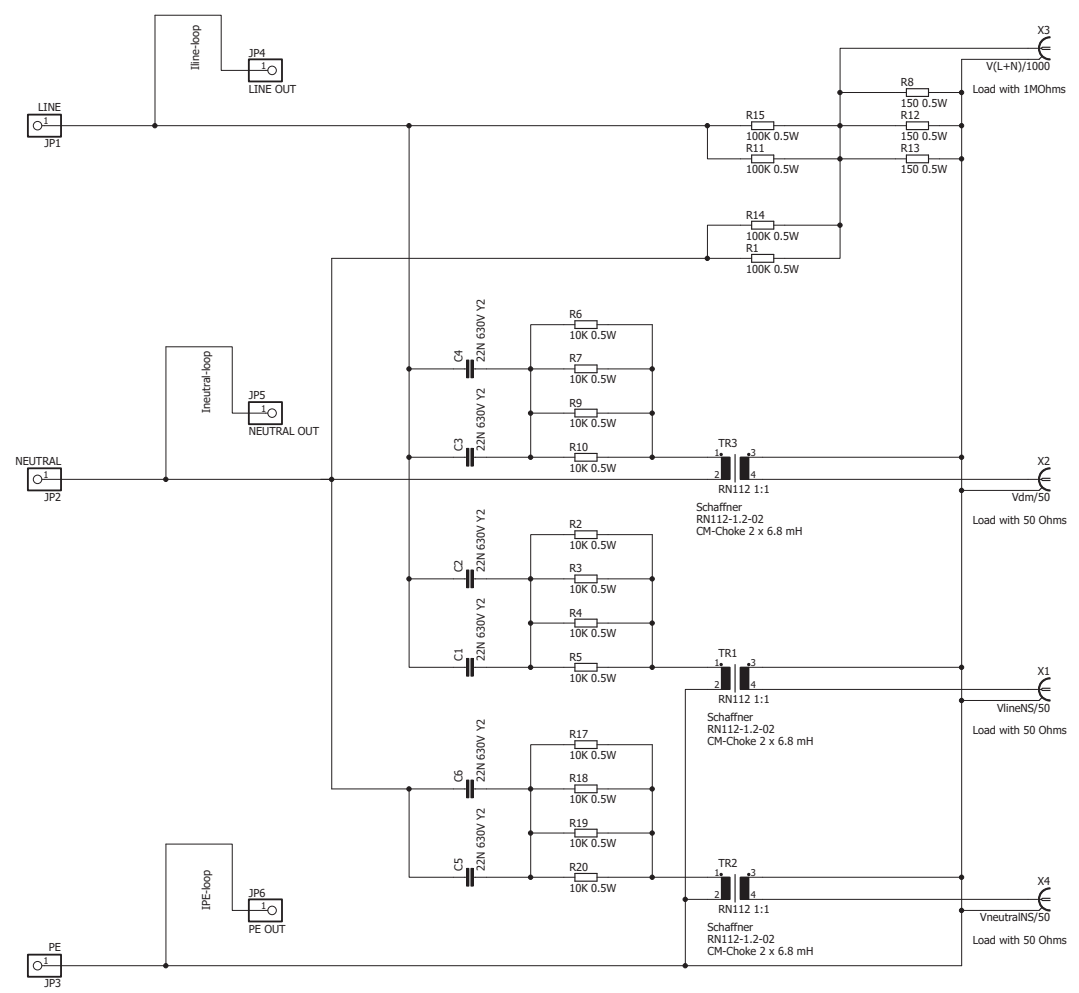

Figure F.4: The inside schematic of the monitoring devices as was provided by Frits Buesink. 